\title{
Search for the Standard Model Higgs Boson in $H \rightarrow W W \rightarrow \ell \nu j j$ Channel in 1.96-TeV Proton-Antiproton Collisions
}

\author{
Yuji Sudo
}

February 2012 



\title{
Search for the Standard Model Higgs Boson in $H \rightarrow W W \rightarrow \ell \nu j j$ Channel in 1.96-TeV Proton-Antiproton Collisions
}

\author{
Yuji Sudo \\ (Doctoral Program in Physics)
}

Submitted to the Graduate School of

Pure and Applied Sciences

in Partial Fulfillment of the Requirements for the Degree of Doctor of Philosophy in

Science

at the

University of Tsukuba 



\begin{abstract}
We searched for Standard Model Higgs boson decaying into $W W^{(*)}$, where one of the $W$ bosons decays leptonically and the other hadronically. We used $4.6 \mathrm{fb}^{-1}$ of data collected with the CDF detector in Tevatron Run II. We composed a likelihood discriminant using kinematic variables in order to maximize the signal/background separation. We set upper limits on Higgs boson production cross section for Higgs boson masses between 150 and $200 \mathrm{GeV} / c^{2}$. The results range from $5.69\left(m_{H}=170 \mathrm{GeV} / c^{2}\right)$ to $52.5\left(m_{H}=150 \mathrm{GeV} / c^{2}\right)$ times the Standard Model values at $95 \%$ confidence level.
\end{abstract}




\section{Acknowledgments}

First of all, I would like to express my appreciation to everyone who supported me during the Ph.D program. I would like to express my sincere gratitude to Prof. Fumihiko Ukegawa and Prof. Shinhong Kim. They gave me a chance to study at Fermilab. It is meaningful experience for me. I would like to thank Prof. Koji Sato very much. He strongly supported my analysis. I also would like to thank HDG group conveners, Dr. Eric B. James, Prof. Robert Craig Group, Dr. Homer F Wolfe. They gave me very usefull comments to progress this study. I would like to express my appreciation to Prof. Kazuhiko Hara and Prof. Yuji Takeuchi. They always gave me constructive advice. I would like to thank HEP Tsukuba members, Dr. Hideki Miyake, Dr. Masakazu Kurata, Kenichi Takemasa. I would like to thank CDF Japan group members, Prof. Kohei Yorita, Dr. Tomonobu Tomura, Dr. Koji Ebina, Dr. Junji Naganoma, Dr. Naoki Kimura, Atsunari Hamaguchi, Daisuke Yamato and Yuki Sakurai. And I also would like to thank Dr. Masato Aoki and Dr. Yoshikazu Nagai. They gave me many usefull advice. I would like to thank Prof. Satoru Uozumi. He encourage me at FermiLab. I would like to thank secretaries very much, Mrs. Kazuko Kumasiro and Mrs. Kyoko Kunori. They helped me many times. At last I would like to my sincere gratitude to my parents. They have always supported me very much. 


\section{Contents}

1 Introduction 1

1.1 The Standard Model . . . . . . . . . . . . . . . . . . . . . . . 1

1.1 .1 Fermions . . . . . . . . . . . . . . . . . 1

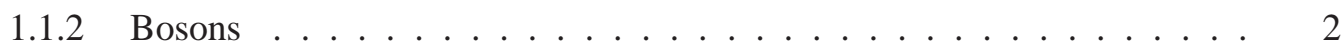

1.1 .3 QCD Lagrangian . . . . . . . . . . . . . . . . . 3

1.1.4 Electroweak Interactions . . . . . . . . . . . . . . . . 3

1.1.5 Fermion Mass Term . . . . . . . . . . . . . . . 4

1.1.6 Mass Term of $W$ and $Z \ldots \ldots \ldots \ldots \ldots$

1.1 .7 Higgs Interaction $\ldots \ldots \ldots \ldots \ldots \ldots$

1.1.8 Higgs Boson Production and Decay . . . . . . . . . . . . . . 5

1.2 Previous Higgs Boson Searches _ . . . . . . . . . . . . . . . . 8

1.2.1 Direct Searches . . . . . . . . . . . . . . . . . . . 8

1.2 .2 Indirect Searches . . . . . . . . . . . . . . . . . . . 11

2 Experimental Apparatus $\quad 13$

2.1 Characteristics of Fermilab Accelerators . . . . . . . . . . . . . . . . . 13

2.2 The CDF II Detector . . . . . . . . . . . . . . . . . . . . 16

2.2.1 Tracking System . . . . . . . . . . . . . . . . . . 17

2.2 .2 Calorimeters .......................... 20

2.2 .3 Muon Detectors . . . . . . . . . . . . . . . . . 22

2.2 .4 Luminosity Monitor . . . . . . . . . . . . . . . . . . . 24

2.2.5 Data Acquisition System . . . . . . . . . . . . . . 25

3 Event Reconstruction 29

3.1 High Level Detector Object . . . . . . . . . . . . . . . . . . . . . . . . . . . . . 29

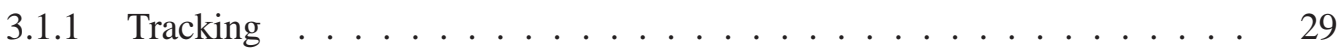

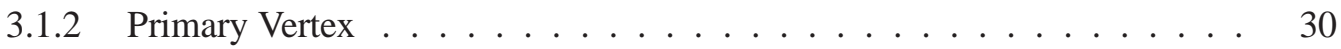

3.1.3 Calorimeter Clustering . . . . . . . . . . . . . . . 31

3.2 Particle Identification $\ldots \ldots \ldots \ldots \ldots \ldots \ldots$

3.2 .1 Leptons . . . . . . . . . . . . . . . . . . . . . . . 31

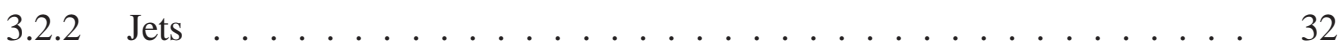

3.2.3 Missing Transverse Energy . . . . . . . . . . . . . . . . . . 33

3.2 .4 Neutrino $p_{z}^{\nu}$ Reconstruction $\ldots \ldots \ldots \ldots \ldots . \ldots \ldots$

3.2.5 Distinction of Up- and Down-type Quark Jets . . . . . . . . . . . . . . 37 
4 Event Selection $\quad 39$

4.1 Trigger Requirements . . . . . . . . . . . . . . . . . . . . . . . . . . . . 39

$4.1 .1 \quad$ ELECTRON_CENTRAL_18 . . . . . . . . . . . . . . . 39

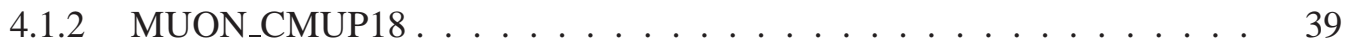

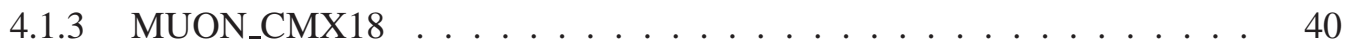

4.2 Event Selection . . . . . . . . . . . . . . . . . . . . . . 40

5 Background and Signal Estimation 43

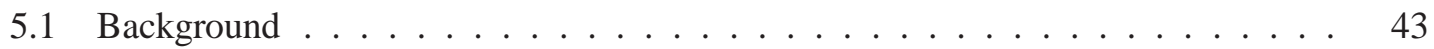

5.2 Likelihood Composition . . . . . . . . . . . . . . . . 43

5.3 Method and the Result of Background and Signal Estimation . . . . . . . . . . 46

6 Systematic Uncertainties $\quad 55$

6.1 Rate Uncertainties . . . . . . . . . . . . . . . . . . . . . . . . . 55

6.1 .1 Luminosity . . . . . . . . . . . . . . . . . 55

6.1.2 Trigger Efficiency and Lepton Reconstruction Efficiency . . . . . . . . . 55

6.1 .3 Signal Cross Section . . . . . . . . . . . . . . . . . 55

6.1 .4 Jet Energy Scale Uncertainty . . . . . . . . . . . . . . . . . . . . . . . 56

6.1 .5 non- $W$ normalization . . . . . . . . . . . . . . 56

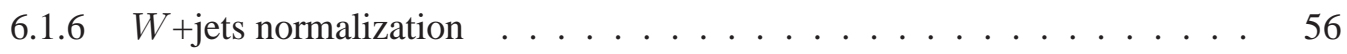

$6.1 .7 Z+$ jets normalization $\ldots \ldots \ldots \ldots \ldots \ldots$

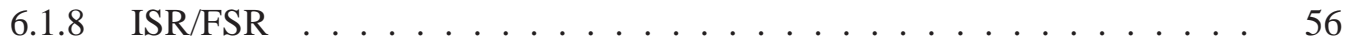

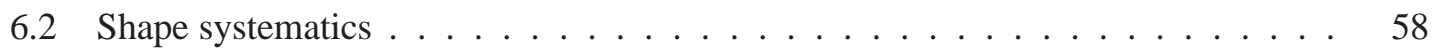

6.2 .1 Jet energy scale in signal . . . . . . . . . . . . . . . . 58

6.2 .2 Jet energy scale in $W+$ jets background . . . . . . . . . . . . 58

$6.2 .3 Q^{2}$ of $W+$ jets background $\ldots \ldots \ldots \ldots \ldots$

7 Results and Discussions $\quad 63$

7.1 Upper Limits on Higgs Boson Production Cross Section . . . . . . . . . . . . 63

7.2 Discussion . . . . . . . . . . . . . . . . . 63

8 Conclusions $\quad 67$

$\begin{array}{lr}\text { A Signal and Background Templates } & 69\end{array}$

B Input Variables and Output of the Likelihood $\quad 81$

$\begin{array}{lll}\text { C } & \mathbf{Q}^{2} \text { and JES Shape Systematics } & 89\end{array}$ 


\section{List of Figures}

1.1 The NLO Standard Model Higgs boson production cross section in $\mathrm{pb}$ at the Tevatron with $p \bar{p}$ collision at $\sqrt{s}=1.96 \mathrm{TeV} \ldots \ldots \ldots \ldots \ldots$

1.2 The branching ratio for each Higgs boson decay mode as a function of Higgs

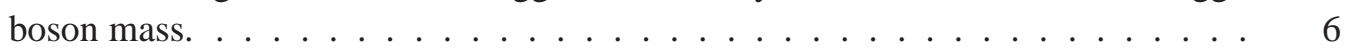

1.3 Feynman diagram of the $g g \rightarrow H \rightarrow W W \rightarrow \ell \nu \ell \nu$ channel. . . . . . . . . 7

1.4 Feynman diagram of the $g g \rightarrow H \rightarrow W W \rightarrow \ell \nu j j$ channel. . . . . . . . . 7

1.5 The ratio $C L_{s}=C L_{s+b} / C L_{b}$ for the signal plus background hypothesis as a function of the Higgs mass . . . . . . . . . . . . . . 8

1.6 The summary of the CDF combined $95 \%$ upper limit on the Higgs boson production cross section . . . . . . . . . . . . . . . . . . 10

1.7 The summary of the Tevatron combined the Higgs boson search . . . . . . . . . 10

1.8 The $\Delta \chi^{2}$ distribution as a function of the Higgs mass . . . . . . . . . . . . . 12

2.1 A diagram of the Fermilab accelerator chain. . . . . . . . . . . . . . . . . 14

2.2 Isometric view of the CDF II detector. . . . . . . . . . . . . . . . 17

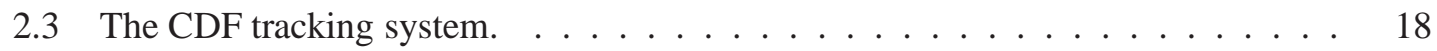

2.4 The CDF silicon detector. Left: the coverage of the silicon detector in r-z plane. Right: Configuration of the silicon detector in $\mathrm{r}-\phi$ plane. . . . . . . . . . . . . 19

2.5 The CDF calorimeter system. . . . . . . . . . . . . . . . . 20

2.6 The single CEM wedge. . . . . . . . . . . . . . . . . 21

2.7 CDF muon coverage for the CMU, CMP, CMX, and BMU detectors. The BMU is referred as the IMU. . . . . . . . . . . . . . . . . . 23

2.8 A cross section view of a CMU muon chamber. . . . . . . . . . . . . . 23

2.9 Total integrated luminosity delivered by the Tevatron and recorded by the CDF. . 24

2.10 Data flow in the CDF data acquisition system. . . . . . . . . . . . 25

2.11 Block diagram of the Level 1 and Level 2 trigger path. . . . . . . . . . . . 26

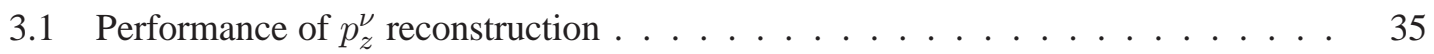

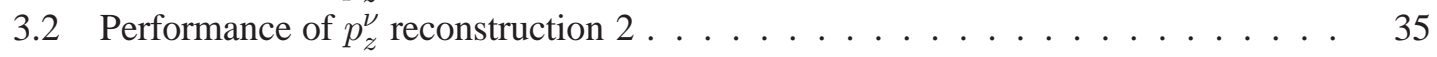

3.3 Fit of the reconstructed Higgs mass to a Gaussian . . . . . . . . . . . . 36

$3.4 \cos \theta_{l}^{*}$ against $\cos \theta_{u p-t y p e}^{*}$. From left: signal $\mathrm{MC}$ for $m_{H}=170 \mathrm{GeV} / \mathrm{c}^{2}$, electroweak $W W$ process, and $W+c+1$ parton. . . . . . . . . . . . . 37

3.5 Blue(Red) histogram shows $\Delta \phi$ distribution between lepton and the up-type(downtype) jet in the Higgs decay. For signal Monte Carlo for $m_{H}=170 \mathrm{GeV} / \mathrm{c}^{2}$. . . 37

5.1 Likelihood templates for the CEM at $m_{H}=180 \mathrm{GeV} / \mathrm{c}^{2} \ldots \ldots \ldots$. . . . . 44

5.2 Likelihood templates for the CMUP at $m_{H}=180 \mathrm{GeV} / \mathrm{c}^{2} \ldots \ldots \ldots$. . . . . 45

5.3 Likelihood templates for the CMX at $m_{H}=180 \mathrm{GeV} / \mathrm{c}^{2} \ldots \ldots \ldots \ldots$

$5.4 E_{T}$ fit result for $m_{H}=180 \mathrm{GeV} / c^{2} \ldots \ldots \ldots \ldots \ldots \ldots$ 
5.5 Fit to log likelihood distribution in the final iteration, for an assumed Higgs boson mass of $180 \mathrm{GeV} / \mathrm{c}^{2}$. Red line shows the signal distribution with SM cross section times a hundred. . . . . . . . . . . . . . . . . 48

5.6 Likelihood input distributions for $m_{H}=180 \mathrm{GeV} / \mathrm{c}^{2} \ldots \ldots \ldots \ldots 2$

5.7 Likelihood output distribution for $m_{H}=180 \mathrm{GeV} / \mathrm{c}^{2} \ldots \ldots \ldots$. . . . . 53

6.1 Likelihood output for the $W+$ jets quark JES $+1 \sigma$ systematic samples at $m_{H}=$

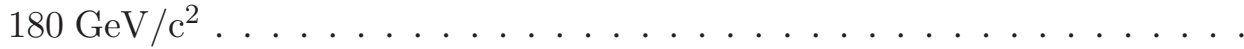

6.2 Likelihood output for the $W+$ jets quark JES $\pm 1 \sigma$ systematic samples at $m_{H}=$

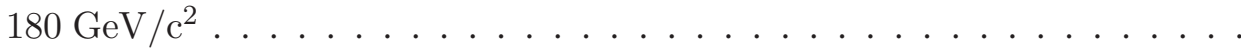

6.3 Likelihood output for the $W+$ jets gluon JES $+1 \sigma$ systematic samples at $m_{H}=$

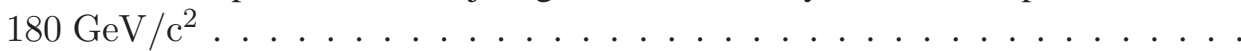

6.4 Likelihood output for the $W+$ jets gluon JES $\pm 1 \sigma$ systematic samples at $m_{H}=$

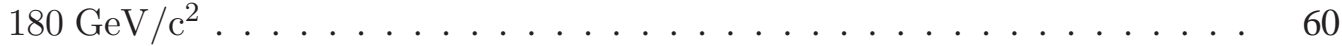

6.5 Likelihood output for the $W+$ jets $\mathrm{Q}^{2}$ systematic samples at $m_{H}=180 \mathrm{GeV} / \mathrm{c}^{2}$. $\quad 60$

6.6 Likelihood output for the $W+$ jets $\mathrm{Q}^{2}$ systematic samples at $m_{H}=180 \mathrm{GeV} / \mathrm{c}^{2}$. $\quad 61$

7.1 The dashed line and the solid line show the expected and observed upper limits on cross section normalized by SM Higgs boson cross section. . . . . . . . . . . . 64

7.2 Result of $H \rightarrow \ell \nu j j$ search at ATLAS experiment. . . . . . . . . . . 65

A.1 Likelihood templates for the CEM, $m_{H}=150 \mathrm{GeV} / \mathrm{c}^{2} \ldots \ldots \ldots$

A.2 Likelihood templates for the CMUP, $m_{H}=150 \mathrm{GeV} / \mathrm{c}^{2} \ldots \ldots \ldots \ldots$

A.3 Likelihood templates for the CMX, $m_{H}=150 \mathrm{GeV} / \mathrm{c}^{2} \ldots \ldots \ldots \ldots$

A.4 Likelihood templates for the CEM, $m_{H}=160 \mathrm{GeV} / \mathrm{c}^{2} \ldots \ldots \ldots \ldots$

A.5 Likelihood templates for the CMUP, $m_{H}=160 \mathrm{GeV} / \mathrm{c}^{2} \ldots \ldots \ldots \ldots$

A.6 Likelihood templates for the CMX, $m_{H}=160 \mathrm{GeV} / \mathrm{c}^{2} \ldots \ldots \ldots \ldots$

A.7 Likelihood templates for the CEM, $m_{H}=170 \mathrm{GeV} / \mathrm{c}^{2} \ldots \ldots \ldots \ldots$

A.8 Likelihood templates for the CMUP, $m_{H}=170 \mathrm{GeV} / \mathrm{c}^{2} \ldots \ldots \ldots \ldots$. . . . . . . . . . .

A.9 Likelihood templates for the CMX, $m_{H}=170 \mathrm{GeV} / \mathrm{c}^{2} \ldots \ldots \ldots \ldots$

A.10 Likelihood templates for the CEM, $m_{H}=180 \mathrm{GeV} / \mathrm{c}^{2} \ldots \ldots \ldots \ldots$

A.11 Likelihood templates for the CMUP, $m_{H}=180 \mathrm{GeV} / \mathrm{c}^{2} \ldots \ldots \ldots \ldots$

A.12 Likelihood templates for the CMX, $m_{H}=180 \mathrm{GeV} / \mathrm{c}^{2} \ldots \ldots \ldots$

A.13 Likelihood templates for the CEM, $m_{H}=190 \mathrm{GeV} / \mathrm{c}^{2} \ldots \ldots \ldots$. . . . . 77

A.14 Likelihood templates for the CMUP, $m_{H}=190 \mathrm{GeV} / \mathrm{c}^{2} \ldots \ldots \ldots \ldots$

A.15 Likelihood templates for the CMX, $m_{H}=190 \mathrm{GeV} / \mathrm{c}^{2} \ldots \ldots \ldots$

A.16 Likelihood templates for the CEM, $m_{H}=200 \mathrm{GeV} / \mathrm{c}^{2} \ldots \ldots \ldots$. . . . . . 79

A.17 Likelihood templates for the CMUP, $m_{H}=200 \mathrm{GeV} / \mathrm{c}^{2} \ldots \ldots \ldots$. . . . . 80

A.18 Likelihood templates for the CMX, $m_{H}=200 \mathrm{GeV} / \mathrm{c}^{2} \ldots \ldots \ldots$

B.1 Likelihood input distributions for $m_{H}=150 \mathrm{GeV} / \mathrm{c}^{2} \ldots \ldots \ldots$. . . . . 81

B.2 Likelihood output distribution for $m_{H}=150 \mathrm{GeV} / \mathrm{c}^{2} \ldots \ldots \ldots \ldots$. . . . . . . 82

B.3 Likelihood input distributions for $m_{H}=160 \mathrm{GeV} / \mathrm{c}^{2} \ldots \ldots \ldots$. . . . . . 83

B.4 Likelihood output distribution for $m_{H}=160 \mathrm{GeV} / \mathrm{c}^{2} \ldots \ldots \ldots \ldots$

B.5 Likelihood input distributions for $m_{H}=170 \mathrm{GeV} / \mathrm{c}^{2} \ldots \ldots \ldots \ldots$. . . . . 84

B.6 Likelihood output for the analysis at $m_{H}=170 \mathrm{GeV} / \mathrm{c}^{2} \ldots \ldots \ldots$. . . . . 84

B.7 Likelihood input distributions for $m_{H}=180 \mathrm{GeV} / \mathrm{c}^{2} \ldots \ldots \ldots \ldots$

B.8 Likelihood output distribution for $m_{H}=180 \mathrm{GeV} / \mathrm{c}^{2} \ldots \ldots \ldots \ldots$

B.9 Likelihood input distributions for $m_{H}=190 \mathrm{GeV} / \mathrm{c}^{2} \ldots \ldots \ldots$. . . . . 86 
B.10 Likelihood output distribution for $m_{H}=190 \mathrm{GeV} / \mathrm{c}^{2} \ldots \ldots \ldots$. . . . 86

B.11 Likelihood input distributions for $m_{H}=200 \mathrm{GeV} / \mathrm{c}^{2} \ldots \ldots \ldots \ldots$. . . . . . . . . . 87

B.12 Likelihood output distribution for $m_{H}=200 \mathrm{GeV} / \mathrm{c}^{2} \ldots \ldots \ldots$. . . . 87

C.1 Likelihood output distribution using the $W+$ jets $\mathrm{Q}^{2}$ systematic samples for $m_{H}=$

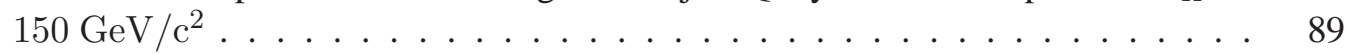

C.2 Likelihood output distribution using the $W$ +jets $Q^{2}$ systematic samples $\ldots \ldots$. . 90

C.3 Likelihood output distribution using the $W+$ jets $\mathrm{Q}^{2}$ systematic samples for $m_{H}=$

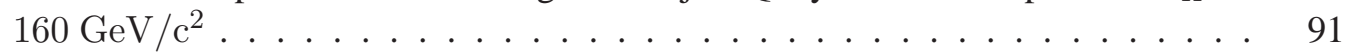

C.4 Likelihood output distribution using the $W+$ jets $Q^{2}$ systematic samples $\ldots \ldots$. . . 92

C.5 Likelihood output distribution using the $W+$ jets $\mathrm{Q}^{2}$ systematic samples for $m_{H}=$ $170 \mathrm{GeV} / \mathrm{c}^{2} \ldots \ldots \ldots \ldots \ldots \ldots \ldots$

C.6 Likelihood output distribution using the $W+$ jets $Q^{2}$ systematic samples $\ldots \ldots$. . 94

C.7 Likelihood output distribution using the $W+$ jets $\mathrm{Q}^{2}$ systematic samples for $m_{H}=$ $180 \mathrm{GeV} / \mathrm{c}^{2} \ldots \ldots \ldots \ldots \ldots \ldots$

C.8 Likelihood output distribution using the $W+$ jets $Q^{2}$ systematic samples $\ldots \ldots$. . 96

C.9 Likelihood output distribution using the $W+$ jets $\mathrm{Q}^{2}$ systematic samples for $m_{H}=$

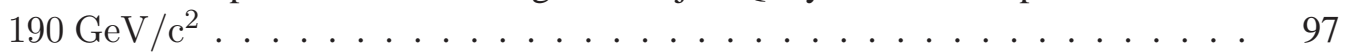

C.10 Likelihood output distribution using the $W+$ jets $Q^{2}$ systematic samples $\quad \ldots \ldots$. . 98

C.11 Likelihood output distribution using the $W+$ jets $\mathrm{Q}^{2}$ systematic samples for $m_{H}=$

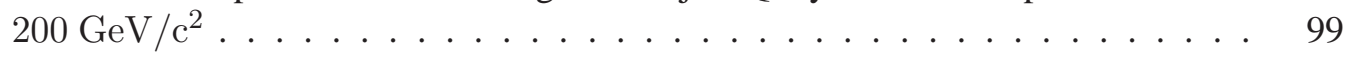

C.12 Likelihood output distribution using the $W$ +jets $Q^{2}$ systematic samples . . . . . 100

C.13 Likelihood output distribution using the $W+$ jets quark JES $+1 \sigma$ systematic samples for $m_{H}=150 \mathrm{GeV} / \mathrm{c}^{2} \ldots \ldots \ldots \ldots \ldots$. . . . . . . . . . 101

C.14 Likelihood output distribution using the $W+$ jets quark JES $\pm 1 \sigma$ systematic samples 102

C.15 Likelihood output distribution using the $W+$ jets gluon JES $+1 \sigma$ systematic samples for $m_{H}=150 \mathrm{GeV} / \mathrm{c}^{2} \ldots \ldots \ldots \ldots \ldots$. . . . . . . . . . 102

C.16 Likelihood output distribution using the $W+$ jets gluon JES $\pm 1 \sigma$ systematic samples 103

C.17 Likelihood output distribution using the $W+$ jets quark JES $+1 \sigma$ systematic samples for $m_{H}=160 \mathrm{GeV} / \mathrm{c}^{2} \ldots \ldots \ldots \ldots$. . . . . . . . . . . 104

C.18 Likelihood output distribution using the $W+$ jets quark JES $\pm 1 \sigma$ systematic samples 105

C.19 Likelihood output distribution using the $W+$ jets gluon JES $+1 \sigma$ systematic samples for $m_{H}=160 \mathrm{GeV} / \mathrm{c}^{2} \ldots \ldots \ldots \ldots \ldots$. . . . . . . . . . 105

C.20 Likelihood output distribution using the $W+$ jets gluon JES $\pm 1 \sigma$ systematic samples 106

C.21 Likelihood output distribution using the $W+$ jets quark JES $+1 \sigma$ systematic samples for $m_{H}=170 \mathrm{GeV} / \mathrm{c}^{2} \ldots \ldots \ldots \ldots$. . . . . . . . . 107

C.22 Likelihood output distribution using the $W+$ jets quark JES $\pm 1 \sigma$ systematic samples 108

C.23 Likelihood output distribution using the $W+$ jets gluon JES $+1 \sigma$ systematic samples for $m_{H}=170 \mathrm{GeV} / \mathrm{c}^{2} \ldots \ldots \ldots \ldots \ldots$. . . . . . . . . . 108

C.24 Likelihood output distribution using the $W+$ jets gluon JES $\pm 1 \sigma$ systematic samples 109

C.25 Likelihood output distribution using the $W+$ jets quark JES $+1 \sigma$ systematic samples for $m_{H}=180 \mathrm{GeV} / \mathrm{c}^{2} \ldots \ldots \ldots \ldots \ldots \ldots$. . . . . . . . . . 110

C.26 Likelihood output distribution using the $W+$ jets quark JES $\pm 1 \sigma$ systematic samples 111

C.27 Likelihood output distribution using the $W+$ jets gluon JES $+1 \sigma$ systematic sam-

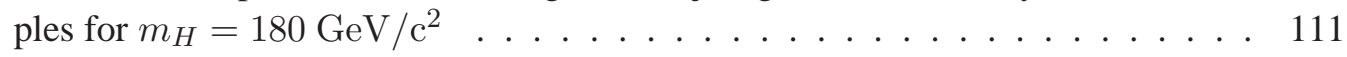

C.28 Likelihood output distribution using the $W+$ jets gluon JES $\pm 1 \sigma$ systematic samples 112

C.29 Likelihood output distribution using the $W+$ jets quark JES $+1 \sigma$ systematic samples for $m_{H}=190 \mathrm{GeV} / \mathrm{c}^{2} \ldots \ldots \ldots \ldots \ldots \ldots \ldots$ 
C.30 Likelihood output distribution using the $W+$ jets quark JES $\pm 1 \sigma$ systematic samples 114

C.31 Likelihood output distribution using the $W+$ jets gluon JES $+1 \sigma$ systematic samples for $m_{H}=190 \mathrm{GeV} / \mathrm{c}^{2} \ldots \ldots \ldots \ldots \ldots \ldots$. . . . . . . . . . 114

C.32 Likelihood output distribution using the $W+$ jets gluon JES $\pm 1 \sigma$ systematic samples 115

C.33 Likelihood output distribution using the $W+$ jets quark JES $+1 \sigma$ systematic samples for $m_{H}=200 \mathrm{GeV} / \mathrm{c}^{2} \ldots \ldots \ldots \ldots \ldots \ldots$

C.34 Likelihood output distribution using the $W+$ jets quark JES $\pm 1 \sigma$ systematic samples 117

C.35 Likelihood output distribution using the $W+$ jets gluon JES $+1 \sigma$ systematic samples for $m_{H}=200 \mathrm{GeV} / \mathrm{c}^{2} \ldots \ldots \ldots \ldots \ldots$. . . . . . . . . . 117

C.36 Likelihood output distribution using the $W+$ jets gluon JES $\pm 1 \sigma$ systematic samples 118 


\section{List of Tables}

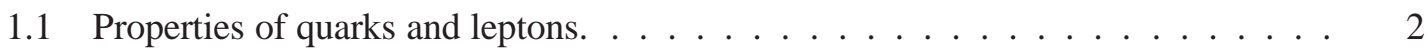

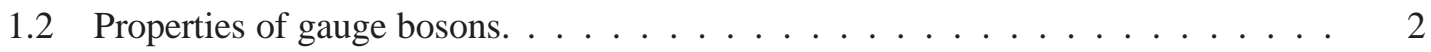

3.1 Selection requirement for central electrons [31] . . . . . . . . . . . . . . . 32

3.2 Selection requirement for central muons [31]. . . . . . . . . . . . . . 33

3.3 Fraction of events with imaginary solutions, and of events where we pick up the correct neutrino $P_{\mathrm{Z}}$ solution. The resolution and mass for the reconstructed Higgs

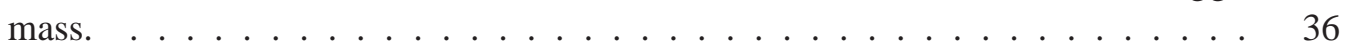

5.1 Cross sections of the electroweak background processes. . . . . . . . . . . 46

5.2 Cross sections for the signal events, generated with PYTHIA parameters PARP(91)

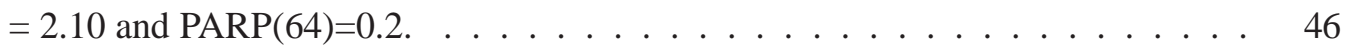

5.3 Constraints for each background and signal fluctuation in the iteration process. . 47

5.4 Estimates of the background events by the iteration. . . . . . . . . . . . . . 49

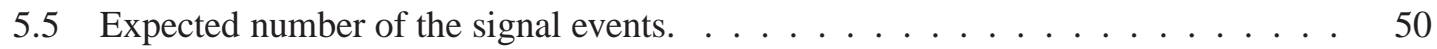

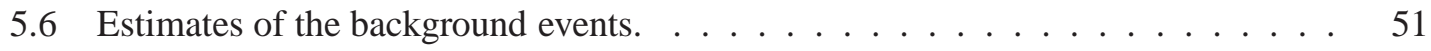

6.1 elative systematic uncertainties on the event rates. . . . . . . . . . . 57

7.1 Expected and observed 95\% C.L. upper limits on cross section for the Standard Model higgs boson in $H \rightarrow W W^{(*)} \rightarrow l \nu j j$ channel. The values are normalized by the Standard Model Higgs boson cross sections. . . . . . . . . . . . . . . 64 



\section{Chapter 1}

\section{Introduction}

Searches for the Higgs boson are one of the most interesting subjects of the Tevatron Run II program. For searches for a high mass Higgs boson, $g g \rightarrow H \rightarrow W W^{(*)}$ has the largest production rate and is considered as the most promising search channel. The dilepton final state, where both W bosons decay leptonically $\left(g g \rightarrow H \rightarrow W W^{(*)} \rightarrow l \nu l \nu\right.$ ), gives a clean search sample and we already have a state of art analysis on this channel [1].

In this thesis, we explore the semi-leptonic final state where one of the $W$ 's decays leptonically while the other decays hadronically $\left(g g \rightarrow H \rightarrow W W^{(*)} \rightarrow l \nu j j\right)$. This semi-leptonic channel has a branching fraction about six times larger than the dilepton channel. On the other hand, it suffers from a large background of $\mathrm{W}$ production associated with QCD jets.

We compose a likelihood discriminant from several kinematic variables in order to maximize our signal/background separation.

In this study, we use $4.6 \mathrm{fb}^{-1}$ of data collected from February 2002 through May 2009.

\subsection{The Standard Model}

The Standard Model is a quantum field theory that is composed of $S U(3)$ and $S U(2) \times U(1)$ gauge symmetry groups.

Gauge theory describes particle interaction. Electroweak interaction is the unified interaction of the Electromagnetic and weak interactions. $S U(2) \times U(1)$ gauge symmetry describes the electroweak interaction. The electromagnetic interaction satisfies strictly the gauge symmetry. Then photon is a massless particle. The strong interaction is generated by color charge which is quantum number related to quarks. the strong interaction satisfies strictly the $S U(3)$ gauge symmetry (QCD : quantum chromodynamics $[2,3])$.

The Standard Model take a stand on that fundamental particle mass is essentially zero. The chiral invariance gives a theoretical base to that fermion, which constitute matter, is massless particle. On the other hand, gauge invariance gives theoretical base to that gauge boson mass is zero. These symmetries are broken spontaneously, then fandamental particle mass is generated. It is named higgs mechanismm $[4,5]$.

\subsubsection{Fermions}

Fermions have spin $s=\frac{1}{2}$ and are classified to quarks and leptons which are considered the fundamental particles what is the world made of. These fermions are classified into the three generations of left-handed and right-handed quarks and leptons. The left-handed fermions are in weak isospin doublets. On the other hand, the right-handed fermions are in weak isospin singlets. 
Table 1.1: Properties of quarks and leptons.

\begin{tabular}{c|c|c|c|c}
\hline \hline & Particle & Weak isospin $I_{3}$ & Electric charge $Q$ & Hyper charge $Y$ \\
\hline \multirow{4}{*}{ Quarks } & $u_{L}, c_{L}, t_{L}$ & $1 / 2$ & $2 / 3$ & $1 / 3$ \\
& $d_{L}, s_{L}, b_{L}$ & $-1 / 2$ & $-1 / 3$ & $1 / 3$ \\
\cline { 2 - 5 } & $u_{R}, c_{R}, t_{R}$ & 0 & $2 / 3$ & $4 / 3$ \\
& $d_{R}, s_{R}, b_{R}$ & 0 & $-1 / 3$ & $-2 / 3$ \\
\hline \multirow{3}{*}{ Leptons } & $\nu_{e}, \nu_{\mu}, \nu_{\tau}$ & $1 / 2$ & 0 & -1 \\
& $e_{L}, \mu_{L}, \tau_{L}$ & $-1 / 2$ & -1 & -1 \\
\cline { 2 - 5 } & $e_{R}, \mu_{R}, \tau_{R}$ & 0 & -1 & -2 \\
\hline \hline
\end{tabular}

Table 1.2: Properties of gauge bosons.

\begin{tabular}{c|c|c|c|c}
\hline \hline Particle & Spin $s$ & Mass & Electric charge $Q$ & Interaction \\
\hline$g$ & & 0 & 0 & Strong \\
$\gamma$ & & 0 & 0 & Electromagnetic \\
$W^{-}\left(W^{+}\right)$ & 1 & $80.419 \mathrm{GeV} / c^{2}$ & $-1(+1)$ & Weak \\
$Z$ & & $91.188 \mathrm{GeV} / c^{2}$ & 0 & Weak \\
\hline \hline
\end{tabular}

There are six kinds of quarks and leptons for each. Quarks are up $(u)$, down $(d)$, charm $(c)$, strange $(s)$, bottom $(b)$ and top $(t)$. Three leptons are charged leptons which are electron $(e)$, muon $(\mu)$ and tau $(\tau)$. The other three leptons are neutrinos $\left(\nu_{e}, \nu_{\mu}, \nu_{\tau}\right)$ which are neutral leptons corresponding to their charged leptons. There are anti-particles which have opposite quantum numbers for each particle.

These fermions are characterized with weak isospin $(I)$ and weak hypercharge $(Y)$. Weak hypercharge $Y$ is defined by

$$
Q=I_{3}+Y / 2,
$$

where $Q$ is the electric charge. Quarks have another quantum number which is three types color charge $(R, G$, and $B)$.

Table 1.1 summarizes the properties of quarks and leptons.

\subsubsection{Bosons}

The interaction between elementary particles is mediated by $s=1$ bosons. Gluon $(g)$ mediate strong interactions and is massless bi-colored particle. Gluon interacts only colored particles. The photon $(\gamma)$ mediates electromagnetic interactions and is a massless particle. $W^{ \pm}$and $Z$ mediate weak interactions and are massive particles. The $W^{ \pm}$and $Z$ acquire the masses by electroweak symmetry breaking through the Higgs mechanism. Table 1.2 summarizes the properties of gauge bosons. 


\subsubsection{QCD Lagrangian}

QCD satisfies strictly $S U(3)$ gauge symmetry and is not accompanied by spontaneously symmetry breaking. QCD lagrangian is given

$$
\begin{aligned}
\mathcal{L} & =\bar{\psi}\left(i \gamma^{\nu} D_{\nu}-m\right) \psi-\frac{1}{4} \boldsymbol{F}_{\mu \nu} \cdot \boldsymbol{F}^{\mu \nu} \\
D_{\mu} & =\partial_{m u}+i g \boldsymbol{A}_{\mu} \cdot \boldsymbol{t}=\partial_{\mu}+i g \sum_{k=1}^{8} A_{k \mu} \frac{\lambda_{k}}{2} \\
\boldsymbol{F}_{\mu \nu} & =\partial_{\mu} \boldsymbol{A}_{\nu}-\partial_{\nu} \boldsymbol{A}_{\mu}-g \boldsymbol{A}_{\mu} \times \boldsymbol{A}_{\nu} \\
\boldsymbol{F}_{\mu \nu} & =\left(F_{k \mu \nu} ; k=1 \sim 8\right) \\
\boldsymbol{t} & =\left(t_{k} ; k=1 \sim 8\right)
\end{aligned}
$$

,where $\psi=(R, G, B)$ which is quark color charge. $t_{k}=\lambda_{k} / 2$ is the $3 \times 3$ representation matrix of the generator. QCD is a noncommutative gauge theory, and $\boldsymbol{F}_{\mu \nu}$ includes nonliner term of $\boldsymbol{A}_{\mu}$.

\subsubsection{Electroweak Interactions}

Electroweak interaction satisfies $S U(2) \times U(1)$ gauge symmetry. There are individual hypercharge $Y$ and isospin $\left(T, T_{3}\right)$ corresponding to left-handed and right-handed fermions. That means interaction related to left-handed particles different right-handed one. The lagrangian of electroweak interaction is separated into gauge sector and higgs sector. Each sector satisfies gauge symmetry. The gauge sector represents self-energy and interactions for fermions and gauge bosons. The higgs sector represents self-energy of Higgs boson and interactions connected with Higgs boson. Self-coupling of Higgs boson produce the spontaneous symmetry breaking of vacuum. As a result, fermions and gauge bosons obtain their mass.

Here is the lagrangian which satisfies $S U(2) \times U(1)$ gauge symmetry for electron and neutrino which are doublet of $S U(2)$ symmetry.

$$
\begin{aligned}
\mathcal{L}_{\mathrm{GWS}}= & -\frac{1}{4} \boldsymbol{F}_{\mu \nu} \cdot \boldsymbol{F}^{\mu \nu}-\frac{1}{4} B_{\mu \nu} B^{\mu \nu}+\bar{\Psi}_{L} i \gamma^{\mu} D_{\mu} \Psi_{L}+\bar{e}_{R} i \gamma^{\mu} D_{\mu} e_{R} \\
& +\left(D_{\mu} \Phi\right)^{\dagger}\left(D_{\mu} \Phi\right)-V(\Phi)-f_{e}\left[\overline{e_{R}}\left(\Phi^{\dagger} \Psi_{L}\right)+\left(\overline{\Psi_{L}} \Phi\right) e_{R}\right] \\
F_{\mu \nu}= & \partial_{\mu} \boldsymbol{W}_{\nu}-\partial_{\nu} \boldsymbol{W}_{\mu}-g_{W} \boldsymbol{W}_{\mu} \times \boldsymbol{W}_{\nu} \\
B_{\mu \nu}= & \partial_{\mu} B_{\nu}-\partial_{\nu} B_{\mu} \\
D_{\mu}= & \partial_{\mu}+i g_{W} \boldsymbol{W}_{\mu} \cdot \boldsymbol{t}+i\left(\frac{g_{B}}{2}\right) B_{\mu} \cdot Y \\
V(\Phi)= & \lambda\left(|\Phi|^{2}+\frac{\mu^{2}}{2 \lambda}\right)^{2} \\
\Psi_{L}= & {\left[\begin{array}{c}
\nu_{L} \\
e_{L}
\end{array}\right] } \\
\Phi= & {\left[\begin{array}{c}
\phi^{+} \\
\phi^{0}
\end{array}\right], \quad \Phi^{c}=i \tau_{2} \Phi^{\dagger}=\left[\begin{array}{c}
\phi^{0 \dagger} \\
-\phi^{-}
\end{array}\right] }
\end{aligned}
$$

Quantities with bold outline letter are vector in isospin space that is $\boldsymbol{F}=\left(F^{a}, a=1 \sim 3\right)$. The first line on lagrangian (eq. 1.2) represents the gauge sector, while the second line represents the higgs sector. Adding $W^{0}$ and $B$ mixing and spontaneous symmetry breaking to the lagrangian, we can obtain the lagrangian of Glashow-Weinberg-Salam theory [6-8]. 


\subsubsection{Fermion Mass Term}

Higgs field obtain vacuum expectation value $v$ via spontaneous symmetry breaking, and require eq. (1.3).

$$
\Phi \rightarrow\left[\begin{array}{c}
0 \\
(v+\phi) / \sqrt{2}
\end{array}\right], \quad \Phi^{c} \rightarrow\left[\begin{array}{c}
(v+\phi) / \sqrt{2} \\
0
\end{array}\right]
$$

Inserting eq. (1.3) into third term of eq. (1.2), the mass term of fermion become

$$
\begin{aligned}
\mathcal{L}_{Y U K A W A} & =-f_{e}\left[\bar{e}_{R} e_{L}+\bar{e}_{L} e_{R}\right](v+\phi) / \sqrt{2} \\
& =-m_{e} \bar{e} e-\left(m_{e} / v\right) \bar{e} e \phi \\
m_{e}=f_{e} v / \sqrt{2} . &
\end{aligned}
$$

We can make d quark $\left(T_{3}=-1 / 2\right)$ mass using same procedure only changing coupling constant $f$. To generate u quark mass, we need to choose $\Phi^{c}$.

\subsubsection{Mass Term of $W$ and $Z$}

We can obtain mass term of $W^{+}, W^{-}$and $Z$ boson to make the replacement

$$
\Phi \rightarrow\left[\begin{array}{c}
0 \\
v / \sqrt{2}
\end{array}\right]
$$

on the kinetic energy term of the higgs sector which is the first term of the second line of eq. (1.2). Then $W^{ \pm}$and $Z$ mass are

$$
m_{W}=\frac{g_{W} v}{2}, \quad m_{Z}=\frac{g_{Z} v}{2}=\frac{m_{W}}{\cos \theta_{W}}
$$

, where $g_{W}$ is $g_{W}=e / \sin \theta_{W}, \sin \theta_{W} \simeq 0.234$ and $g_{Z}$ is $g_{\mathrm{Z}}=e / \sin \theta_{W} \cos \theta_{W}$. Following notation describe the relation between Weak interaction and fermi coupling constant.

$$
\begin{aligned}
\frac{G_{F}}{\sqrt{2}} & =\frac{g_{W}^{2}}{8 m_{W}^{2}}=\frac{e^{2}}{8 m_{W}^{2} \sin ^{2} \theta_{W}} \\
v & =\frac{2 m_{W}}{g_{W}}=\left(\sqrt{2} G_{F}\right)^{-1 / 2} \simeq 246 \mathrm{GeV}
\end{aligned}
$$

\subsubsection{Higgs Interaction}

The lagrangian for the higgs interaction is described by inserting eq. (1.3) into the higgs sector of eq. (1.2).

$$
\begin{aligned}
\mathcal{L}_{H}= & \frac{1}{2}\left(\partial_{\mu} \phi \partial^{\mu} \phi-m_{H}^{2} \phi^{2}\right)-\lambda v \phi^{3}-\lambda \frac{\phi^{4}}{4} \\
& -\left(\sqrt{2} G_{F}\right)^{\frac{1}{2}} \sum_{f} m_{f} \phi \bar{f} f \\
& +2\left(\sqrt{2} G_{F}\right)^{\frac{1}{2}} \phi\left(m_{W}^{2} W_{\mu}^{+} W^{-\mu}+\frac{m_{Z}^{2}}{2} Z_{\mu} Z^{\mu}\right) \\
& +\sqrt{2} G_{F} \phi^{2}\left(m_{W}^{2} W_{\mu}^{+} W^{-\mu}+\frac{m_{Z}^{2}}{2} Z_{\mu} Z^{\mu}\right) \\
m_{H}^{2}= & -2 \mu^{2}=2 \lambda \mu
\end{aligned}
$$

where $\lambda$ is a empirical value must be input. 


\subsubsection{Higgs Boson Production and Decay}

At the Tevatron, a next-to-leading-order (NLO) Higgs boson production cross section is shown in Figure 1.1. Gluon fusion Higgs boson production $(g g \rightarrow H)$ has largest cross section at the

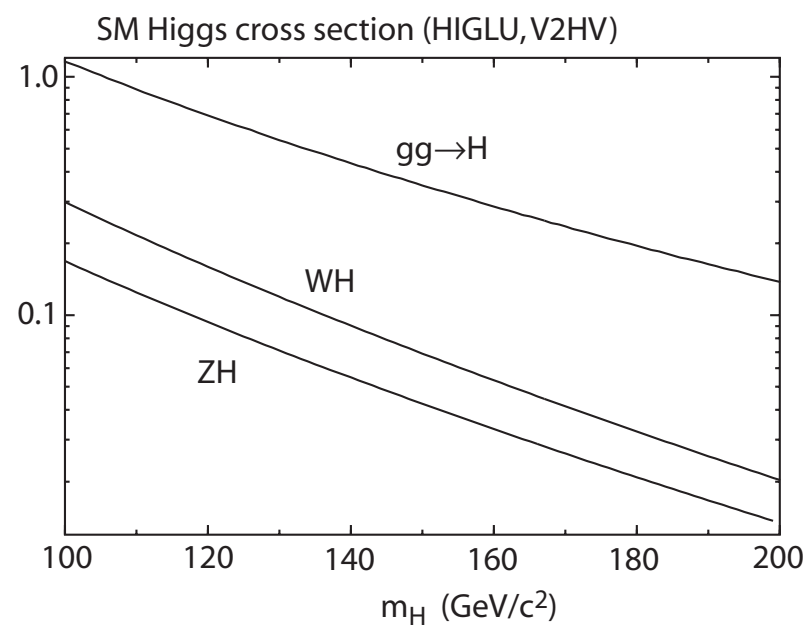

Figure 1.1: The NLO Standard Model Higgs boson production cross section in pb at the Tevatron with $p \bar{p}$ collision at $\sqrt{s}=1.96 \mathrm{TeV}$.

Tevatron. Higgs boson production in association with a $W$ boson $(W H)$ or a $Z$ boson $(Z H)$, and the cross section of $W H$ or $Z H$ process are about 10 times less than $g g \rightarrow H$ production.

The Higgs boson decay branching ratio is calculated by HDECAY [9], which can include NLO effect, and is shown in Figure 1.2. At the low mass region $\left(M_{H}<135 \mathrm{GeV} / c^{2}\right)$, Higgs boson decays mainly into $b \bar{b}$. On the other hand, $H \rightarrow W^{+} W^{-}$is dominant decay mode at the high mass region $\left(M_{H}>135 \mathrm{GeV} / c^{2}\right)$. At the low mass region, $g g \rightarrow H \rightarrow b \bar{b}$ is the predominant channel, however, this channel suffers from huge QCD multi-jet backgrounds. As a result, $W H \rightarrow \ell \nu b \bar{b}$ is one of the most sensitive channels for the low mass Higgs searches. At the high mass region, $g g \rightarrow H \rightarrow W W \rightarrow \ell \nu \ell \nu$ channel (Figure 1.3 ) gives clean search sample. $g g \rightarrow H \rightarrow W W \rightarrow \ell \nu j j$ channel (Figure Figure 1.4) is also promising decay mode. There is huge QCD multi-jet background but $W W \rightarrow \ell \nu j j$ decay mode has six times lager branching ratio than $W W \rightarrow \ell \nu \ell \nu$ decay mode. Then we focus on $g g \rightarrow H \rightarrow W W \rightarrow \ell \nu j j$ decay mode to search for the Standard Model Higgs boson. Figure 1.4 shows $g g \rightarrow H$ production and decay into a $\ell \nu j j$ final state. 


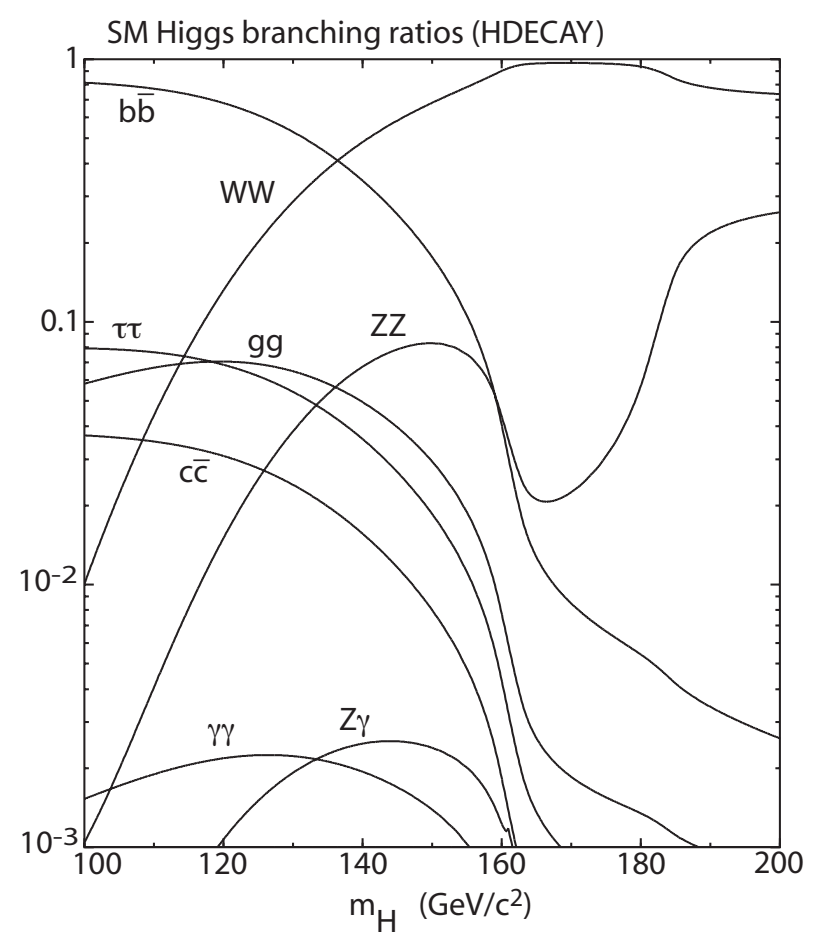

Figure 1.2: The branching ratio for each Higgs boson decay mode as a function of Higgs boson mass. 


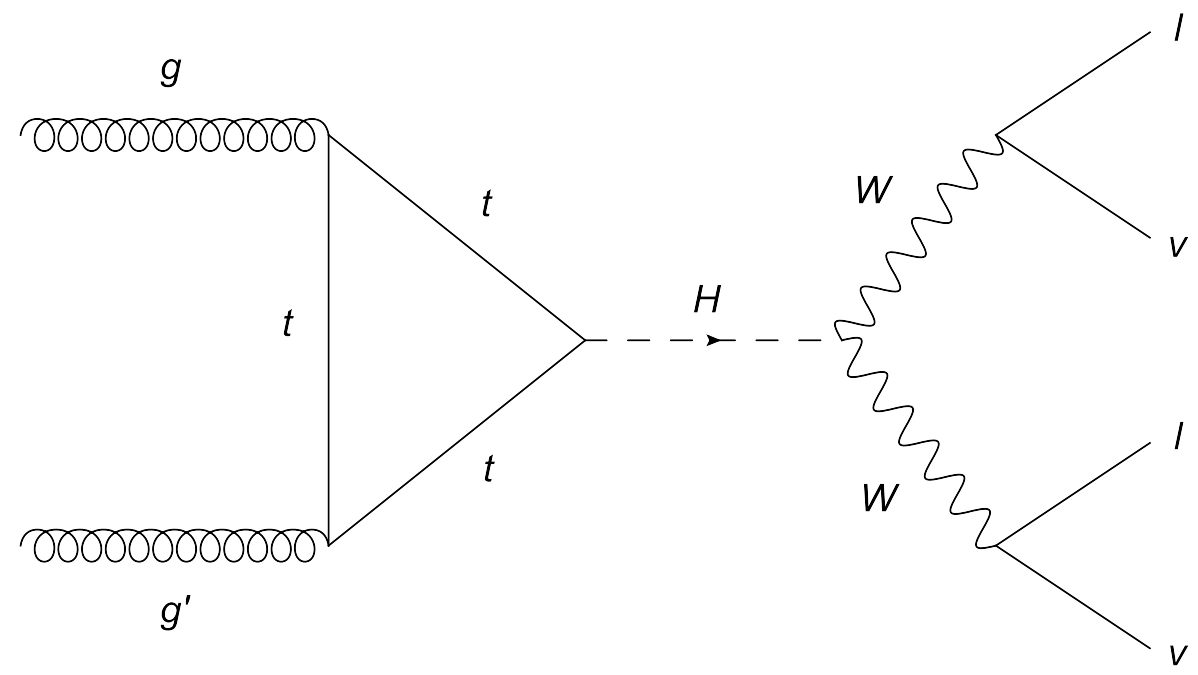

Figure 1.3: Feynman diagram of the $g g \rightarrow H \rightarrow W W \rightarrow \ell \nu \ell \nu$ channel.

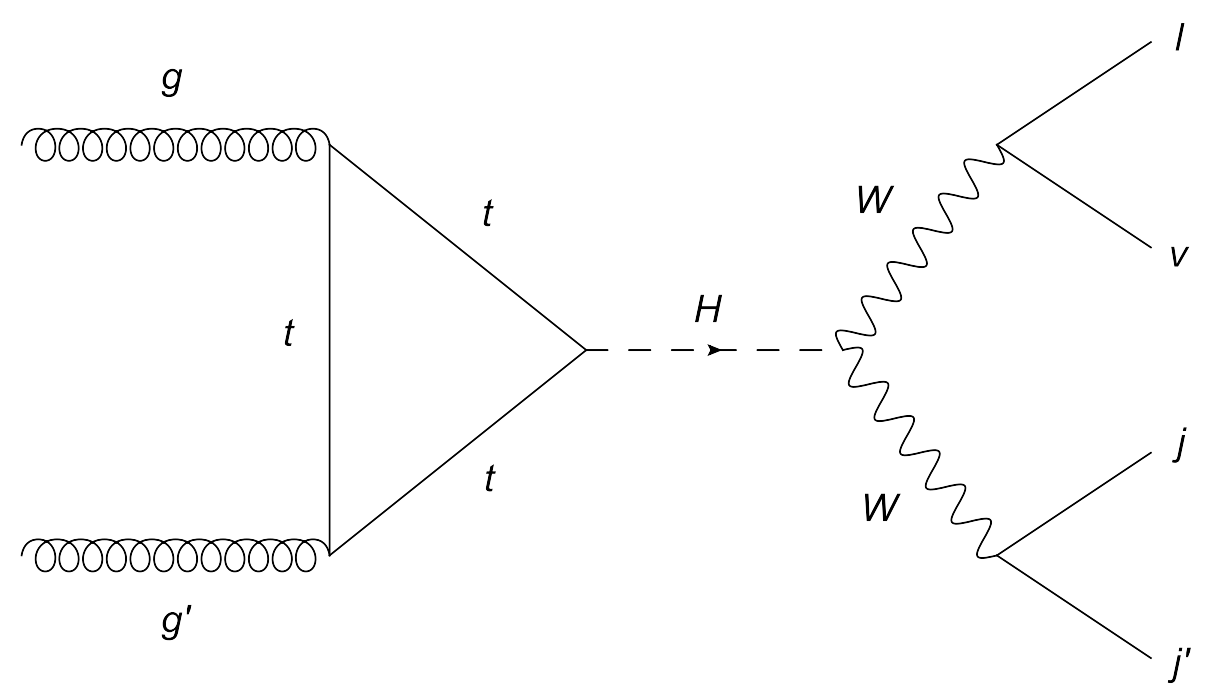

Figure 1.4: Feynman diagram of the $g g \rightarrow H \rightarrow W W \rightarrow \ell \nu j j$ channel. 


\subsection{Previous Higgs Boson Searches}

\subsubsection{Direct Searches}

\section{Searches at LEP}

The Large Electron Positron Collider (LEP) experiment at CERN performed a search for the Standard Model Higgs boson in $e^{+} e^{-}$collisions at various center-of-mass energies of 189-209 GeV [10]. At the LEP, the main search channel is $e^{+} e^{-} \rightarrow Z H$ with $Z$ boson decaying into all and Higgs boson decaying into $b \bar{b}$. Using the full data accumulated at four experiments (ALEPH, DELPHI, L3, and OPAL) from 1989 until 2000, the experiment excluded the Higgs boson mass below $114.4 \mathrm{GeV} / c^{2}$ at $95 \%$ confidence level (C.L.) (Figure 1.5).

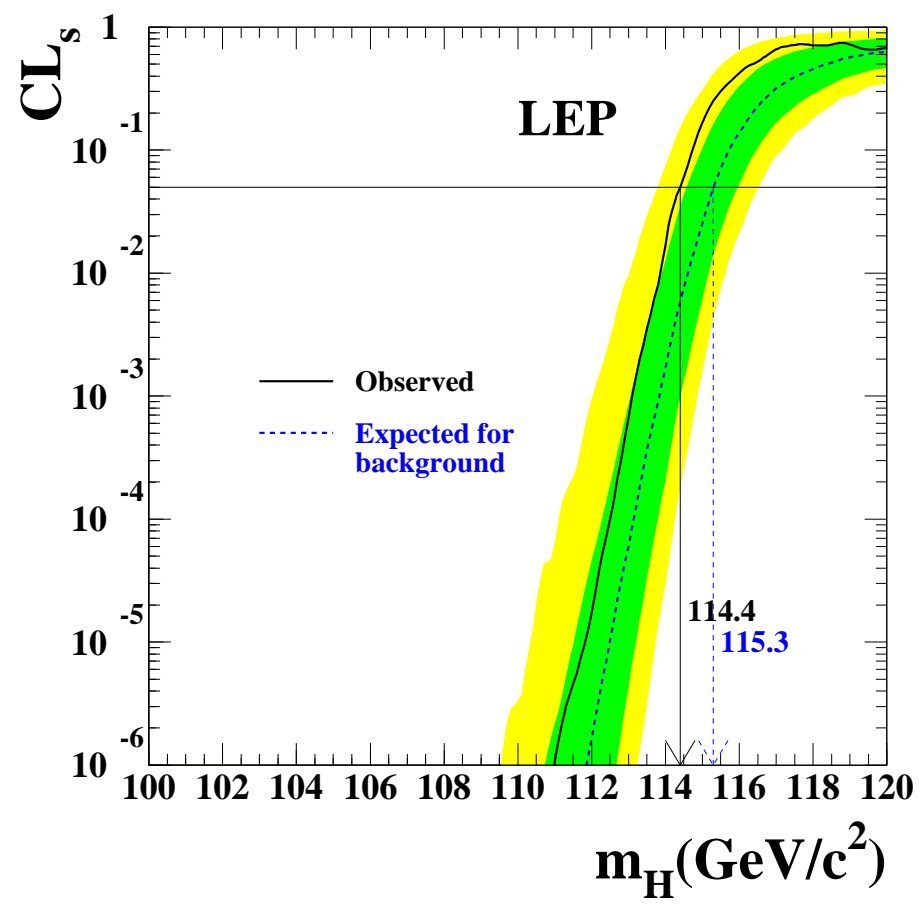

Figure 1.5: The ratio $C L_{s}=C L_{s+b} / C L_{b}$ for the signal plus background hypothesis as a function of the Higgs mass. Solid line: observed result; dashed line: median expectation. The green and yellow bands around the median expectation line correspond to the $1 \sigma$ and $2 \sigma$ probability bands.

\section{Searches at the Tevatron}

The following searches are performed at the Tevatron. There are public information of the detail of the searches on the CDF and D0 public web page [11,12].

1. The high mass Higgs boson searches (mainly $M_{H}>135 \mathrm{GeV} / c^{2}$ ) with $H \rightarrow W W^{*}$ and $W H(Z H) \rightarrow W(Z)+W W^{*}$ decays.

- same sign dilepton channel is the most sensitive channel for the high mass Higgs boson searches 
- opposite sign dilepton channel is based on same sign dilepton identification with very small background but also small effective cross section.

2. The low mass Higgs boson searches (mainly $M_{H}<135 \mathrm{GeV} / c^{2}$ ) with $H \rightarrow b \bar{b}, \tau^{+} \tau^{-}$, or $\gamma \gamma$ decays.

- $W H \rightarrow \ell^{ \pm} \nu b \bar{b}$ channel has a relatively large effective cross section.

- $Z H \rightarrow \ell^{+} \ell^{-} b \bar{b}$ channel provides clean signal among the low mass Higgs boson searches.

- $V H \rightarrow \nu \nu b \bar{b}$ channel: The Higgs boson is produced in association with a $W^{ \pm}$or $Z$ boson. $Z \rightarrow \nu \nu$ is the main decay mode, but also comes from the case that a charged lepton is not detected in the $W \rightarrow \ell \nu$ decay.

- $t t H \rightarrow$ lepton + jets channel: The Higgs boson is produced in association with $t \bar{t}$. The final state has one lepton and at least four jets.

- $t t H$ to no lepton channel: The Higgs boson is produced in association with $t \bar{t}$. This search focuses MET + jets and all hadronic decay mode.

- $q \bar{q} b \bar{b}$ channel: The Higgs boson is produced in association with a $W^{ \pm}$or $Z$ boson, or also produced through the weak vector boson fusion process. This channel suffers from the enormous QCD backgrounds.

- $\tau^{+} \tau^{-}+$jets channel: The Higgs boson is produced through three processes: gluon fusion, associated with a $W$ or $Z$ boson, or weak vector boson fusion. The lower branching fraction of $H \rightarrow \tau^{+} \tau^{-}$can be recovered with using multiple production processes.

- $\gamma \gamma$ channel: The Higgs boson is produced through the gluon fusion process and decays into two photons. Due to the very small branching fraction of $H \rightarrow \gamma \gamma$, this channel has a very small effective cross section

Figure 1.6 summarizes the CDF combined 95\% C.L. upper limit on the Higgs boson production cross section normalized to the Standard Model expectation. The CDF experiment excludes the ranges with SM Higgs boson with mass $m_{H}$ between 156.5 and $173.7 \mathrm{GeV} / \mathrm{c}^{2}$, and between 100 and $104.5 \mathrm{GeV} / \mathrm{c}^{2}$. Figure 1.7 summarizes the Tevatron combined $95 \%$ C.L. upper limit. The Tevatron combined result is that the mass range $m_{H}$ between 156 and $177 \mathrm{GeV} / \mathrm{c}^{2}$, and between 100 and $108 \mathrm{GeV} / \mathrm{c}^{2}$ are excluded. 


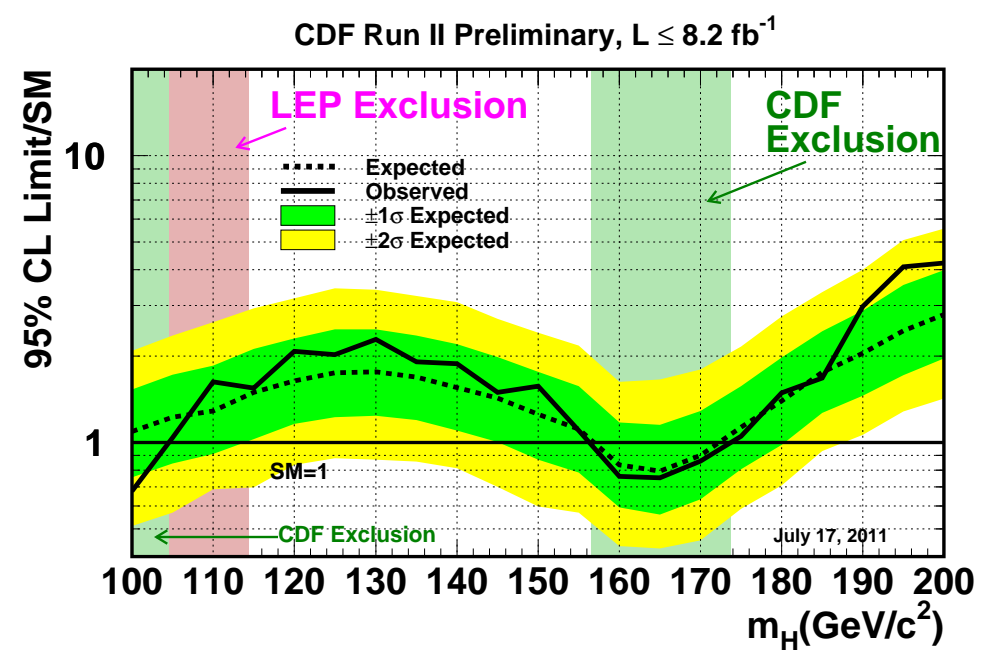

Figure 1.6: The summary of the CDF combined $95 \%$ upper limit on the Higgs boson production cross section normalized to the Standard Model expectation. The CDF experiment exclude the ranges with SM Higgs boson with mass $m_{H}$ between 156.5 and $173.7 \mathrm{GeV} / \mathrm{c}^{2}$, and between 100 and $104.5 \mathrm{GeV} / \mathrm{c}^{2}$.

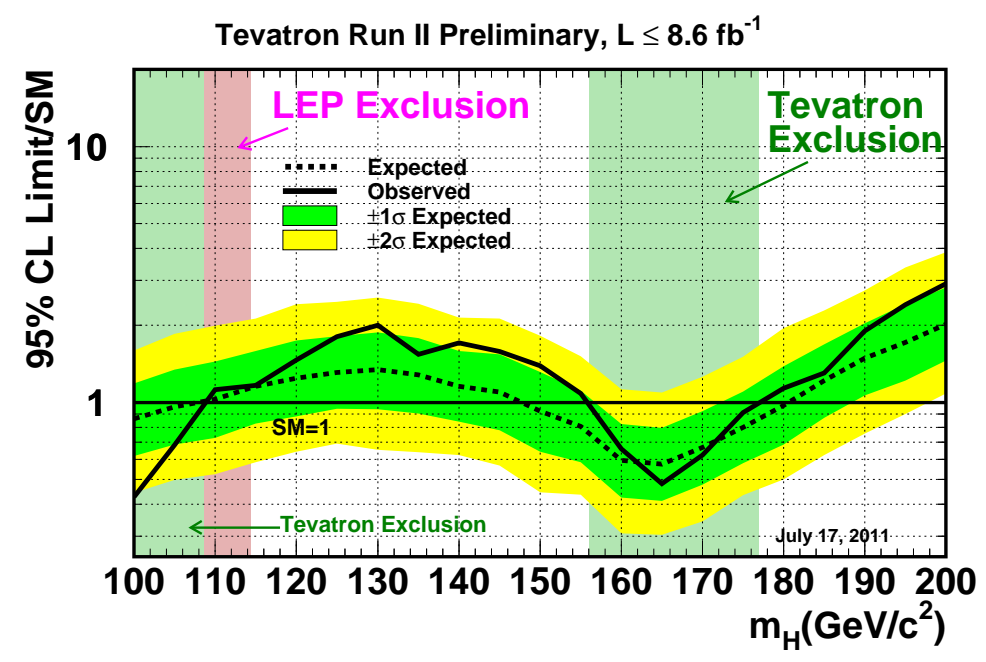

Figure 1.7: The summary of the Tevatron combined the Higgs boson search. 95\% C.L. exclusion for Standard Model Higgs boson with mass $m_{H}$ between 156 and $177 \mathrm{GeV} / \mathrm{c}^{2}$, and between 100 and $108 \mathrm{GeV} / \mathrm{c}^{2}$. 


\subsubsection{Indirect Searches}

The Higgs boson mass is not theoretically predicted, but it is possible to estimate using theoretical considerations and precision electroweak parameter measurements. The Higgs boson contributes to the propagator of the $W$ and $Z$ bosons through loop-effects. The corrections to the $W$ and $Z$ masses are logarithmic functions of the Higgs mass leading to limited conclusions:

$$
\Delta_{r}=\frac{3 G_{F}}{8 \pi^{2} \sqrt{2}} m_{t}^{2}+\frac{\sqrt{2} G_{F}}{16 \pi^{2}} m_{t}^{2}\left[\frac{11}{3} \ln \left(\frac{m_{h}^{2}}{m_{W}^{2}}\right)+\cdots\right]+\cdots
$$

where the radiative corrections $\Delta_{r}$ is defined as:

$$
\frac{m_{W}^{2}}{m_{Z}^{2}}+\sin ^{2} \theta_{W} \equiv 1+\Delta r
$$

As shown in the above equation, the constraint on the Higgs mass is mainly related with the top quark and the $W$ boson masses. The Electroweak working group [13] has been performing global fits to the electroweak data, with the current most precision measurement of $m_{t}=173.2 \pm$ $0.9 \mathrm{GeV} / c^{2}$ and $m_{W}=80.399 \pm 0.023 \mathrm{GeV} / c^{2}$. Figure 1.8 shows the $\Delta \chi^{2}$ curve as a function of the $m_{H}$ derived from global fits. The preferred Higgs mass from this fit corresponding to the minimum of the curve is

$$
m_{H}=92_{-26}^{+34} \mathrm{GeV} / c^{2}
$$

and the $95 \%$ confidence level upper limit is

$$
m_{H}=161 \mathrm{GeV} / c^{2}
$$

The upper limit increases to $185 \mathrm{GeV} / c^{2}$ when including the LEP direct search limit of 114.4 GeV $/ c^{2}$. The recent combined result at the Tevatron (CDF and D0) excludes the mass range between 156 and $177 \mathrm{GeV} / c^{2}$ at $95 \%$ confidence level, which is also shown in yellow. 


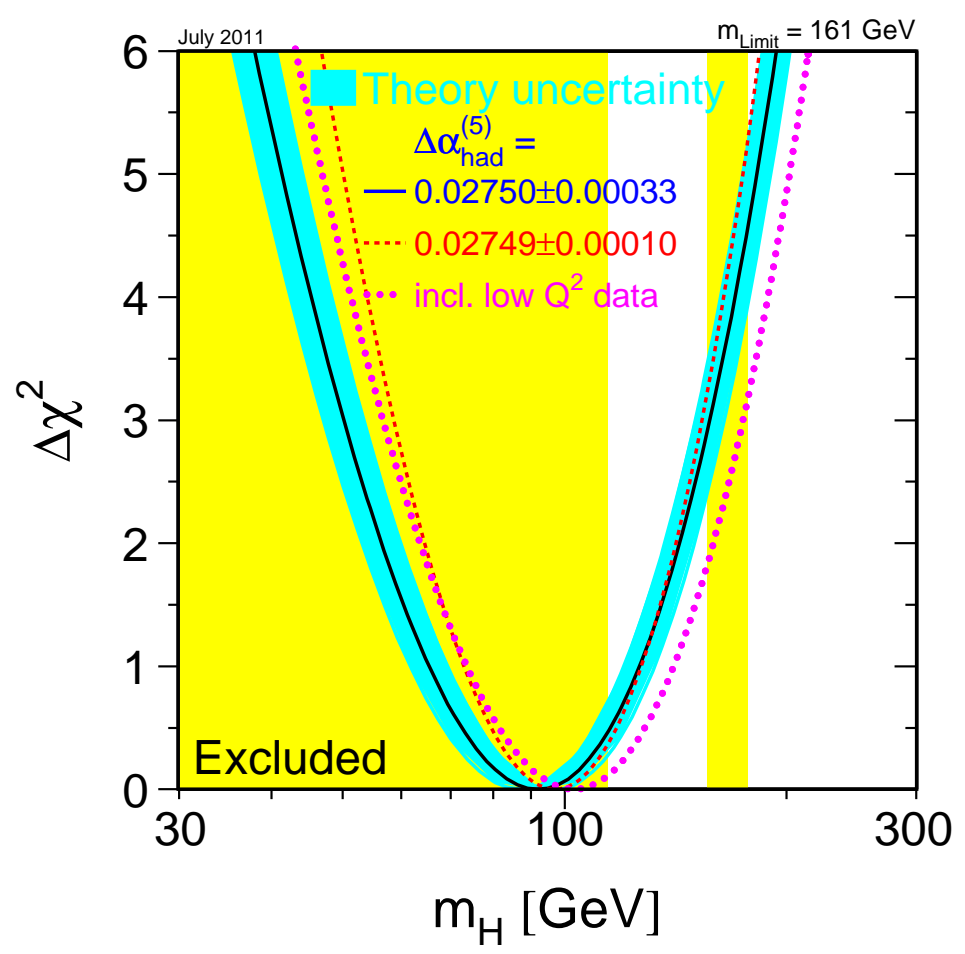

Figure 1.8: The $\Delta \chi^{2}$ distribution as a function of the Higgs mass. This electroweak global fit is based on the precision measurement at LEP, SLD, Tevatron, and NuTev. 


\section{Chapter 2}

\section{Experimental Apparatus}

This chapter describes the Fermilab accelerator complex, CDF II detector components, trigger and data acquisition (DAQ) system.

\subsection{Characteristics of Fermilab Accelerators}

The Fermilab accelerator complex is constructed by the various accelerators which are the Preaccelerator, Linac and Booster, Main Injector, Recycler, Tevatron, Debuncher and Accumulator. The Pre-accelerator, Linac and Booster are collectively known as the Proton Source. The Debuncher and Accumulator are referred to as the Antiproton Source. The Tevatron is the last in the chain of accelerators collides protons and antiprotons.

The Tevatron design is vastly simplified if it collides protons with antiprotons because the protons can travel opposite directions in the same beam-pipe and be bent by the same set of magnets. An overview of the entire accelerator complex is shown in Figure 2.1. The Tevatron's first physics run, referred to as Run I, occurred from 1992-1996. After a series of upgrades, it began running again, referred to as Run II, in 2002 and is shut down in September 2011.

\section{Preaccelerator}

The preaccelerator is really the first accelerator. It is the source of the negatively charged hydrogen ions accelerated by the linear accelerator. The preaccelerator is a Cockcroft-Walton-style [14] electrostatic accelerator which consists of the source housed in an electrically charged dome. The source converts hydrogen gas to ionized hydrogen gas $(\mathrm{H}-)$. The dome is charged to a potential of $-750 \mathrm{KeV}$. The ionized gas is allowed to accelerate through a column from the charged dome to the grounded wall to an energy of $750 \mathrm{KeV}$. After beam exits the accelerating column, it travels through a transfer line and then enters the Linac.

\section{Linac}

The Linear Accelerator is the next level of acceleration for the negatively charged hydrogen ions. It takes the ions with an energy of $750 \mathrm{KeV}$ and accelerates them to an energy of $400 \mathrm{MeV}$. The Linac consists of two main sections, the low energy drift tube Linac and the high-energy side coupled cavity Linac. The first section consists of five drift tubes, modeled after Luis Alvarez' original proton linear accelerator, that accelerate the ion beam to $117 \mathrm{MeV}$. The second section has seven side-coupled cavity modules that accelerate the $\mathrm{H}^{-}$ions to $400 \mathrm{MeV}$. At the far end of 


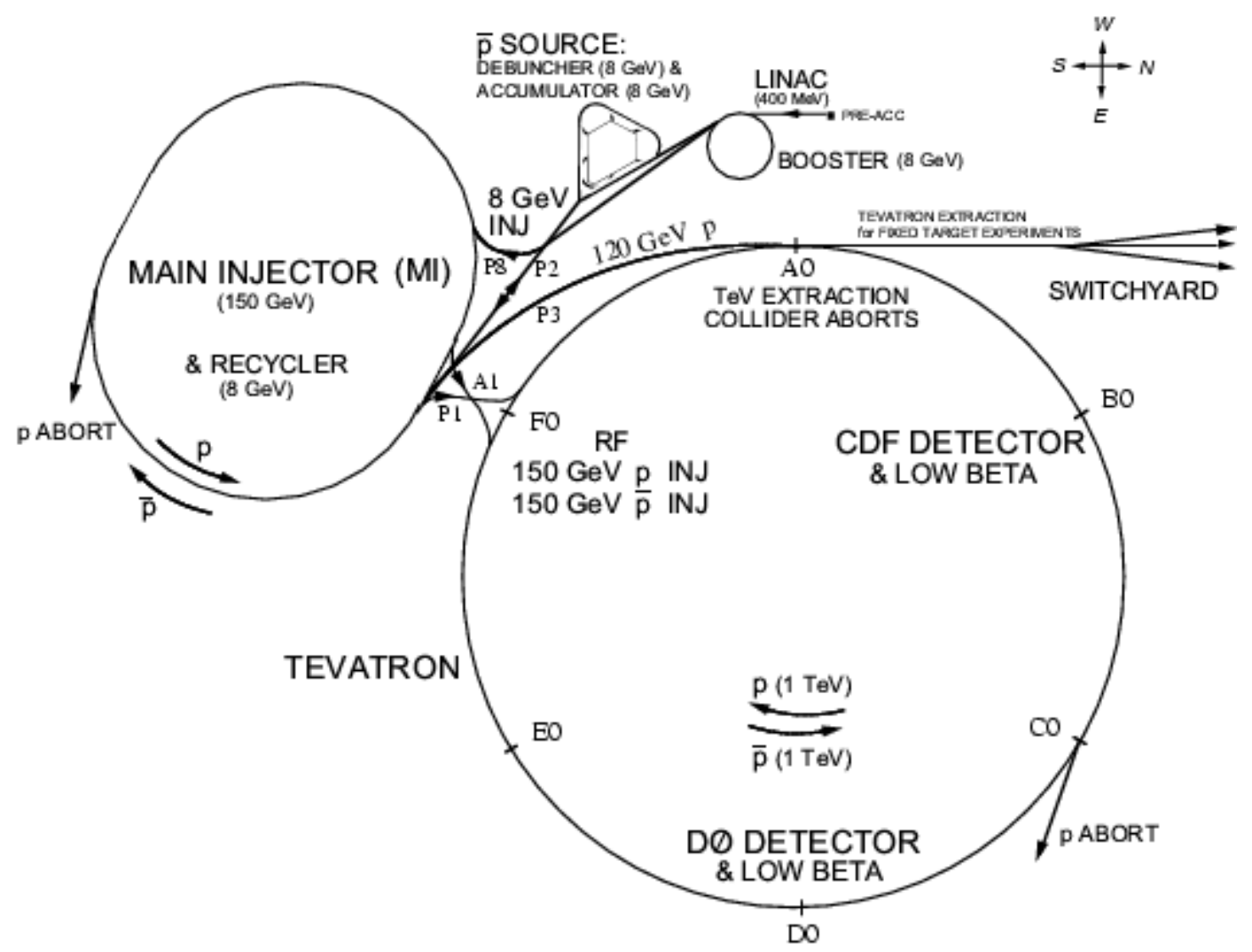

Figure 2.1: A diagram of the Fermilab accelerator chain.

the Linac is a chopper, that electrostatically selects a portion of the Linac beam to be sent along a transfer line to the Booster. The Linac completes fifteen acceleration cycles per second.

\section{Booster}

The Booster is the next level of acceleration. It takes the $400 \mathrm{MeV}$ negative hydrogen ions from the Linac and strips the electrons off, which leaves only the proton, and accelerating the protons to $8 \mathrm{GeV}$. The Booster is a proton synchrotron, approximately $150 \mathrm{~m}$ in diameter. It has the same duty cycle as the Linac, $15 \mathrm{~Hz}$. The acceleration is accomplished by eighteen ferrite-tuned RF cavities located around the ring. Ninety-six conventional magnets with a maximum field of $0.7 \mathrm{~T}$ bend the beam into a circular orbit. The Booster is able to hold multiple batches of particles from the Linac at once to increase beam intensities, often storing eleven or twelve batches in its ring.

A special set of magnets handles the injection of incoming $\mathrm{H}^{-}$ions from the Linac. Magnets bend the circulating protons and the injected $\mathrm{H}^{-}$ions into a single beam that points through a sheet of foil. The foil strips the electrons from the $\mathrm{H}^{-}$ions, leaving behind only protons. A similar set of magnets steers the beam back into the Booster orbit while removing any leftover $\mathrm{H}^{-}$ions.

A set of fast kicker magnets extracts the proton beam from the Booster. The protons go into a transfer line that leads to the Main Injector. 


\section{Main Injector}

The Main Injector is a large proton synchrotron with a diameter of about $1 \mathrm{~km}$. It has two main functions involving the Tevatron: accelerating protons and antiprotons to $150 \mathrm{GeV}$ for injection into the Tevatron, and accelerating protons to $120 \mathrm{GeV}$ to be sent to the antiproton source.

The Main Injector uses 344 dipole magnets and 208 focusing quadrupole magnets, all conventional water-cooled electromagnets, to steer the proton beam. It can accelerate protons to $150 \mathrm{GeV}$ in two seconds.

\section{Tevatron}

The Tevatron is a large synchrotron, $1 \mathrm{~km}$ in radius, that accelerates particles from $150 \mathrm{GeV}$ to $980 \mathrm{GeV}$. It keeps both protons and antiprotons in the same beampipe, revolving in opposite directions. Electrostatic separators produce a strong electric field that keeps the two beams from touching except at the collision point. The beam is steered by 774 superconducting dipole magnets and 240 quadrupole magnets with a maximum magnetic field of $4.2 \mathrm{~T}$. They are cooled by liquid helium to $4.2 \mathrm{~K}$, at which point the niobium-titanium alloy in the magnets becomes superconducting.

The Tevatron holds 36 bunches each of protons and antiprotons. The process of injecting particles into the machine, accelerating them, and initiating collisions, referred to as a shot, starts with injection of protons, one bunch at a time, at $150 \mathrm{GeV}$ from the Main Injector. The antiprotons are injected four bunches at a time from the Recycler through the Main Injector. RF cavities accelerate the beams to $980 \mathrm{GeV}$, and then some electrostatic separators switch polarity to cause the beams to collide at two points. Each collision point lies at the heart of a particle detector: one named D0 and the other named the Collider Detector at Fermilab (CDF).

\section{Antiproton Source}

The antiproton source produces antiprotons for use in Tevatron collisions. The Main Injector sends $120 \mathrm{GeV}$ protons down a transfer line to a nickel target. (Nickel was chosen because it can absorb more heat without melting than other metals.) Antiprotons are among the products resulting from this collision; they are selected by an electromagnetic selector and focused down a transfer line to the Debuncher. Studies have shown that $120 \mathrm{GeV}$ is the optimal energy for antiproton production; at this energy, approximately one antiproton is collected per $10^{5}$ protons sent to the antiproton source. The resultant antiprotons have an average energy of about $8 \mathrm{GeV}$.

\section{Debuncher}

The Debuncher is not an accelerator but a triangular storage ring. Its main purpose is to "debunch" the particle beam, removing its RF bunch structure. Magnets in the Debuncher decrease the momentum spread of the antiprotons by rotating them in phase space, trading momentum spread for time spread. This results in a beam of particles that have no RF bunch structure but have roughly uniform momentum. Antiprotons remain in the Debuncher until the next batch of protons is sent to the antiproton target, at which point the antiprotons are sent to the Accumulator.

\section{Accumulator}

The Accumulator lies in the same tunnel as the Debuncher. It is a long term antiproton storage ring, designed to store antiprotons with minimal losses for days. Antiprotons from the Debuncher are 
manipulated by RF systems in the Accumulator to fill a stable region of phase space, known as the core. The core is kept as small as possible to minimize the momentum spread of the antiprotons; a smaller beam gives a higher luminosity upon injection into the Tevatron.

While the antiprotons stay in the Accumulator, they are reduced in transverse momentum through a process called stochastic cooling [15]. This procedure measures the momentum spread of a group of antiprotons and sends a signal across the ring to corrector magnets, which adjust their fields for each group of particles to reduce the momentum spread of those particles. This results in denser antiproton beams injected into the Tevatron, increasing the resulting luminosity.

Extraction from the Accumulator requires the antiprotons to be collected into bunches again. Adiabatic activation of RF stations causes a portion of the beam to be collected into bunches, which are then transferred back to the Main Injector, decelerated to $8 \mathrm{GeV}$, and injected into the Recycler.

\section{Recycler}

When the Accumulator reaches its maximum optimal capacity, its antiprotons are passed into the Recycler, a ring of permanent magnets in the same tunnel as the Main Injector. This storage ring keeps antiprotons at $8 \mathrm{GeV}$, collecting them until the Tevatron is ready for injection. In the Recycler, antiprotons are cooled further using a process called electron cooling [16], in which a beam of electrons is accelerated to the same energy as the antiprotons and runs alongside them. Transverse momentum from the antiproton beam is passed to the much lighter electrons, causing the antiprotons to lose transverse momentum, making the beam smaller. Antiprotons are injected from the Recycler to the Main Injector, which accelerates them to $150 \mathrm{GeV}$ for injection into the Tevatron.

\subsection{The CDF II Detector}

The CDF II detector is a general purpose detector designed to study $p \bar{p}$ collisions at the Tevatron. It was commissioned in the beginning of Run II in 2001. The detector components are arranged in cylindrical shape. The position of the sub-detectors are described in cylindrical coordinates $(r, \phi, z)$ with fixing the origin to the geometric center of the detector. The $\phi=0$ is parallel to the ground and points out of the Tevatron ring. The z-axis points along the beam in the direction of the protons.

The outgoing particles are described in spherical coordinates. The z-axis is replaced by the polar angle $\theta$. In the event reconstruction, the transverse momentum of particles $p_{T}=|\boldsymbol{p}| \sin \theta$ is measured in the transverse plane, and their direction is given by the pseudorapidity $\eta$. The pseudo-rapidity is defined as

$$
\eta=-\ln \tan \left(\frac{\theta}{2}\right)
$$

which is a good approximation at high energies $\left(p_{T} \gg m\right)$ to the rapidity

$$
y=\frac{1}{2} \ln \left(\frac{E+p_{z}}{E-p_{z}}\right)
$$

where $E$ is the particle's energy and $p_{z}$ is its momentum along the z-axis.

A solid cutaway view of the CDF II detector is shown in Fig. 2.2. In the center of the detector, the charged particle tracking system is enclosed by a superconducting solenoid. Outside the solenoid is the calorimeter system which is surrounded by the muon detectors. 


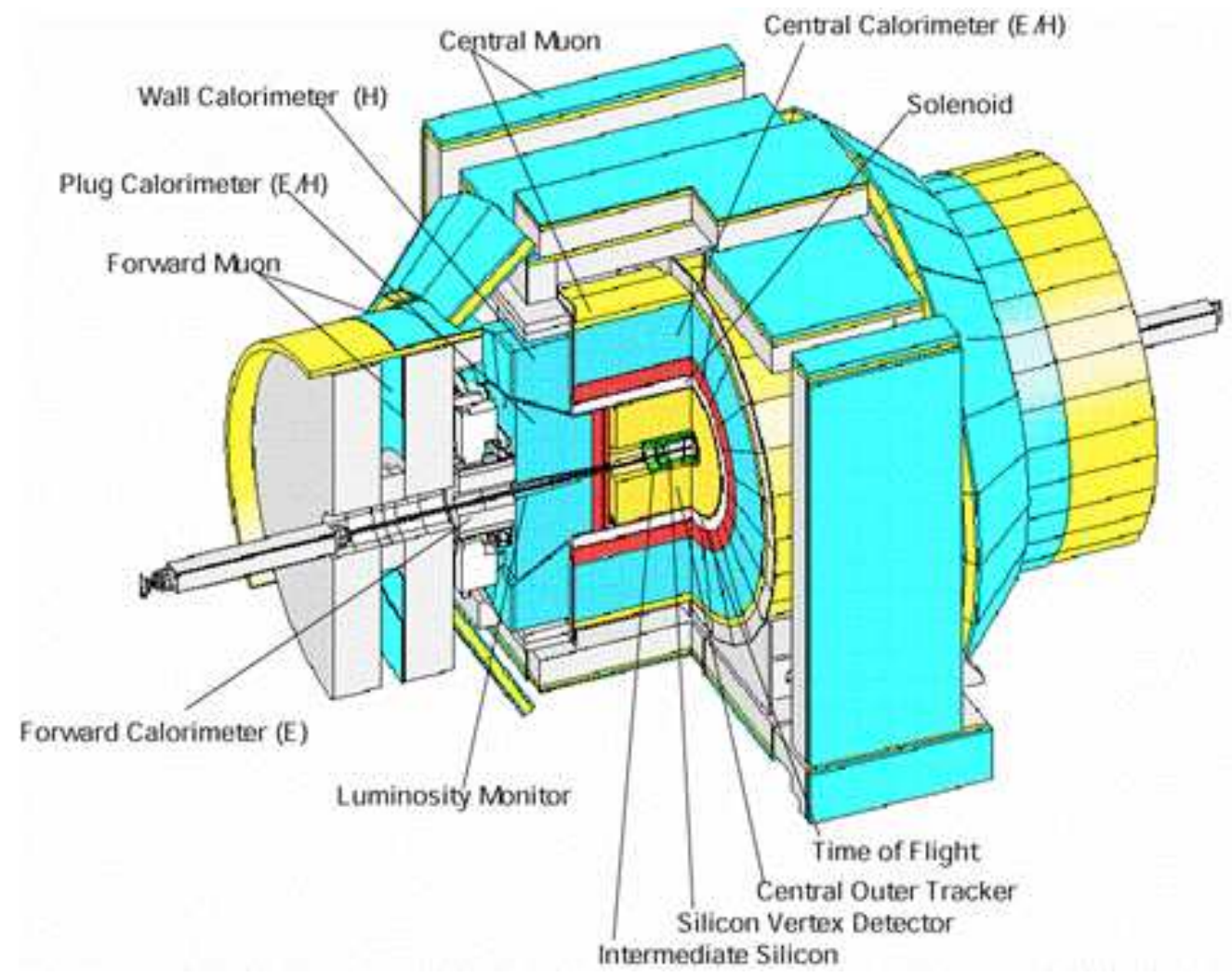

Figure 2.2: Isometric view of the CDF II detector.

\subsubsection{Tracking System}

The innermost part of the CDF II detector is the tracking system which is composed of multilayer silicon microstrip detectors, an open-cell wire drift chamber, and a superconducting solenoid magnet. The coverage of these detectors are illustrated in Figure 2.3. It is used to reconstruct trajectories of charged particles and precisely measure their momenta. The reconstructed particle trajectories are called tracks. Good resolution is required to detect displaced secondary vertices, which is a key to detect $B$ hadrons.

\section{Silicon Detectors}

The silicon detector consists of three parts: Layer 00 (L00) [17], the Silicon Vertex Detector (SVXII) [18], and the Intermediate Silicon Layers (ISL) [19], as shown in Figure 2.4. The detector offers full tracking coverage for $|\eta|<2.0$ as shown in Figure 2.4 (left).

All the three sub-detectors are constructed from wafers of n-type silicon with thin strips ( $\sim 10 \mu \mathrm{m})$ doped with p-type silicon (n-type in addition for SVXII sensors). The reverse bias voltage extends the depletion region from the p-n junction. When a charged particle passes through the depleted region, it ionizes the silicon wafer creating electron and hole pairs. The voltage moves electrons to one side of the sensor, the holes to the other side. Then, induced charge is read out by ASIC chips mounted at the end of the sensors. The spacial resolution is varying depending on each silicon sub-detector, since pitches are ranging from $25 \mu \mathrm{m}$ to more than $100 \mu \mathrm{m}$.

L00: The L00, the innermost silicon detector, consists of one layer of single-sided silicon attached 


\section{CDF Tracking Volume}

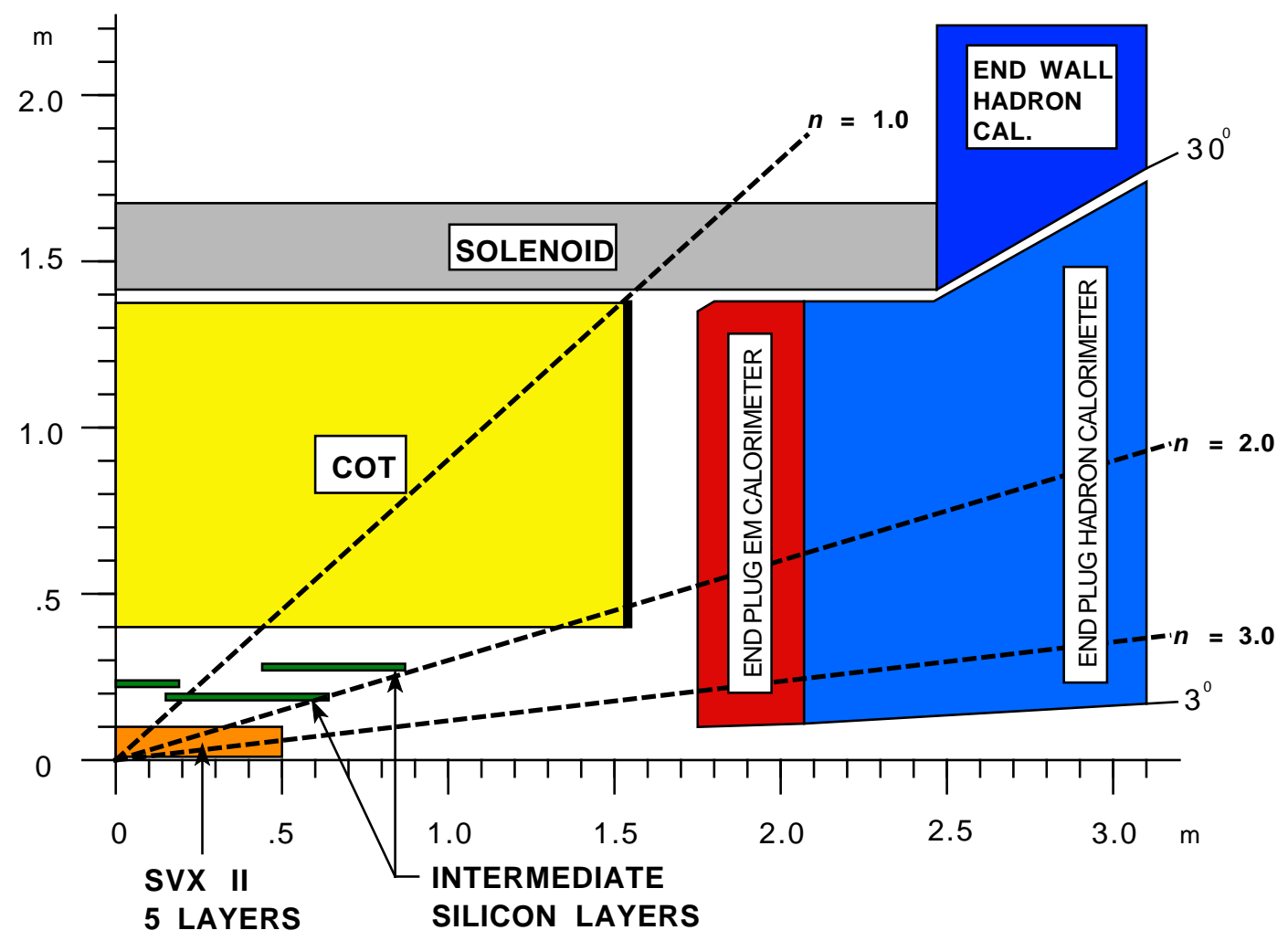

Figure 2.3: The CDF tracking system.

directly to the beampipe, only at $\sim 1.5 \mathrm{~cm}$ radius. Its purpose is to improve the resolution of the track impact parameter and position of secondary vertices.

SVXII: The SVXII is the main part of the silicon detector, which consists of five concentric layers of double-sided silicon. These layers are placed at radii from $2.4 \mathrm{~cm}$ to $10.7 \mathrm{~cm}$. The hit information of the SVXII provides high resolution tracking information and is especially useful for reconstructing displaced secondary vertices.

ISL: The ISL is the outermost silicon detector, which consists of a single layer at $|\eta|<1.0$ (at a radius of $22 \mathrm{~cm}$ ) and two layers at $1.0 \leq|\eta| \leq 2.0$ (at a radius of $20 \mathrm{~cm}$ and $29 \mathrm{~cm}$ ). This detector helps the connection of tracks between the Central Outer Tracker and the SVXII. This improves the track resolution and the performance of forward tracking in $|\eta| \leq 2.0$.

\section{Central Outer Tracker (COT)}

The COT is a cylindrical drift chamber that sits directly outside of the silicon detectors in the central region $(|\eta|<1.0)$ [20]. The chamber consists of eight cylindrical radial sections ("superlayers") of $310 \mathrm{~cm}$ long cells at radii between 40 and $132 \mathrm{~cm}$ from the detector center. The eight superlayers are placed in alternating axial and stereo sections: wires in axial superlayers run parallel to the $z$-axis, while wires in stereo superlayers are strung at \pm 2 degree angles with respect to the $z$-axis. The number of cells in the superlayer increases radially outwards, the innermost 


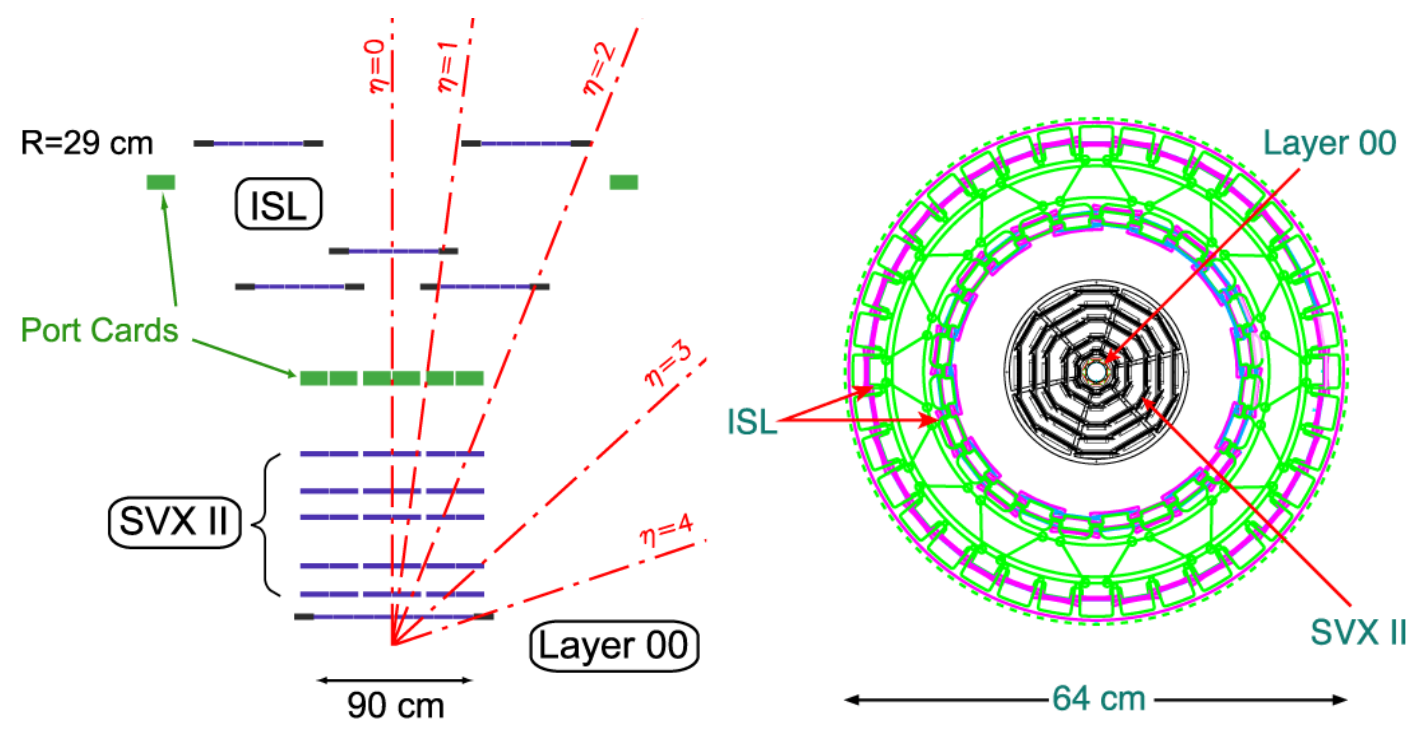

Figure 2.4: The CDF silicon detector. Left: the coverage of the silicon detector in r-z plane. Right: Configuration of the silicon detector in $\mathrm{r}-\phi$ plane.

superlayer consists of 168 cells and the outermost one consists of 480 cells. Each cell contains twelve sense wires and thirteen potential wires placed alternately. The chambers are filled with a mixture of argon and ethane gasses with an admixture of $1.7 \%$ isopropanol and $\sim 100 \mathrm{ppm}$ oxgen. The main mixture is chosen to have a uniform drift velocity across the cell volume and admixture is for preventing the aging.

Along a charged particle passing through the chamber, the gasses in the chamber are ionized. The electrons are drifted toward the sense wire by the electric field, and then they create an avalanche of charges which induce a pulse onto the sense wire. The position resolution of the COT is about $140 \mu \mathrm{m}$ per cell, and the transverse momentum resolution is $\frac{\sigma_{p_{T}}}{p_{T}^{2}}=0.0015[\mathrm{GeV} / c]^{-1}$.

\section{Time-of-Flight System (TOF)}

The TOF detector [21] is incorporated into the CDF detector in order to identify particles up to $1.5 \mathrm{GeV} / c$. By measuring the time it takes for a collision product to reach the TOF, we can separate particles which have different masses, such as $\pi^{ \pm}$and $K^{ \pm}$. This detector is located between the COT and the superconducting solenoid at a radius of $140 \mathrm{~cm}$ with a coverage in $|\eta| \leq 1.0$. In this analysis, we do not use for particles discrimination but use for the event veto coming from cosmic rays.

\section{Superconducting Solenoid}

The superconducting solenoid operated at a current of about 4650 A produces an uniform magnetic field of $1.4 \mathrm{~T}$ parallel to the $z$-axis. The conductor is made of Al-stabilized NbTi. This strong magnetic field bends the trajectory of high- $p_{T}$ charged particles, allowing us to reconstruct their momentum using the tracking system. 


\subsubsection{Calorimeters}

The CDF calorimeters measure the energy of both charged and neutral particles. They are sampling scintillator calorimeters segmented into towers having a geometry projected to the detector center. The calorimeter system consists of electromagnetic (EM) and hadronic calorimeters, covering $2 \pi$ in azimuth over the range $|\eta|<3.6$. The cross section of the CDF calorimeter system is shown in Figure 2.5.

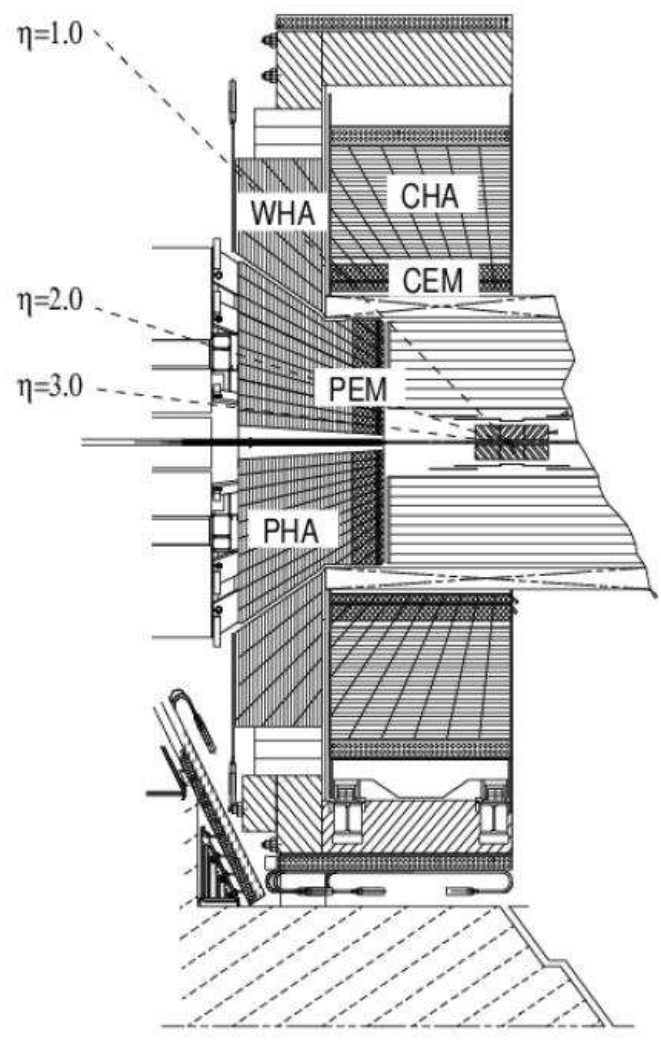

Figure 2.5: The CDF calorimeter system.

The EM calorimeter system consists of two sections: the central EM calorimeter [22] $(|\eta|<$ 1.1) and the plug EM calorimeter [23] $(1.1<|\eta|<3.6)$. Both sections include the main calorimeter, which mainly measures the energy of particles, and the shower maximum detector, which helps to improve the position resolution of the calorimeter clusters. The hadronic calorimeter system consists of three sections: the central hadronic calorimeter $(|\eta|<0.9)$, the wall hadronic calorimeter $(0.7<|\eta|<1.3)$, and the plug hadronic calorimeter $(1.3<|\eta|<3.6)$.

\section{Central Electromagnetic Calorimeter (CEM)}

The CEM is segmented into 24 towers in $\phi$ and 10 towers in $\eta$ on each side. The single CEM wedge is shown in Figure 2.6. It is a lead-scintillator sampling calorimeter having a radiation length $\left(X_{0}\right) 18 X_{0}$. The energy resolution of the CEM is

$$
\frac{\sigma_{E}}{E}=\frac{13.5 \%}{\sqrt{E(\mathrm{GeV})}} \oplus 2 \%
$$


where the notation $\oplus$ sums the constant and stochastic term in quadrature.

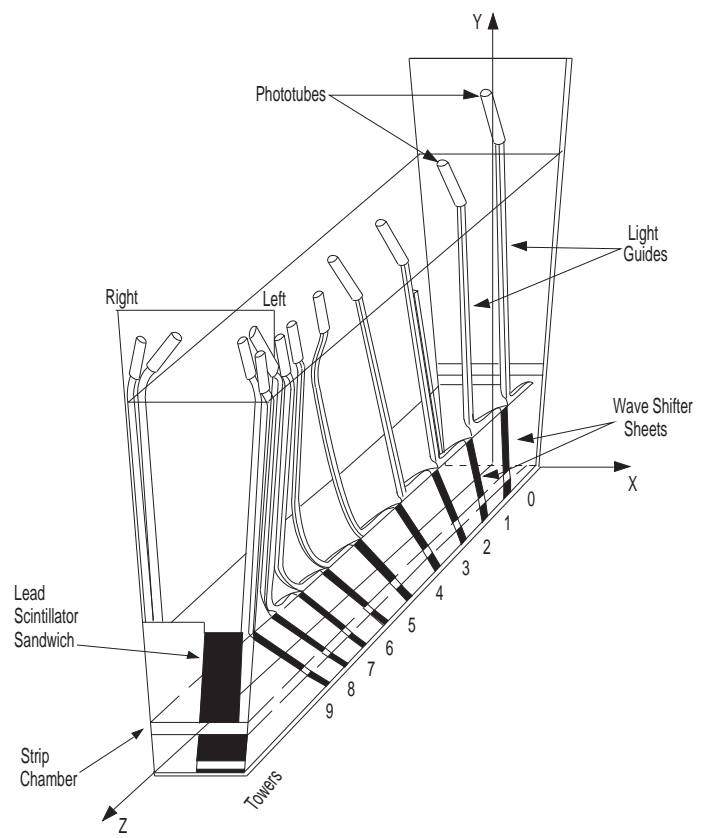

Figure 2.6: The single CEM wedge.

\section{Central Electromagnetic Shower Maximum Detector (CES)}

The CES is a proportional chamber with wire and strip readout which improves the position resolution of the shower clusters. The CES is placed near at a position at which the shower for electrons and photons has the maximum number of particles, called the shower maximum. Its position corresponds to a depth of $6 X_{0}$ of the EM calorimeter. The position resolution is $0.2 \mathrm{~cm}$ at $50 \mathrm{GeV}$.

\section{Plug Electromagnetic Calorimeter (PEM)}

The PEM is segmented into 12 towers in $\eta$ and 24 (48) towers in $\phi$ for the inner (outer) groups. It is a lead-scintillator sampling calorimeter having a total thickness is $21 X_{0}$. The energy resolution of the PEM is:

$$
\frac{\sigma_{E}}{E}=\frac{16.0 \%}{\sqrt{E(\mathrm{GeV})}} \oplus 1 \%
$$

\section{Plug Electromagnetic Shower Maximum Detector (PES)}

The PES [24] measures the shower maximum position similar to the CES. It is located at $6 X_{0}$ depth and is made of two layers of $5 \mathrm{~mm}$ wide scintillator strips, with each layer having a $45^{\circ}$ crossing angle relative to the other. 


\section{Central Hadronic Calorimeter (CHA)}

The CHA, iron-scintillator sampling calorimeters, is segmented into 24 towers in $\phi$ and 8 towers in $\eta$ [25]. It is located directly outside of the CEM with 32 layers per tower, which corresponds to 4.7 interaction lengths $\left(\lambda_{I}\right)$ thick. The energy resolution of the CHA is:

$$
\frac{\sigma_{E}}{E}=\frac{50 \%}{\sqrt{E(\mathrm{GeV})}} \oplus 3 \%
$$

for single $\pi^{ \pm}$that do not interact in the CEM.

\section{Wall Hadronic Calorimeter (WHA)}

The WHA extends the CHA coverage to fill the gap between the central and plug regions [25]. It is made of 15 layers of iron $(5.0 \mathrm{~cm})$ and scintillator $(1.0 \mathrm{~cm})$. The energy resolution of the WHA is:

$$
\frac{\sigma_{E}}{E}=\frac{75 \%}{\sqrt{E(\mathrm{GeV})}} \oplus 4 \%
$$

for single $\pi^{ \pm}$that do not interact in the CEM.

\section{Plug Hadronic Calorimeter (PHA)}

The PHA is made of 23 layers of alternating iron and scintillator. The energy resolution of the PHA is:

$$
\frac{\sigma_{E}}{E}=\frac{80 \%}{\sqrt{E(\mathrm{GeV})}} \oplus 5 \%
$$

for single $\pi^{ \pm}$that do not interact in the PEM.

\subsubsection{Muon Detectors}

The energy of high- $p_{T}$ muons are not measurable with the calorimeter, since they pass through the detector materials by depositing only minimum ionizing energy. The CDF muon detectors consist of four systems and are located outside of the calorimeters. The muon system coverage is shown in Figure 2.7. A cross section view of a muon chamber is shown in Figure 2.8. When a muon passes through the muon system, the drift time in each layer is registered. We define a muon "stub" which requires a hit in three of the four layers of drift chambers.

\section{Central Muon Detector (CMU)}

The CMU [26] consists of four layers of planar drift chambers located outside of the CHA. It covers the central region of $|\eta|<0.6$ and can detect muons with $p_{T}>1.4 \mathrm{GeV} / c$.

\section{Central Muon Upgrade (CMP)}

The CMP consists of four layers of planar drift chambers located outermost of the CDF for walls or behind the magnet return yokes. The CMP covers $|\eta|<0.6$ and can detect muons with $p_{T}>$ $2.0 \mathrm{GeV} / c$. 


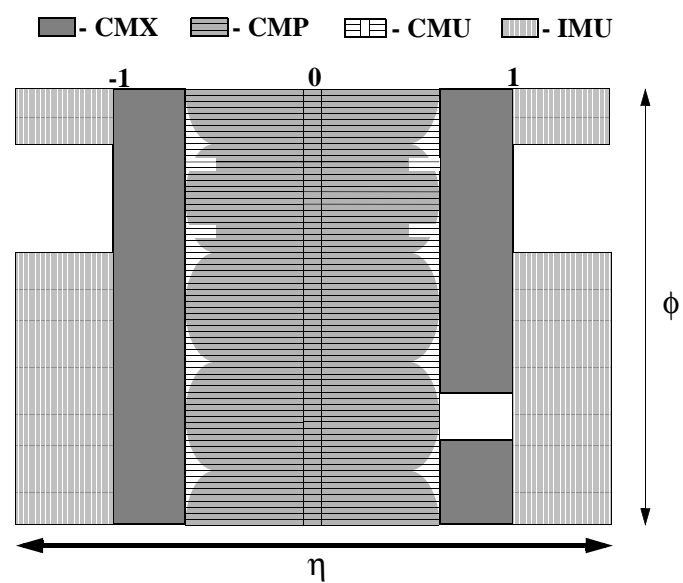

Figure 2.7: CDF muon coverage for the CMU, CMP, CMX, and BMU detectors. The BMU is referred as the IMU.

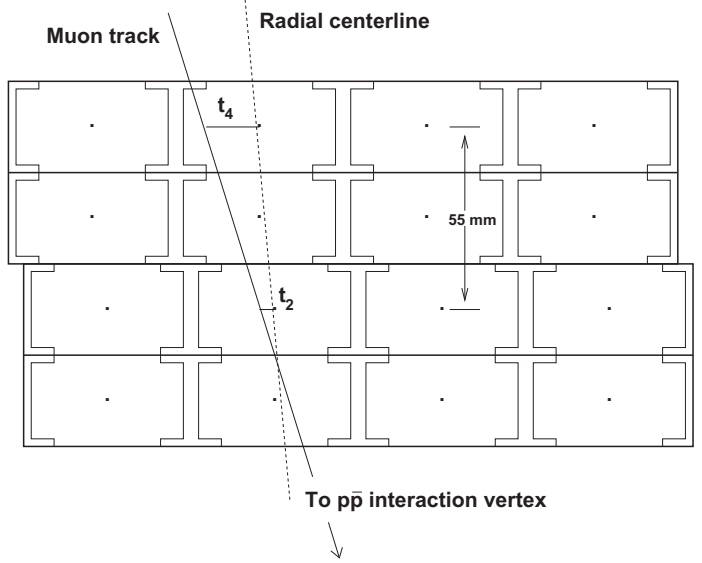

Figure 2.8: A cross section view of a CMU muon chamber.

\section{Central Muon Extension (CMX)}

The CMX consists of four to eight layers of drift chambers depending on the polar angle. It provides muon detection in the region $0.6<|\eta|<1.0$ and can detect muons with $p_{T}>1.4 \mathrm{GeV} / c$. The scintillator tiles (CSX) is also placed on the inside and outside of the CMX, used for improved triggering.

\section{Barrel Muon Detector (BMU)}

The BMU extends the muon detector coverage to $1.0<|\eta|<1.5$. Drift chambers and scintillators are attached surrounding the forward toroid magnets. 


\subsubsection{Luminosity Monitor}

\section{Luminosity}

The collision rate of protons and antiprotons is quantified by the instantaneous luminosity. The luminosity is calculated with the following formula:

$$
\mathcal{L}=\frac{f_{r} N_{B} N_{p} N_{\bar{p}}}{2 \pi\left(\sigma_{p}^{2}+\sigma_{\bar{p}}^{2}\right)} F\left(\frac{\sigma_{l}}{\beta^{*}}\right)
$$

where $f_{r}$ is the revolution frequency, $N_{B}$ is the number of bunches, $N_{p}$ and $N_{\bar{p}}$ are the number of protons or antiprotons per bunch, $\sigma_{p}$ and $\sigma_{\bar{p}}$ are the beam sizes at the interaction point, $F$ is a form factor which corrects for the bunch shape and depends on the ratio of the bunch length $\sigma_{l}$ to the beta function $\beta^{*}$ at the interaction point. $\beta^{*}$ is a measure of the beam spreads, which are given by $\sqrt{\beta^{*} \epsilon}$ with $\epsilon$ being the beam emittance.

There is a continuous effort to maximize the peak luminosity which directly results in increasing the amount of data delivered by the Tevatron. The amount of data collected through Run II is expressed by the integrated luminosity $\left(\int \mathcal{L} \mathrm{dt}\right.$ ) which is measured in units of $\mathrm{b}^{-1}$, where $1 \mathrm{~b}^{-1}$ is $10^{24} \mathrm{~cm}^{-2}$. Figure 2.9 shows the integrated luminosity delivered by the Tevatron and recorded by the CDF.

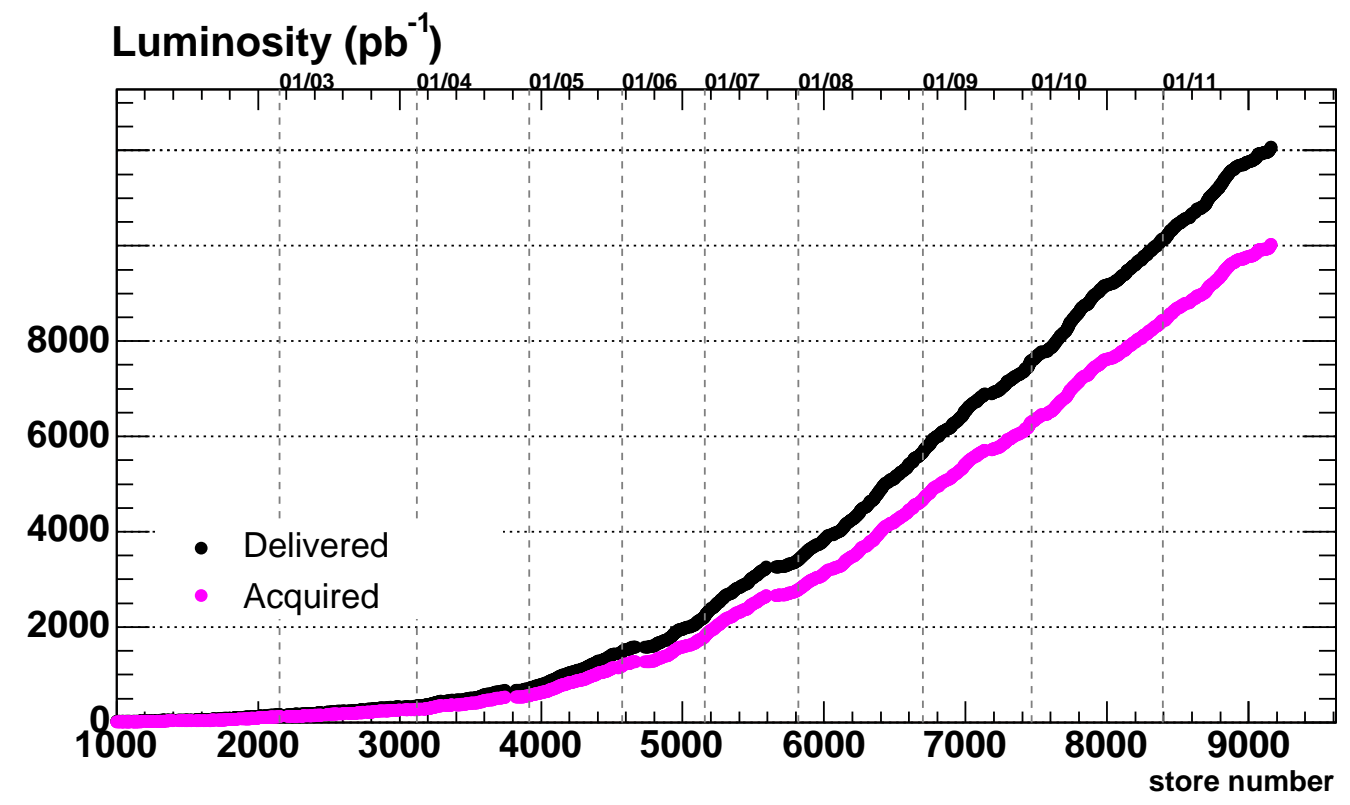

Figure 2.9: Total integrated luminosity delivered by the Tevatron and recorded by the CDF.

\section{Cherenkov Luminosity Counters (CLC)}

The beam luminosity is measured by using the CLC detector [27] located in the forward region $(3.75<|\eta|<4.75)$ of the CDF detector on both sides. The CLC consists of long conical gaseous Cherenkov counters that monitor the average rate of inelastic $p \bar{p}$ collisions per bunch crossing $\left(R_{p \bar{p}}\right)$. The instantaneous luminosity $\left(\mathcal{L}_{i n s t}\right)$ is calculated from the next expression:

$$
\mu \cdot f_{B C}=\sigma_{i} \cdot \mathcal{L}_{i n s t} \cdot \epsilon
$$


where $\mu$ is the average number of $p \bar{p}$ interactions per bunch crossing, $f_{B C}$ is the bunch crossing frequency $(2.5 \mathrm{MHz}$ for $36 \times 36$ bunch operations), $\epsilon$ is the CLC acceptance for a single $p \bar{p}$ interaction, and $\sigma_{i}$ is the inelastic $p \bar{p}$ cross section at the Tevatron $(60.7 \pm 2.4 \mathrm{mb})$.

\subsubsection{Data Acquisition System}

The bunch separation of the Tevatron is $396 \mathrm{~ns}$ for $36 \times 36$ bunch operations which corresponds to $2.5 \mathrm{MHz}$. Since this rate is too high to record every event into disk, we need to discard the most events while interesting ones must be identified. The selection of events is performed by the fast online electronics, called the trigger system. The CDF trigger system has a three level architecture (called Level 1, Level 2, and Level 3) and is designed to reduce the data rate by identifying the physically interesting events. Once an event is accepted by the Level 3 trigger, then the data are sent to the Consumer Sever/Logger (CSL) that is the final component in the CDF data acquisition system. The data flow in the CDF trigger system is shown in Figure 2.10.

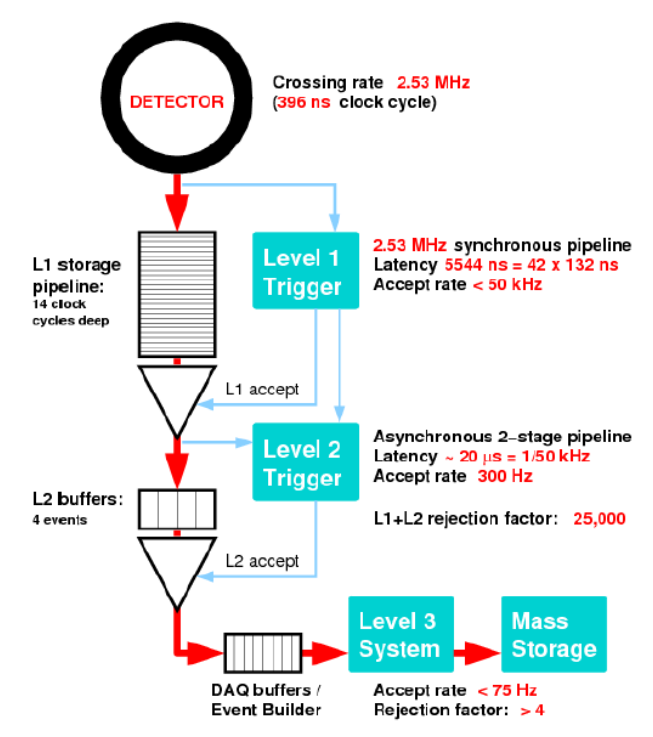

Figure 2.10: Data flow in the CDF data acquisition system.

\section{Level 1}

The Level 1 trigger (L1) acceptace rate is less than $50 \mathrm{kHz}$. Figure 2.11 shows the trigger path from the Level 1 to the Level 2. The L1 CAL can make its decision based on clusters of energy in the calorimeters, missing energy from the energy conservation, or the sum of calorimeter energy. A system called Extremely Fast Tracker (XFT) [28] reconstructs tracks using the COT information, and the tracks found by the XFT are used for the L1 trigger decision. The CMU can also provide L1 trigger for muon candidates.

\section{Level 2}

The Level 2 trigger (L2) reduces the event rate to $300 \mathrm{~Hz}$. The L2 system consists of several asynchronous subsystems which provide input to programmable L2 processors. The L2 decisions are made based on the following: 


\section{RUN II TRIGGER SYSTEM}

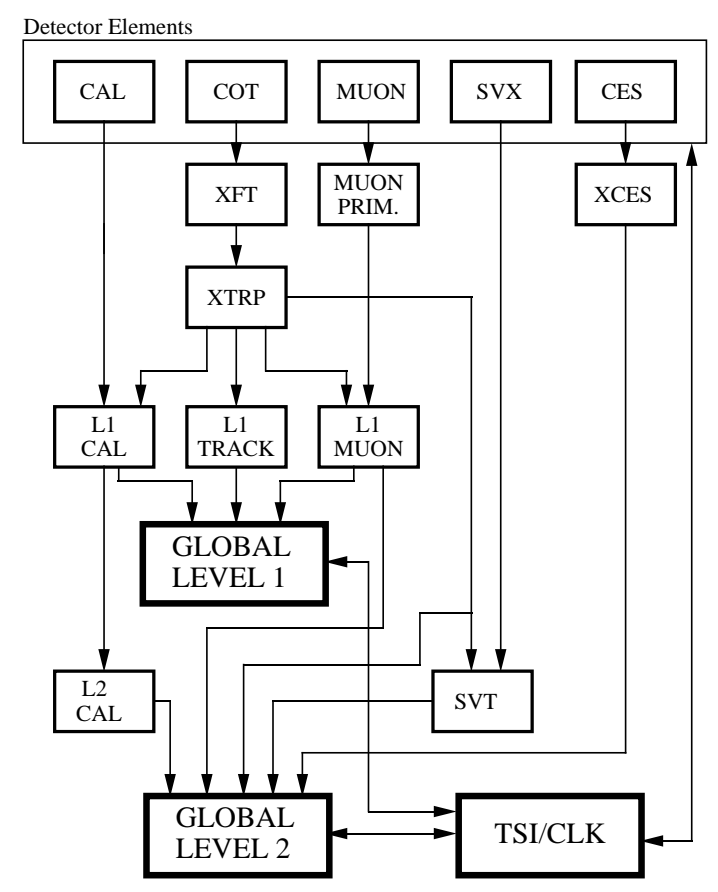

PJW 9/23/96

Figure 2.11: Block diagram of the Level 1 and Level 2 trigger path.

- L2 cluster finder (L2CAL): The L2CAL combines adjacent calorimeter towers over $1 \mathrm{GeV}$ threshold starting from a seed tower of minimum $3 \mathrm{GeV}$.

- CES information: The shower maximum detector information provides the position resolution for electron and photon showers with better than the cluster location. It is also used to match with the tracking information.

- Silicon Vertex Tracker (SVT) [29]: The SVXII information is combined with the L1 XFT track information by the SVT, a system that rapidly analyzes the silicon data to look for a displaced vertex.

- Muon information: The muon trigger combines information from the muon detector and from the L1 XFT track.

\section{Level 3}

The Level 3 trigger (L3) consists of a few hundred of computers. The L3 computer farm reconstructs the event in software and filter the event rate to about $75 \mathrm{~Hz}$. Events that pass L3 decision are written to disk. 


\section{Consumer Server/Logger}

Once an event is accepted by the L3 trigger, it sends to the Consumer Server/Logger (CSL) system. The CSL is responsible for categorizing events by the trigger path, writing them to the disk, and sending a fraction of events to online processors for real time monitoring of data quality. 



\section{Chapter 3}

\section{Event Reconstruction}

We search for the Higgs boson in $g g \rightarrow H \rightarrow W W \rightarrow \ell \nu j j$, which includes a lepton, missing energy, and two jets as the final observables. Definition and description of the relevant observables for high level detector objects and the event reconstruction are discussed in this chapter.

\subsection{High Level Detector Object}

The detector raw outputs are primarily used to reconstruct high level detector object, which includes tracks, sequences of hits left by charged particles as they pass through the tracking detectors, and calorimeter clusters, collections of towers in which energy from particles is deposited. Associating these together, with applying quality cuts, we reconstruct electrons, muons, jets and missing transverse energy.

\subsubsection{Tracking}

The tracking system of the CDF II detector is described in Section 2.2.1. It detects trajectories of charged particles. This information is used in primary vertex reconstruction, lepton identification, and heavy flavor tagging.

The tracking detectors are enclosed by the superconducting solenoid, which provides a uniform magnetic field inside the detector with field lines parallel to the beam-axis. Charged particles traveling through magnetic field are curved depending on their momenta and charges. The resulting trajectory in transverse plain is a helix. The following parameters are used in the description of the trajectory in three dimensions:

$C:$ half-curvature of the trajectory defined as

$$
C=\frac{1}{2 Q \rho}
$$

where $\rho$ is the radius of the projection of the trajectory to the transverse plane. It carries the same sign as the charge of the particle and is inversely proportional to the $p_{T}$ of the track.

$\cot \theta$ : cotangent of the polar angle of the trajectory at closest approach to the origin.

$\boldsymbol{d}_{0}$ : the impact parameter, the minimal distance between the trajectory and the origin in the transverse plane, defined as

$$
d_{0}=Q \cdot\left(\sqrt{x_{0}^{2}+y_{0}^{2}}-\rho\right)
$$

where $x_{0}$ and $y_{0}$ are the coordinates of the center of the helix circle in the transverse plane. 
$\phi_{0}$ : the azimuthal angle of the trajectory at the point of closest approach to the detector center.

$z_{0}:$ the $z$ position of the trajectory point closest to the detector center.

We use four tracking algorithms in this analysis, called COT stand-alone tracking, Outside-In (OI) tracking, Inside-Out (IO) tracking, and Phoenix tracking.

The primary tracking algorithm of CDF begins with the COT. Firstly, hits in the COT are identified. These are associated together within each superlayer to form short track segments, which can be combined across superlayers to form a track. Because the superlayers of the COT alternate in orientation between axial and stereo superlayers, the tracking algorithm compares segments in all axial superlayers first, starting with tracks in the most outer superlayer and finding the segment that gives the best fit. It then adds the stereo superlayers and performs the fit again to create a final COT stand-alone track. COT stand-alone tracks are required to have hits in at least four of eight superlayers, using $\geq 2$ axial and $\geq 2$ stereo superlayers.

The OI tracking can extend the COT stand-alone tracks by adding high-resolution hit information from the silicon detector. It attaches axial silicon hits to COT tracks and then performs pattern recognition on the small angle and $90^{\circ}$ stereo strips. At each wafer intersected by the seed track, every hit is attached to a separate copy of the seed track. Then, the track is extrapolated to the next wafer where the same matching procedure is performed until the track reaches the most inner silicon wafer. At the end, the track combination with the highest number of hits and lowest $\chi^{2} /$ dof is kept.

Due to the limited COT coverage $(|\eta|<1.0)$ and the strict hit requirement (at least four of eight superlayer), tracking in the forward region requires other algorithms, called IO tracking and Phoenix tracking. IO tracking algorithm firstly finds tracks in the silicon detector, requiring hits in at least three layers, then extends them by adding hits in the COT that are not already used to form COT stand-alone or OI tracks. Phoenix tracking uses a cluster in the plug calorimeter and the primary vertex as two points of the track and then looks for hits in between that would complete the track. It uses the energy of the cluster to estimate the momentum of the particle, giving a curvature estimate which is used to search for hits in the tracking region.

In this analysis, most tracks are from a collection which includes COT stand-alone tracks, OI tracks, and IO tracks.

\subsubsection{Primary Vertex}

Primary vertex location is required to calculate transverse energies in the calorimeter towers and to derive objects such as jets and missing energy, since the angle $\theta$ is defined by the vector pointing from the vertex to the shower maximum detectors within the calorimeter towers. Using the detector center, instead of the $z$-position of the vertex in the $\mathbb{E}_{T}$ calculation, has an impact on the $\mathbb{E}_{T}$ beyond the level of the corrections applied for jets and muons.

Vertices are found by fitting good quality tracks [30]. At high luminosities, several collisions may occur at one bunch crossing. The position of the vertex in the $z$-coordinate has a Gaussian spread of $\sigma_{z}=29 \mathrm{~cm}$ around the detector center. Therefore, the vertices for multiple interactions are normally separated in $z$-position. The estimate of the vertices is started with finding seed vertices in the $z$-coordinate. Then, the $z$-position of each vertex is calculated from the weighted average of the $z$-coordinate of all tracks within $1 \mathrm{~cm}$ of the seed vertex. The primary vertex is determined by an iterative vertex finding algorithm which uses tracks around a seed vertex to form a new vertex position. The $\chi^{2}$ for all tracks relative to the new vertex is calculated, tracks with degraded $\chi^{2}$ are removed, and the cycle is repeated until all tracks have a good $\chi^{2}$. We finally 
define the vertex associated with the highest sum of the track $p_{T}$ as the primary vertex of the event.

\subsubsection{Calorimeter Clustering}

High momentum electrons, photons, and jets leave energy in small continuous groups of calorimeter towers which can be identified as an energy cluster. Firstly, calorimeter clustering algorithm starts finding a seed cluster that has an energy larger than a certain threshold. Then, adjacent towers with energy greater than a lower threshold are added to form a cluster. The position of the cluster is defined by the energy-weighted mean of the towers in the cluster, and the total energy is estimated by the sum of the energies of the towers in the cluster. Once a cluster is defined, the precision of its position can usually be improved by matching it with a cluster in the shower maximum detector, which is constructed with a similar algorithm but which is optimized to achieve higher position resolution.

\subsection{Particle Identification}

Once high level detector objects are reconstructed, it is possible to identify physical objects from which we start physics analysis. In this section, we describe the identification of leptons, neutrinos (missing transverse energy), and jets. These objects are the final state of our signal process $H \rightarrow$ $W W \rightarrow \ell \nu b \bar{b}$.

\subsubsection{Leptons}

\section{Electron}

This analysis considers central electrons(CEM).

The central electron reconstruction starts from a track with $p_{T}>9 \mathrm{GeV} / c$ that extrapolates to a cluster of CEM with $E_{T}>20 \mathrm{GeV}$. To improve the purity of the electron selection, we apply several cuts as summarized in Table 3.1. The variables in Table 3.1 are defined as:

- $p_{T}$ : The track $p_{T}$ associated to the EM cluster.

- $E_{T}$ : The transverse energy of the electron candidate defined as $E_{T}=E \sin \theta$.

- $E_{\mathrm{HAD}} / E_{\mathrm{EM}}$ : The ratio of the total hadronic cluster energy to the total EM energy.

- $E / p$ : The ratio between the cluster energy to the momentum of the associated track.

- Isolation: The ratio of the transverse energy surplus in a cone of radius $\Delta R<0.4$ around the cluster center to the transverse energy of the cluster.

- $L_{\mathrm{shr}}$ : The difference between the lateral distribution of the energy in the calorimeter towers and what is expected for an electromagnetic shower. The expectation is derived from simulations and modified to fit test beam data. This requirement helps remove hadronic showers that might imitate electromagnetic showers.

- $\left|\Delta_{x}\right|_{\text {CES }}$ and $\left|\Delta_{z}\right|_{\text {CES: }}$ The distances in the transverse plane and along the $z$-axis between the cluster position from the CES measurements and the extrapolated track associated with the cluster. 
Table 3.1: Selection requirement for central electrons [31].

\begin{tabular}{c}
\hline \hline Central Electron Selection \\
\hline To be in the active region of CES and CEM \\
$p_{T} \geq 10 \mathrm{GeV} / c$ \\
$E_{T} \geq 20 \mathrm{GeV}$ \\
COT Axial Segments $\geq 3$ \\
COT Stereo Segments $\geq 2$ \\
Hits per COT Segments $\geq 5$ \\
$\left|z_{0}\right|_{\text {track }} \leq 60 \mathrm{~cm}$ \\
$E_{\mathrm{HAD}} / E_{\mathrm{EM}} \leq 0.055+0.00045 \times E / \mathrm{GeV}$ \\
$E / p \leq 2.0$ unless $p_{T} \geq 50 \mathrm{GeV} / c$ \\
Isolation $\leq 0.1$ \\
$L_{\text {shr }} \leq 0.2$ \\
$\left|\Delta_{z}\right|_{\mathrm{CES}} \leq 3.0 \mathrm{~cm}$ \\
$-3.0 \leq Q \times\left.\Delta_{x}\right|_{\mathrm{CES}} \leq 1.5 \mathrm{~cm}$ \\
$\chi_{\mathrm{CES}}^{2} \leq 10$ \\
Photon Conversions Veto \\
\hline \hline
\end{tabular}

- $\chi_{\mathrm{CES}}^{2}$ : Comparison of the CES shower profile with that of the test beam electrons given as the chi-square probability for the fit.

- Photon conversion veto: Electrons from photon conversions through the detector material are vetoed by rejecting electron candidates if an oppositely charged track is found with a small distance of closest approach.

\section{Muon}

This analysis considers two types of muons: CMUP and CMX. These types are basically defined by the detectors which they pass through.

The central muon reconstruction is summarized in Table 3.2. Since CMU stand-alone muon candidates have a large fraction of fake muons coming from the punch-through particles through calorimeter, we also require a CMP detector stub to suppress fake muons and call such muons as CMUP muons. The range $0.65<|\eta|<1.0$ is covered by CMX detector. The selection requirements are explained below:

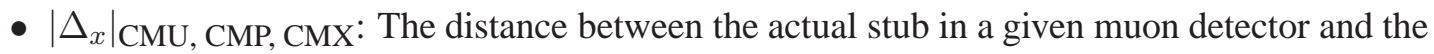
track position extrapolated to that detector.

- Cosmic Rays Veto: The impact parameter $d_{0}$ of the tracks are used to kill cosmic rays. Because most cosmic rays pass through the detector from the top to bottom, they often look back-to-back tracks reconstructed $d_{0}$ being identical.

\subsubsection{Jets}

Jets are the results of hadronization of quarks and gluons. In this analysis, we reconstruct jets from a group of electromagnetic and hadronic calorimeter clusters which fall within a cone of radius 
Table 3.2: Selection requirement for central muons [31].

\begin{tabular}{c}
\hline \hline Central Muon Selection \\
$p_{T} \geq 20 \mathrm{GeV} / c$ \\
COT Axial Segments $\geq 3$ \\
COT Stereo Segments $\geq 2$ \\
Hits per COT Segments $\geq 5$ \\
$\left|d_{0}\right|_{\text {track }} \leq 0.2 \mathrm{~cm}$ if no silicon hits \\
$\left|d_{0}\right|_{\text {track }} \leq 0.02 \mathrm{~cm}$ if silicon hits \\
$E_{\mathrm{EM}} \leq \max (2,2+0.0115(p-100)) \mathrm{GeV}$ \\
$E_{\mathrm{HAD}} \leq \max (6,6+0.0280(p-100)) \mathrm{GeV}$ \\
$\left|\Delta_{x}\right|_{\mathrm{CMU}} \leq 3.0 \mathrm{~cm}$ \\
$\left|\Delta_{x}\right|_{\mathrm{CMP}} \leq 5.0 \mathrm{~cm}$ \\
$\left|\Delta_{x}\right|_{\mathrm{CMX}} \leq 6.0 \mathrm{~cm}$ \\
Isolation $\leq 0.1$ \\
Cosmic Rays Veto \\
\hline \hline
\end{tabular}

$\Delta R=\sqrt{(\Delta \phi)^{2}+(\Delta \eta)^{2}} \leq 0.4$ [32]. The calorimeter clusters which belong to electron candidate are not taken account for the jet reconstruction. Since the raw energy does not predict the correct parton energy due to instrumental and physical reason, we apply several jet energy corrections to estimate the energy of parton [33]. The corrected jet energy is expressed in the following equation:

$$
E_{T}^{\text {parton }}=\left(E_{T, \text { jet }}^{\text {measured }} \times f_{\text {rel }}-E_{T}^{\mathrm{MI}} \times N_{\mathrm{vtx}}\right) \times f_{\mathrm{abs}}-E_{T}^{\mathrm{UE}}+E_{T}^{\mathrm{OOC}}
$$

where correction factors are:

$f_{\text {rel }}:$ a scale factor to make the jet response uniform over the pseudo-rapidity

$E_{T}^{\mathrm{MI}} \times N_{\mathrm{vtx}}:$ a correction for multiple interactions per beam crossing

$f_{\text {abs }}$ : an absolute energy correction determined by matching parton energy with jet energy

$\boldsymbol{E}_{\boldsymbol{T}}^{\mathrm{UE}}$ : the contribution coming from the underling events

$E_{T}^{\mathbf{O O C}}$ : an out-of-cone correction which is the energy of the parton emitted outside the jet cone

\subsubsection{Missing Transverse Energy}

Neutrinos cannot be detected by the detector, but they are identified from the missing energy. Since the $z$-component of momentum of the interacting partons is unknown, we cannot determine the $z$-component of the neutrino energy. However, we can determine the components in the transverse plane since the transverse momentum sum of initial partons can be ignored. The uncorrected missing transverse energy $\mathscr{E}_{T}^{\mathrm{raw}}$ is the negative of the vector sum of all calorimeter tower depositions projected on the transverse plane:

$$
{\overrightarrow{E_{T}}}^{\text {raw }}=-\sum_{i=\text { towers }}{\overrightarrow{E_{T}}}^{i}, \ddot{E}_{T}^{\text {raw }}=\left|{\overrightarrow{E_{T}}}^{\text {raw }}\right|
$$


Since this missing transverse energy is measured from the raw calorimeter response, it needs to be corrected for the jet energy correction and for the muons escaped from the calorimeter. The missing transverse energy $\mathbb{E}_{T}$ used in this analysis is then

$$
E_{T}=E_{T}^{\mathrm{raw}}-\sum_{\text {muon }} p_{T}+\sum_{\text {muon }} E_{T}(\mathrm{EM}+\mathrm{Had})-\sum_{j e t} E_{T}(\text { Jet Energy Correction })
$$

\subsubsection{Neutrino $p_{z}^{\nu}$ Reconstruction}

Since there is a neutrino in the final state $H \rightarrow W W^{(*)} \rightarrow l \nu j j$, there remains an uncertainty as to the z-component of the neutrino momentum.

We simply asuume that the leptonic decaying $W$ to be on-shell, and solve $p_{z}^{\nu}$ with this equation:

$$
m(l, \nu)=80.419 \mathrm{GeV} / \mathrm{c}^{2}
$$

[34]. This is a quadratic equation, and gives two real solutions in some events, and two imaginary solutions in others. In the case where we have imaginary solutions, we simply pick up the real part of the solution. When we have two real solutions, choose the solution that has a smaller absolute value since this turned out to be more probable.

Figure 3.1 shows the performance of this neutrino $p_{z}^{\nu}$ reconstruction. The black line shows all the events. The "imaginary" histogram shows the events where the on-shell $W$ mass assumption ended up in imaginary $p_{z}^{\nu}$ solutions. The "correct" histogram shows the events where we successfully picked up the $p_{z}^{\nu}$ solution that is closer by value to the HEPG information than the other solution. Note that the "imaginary" events are considered to be "correct" here.

The reconstructed mass peaks around the Higgs mass input to the Monte Carlo generation, showing the present mass reconstruction method is applicable.

The reconstructed Higgs mass, however, tends to have a tail to the higher mass, even in the correctly reconstructed events. To understand this tendency, we plot in Figure 3.2 the reconstructed Higgs mass for events where the analyzed two jets match the two partons coming from a $W$ boson from the Higgs decay. The distribution is much narrower than in Figure 3.1, suggesting that the tail in the distribution is due to the events where we pick up jets that are not come from the Higgs decay.

Since the peak seems not affected much by the reconstruction, we fit the reconstructed Higgs mass to Gaussian in Figure 3.3. The $1 \sigma$ region around the peak is fit into gaussian.

Table 3.3 summarizes the "imaginary" and "correct" fractions as well as the Higgs mass resolution for different Higgs mass assumptions. Note that the "correct" fraction is inclusive of the "imaginary" fraction. To be more clear, $100-31.1=68.9 \%$ of the events give real solutions, and we pick up the correct real solution in $79.5-31.1=48.4 \%$ of the entire events. Therefore, we pick up the correct solutions in $70.2 \%$ of the cases where the solution to real. 

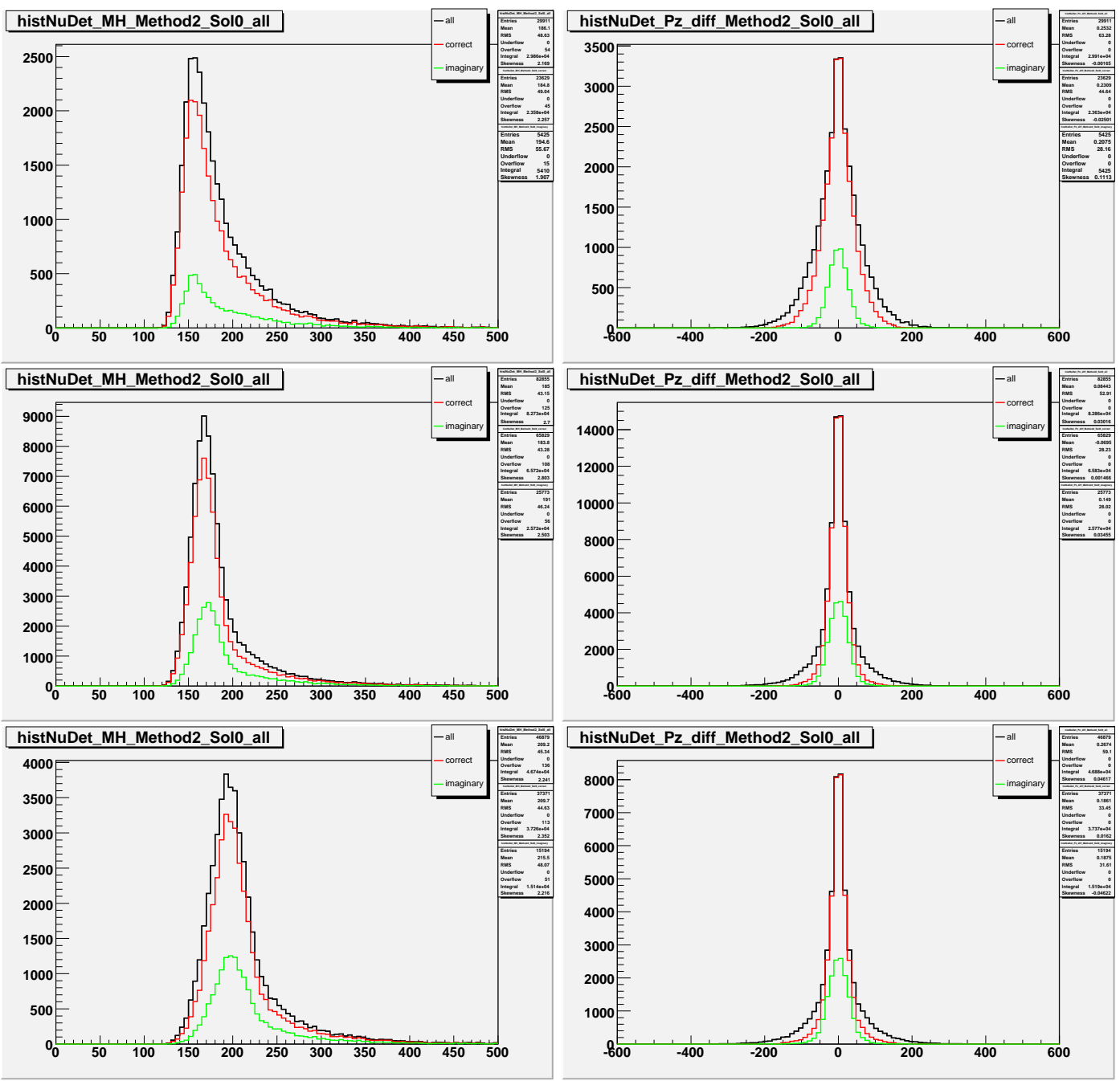

Figure 3.1: Performance of $p_{z}^{\nu}$ reconstruction. Top, middle, and bottom rows correspond to signal Monte Carlo generated with $m_{H}=150,170$ and $200 \mathrm{GeV} / \mathrm{c}^{2}$. The left plots show the reconstructed Higgs mass, and the right show the reconstructed $p_{z}^{\nu}$ value subtracted by the truth (in HEPG). See text for the definition of the "correct" and "imaginary" histograms.
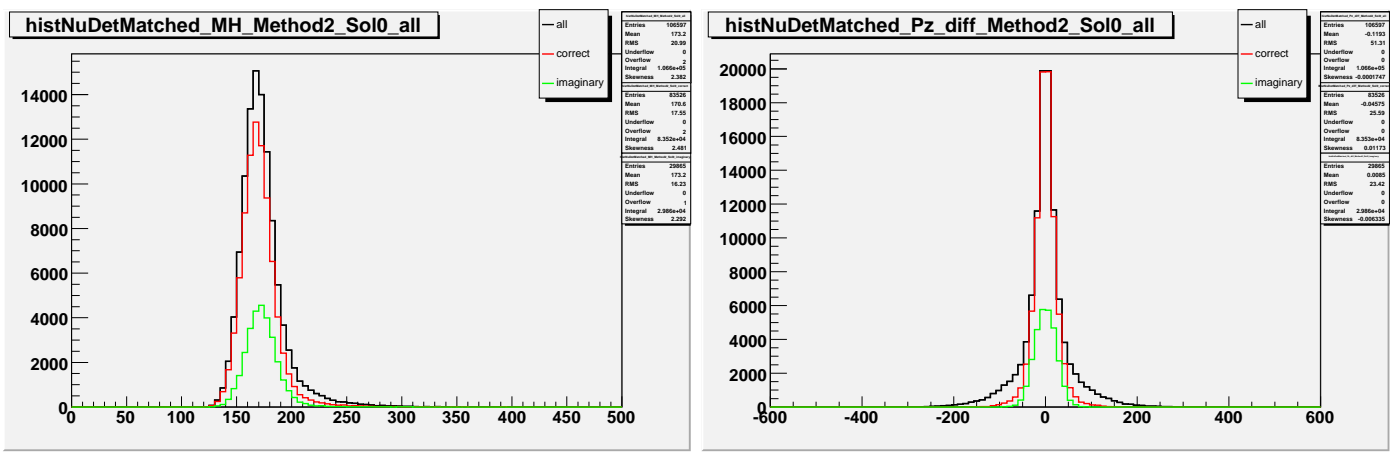

Figure 3.2: Performance of $p_{z}^{\nu}$ reconstruction for events where the two analyzed jets match the HEPG partons that come from the decay of the Higgs boson, for $m_{H}=170 \mathrm{GeV} / \mathrm{c}^{2}$. The left plot shows the reconstructed Higgs mass, and the right shows the reconstructed $p_{z}^{\nu}$ value subtracted by the truth (in HEPG). See text for the definition of the "correct" and "imaginary" histograms. 

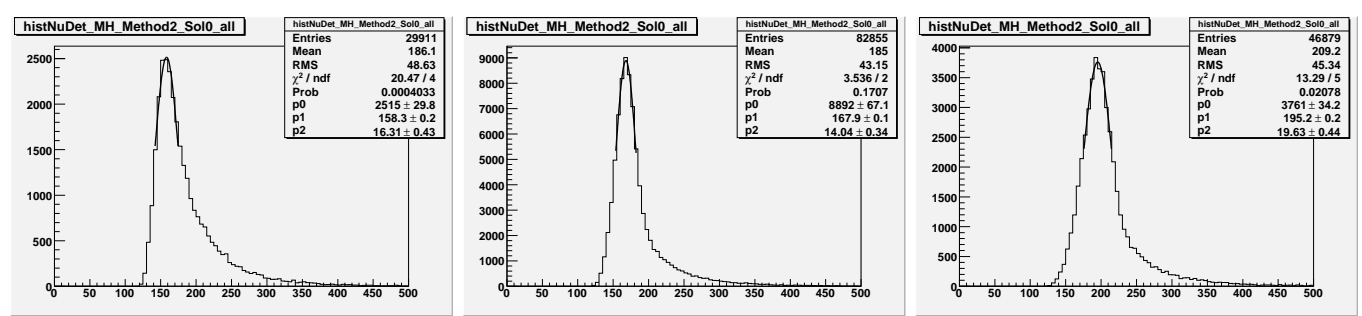

Figure 3.3: Fit of the reconstructed Higgs mass to a Gaussian. Left, center and right plots show signal Monte Carlo for $m_{H}=150,170,200 \mathrm{GeV} / \mathrm{c}^{2}$ respectively.

Table 3.3: Fraction of events with imaginary solutions, and of events where we pick up the correct neutrino $P_{\mathrm{Z}}$ solution. The resolution and mass for the reconstructed Higgs mass.

\begin{tabular}{ccccc}
\hline \hline input $m_{H}$ & imaginary solution & correct solution & mass resolution $(\mathrm{GeV})$ & mass $(\mathrm{GeV})$ \\
\hline 150 & $18.1 \%$ & $79.0 \%$ & 16.3 & 158 \\
170 & $31.1 \%$ & $79.5 \%$ & 14.0 & 168 \\
200 & $32.4 \%$ & $79.7 \%$ & 19.6 & 195 \\
\hline \hline
\end{tabular}




\subsubsection{Distinction of Up- and Down-type Quark Jets}

The Higgs boson has a spin 0, and is expected to decay isotropically. This information is useful in reducing background, because the dominant background processes have different spins.

Plotted in Figure 3.4 are the lepton angle in the leptoniccally decaying $W$ 's rest frame against the up-type quark angle in the hadroniccally decaying $W$ 's rest frame. The plots are for the singal and dominant background processes. The angles are calculated using the HEPG information. One can clearly see that the signal has a quite different distribution than the background processes. Although we do not use these 2D plots in our analysis but rather include a 1D variable $(\Delta R$ between lepton and the down-type quark) into our likelihood discriminant, Figure 3.4 illustrates the impact of such spin information as the singal/background discriminant.
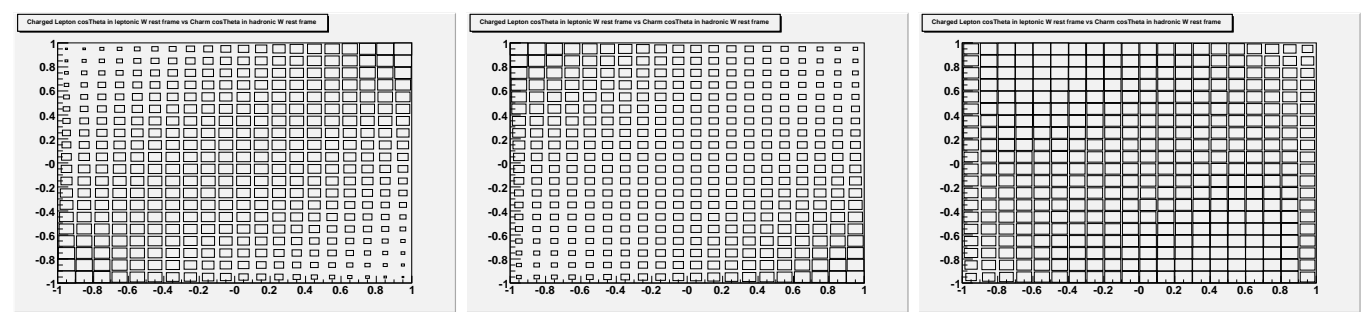

Figure 3.4: $\cos \theta_{l}^{*}$ against $\cos \theta_{u p-t y p e}^{*}$. From left: signal MC for $m_{H}=170 \mathrm{GeV} / \mathrm{c}^{2}$, electroweak $W W$ process, and $W+c+1$ parton.

In order to use such spin information in the analysis, it is crucial to distinguish between jets originating from up-type and down-type quarks in the hadronic $W$ decay.

We take the jet that is away from the lepton than the other jet as the up-type jet. Figure 3.5 shows HEPG level $\Delta \phi$ distribution between the lepton and the up-type quark and down-type quark in the Higgs decay.

We performed a study on matching the reconstucted jets with the hard process partons in the signal Monte Carlo sample $\left(m_{H}=170 \mathrm{GeV} / \mathrm{c}^{2}\right)$, in order to understand the validity of this reconstruction method. The two jets are matched to the quarks in the Higgs decay final state in $66.7 \%$ of the events. We found $88.4 \%$ of such events were reconstructed correctly with our up-/down-type distinction.

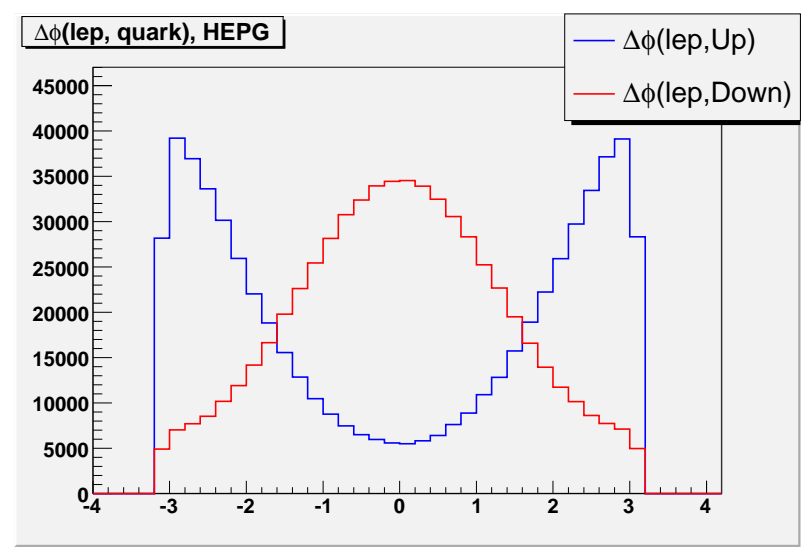

Figure 3.5: Blue(Red) histogram shows $\Delta \phi$ distribution between lepton and the up-type(downtype) jet in the Higgs decay. For signal Monte Carlo for $m_{H}=170 \mathrm{GeV} / \mathrm{c}^{2}$. 



\section{Chapter 4}

\section{Event Selection}

Our target physics process is $g g \rightarrow H \rightarrow W W^{(*)} \rightarrow l \nu j j$, and we require the four final state particles to be reconstructed with the $\mathrm{CDF}$ detector.

\subsection{Trigger Requirements}

Events considered in this analysis must first pass one of three high $p_{T}$ lepton trigger paths. The $\mathrm{CDF}$ trigger system is described in Section 2.2.5. The three trigger paths used in this analysis are ELECTRON_CENTRAL_18, MUON_CMUP18, MUON_CMX18. These central leptons are specifically designed to trigger on high- $p_{T}$ central electrons and muons using a minimal set of identification cuts. The specific trigger requirements are given in the following sub-sections.

\subsubsection{ELECTRON_CENTRAL_18}

The ELECTRON_CENTRAL_18 trigger path is designed to accept events which contain a high$p_{T}$ electron which enters the CEM. This trigger path consists of the following level 1, 2, and 3 requirements:

- L1_CEM8_PT8 requires a central EM cluster with a measured $E_{T}$ of at least $8 \mathrm{GeV}$, the ratio $E_{\mathrm{Had}} / E_{\mathrm{EM}}$ to be less than 0.125 , and an XFT track with $p_{T}>8.34 \mathrm{GeV} / c$.

- L2_CEM16_PT8 additionally requires an EM cluster with an $E_{T}$ of at least $16 \mathrm{GeV}$ in the range $|\eta|<1.317$.

- L3_ELECTRON_CENTRAL_18 requires an $L_{\text {shr }}$ less than 0.4 , the $\Delta_{z}$ between the COT track extrapolation and the CES shower location measurement to be less than $8 \mathrm{~cm}, E_{T}>$ $18 \mathrm{GeV}$, and a COT track with $p_{T}>9 \mathrm{GeV} / c$.

\subsubsection{MUON_CMUP18}

The MUON_CMUP18 trigger path is designed to accept events that contain a high- $p_{T}$ muon where a high-momentum track is associated with hits in both the CMU and CMP detectors. This trigger consists of the following level 1, 2, and 3 requirements:

- L1_CMUP6_PT4 requires an XFT track with $p_{T}>4.09 \mathrm{GeV} / c$ pointing to the CMP fiducial region and a CMU stub with a track having $p_{T}>6 \mathrm{GeV} / c$. 
- L2_CMUP6_PT8 additionally requires a 4-layer XFT track with $p_{T}>8.34 \mathrm{GeV} / c$ which is fiducial to both the CMU and CMP detectors.

- L3_MUON_CMUP18 requires a COT track with $p_{T}>18 \mathrm{GeV} / c$ whose extrapolation matches hits in the CMU and CMP detectors within $\Delta_{x C M P}<20 \mathrm{~cm}$ and $\Delta_{x C M U}<10$ $\mathrm{cm}$.

\subsubsection{MUON_CMX18}

The MUON_CMX18 trigger path is designed to accept events that contain a high- $p_{T}$ muon with a high-momentum track pointing to hits in the CMX detector. This trigger consists of the following level 1,2, and 3 requirements:

- L1_CMX6_PT8_CSX requires an XFT track with $p_{T}>8.34 \mathrm{GeV} / c$ pointing to the CMX fiducial region, a CMX stub with a track having $p_{T}>6 \mathrm{GeV} / c$, and a hit in the CSX.

- L2_CMX6_PT10 additionally requires a 4-layer XFT track with $p_{T}>10.1 \mathrm{GeV} / c$.

- L3_MUON_CMX18 requires a COT track with $p_{T}>18 \mathrm{GeV} / c$ with a stub in the CMX matching to its extrapolation within $\Delta_{x C M X}<10 \mathrm{~cm}$.

\section{Trigger Efficiency}

The triggers are very efficient, but they can not take events perfectly. Thus, we have to estimate trigger efficiency for each trigger path and apply this estimation to the Monte Carlo simulation. The trigger efficiency is estimated using the data triggered by a different path, applying the trigger selection cuts, and counting how often events are accepted by our target trigger path.

The followings are the estimated trigger efficiency used for this analysis.

The ELECTRON_CENTRAL_18 trigger efficiency is $96.8 \pm 0.4 \%$, the MUON_CMUP18 trigger efficiency is $88.4 \pm 0.5 \%$, and the MUON_CMX18 trigger efficiency is $91.0 \pm 0.5 \%$.

\subsection{Event Selection}

To select candidate events of the $g g \rightarrow H \rightarrow \ell \nu j j$ process, the following criteria are required:

- Trigger:

Events must be triggered by the specific triggers as discussed in the previous section.

- Primary vertex position:

The $z$-coordinate of the reconstructed primary vertex must be within $60 \mathrm{~cm}$ from the center of the detector.

- Presence of exactly one isolated lepton:

The leptonic decay of a $W$ boson produces exactly one high $p_{T}$ lepton, which must be identified. We require $p_{T}>20 \mathrm{GeV} / c$ for the lepton candidate. If there exist more than one leptons, such events are rejected.

- Difference between the primary vertex and lepton track intercept:

The difference $\left|z_{\text {track }}-z_{0}\right|<5 \mathrm{~cm}$.

- Photon conversion veto:

Discussed in electron identification section. 
- Cosmic ray veto:

Discussed in muon identification section.

- $Z$ boson veto:

If one of the leptons is not identified correctly, $Z \rightarrow \ell^{+} \ell^{-}$events may become background. To remove such events, the invariant mass of the lepton and any track with opposite charge must not be in the $Z$ boson mass window $\left(76-106 \mathrm{GeV} / c^{2}\right)$.

- Large missing transverse energy:

$E_{T}>20 \mathrm{GeV}$. The $E_{T}$ is corrected for the presence of muons (including muon-like isolated tracks) and jet energy correction.

- Jet:

The jets are identified using the cone algorithm with a cone of 0.4 [32] and are required to be central $(|\eta|<2.0)$ with $E_{T}>20 \mathrm{GeV}$. We use exactly 2-jet events.

- QCD veto:

To suppress the non- $W$ QCD background, we apply specific cuts. In order to reduce QCD backgrounds that come from QCD multijet events, we further apply the following cuts:

$$
\begin{aligned}
& M_{T}(e, \nu)>20 \mathrm{GeV} \quad \text { or } \quad M_{T}(\mu, \nu)>10 \mathrm{GeV} \\
& \Delta \phi\left(\mathbb{E}_{T}, j e t 1\right)>1.9-\mathbb{E}_{T} /(20 \mathrm{GeV}) \\
& \Delta \phi\left(\mathbb{E}_{T}, j e t 2\right)>1.8-\mathbb{E}_{T} /(25 \mathrm{GeV})
\end{aligned}
$$

where jet 1 and jet 2 refer to the leading and second leading jet in the event.

- Further QCD veto:

We require $60<M_{T}\left(l, \mathbb{E}_{T}\right)<100 \mathrm{GeV}$, in order to further reduce the non- $W$ background. 



\section{Chapter 5}

\section{Background and Signal Estimation}

Our target signature has one high $p_{T}$ lepton, large missing $E_{T}$ and two high $E_{T}$ jets in the final state. There are background events which decay into the same final state mimicking the signal signature. In this chapter, we present our method to estimate the signal and backgrounds.

\subsection{Background}

The following background sources are considered.

- $W+$ jets Backgrounds: This backgrounds come from $W$ boson production associated with jets. This is the main background source in our analysis.

- Electroweak Backgrounds: Electroweak backgrounds include $t \bar{t}$, single top, $W W, W Z$, $Z Z$ and $Z+$ jets production events. These backgrounds are non-negligible but smaller than the $W+$ jets backgrounds.

- Non- $W$ QCD:A $W$-like signature is generated when one jet fakes a high $p_{T}$ lepton and $\mathbb{E}_{T}$ is generated through jet energy mismeasurement. This background is modeled using the sideband distribution in a QCD enriched data sample. Non- $W$ background is modeled using data sample which false at least two non-kinematic central electron selection (see Table 3.1) which are $E_{\text {had }} / E_{\mathrm{EM}}, \chi_{\text {strip }}^{2}, L_{\mathrm{shr}}, Q \times \Delta_{\text {wire }}$.

\subsection{Likelihood Composition}

In order to separate the signal from background, maximizing the sensitivity of the analysis, we compose a likelihood discriminant.

Suppose we have histograms of a certain variable for signal and background. We call such histograms signal and background templates, and these histograms are normalized so that the total area is 1 . Suppose an (data) event has a value $x_{i}$, and let us denote the content of the bin containing $x_{i}$ in signal histogram $S_{i}$ and that in background histogram $B_{i}$. We define the likelihood for this single variable to be:

$$
L_{i}=\frac{S_{i}}{B_{i}}
$$

By having such likelihood, combining several different separation variables becomes straightforward.

$$
L=\prod_{i} L_{i}=\prod_{i} \frac{S_{i}}{B_{i}}
$$


or

$$
\log L=\sum_{i} \log L_{i}=\sum_{i} \log \left(\frac{S_{i}}{B_{i}}\right)
$$

The suffix $i$ runs through the variables we consider to separate between signal and background. Overflow and underflow are treated as separate bin in our template.

Since the signal behavior changes as the assumed Higgs boson mass changes, we compose several signal templates for the Higgs boson masses in the range we search for. In the current analysis, we search for the Higgs boson with masses 150, 160,170, 180, 190 and $200 \mathrm{GeV} / \mathrm{c}^{2}$. Therefore, we composed six different signal templates.

As for the background, the best possible way to compose the template would be to include all kinds of background processes well modelled by Monte Carlo generators. We instead composed the background templates by $W+$ jets ALPGEN Monte Carlo events alone for simplicity. In facts the contribution from other background processes is small and the distribution is barely independent to them.

We consider the following six variables as the separation variables .

- Reconstructed Higgs mass

- Dijet mass

- $\Delta R($ lepton, Down-type jet)

- $\Delta R($ up-type jet, down-type jet)

- $\mid \Delta \phi\left(\right.$ lepton,$\left.W_{\text {hadronic }}\right) \mid$

- $P_{T}^{\text {lepton }}$

Figure 5.1, 5.2 and 5.3 show the signal and background templates at $m_{H}=180 \mathrm{GeV} / \mathrm{c}^{2}$. In appendix A, we show figures of the signal and backgrounds templates for the other mass points.
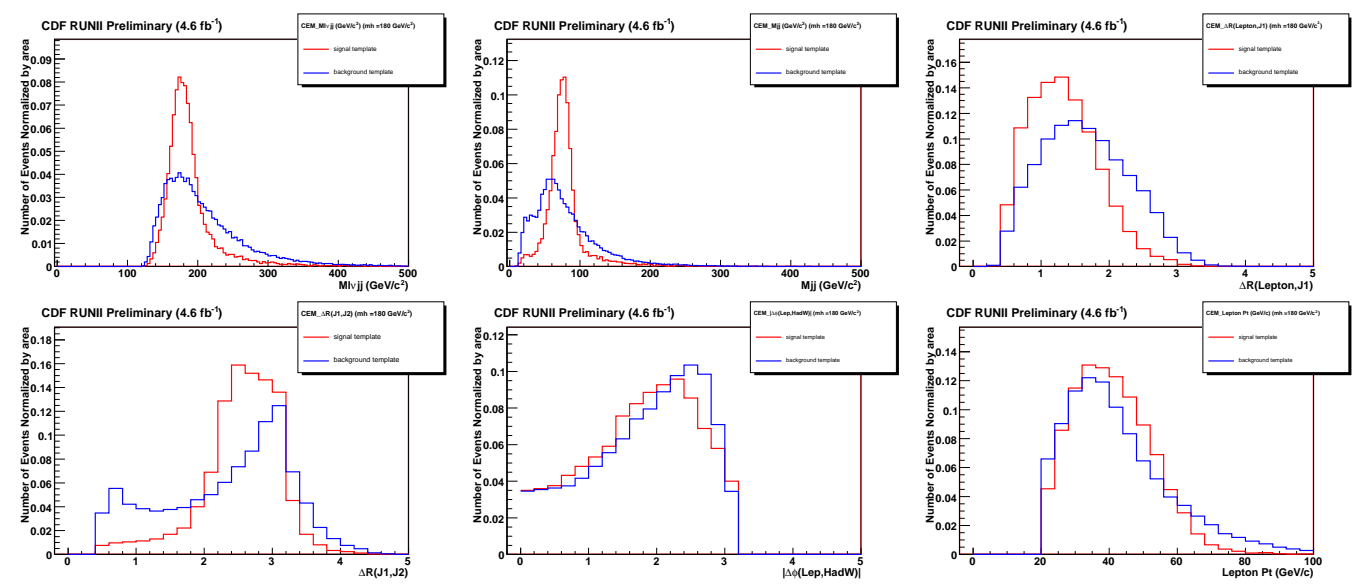

Figure 5.1: Likelihood templates for the CEM. (Red) $H \rightarrow W W, m_{H}=180 \mathrm{GeV} / \mathrm{c}^{2}$, (blue) background. Top column from left: reconstructed higgs mass, dijet mass, $\Delta R$ (lepton, Down-type jet). Bottom from left: $\Delta R$ (up-type jet, down-type jet), $\mid \Delta \phi\left(\right.$ lepton, $\left.W_{\text {hadronic }}\right) \mid, P_{T}^{\text {lepton }}$. 

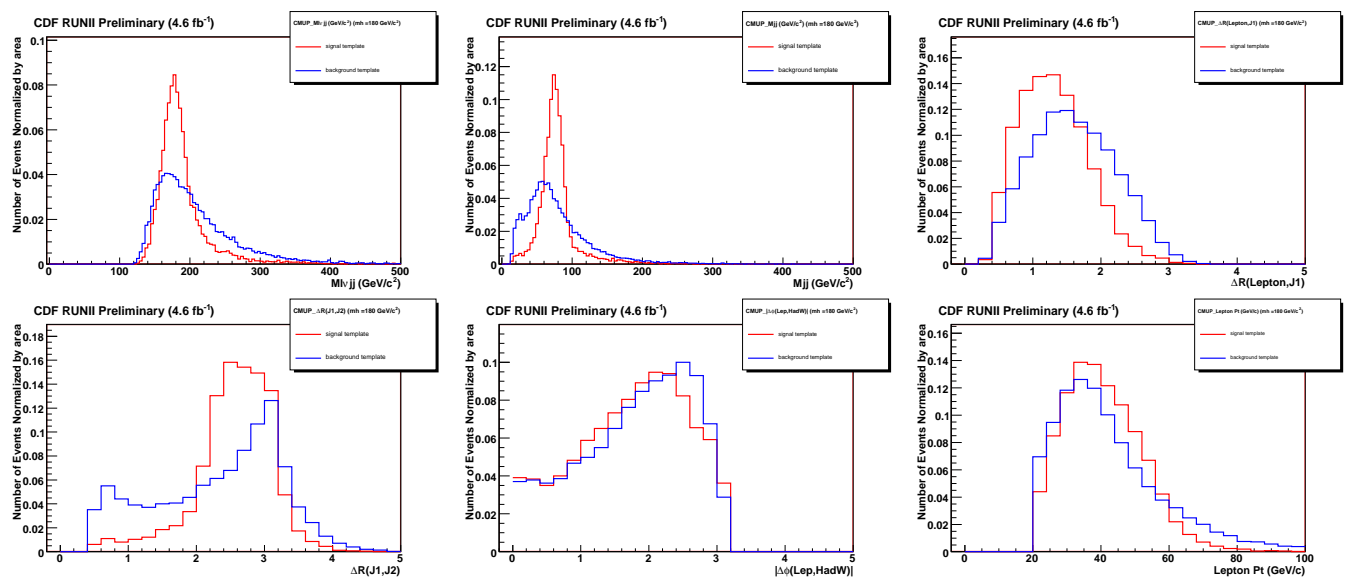

Figure 5.2: Likelihood templates for the CMUP. (Red) $H \rightarrow W W, m_{H}=180 \mathrm{GeV} / \mathrm{c}^{2}$, (blue) background. Top column from left: reconstructed higgs mass, dijet mass, $\Delta R$ (lepton, Down-type jet). $\quad$ Bottom from left: $\Delta R$ (up-type jet, down-type jet), $\mid \Delta \phi\left(\right.$ lepton,$\left.W_{\text {hadronic }}\right) \mid, P_{T}^{\text {lepton }}$.
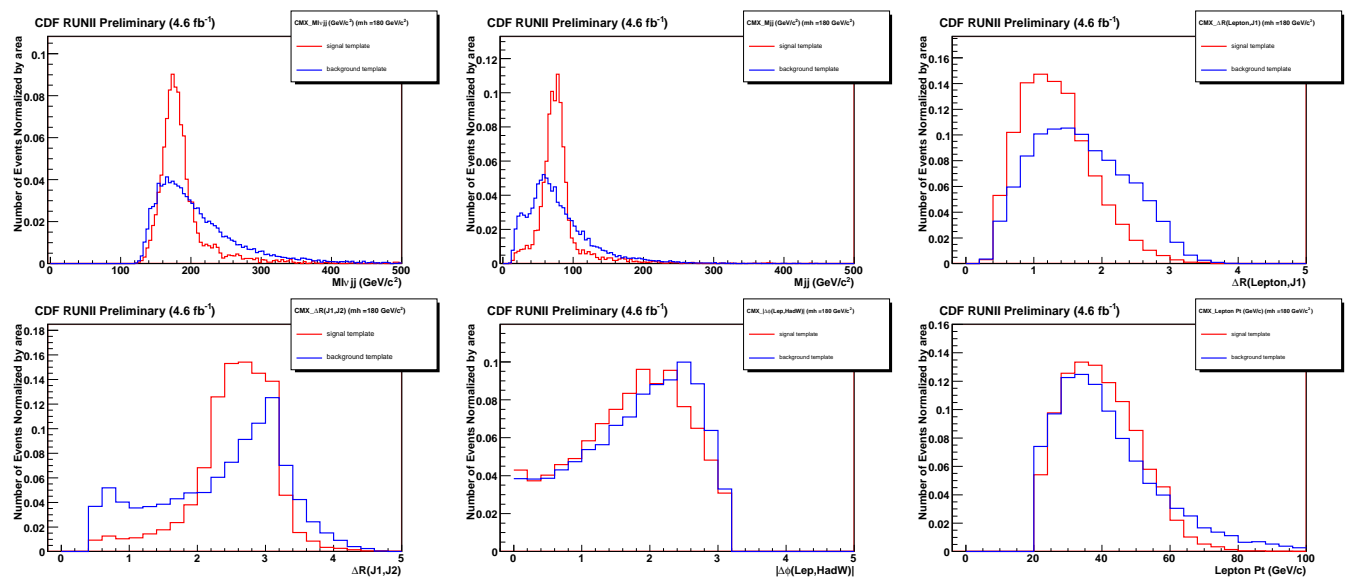

Figure 5.3: Likelihood templates for the CMX. (Red) $H \rightarrow W W, m_{H}=180 \mathrm{GeV} / \mathrm{c}^{2}$, (blue) background. Top column from left: reconstructed higgs mass, dijet mass, $\Delta R$ (lepton, Down-type jet). $\quad$ Bottom from left: $\Delta R$ (up-type jet, down-type jet), $\mid \Delta \phi\left(\right.$ lepton,$\left.W_{\text {hadronic }}\right) \mid, P_{T}^{\text {lepton }}$. 


\subsection{Method and the Result of Background and Signal Estimation}

In our analysis, we first fit the missing $E_{T}$ distribution using the shapes of the electroweak, non$W, W+$ jets, $Z+$ jets backgrounds, with the signal fluctuated. The missing $E_{T}$ distribution before applying the $\mathbb{E}_{T}>20 \mathrm{GeV}$ and $60<M_{T}<100 \mathrm{GeV}$ cuts is used as the control region for the non- $W$ background. The number of the electroweak backgrounds ( $t \bar{t}$, single top, $W W / W Z / Z Z$ diboson processes and $Z+$ jets through electroweak processes) are normalized to the cross sections listed in Table5.1 and are fixed in the in the fit. The initial normalization of signal is set to the cross section predicted by SM (Table 5.2). Under the above condition, we extract the normalization for the overall $W+$ jets background as well as the normalization for non- $W$ background.

Table 5.1: Cross sections of the electroweak background processes.

\begin{tabular}{cc}
\hline \hline process & cross section $(\mathrm{pb})$ \\
\hline$W W$ & $11.34_{-0.49}^{+0.56}(\text { scale })_{-0.28}^{+0.35}(P D F)$ \\
$W Z$ & $3.22_{-0.17}^{+0.20}(\text { scale })_{-0.08}^{+0.11}(P D F)$ \\
$Z Z$ & $1.20_{-0.04}^{+0.05}(\text { scale })_{-0.03}^{+0.04}(P D F)$ \\
single top (s-chan.) & $1.046 \pm 0.006($ scale $) \pm 0.059(P D F) \pm 0.030$ (mass $)$ \\
single top (t-chan.) & $2.10 \pm 0.027($ scale $) \pm 0.018(P D F) \pm 0.045($ mass $)$ \\
$t \bar{t}$ & $7.04_{-0.36}^{+0.24}($ scale $) \pm 0.014(P D F) \pm 0.030($ mass $)$ \\
\hline$Z+0$ parton & 158 \\
$Z+1$ parton & 21.6 \\
$Z+2$ parton & 3.47 \\
$Z+3$ parton & 0.548 \\
$Z \geq 4$ parton & 0.0992 \\
\hline \hline
\end{tabular}

Table 5.2: Cross sections for the signal events, generated with PYTHIA parameters PARP $(91)=$ 2.10 and $\operatorname{PARP}(64)=0.2$.

\begin{tabular}{ccc}
\hline \hline process $g g \rightarrow H \rightarrow W W$ & $g g \rightarrow H$ cross section $(\mathrm{pb})$ & $\mathrm{BR}\left(H \rightarrow W^{+} W^{-}\right)(\%)$ \\
\hline$m_{H}=150 \mathrm{GeV} / \mathrm{c}^{2}$ & 0.5391 & 68.91 \\
$m_{H}=160 \mathrm{GeV} / \mathrm{c}^{2}$ & 0.4323 & 90.48 \\
$m_{H}=170 \mathrm{GeV} / \mathrm{c}^{2}$ & 0.3440 & 96.39 \\
$m_{H}=180 \mathrm{GeV} / \mathrm{c}^{2}$ & 0.2792 & 93.25 \\
$m_{H}=190 \mathrm{GeV} / \mathrm{c}^{2}$ & 0.2280 & 78.70 \\
$m_{H}=200 \mathrm{GeV} / \mathrm{c}^{2}$ & 0.1891 & 74.26 \\
\hline \hline
\end{tabular}

In the second step, we fit our final discriminant log likelihood distribution in the signal region. In order to estimate the $W+$ jets background modeling, we separate $W+$ jets background and fit for its components independently. These components are defined by the flavors of the final state partons from the hard scattering process (e.g. $W+q q, W+q g / g q, W+g g$ and $W+0$ or 1 parton events coming into the 2 jet bin). The non- $W$ QCD background is modeled using an event sample selected from analysis dataset by requiring that at least two of the non-kinematical electron identification criteria fail. We model other background and signal events by Monte Carlo events. 
We fluctuate the fraction of electroweak, non- $W, 4 W+$ jets subsamples, and the signal in this fit. The fluctuation of these fractions is constrained by their relative uncertainties which are showed in Table 5.3.

We perform the fit to missing $E_{T}$ and log likelihood distribution iteratively until the scaling factor to each background obtained in the fit stabilizes within $3 \%$ of the previous iteration. The fit to missing $E_{T}$ is done with CEM, CMUP and CMX lepton events separately, while the fit to the log likelihood is done after combining the three lepton categories. The smallest fractional uncertainty we quote for the normalization of background events is $\sim 10 \%$ for the electroweak backgrounds. Figure 5.4 and 5.5 show the results of the fits from our final iteration for $m_{H}=180 \mathrm{GeV} / c^{2}$. Table 5.4 summarizes the estimated background and signal event rate by the iteration method at $m_{H}=180 \mathrm{GeV} / c^{2}$. Table 5.5 summerizes the expected number of signal events with the Standerd Model Higgs boson production cross section times branching ratio.

The normalization for the background events are obtained by this process, however, which is not include whole systematic uncertainties. Then we choose the normalization at $m_{H}=180 \mathrm{GeV}$ which is the middle point in our mass range and fit our likelihood distribution with all systematic uncertainties on each mass points. The systematic uncertainties are summarized in chapter 6.

Finally, we obtain the final fit event yield of background which is listed in table 5.6. Figure 5.6 shows distributions of the individual variables in the likelihood with final fit event yield at $m_{H}=180 \mathrm{GeV} / c^{2}$. The background estimation is plotted along with data. Distribution of signal assuming $m_{H}=180 \mathrm{GeV} / \mathrm{c}^{2}$ Standard Model cross section is also shown, which is scaled up by a factor of 100. Figure 5.7 shows the overall likelihood distributions with final fit event yield at $m_{H}=180 \mathrm{GeV} / c^{2}$. In appendix $\mathrm{B}$, we show figures of the individual variables in the likelihood and the overall likelihood distributions for the other mass points.

Table 5.3: Constraints for each background and signal fluctuation in the iteration process.

CDF RUNII Preliminary $\left(4.6 \mathrm{fb}^{-1}\right)$

\begin{tabular}{|c|c|c|c|c|c|c|c|c|}
\hline Systematic Uncertainty (\%) & signal & Electroweak & $\mathrm{W}+\mathrm{qq}$ & $\mathrm{W}+\mathrm{qg} / \mathrm{gq}$ & $\mathrm{W}+\mathrm{gg}$ & $\mathrm{W}+0 / 1 \mathrm{p}$ & $\mathrm{Z}+\mathrm{LF}$ & non-W \\
\hline Luminosity & & $\overline{76}$ & & & & & & \\
\hline Trig. Eff., Lepton ID & & 2 & & & & & & \\
\hline Ewk Cross Section & & 8 & & & & & & \\
\hline Signal cross section & 1000 & & & & & & & \\
\hline ISR/FSR, PDF & & & & & & & & \\
\hline Jet Energy Scale & & 2 & & & & & & \\
\hline W+qq Normalization & & & 20 & & & & & \\
\hline W+qg/gq Normalization & & & & 20 & & & & \\
\hline W+gg Normalization & & & & & 20 & & & \\
\hline $\mathrm{W}+0 / 1 \mathrm{p}$ Normalization & & & & & & 20 & & \\
\hline Z+jets Normalization & & & & & & & 40 & \\
\hline non-W Normalization & & & & & & & & 40 \\
\hline
\end{tabular}



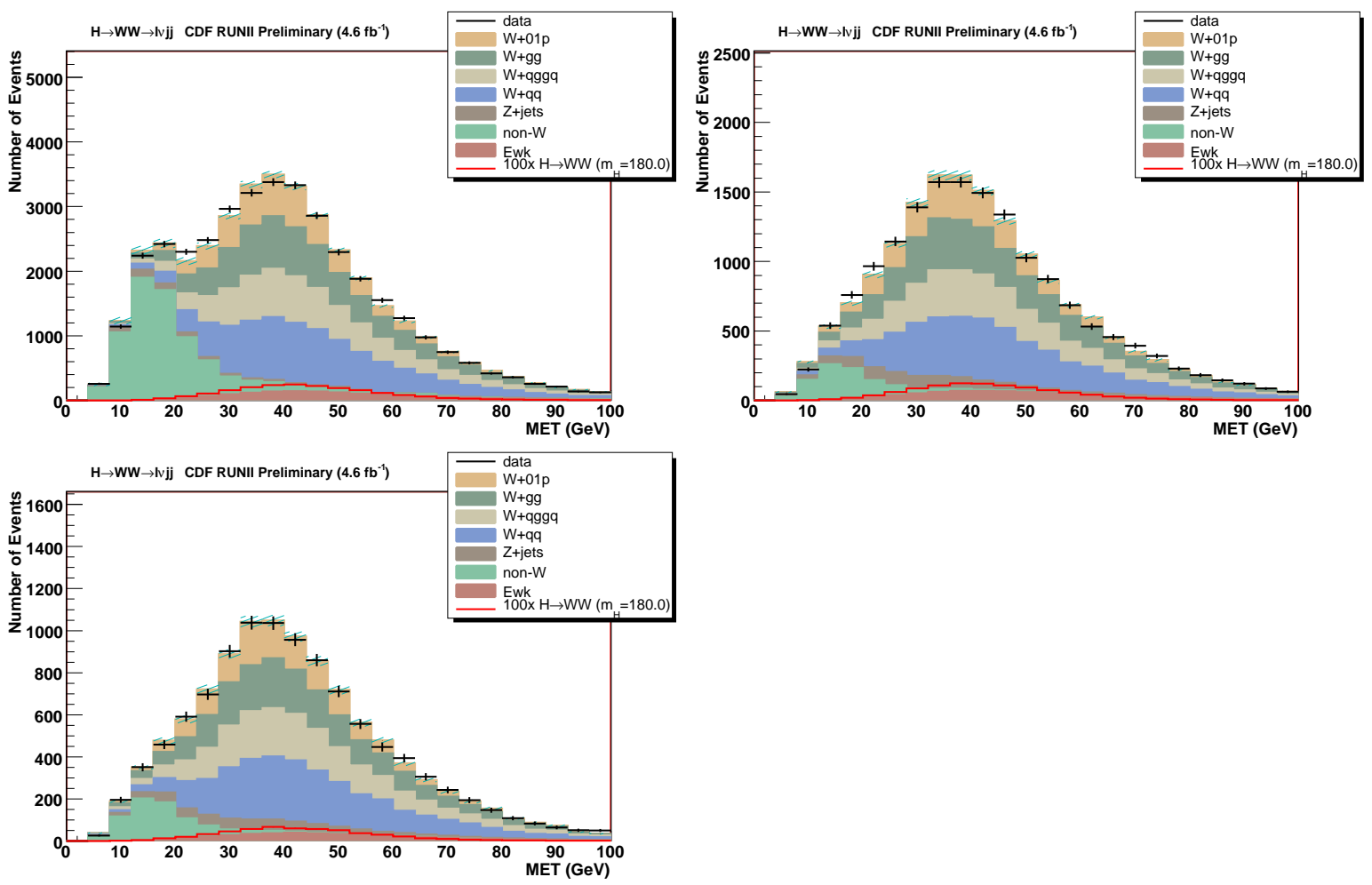

Figure 5.4: Fit to $\mathbb{E}_{T}$ distribution to extract the normalization for the non- $W$ and overall $W+$ jets backgrounds. Events with CEM electrons, CMUP and CMX muons are plotted in top left, top right, and bottom left separately. Red line shows the signal distribution with SM cross section times a hundred.

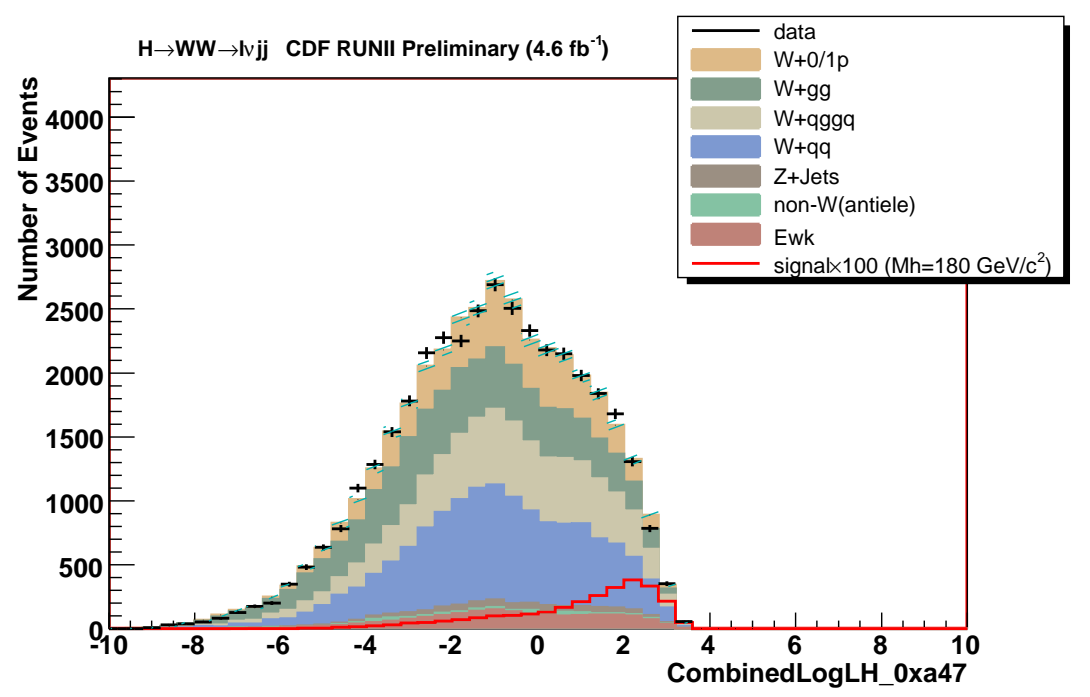

Figure 5.5: Fit to log likelihood distribution in the final iteration, for an assumed Higgs boson mass of $180 \mathrm{GeV} / \mathrm{c}^{2}$. Red line shows the signal distribution with SM cross section times a hundred. 
Table 5.4: Estimates of the background events by the iteration for each mass point. The electroweak subprocesses are $W W, W Z, Z Z$, single top (t-chan. and s-chan.) and $t \bar{t}$.

\begin{tabular}{cc}
\multicolumn{2}{c}{ CDF RUNII Preliminary $\left(4.6 \mathrm{fb}^{-1}\right)$} \\
\hline \hline Higgs Mass $\left(\mathrm{GeV} / \mathrm{c}^{2}\right)$ & 180 \\
\hline process & num. of events \\
\hline Ewk & 1910 \\
non-W QCD & 368 \\
Z+jets & 814 \\
W+qq & 11538 \\
W+qggq & 8259 \\
W+gg & 8549 \\
W+0/1p & 6270 \\
\hline Total Background & 37708 \\
\hline Observed Events & 37670 \\
\hline signal & $\leq 41(68 \%$ C.L. $)$ \\
\hline \hline
\end{tabular}


Table 5.5: Expected number of the signal events with the Standard Model cross section for each mass point.

CDF RUNII Preliminary $\left(4.6 \mathrm{fb}^{-1}\right)$

\begin{tabular}{ccccccc}
\hline \hline Higgs Mass $\left(\mathrm{GeV} / \mathrm{c}^{2}\right)$ & 150 & 160 & 170 & 180 & 190 & 200 \\
\hline Expected number of events & $22.0 \pm 6.5$ & $38.6 \pm 11.1$ & $36.1 \pm 10.3$ & $28.0 \pm 8.0$ & $18.9 \pm 5.4$ & $14.9 \pm 4.3$ \\
\hline \hline
\end{tabular}


Table 5.6: Final number of Estimated background events. The electroweak subprocesses are $W W, W Z, Z Z$, single top (t-chan. and s-chan.) and $t \bar{t}$.

CDF RUNII Preliminary $\left(4.6 \mathrm{fb}^{-1}\right)$

\begin{tabular}{ccccccc}
\hline \hline Higgs Mass $\left(\mathrm{GeV} / \mathrm{c}^{2}\right)$ & 150 & 160 & 170 & 180 & 190 & 200 \\
\hline process & \multicolumn{5}{c}{ num. of events } \\
\hline Ewk & $1929 \pm 170$ & $1927 \pm 169$ & $1909 \pm 168$ & $1884 \pm 166$ & $1916 \pm 168$ & $1903 \pm 166$ \\
non-W QCD & $426 \pm 160$ & $342 \pm 126$ & $327 \pm 121$ & $325 \pm 122$ & $292 \pm 109$ & $399 \pm 150$ \\
Z+jets & $834 \pm 327$ & $854 \pm 335$ & $880 \pm 344$ & $880 \pm 345$ & $833 \pm 327$ & $759 \pm 300$ \\
W+qq & $12680 \pm 1212$ & $12913 \pm 1298$ & $11683 \pm 1131$ & $12912 \pm 1453$ & $11064 \pm 1525$ & $11923 \pm 1460$ \\
W+qggq & $8316 \pm 1346$ & $8927 \pm 1409$ & $7216 \pm 1055$ & $7712 \pm 1045$ & $9314 \pm 1314$ & $8129 \pm 1249$ \\
W+gg & $8122 \pm 412$ & $7753 \pm 389$ & $8000 \pm 403$ & $7788 \pm 452$ & $8436 \pm 612$ & $8688 \pm 1203$ \\
W+0/1p & $5379 \pm 634$ & $4814 \pm 593$ & $7630 \pm 986$ & $6180 \pm 918$ & $5836 \pm 875$ & $5893 \pm 868$ \\
\hline Total Background & $37689 \pm 2004$ & $37534 \pm 2082$ & $37648 \pm 1921$ & $37685 \pm 2101$ & $37693 \pm 2292$ & $37697 \pm 2456$ \\
\hline Observed Events & 37670 & 37670 & 37670 & 37670 & 37670 & 37670 \\
\hline \hline
\end{tabular}



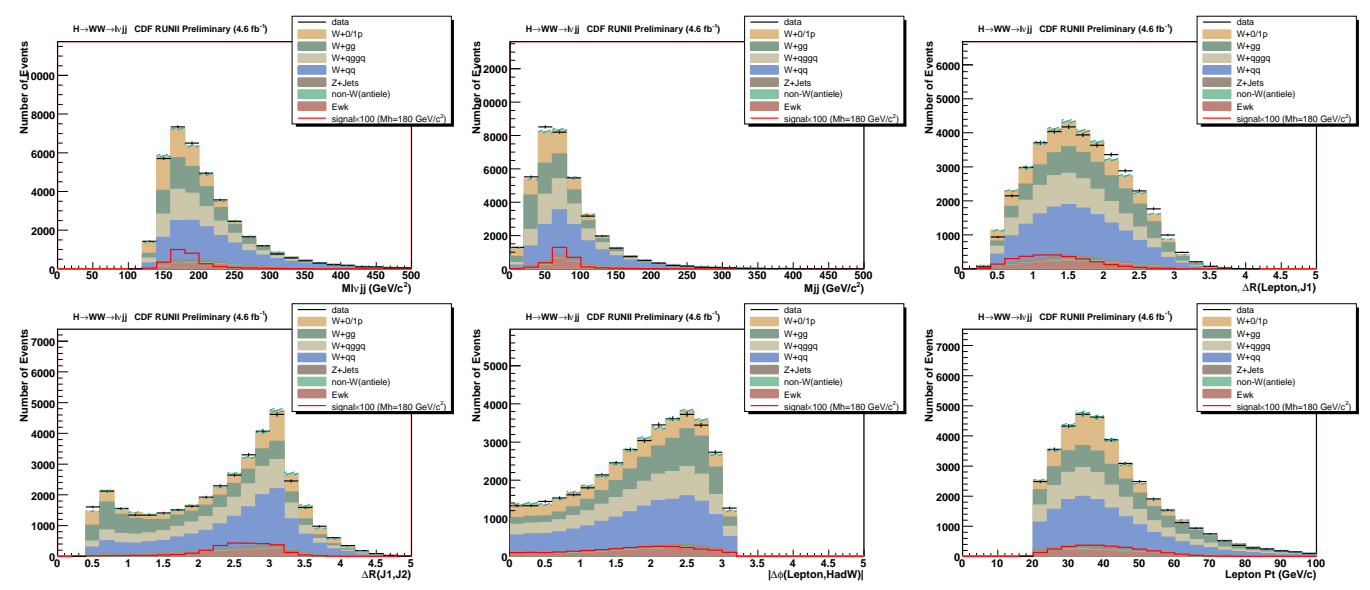

Figure 5.6: Likelihood input distributions with final fit event yield for $m_{H}=180 \mathrm{GeV} / \mathrm{c}^{2}$. The crosses show the data, and filled histograms are for backgrounds. The open red histogram shows the distribution for signal with $m_{H}=180 \mathrm{GeV} / \mathrm{c}^{2}$, scaled by a factor of 100 . Top column from left: reconstructed higgs mass, dijet mass, $\Delta R$ (lepton, Down-type jet). Bottom from left: $\Delta R$ (up-type jet, down-type jet), $\mid \Delta \phi$ (lepton, $\left.W_{\text {hadronic }}\right) \mid, P_{T}^{\text {lepton }}$. 


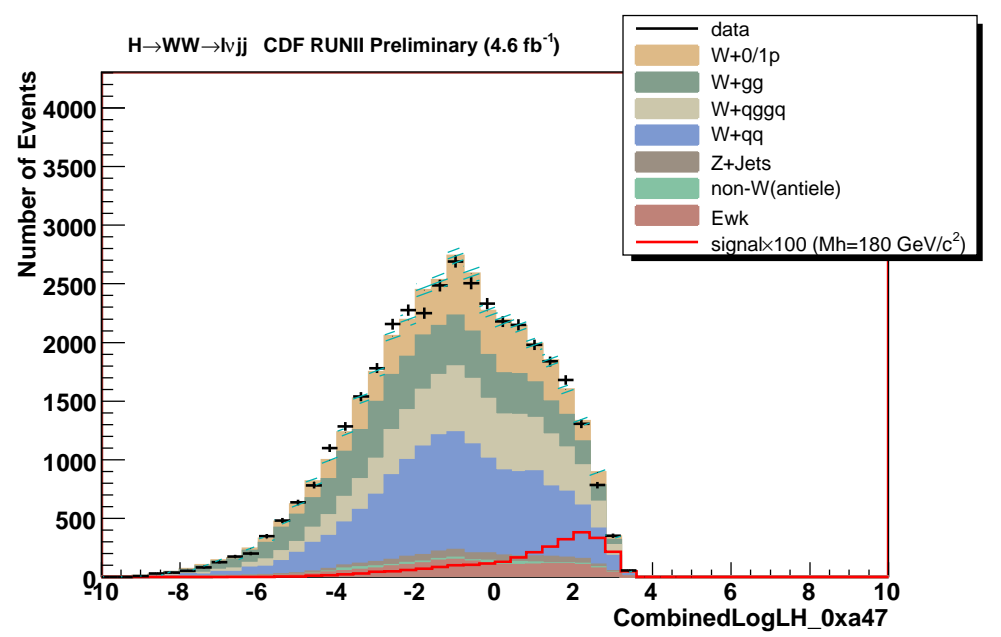

Figure 5.7: Likelihood output distribution with final fit event yield for $m_{H}=180 \mathrm{GeV} / \mathrm{c}^{2}$. The black dot with errors shows the data, filled histograms are for backgrounds. The open red histogram shows the distribution for signal with $m_{H}=180 \mathrm{GeV} / \mathrm{c}^{2}$ assumed, scaled by 100 . 



\section{Chapter 6}

\section{Systematic Uncertainties}

The sources of the systematic uncertainty are considered that affect the rates of the signal and background, as well the shapes of the signal and background distributions. In this study, we consider the uncertainties for the jet energy scale and $Q^{2}$ of the $W+$ jets ALPGEN Monte Carlo, which could have sizeable effects on the distribution shapes. We first summarize the systematic uncertainties that affect the event rate, followed by description of the systematic effect on the distribution shapes.

\subsection{Rate Uncertainties}

We estimated the signal uncertainties for an assumed Higgs boson mass of $170 \mathrm{GeV} / \mathrm{c}^{2}$. The obtained uncertainties are applied to analyses at different Higgs masses.

\subsubsection{Luminosity}

Signal, electroweak and $\mathrm{Z}+$ jets backgrounds are normalized to the theoretical cross sections times the integrated luminosity of the dataset, which are subject to the luminosity uncertainty. We assigned 4.4\%(CDF) and 3.8(Tevatron) [35] of uncertainties on the event rates of these processes.

Normalization of non- $W$ and $W$ +jets are determined by comparison to data, and these processes are not subject to the luminosity uncertainty.

\subsubsection{Trigger Efficiency and Lepton Reconstruction Efficiency}

We applied scale factors for the trigger and lepton reconstruction efficiencies in order to match the efficiencies in signal Monte Carlo studies to those in data. We calculated the average of the scale factors weighted by luminosity over the data periods we analyze (up to period 24) and applied that number to the Monte Carlo efficiency. The uncertainties on the scale factors were calculated at the same time, and we found them to be $0.3 \%$ for trigger, and $0.3 \%$ for the lepton reconstruction. Almost all events of Electroweak background have a high $P_{\mathrm{T}}$ lepton. Then, the uncertainties for Electroweak background are considered same level for signal. We assign $2 \%$ uncertainty conservatively to signal and Electroweak background.

\subsubsection{Signal Cross Section}

$g g \rightarrow H$ channel is a QCD process the corresponding theoretical uncertainty is not small. We use recent studies with the HNNLO program [36-38] to assign theoretical uncertainties based on 
observed changes in the cross section originating from modications to the renormalization and factorization scales used in the calculation and the input PDF model. In the case of PDF model uncertainties, we use MSTW 2008 NNLO PDF set [39] eigenfunctions, which also account for potential variations originating from uncertainty in the value of $\alpha_{s}\left(q^{2}\right)$.

\subsubsection{Jet Energy Scale Uncertainty}

We changed the correction factor by $\pm 1 \sigma$ around the center value, and assigned the observed shift in acceptance as the systematic uncertainty. We measured the uncertainty on the acceptance due to the uncertainty on the jet energy scale to signal, electroweak and $\mathrm{W}+$ jets background processes. The results are summarized in Table6.1.

\subsection{5 non- $W$ normalization}

The uncertainty on the non- $W$ scaling is $40 \%$ [30].

\subsection{6 $W+$ jets normalization}

As stated earlier, we obtain the $W+$ jets normalization by a MET fit outside our $M_{T}$ window. We also estimated the normalization without applying any requirement on $M_{T}$. Such an alternative estimation shifted the normalization by $+3 \%$. The statistical uncertainty of the $W+$ jets normalization which come from MET fit is $5 \%$. We assign a $6 \%$ uncertainty on the normalization of $W+$ jets background. Finally, we separate $W+$ jets sample to four categories which are quarkquark, quark-gluon and gluon-gluon pairs and events with zero or one parton using information of hard processes in HEPG. Each $W+$ jets group has $1 / 5 \sim 1 / 3$ statistics of all amount of $W+$ jets. Then $20 \%$ is enough to cover the uncertainty for each $W+$ jets category.

\subsection{7 $Z+$ jets normalization}

$40 \%$ were applied to $Z+$ jets normalization. It is enough to cover the uncertainty for $Z+$ jets [40].

\subsubsection{ISR/FSR}

We analyzed a signal Monte Carlo that has a different parton shower settings than the nominal one. We measured the change in the acceptance, and assigned $2.7 \%$.

\section{Summary of rate systematics}

Table 6.1 summarize all the systematic uncertainties that affect the estimated event rate. 
Table 6.1: Relative systematic uncertainties on the event rates. Subscript s means the shape related systematics.

CDF RUNII Preliminary $\left(4.6 \mathrm{fb}^{-1}\right)$

\begin{tabular}{|c|c|c|c|c|c|c|c|c|}
\hline Systematic Uncertainty (\%) & signal & Electroweak & $\mathrm{W}+\mathrm{qq}$ & $W+q g / g q$ & $\mathrm{~W}+\mathrm{gg}$ & $\mathrm{W}+0 / 1 \mathrm{p}$ & $\mathrm{Z}+\mathrm{LF}$ & non-W \\
\hline Luminosity (CDF) & 4.4 & 4.4 & & & & & 4.4 & \\
\hline Luminosity (Tevatron) & 3.8 & 3.8 & & & & & 3.8 & \\
\hline Trig. Eff., Lepton ID & 2 & 2 & & & & & 2 & \\
\hline Ewk Cross Section & & 6 & & & & & & \\
\hline \multicolumn{9}{|l|}{ Signal cross section } \\
\hline Scale inclusive & 13.4 & & & & & & & \\
\hline Scale1+Jet & -23 & & & & & & & \\
\hline Scale2+Jet & 0 & & & & & & & \\
\hline PDF & 7.6 & & & & & & & \\
\hline ISR/FSR & 2.7 & & & & & & & \\
\hline Jet Energy Scale & $3 \sim 8$ & 2 & & & & & & \\
\hline W+qq Normalization & & & 20 & & & & & \\
\hline $\mathrm{W}+\mathrm{qg} / \mathrm{gq}$ Normalization & & & & 20 & & & & \\
\hline W+gg Normalization & & & & & 20 & & & \\
\hline $\mathrm{W}+0 / 1$ parton Normalization & & & & & & 20 & & \\
\hline $\mathrm{W}+\mathrm{jets}$ JES quark $+1 \sigma$ & & & $+0.6 \mathrm{~s}$ & $-2.8 \mathrm{~s}$ & $-5.5 \mathrm{~s}$ & $+19.4 \mathrm{~s}$ & & \\
\hline $\mathrm{W}+\mathrm{jets}$ JES quark $-1 \sigma$ & & & $-2.0 \mathrm{~s}$ & $+2.1 \mathrm{~s}$ & $+5.8 \mathrm{~s}$ & $-12.6 \mathrm{~s}$ & & \\
\hline $\mathrm{W}+$ jets JES gluon $+1 \sigma$ & & & $-8.5 \mathrm{~s}$ & $-0.5 \mathrm{~s}$ & $+7.0 \mathrm{~s}$ & $+15.3 \mathrm{~s}$ & & \\
\hline $\mathrm{W}+$ jets JES gluon $-1 \sigma$ & & & $+9.1 \mathrm{~s}$ & $-0.7 \mathrm{~s}$ & $-9.0 \mathrm{~s}$ & $-9.0 \mathrm{~s}$ & & \\
\hline $\mathrm{W}+$ jets $\mathrm{Q}^{2}$ shape $Q^{2}=2.0$ & & & $+0.2 \mathrm{~s}$ & $-6.1 \mathrm{~s}$ & $+0.8 \mathrm{~s}$ & $+22.9 \mathrm{~s}$ & & \\
\hline $\mathrm{W}+$ jets $\mathrm{Q}^{2}$ shape $Q^{2}=0.5$ & & & $+0.7 \mathrm{~s}$ & $+9.7 \mathrm{~s}$ & $-4.1 \mathrm{~s}$ & $-34.3 \mathrm{~s}$ & & \\
\hline Z+jets Normalization & & & & & & & 39.5 & \\
\hline non-W Normalization & & & & & & & & 40 \\
\hline
\end{tabular}




\subsection{Shape systematics}

\subsubsection{Jet energy scale in signal}

We estimate shape systematical uncertainties related to JES for events of signal and find that JES systematical uncertainties have little effect for our log likelihood distribution. Therefor we don't apply shape systematical uncertainties related to JES.

\subsubsection{Jet energy scale in $W+$ jets background}

We also estimate shape systematical uncertainty related to JES for $W+$ jets background. We have 4 kind fo JES systematics which are $(q+1 \sigma, g \pm 0 \sigma),(q-1 \sigma, g \pm 0 \sigma),(q \pm 0 \sigma, g+1 \sigma)$ and $(q \pm 0 \sigma, g-1 \sigma)$. Figure 6.1-6.4 show distributions of the likelihood output for $W+$ jets samples. We show figures of JES shape systematics for the other Higgs mass points in appendix C.
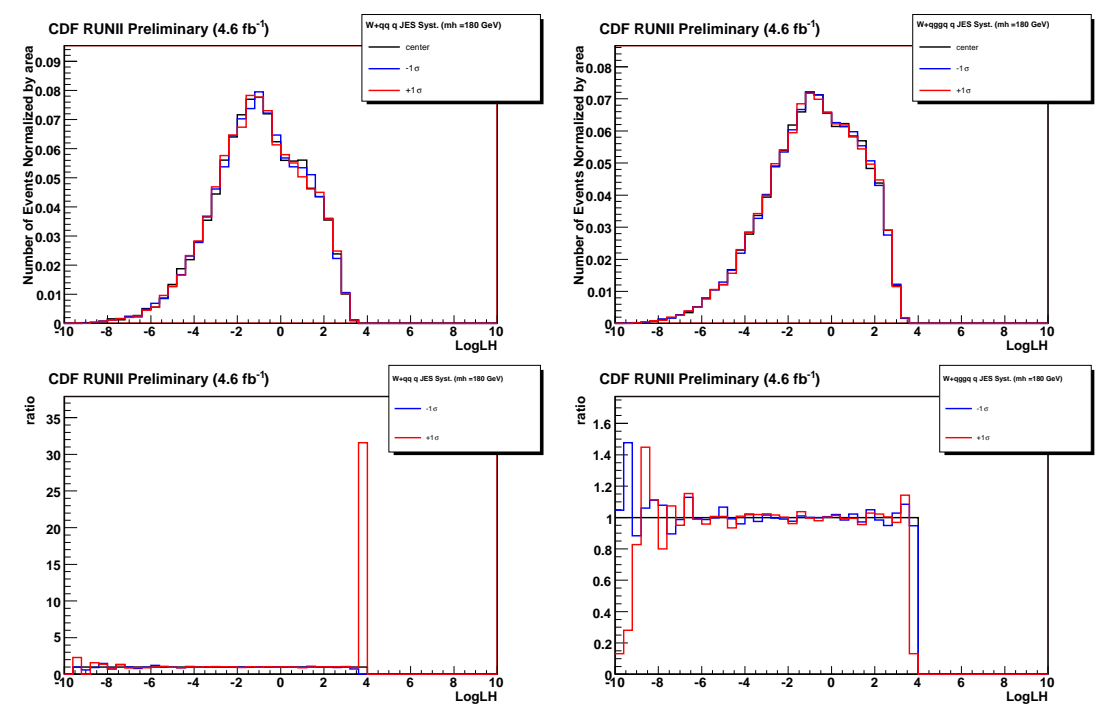

Figure 6.1: Likelihood output distribution using the $W+$ jets quark JES $+1 \sigma$ systematic samples at $m_{H}=180 \mathrm{GeV} / \mathrm{c}^{2}$. The black line shows the center value. These histograms show the distribution for $+1 \sigma$ (red) and $-1 \sigma$ (blue). The left top plot shows log likelihood output for $W+\mathrm{qq}$ background. The left bottom plot shows ratios which are $\pm 1 \sigma /($ center value). The right plots show log likelihood output for $W+$ qggq background.

\subsection{3 $Q^{2}$ of $W$ +jets background}

The center value of $\mathrm{Q}^{2}=m_{W}^{2}$ for the $W+$ jets Monte Carlo samples. We estimate shape systematical uncertainties of the $W+$ jets using Monte Carlo samples with $2.0 \times \mathrm{Q}^{2}$ and $0.5 \times \mathrm{Q}^{2}$. Figure 6.5 and 6.6 show distributions of the likelihood output for $W+$ jets samples. We show figures of $\mathrm{Q}^{2}$ shape systematics of the other Higgs mass points in appendix $\mathrm{C}$. 

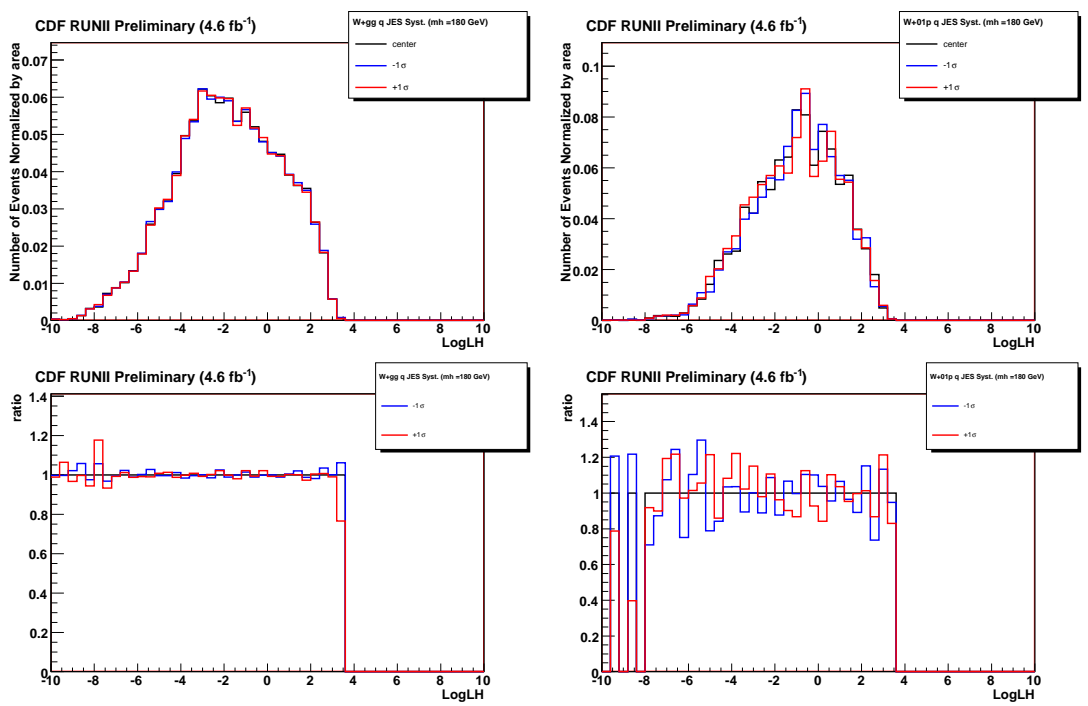

Figure 6.2: Likelihood output distribution using the $W+$ jets quark JES $\pm 1 \sigma$ systematic samples at $m_{H}=180 \mathrm{GeV} / \mathrm{c}^{2}$. The black line shows the center value. These histograms show the distribution for $+1 \sigma$ (red) and $-1 \sigma$ (blue). The left top plot shows log likelihood output for $W+g g$ background. The left bottom plot shows ratios which are $\pm 1 \sigma /($ center value). The right plots show log likelihood output for $W+0$ or 1 parton background.
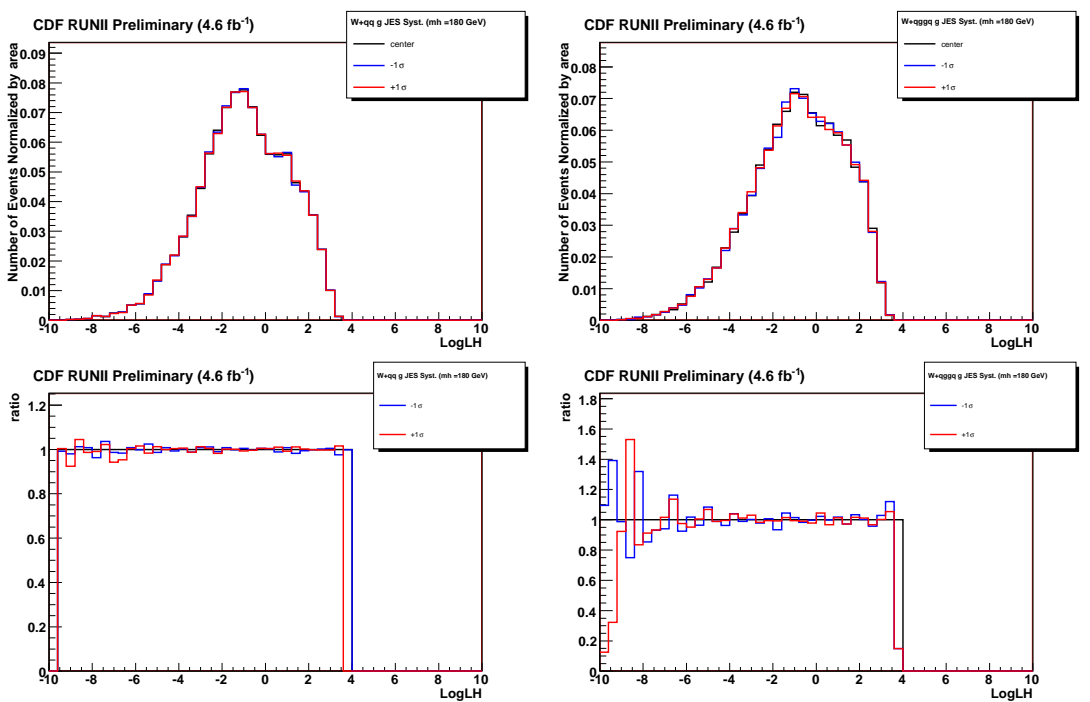

Figure 6.3: Likelihood output distribution using the $W+$ jets gluon JES $+1 \sigma$ systematic samples at $m_{H}=180 \mathrm{GeV} / \mathrm{c}^{2}$. The black line shows the center value. These histograms show the distribution for $+1 \sigma$ (red) and $-1 \sigma$ (blue). The left top plot shows log likelihood output for $W+\mathrm{qq}$ background. The left bottom plot shows ratios which are $\pm 1 \sigma /($ center value). The right plots show log likelihood output for $W+$ qggq background. 

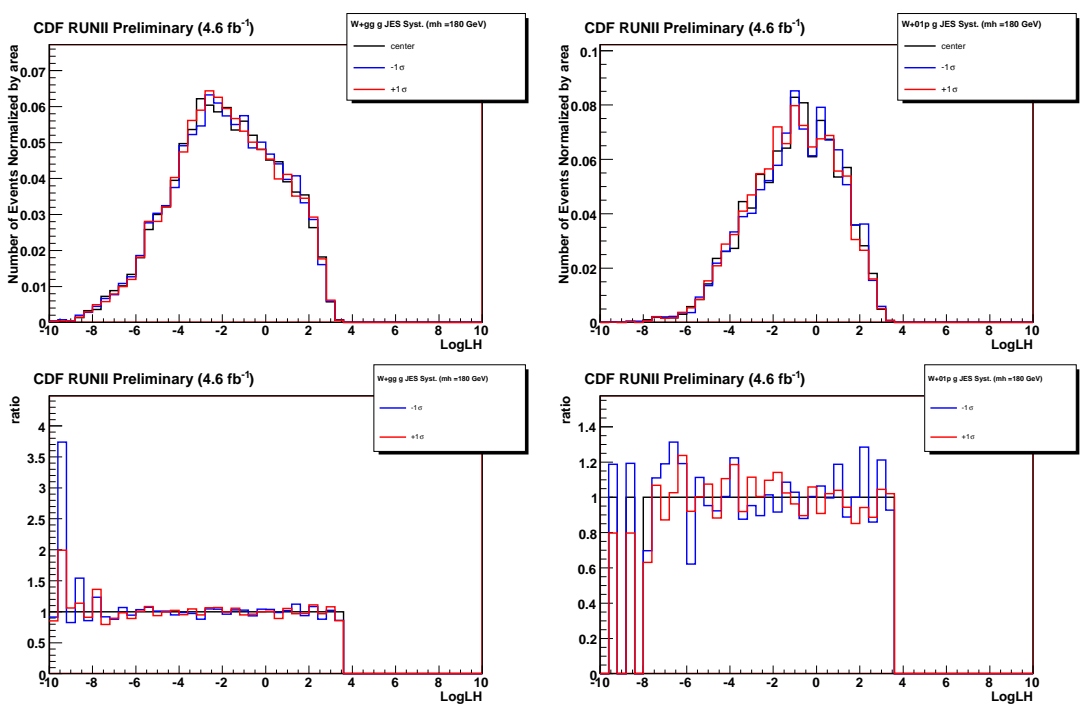

Figure 6.4: Likelihood output distribution using the $W+$ jets gluon JES $\pm 1 \sigma$ systematic samples at $m_{H}=180 \mathrm{GeV} / \mathrm{c}^{2}$. The black line shows the center value. These histograms show the distribution for $+1 \sigma$ (red) and $-1 \sigma$ (blue). The left top plot shows log likelihood output for $W+g g$ background. The left bottom plot shows ratios which are $\pm 1 \sigma$ (center value). The right plot shows $\log$ likelihood output for $W+0$ or 1 parton background.
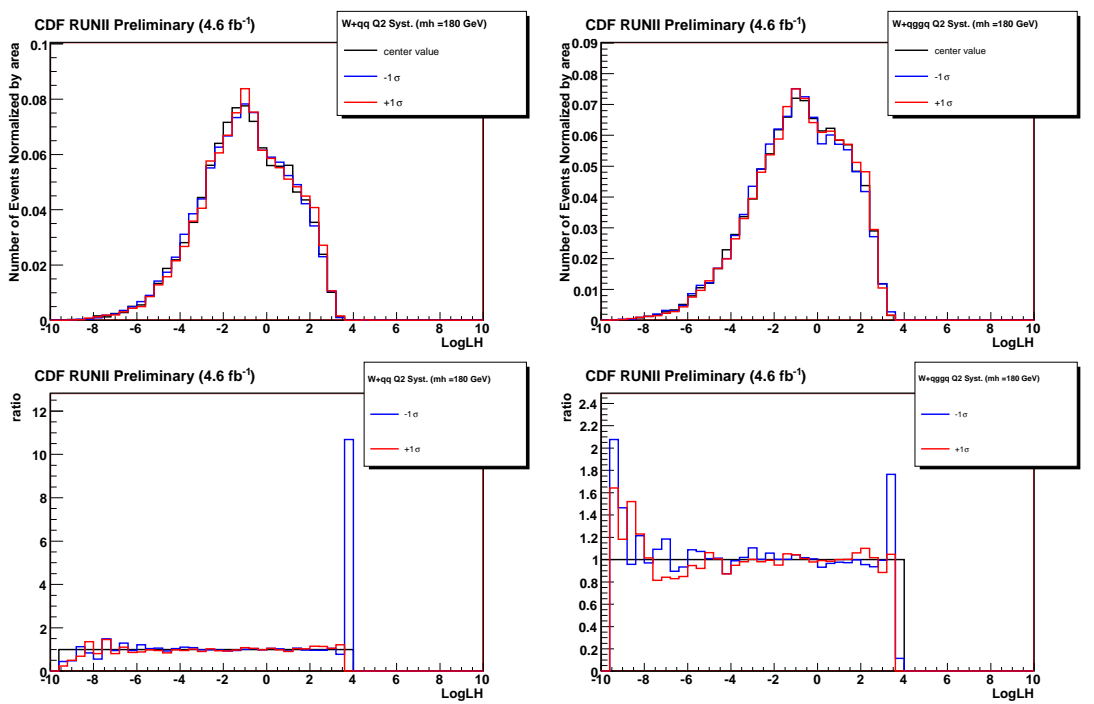

Figure 6.5: Likelihood output distribution using the $W+$ jets $\mathrm{Q}^{2}$ systematic samples at $m_{H}=$ $180 \mathrm{GeV} / \mathrm{c}^{2}$. The black line shows the center value. The red histogram shows the distribution for $+1 \sigma\left(2.0 \times \mathrm{Q}^{2}\right)$. The blue histogram shows the distribution for $-1 \sigma\left(0.5 \times \mathrm{Q}^{2}\right)$. The left top plot shows log likelihood output for $W+\mathrm{qq}$ background. The left bottom plot shows ratios which are $\pm 1 \sigma /$ (center value). The right plots show log likelihood output for $W+q g g q$ background. 

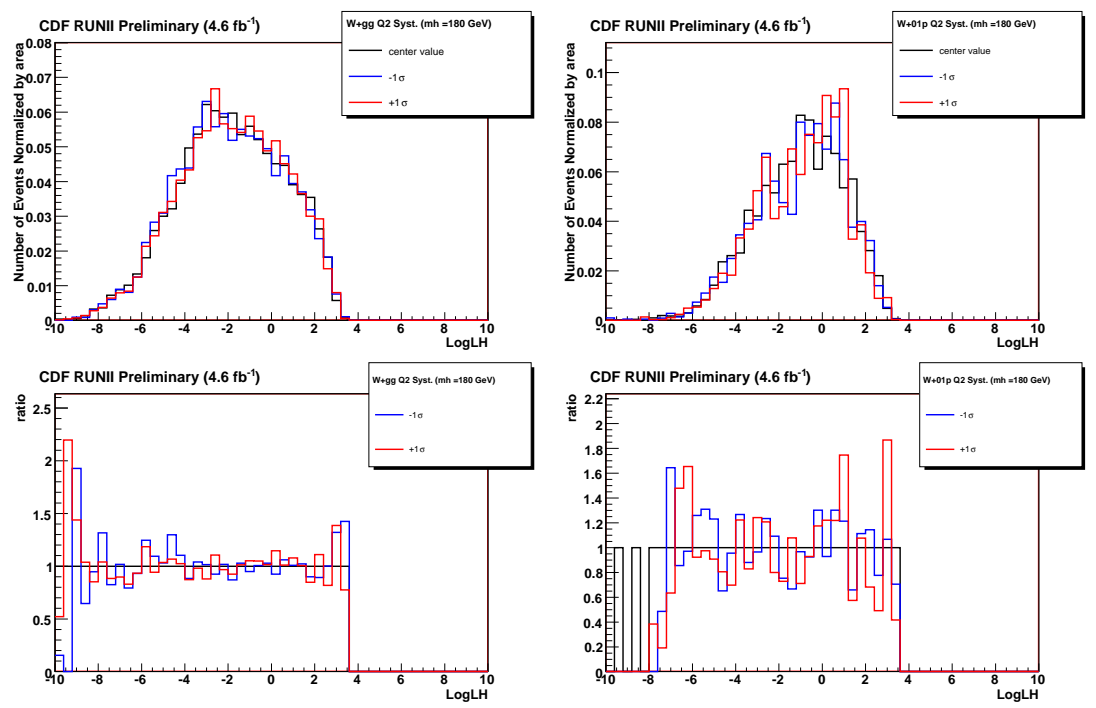

Figure 6.6: Likelihood output distribution using the $W+$ jets $\mathrm{Q}^{2}$ systematic samples at $m_{H}=$ $180 \mathrm{GeV} / \mathrm{c}^{2}$. The black line shows the center value. The red histogram shows the distribution for $+1 \sigma\left(2.0 \times \mathrm{Q}^{2}\right)$. The blue histogram shows the distribution for $-1 \sigma\left(0.5 \times \mathrm{Q}^{2}\right)$. The left top plot shows log likelihood output for $W+$ gg background. The left bottom plot shows ratios which are $\pm 1 \sigma /$ (center value). The right plots show log likelihood output for $W+0$ or 1 parton background. 



\section{Chapter 7}

\section{Results and Discussions}

\subsection{Upper Limits on Higgs Boson Production Cross Section}

There is no significant excess of events in the data comparing the background expectation and the likelihood distributions, Then, we fit the likelihood distribution and extract 95\% C.L. upper limits using pseudo-experiments based on the background expectation distribution.

We set an upper limit on the Higgs boson production cross section times branching ratio of $g g \rightarrow H \rightarrow W W^{(*)}$ as a function of $m_{H}$ by using the number of events in the $W+2$ jets sample. Since there are no peaks observed in the likelihood distribution, we assume that the $W+2$ jets and likelihood distributions in the data consist of $W+$ jets, Electroweak ( $W W, W Z, Z Z, t \bar{t}$ and single top), $Z+$ jets and non- $W$. A 1-dimensional binned maximum likelihood technique is used to obtain the $95 \%$ C.L. upper limit on the cross section of signal process. The expected number of events $\left(\mu_{i}\right)$ in each mass bin is

$$
\mu_{i}=\sum_{k=1}^{\mathrm{Bkg}} f_{i}^{k} \cdot N^{k}+f_{i}^{g g \rightarrow H} \cdot\left(\varepsilon \cdot \mathcal{L} \cdot \sigma_{g g \rightarrow H}\right),
$$

where k corresponds to background index for $W+$ jets, Electroweak, $Z+$ jets, and non- $W . f_{i}^{k}$ and $f_{i}^{g g \rightarrow H}$ are the expected fractions of events for the background and the signal in a given mass bin, predicted by MC. $N^{k}, \varepsilon, \mathcal{L}$ and $\sigma_{g g \rightarrow H}$ are the expected number of background events, the detection efficiency, the luminosity, and the unknown $g g \rightarrow H$ cross section, respectively. In pseudo-experiments, we select background events independently according to Gaussian distributions with the estimated total uncertainty as the standard deviations. The corresponding likelihood is then

$$
\begin{aligned}
L\left(\sigma / \sigma_{\mathrm{SM}}\right) & =\int \cdots \int \prod_{i \in \text { bins }} \frac{\mu_{i}^{N_{i}} \cdot e^{-\mu_{i}}}{N_{i} !} \prod_{k=1}^{B k g} G\left(N_{k}, \sigma_{N_{k}}\right) \\
& \times G\left(N_{g g \rightarrow H}, \sigma_{N_{g g \rightarrow H}}\right) d N_{k} d N_{g g \rightarrow H} .
\end{aligned}
$$

We define the expected limit by taking the median value of the pseudo-experiments.

Figure 7.1 shows the obtained upper limits.

\subsection{Discussion}

A search for the Standard Model Higgs boson in $H \rightarrow W W^{(*)} \rightarrow \ell \nu j j$ is also performed by the ATLAS experiment (Figure 7.2) at the Large Hadron Collider (LHC) at CERN. The cms energy 


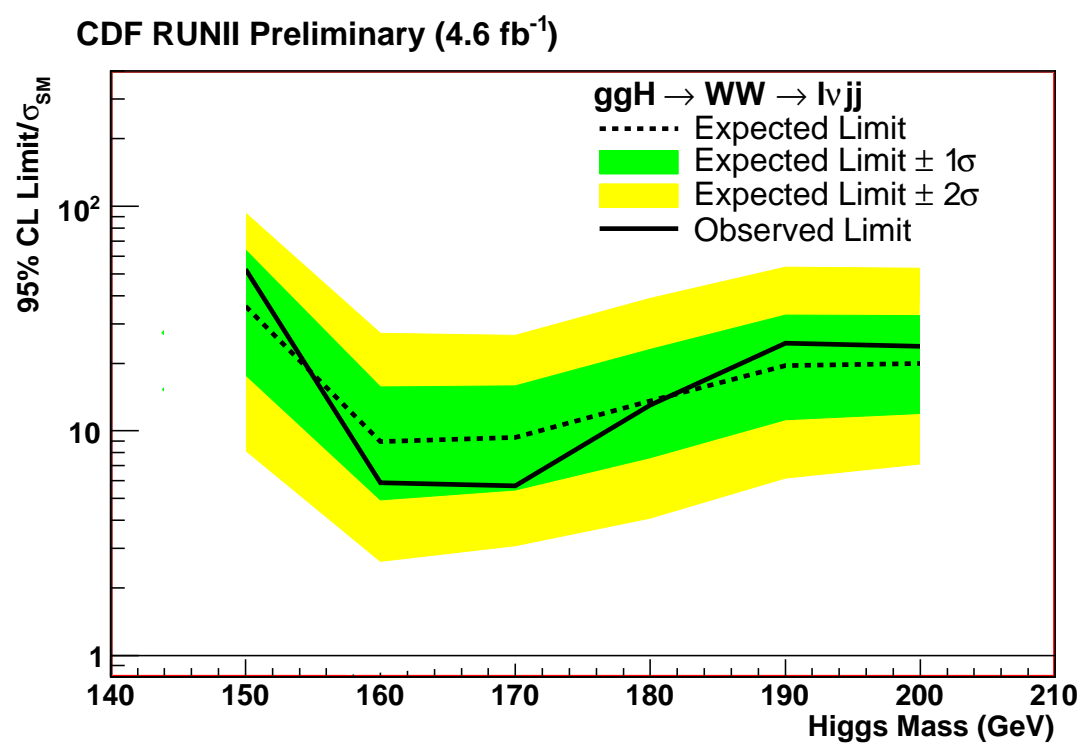

Figure 7.1: The dashed line and the solid line show the expected and observed upper limits on cross section normalized by SM Higgs boson cross section.

Table 7.1: Expected and observed 95\% C.L. upper limits on cross section for the Standard Model higgs boson in $H \rightarrow W W^{(*)} \rightarrow l \nu j j$ channel. The values are normalized by the Standard Model Higgs boson cross sections.

\begin{tabular}{ccccccc}
\multicolumn{2}{l}{ CDF RUNII Preliminary $\left(4.6 \mathrm{fb}^{-1}\right)$} \\
\hline \hline Higgs Mass $\left(\mathrm{GeV} / \mathrm{c}^{2}\right)$ & 150 & 160 & 170 & 180 & 190 & 200 \\
\hline$-2 \sigma$ & 8.17 & 2.62 & 3.08 & 4.08 & 6.15 & 7.11 \\
$-1 \sigma$ & 17.7 & 4.93 & 5.44 & 7.61 & 11.2 & 11.9 \\
median U.L./ $\sigma_{\mathrm{SM}}$ & 35.9 & 8.97 & 9.36 & 13.6 & 19.5 & 19.9 \\
$+1 \sigma$ & 63.8 & 15.6 & 15.9 & 23.1 & 32.7 & 32.5 \\
$+2 \sigma$ & 93.2 & 27.2 & 26.6 & 38.9 & 53.6 & 52.9 \\
\hline Obs. U.L./ $\sigma_{\mathrm{SM}}$ & 52.5 & 5.88 & 5.69 & 13.0 & 24.5 & 23.8 \\
\hline \hline
\end{tabular}


of LHC is $7 \mathrm{TeV}$. Then, Higgs boson production cross section is several times higher than cross section at Tevatron. The luminosity of LHC is also higher than Tevatron.

However, they have huge QCD backgrounds because of a higher cms energy. This huge QCD backgrounds prevent their Higgs boson search in $H \rightarrow W W^{(*)} \rightarrow \ell \nu j j$ channel ranging $m_{H}$ below $200 \mathrm{GeV} / \mathrm{c}^{2}$.

Then CDF and D0 are only two experiment which can search for the Standard Model Higgs boson in $H \rightarrow W W^{(*)} \rightarrow \ell \nu j j$ channel ranging $m_{H}$ below $200 \mathrm{GeV} / \mathrm{c}^{2}$. This analysis can add $\sim 1 \%$ sensitivity to the Tevatron combined upper limits on cross section for SM Higgs boson.

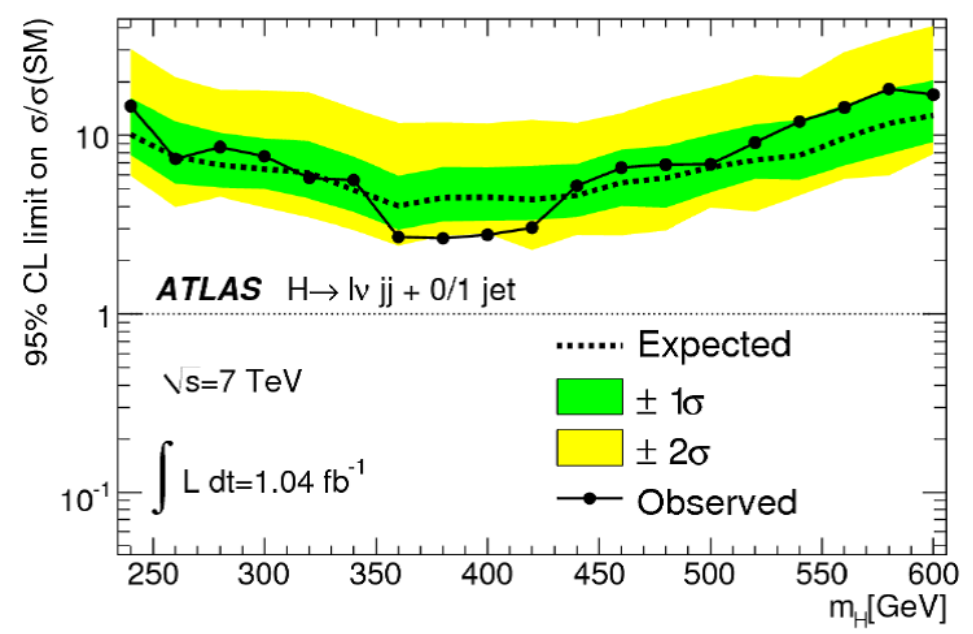

Figure 7.2: Result of $H \rightarrow \ell \nu j j$ search at ATLAS experiment. 



\section{Chapter 8}

\section{Conclusions}

We have presented the results of a search for the Standard Model higgs boson in $H \rightarrow W W^{(*)} \rightarrow l \nu j j$ channel with $4.6 \mathrm{fb}^{-1}$ of data collected With the CDF detector Tevatron RUN II. We have not observed any significant excess of events over the known background in the likelihood distribution. Therefore, we have set upper limits on the cross section times the branching ratio $\sigma_{S M}(g g \rightarrow H) \times$ $\operatorname{Br}\left(H \rightarrow W W^{(*)}\right)$ using the likelihood distribution. We set upper limits on Higgs boson production cross section ranging from $5.69 \times \sigma_{\mathrm{SM}}\left(m_{H}=170 \mathrm{GeV} / c^{2}\right)$ to $52.5 \times \sigma_{\mathrm{SM}}\left(m_{H}=\right.$ $150 \mathrm{GeV} / c^{2}$ ) at $95 \%$ confidence level. 



\section{Appendix A}

\section{Signal and Background Templates}

Figure A.1, A.2 and A. 3 show the signal and background templates at $\mathrm{m}_{H}=150 \mathrm{GeV}$. Figure A.4, A.5 and A.6 show the signal and background templates at $\mathrm{m}_{H}=160 \mathrm{GeV}$. Figure A.7, A.8 and A.9 show the signal and background templates at $\mathrm{m}_{H}=170 \mathrm{GeV}$. Figure A.10, A.11 and A.12 show the signal and background templates at $\mathrm{m}_{H}=180 \mathrm{GeV}$. Figure A.13, A.14 and A.15 show the signal and background templates at $\mathrm{m}_{H}=190 \mathrm{GeV}$. Figure A.16, A.17 and A.18 show the signal and background templates at $\mathrm{m}_{H}=200 \mathrm{GeV}$.
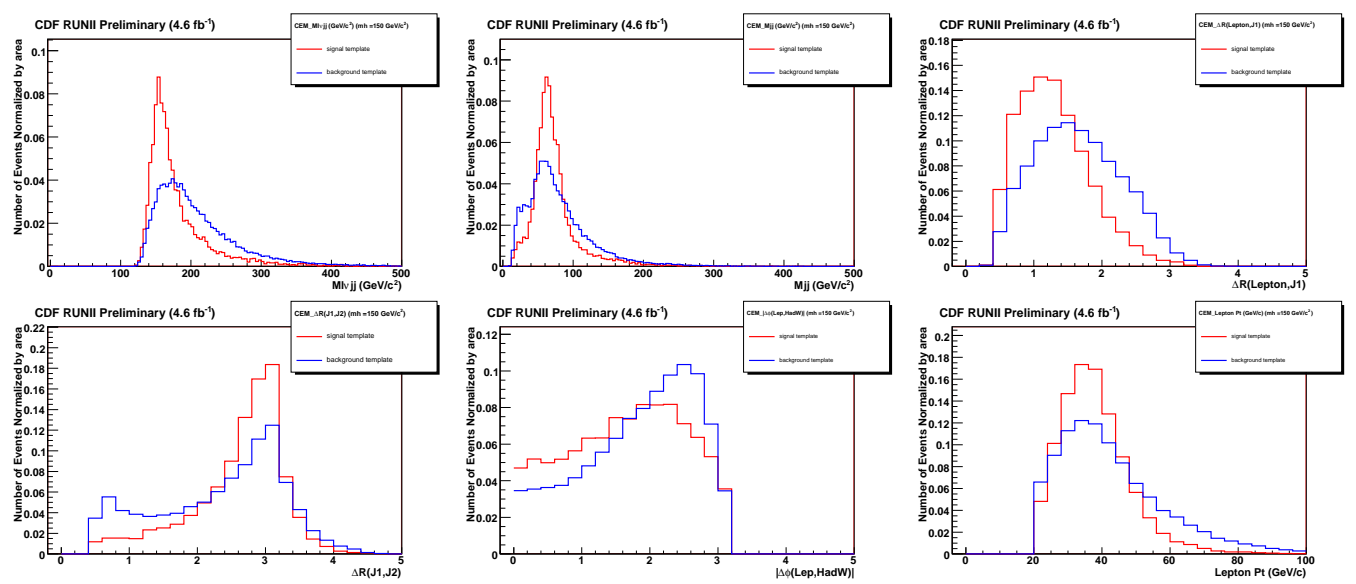

Figure A.1: Likelihood templates for the CEM. These histograms are (Red) $H \rightarrow W W$, $m_{H}=150 \mathrm{GeV} / \mathrm{c}^{2}$, and (Blue)background. Top column from left: reconstructed higgs mass, dijet mass, $\Delta R$ (lepton, Down-type jet). Bottom from left: $\Delta R$ (up-type jet, down-type jet), $\mid \Delta \phi\left(\right.$ lepton,$\left.W_{\text {hadronic }}\right) \mid, P_{T}^{\text {lepton }}$. 

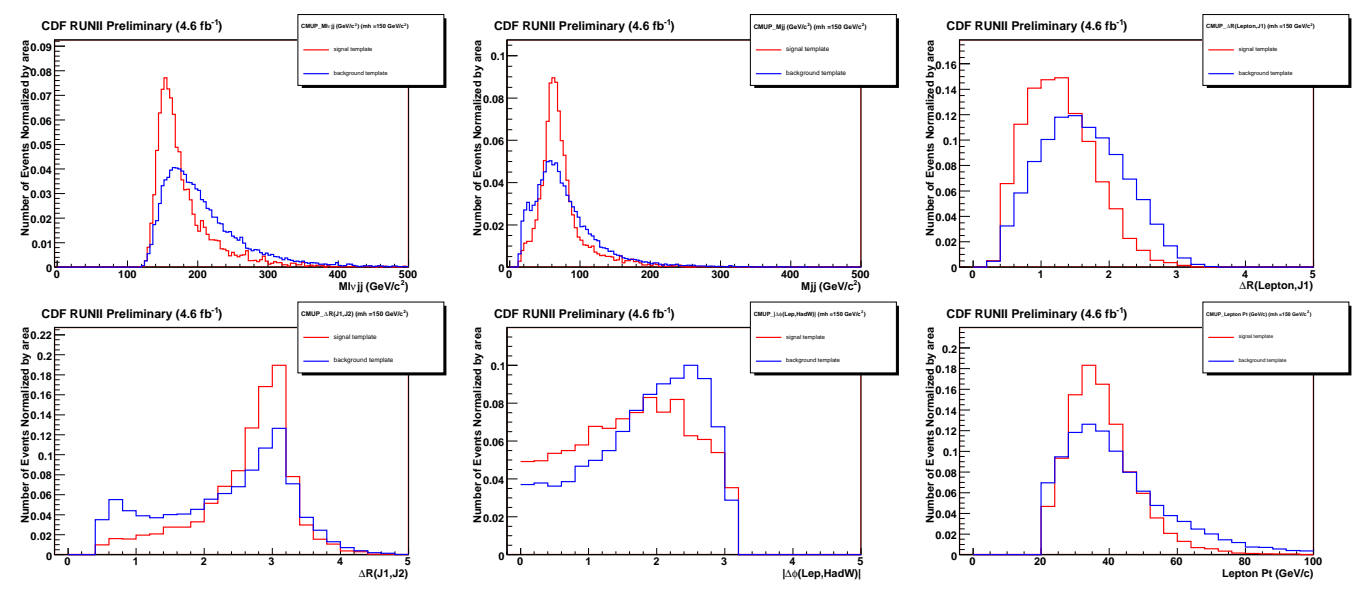

Figure A.2: Likelihood templates for the CMUP. These histograms are (Red) $H \rightarrow W W$, $m_{H}=150 \mathrm{GeV} / \mathrm{c}^{2}$, and (Blue)background. Top column from left: reconstructed higgs mass, dijet mass, $\Delta R$ (lepton, Down-type jet). Bottom from left: $\Delta R$ (up-type jet, down-type jet), $\mid \Delta \phi\left(\right.$ lepton, $\left.W_{\text {hadronic }}\right) \mid, P_{T}^{\text {lepton }}$.
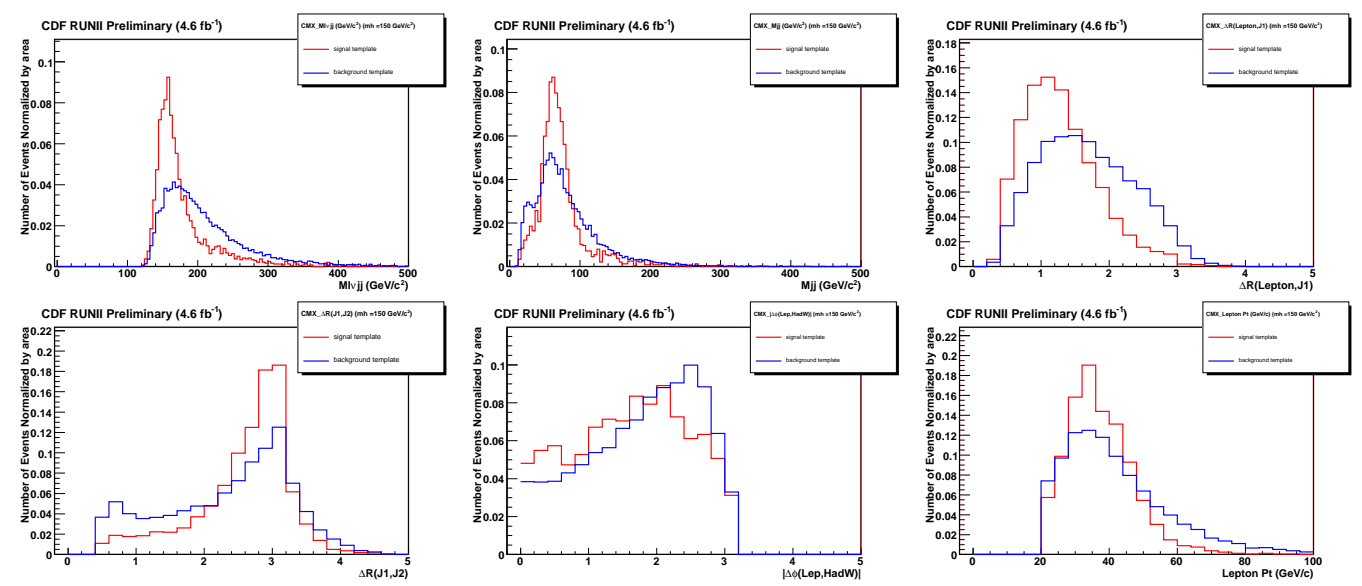

Figure A.3: Likelihood templates for the CMX. These histograms are (Red) $H \rightarrow W W$, $m_{H}=150 \mathrm{GeV} / \mathrm{c}^{2}$, and (Blue)background. Top column from left: reconstructed higgs mass, dijet mass, $\Delta R$ (lepton, Down-type jet). Bottom from left: $\Delta R$ (up-type jet, down-type jet), $\mid \Delta \phi\left(\right.$ lepton,$\left.W_{\text {hadronic }}\right) \mid, P_{T}^{\text {lepton }}$. 

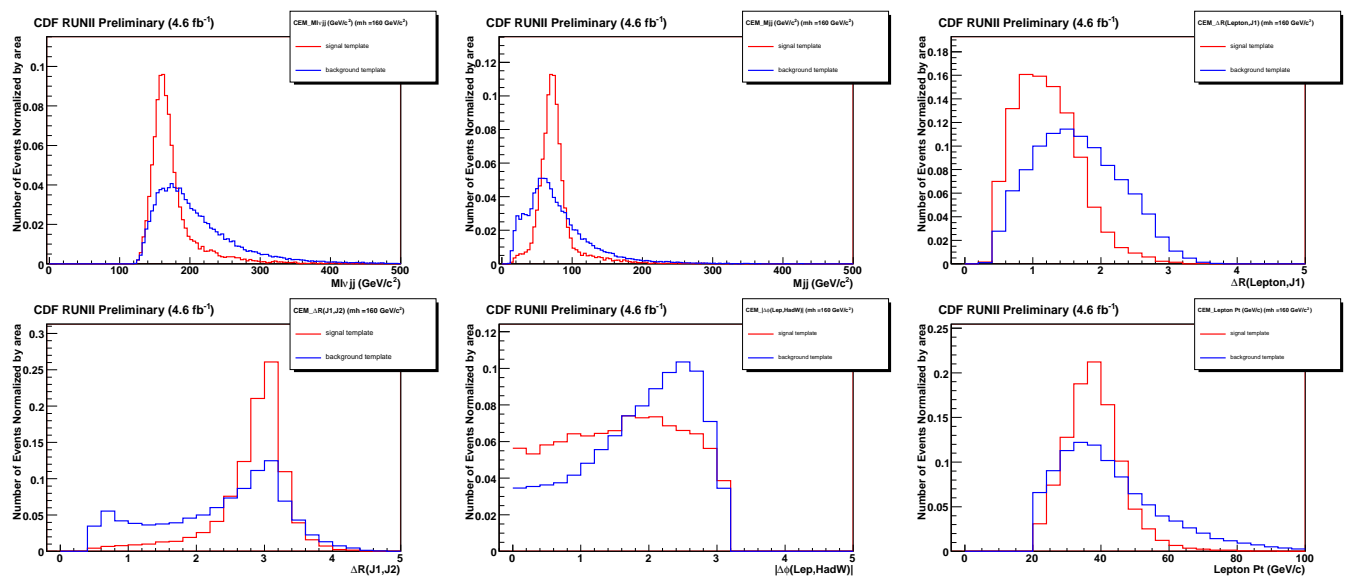

Figure A.4: Likelihood templates for the CEM. These histograms are (Red) $H \rightarrow W W$, $m_{H}=160 \mathrm{GeV} / \mathrm{c}^{2}$, and (Blue)background. Top column from left: reconstructed higgs mass, dijet mass, $\Delta R$ (lepton, Down-type jet). Bottom from left: $\Delta R$ (up-type jet, down-type jet), $\mid \Delta \phi\left(\right.$ lepton,$\left.W_{\text {hadronic }}\right) \mid, P_{T}^{\text {lepton }}$. 

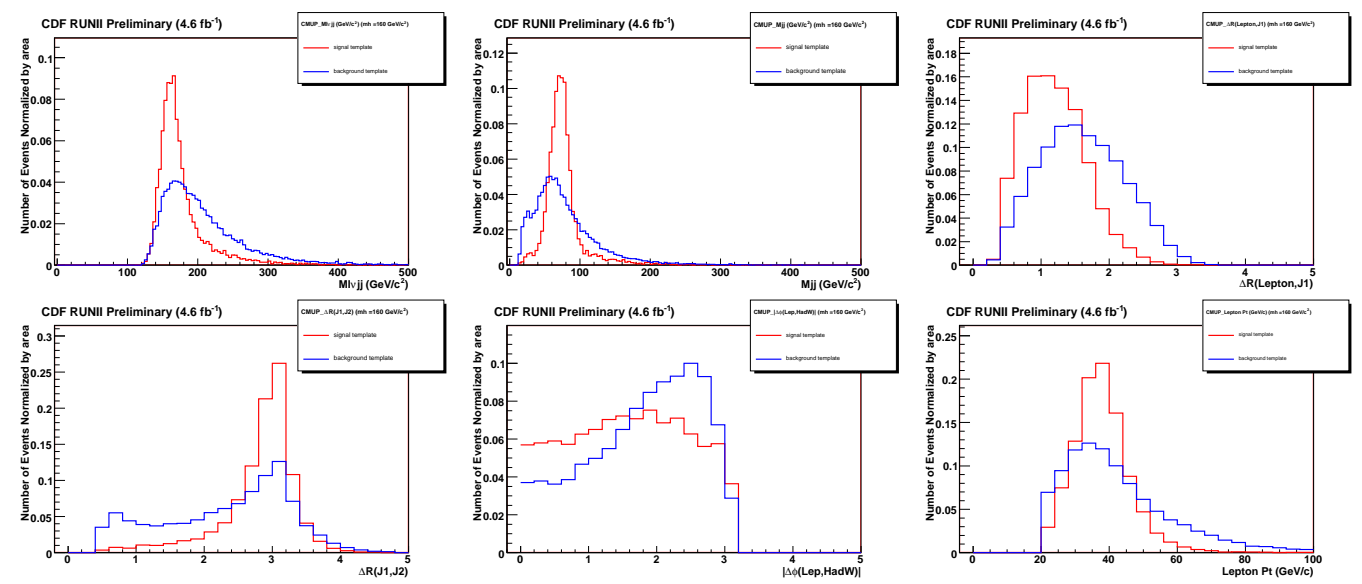

Figure A.5: Likelihood templates for the CMUP. These histograms are (Red) $H \rightarrow W W$, $m_{H}=160 \mathrm{GeV} / \mathrm{c}^{2}$, and (Blue)background. Top column from left: reconstructed higgs mass, dijet mass, $\Delta R$ (lepton, Down-type jet). Bottom from left: $\Delta R$ (up-type jet, down-type jet), $\mid \Delta \phi\left(\right.$ lepton, $\left.W_{\text {hadronic }}\right) \mid, P_{T}^{\text {lepton }}$.
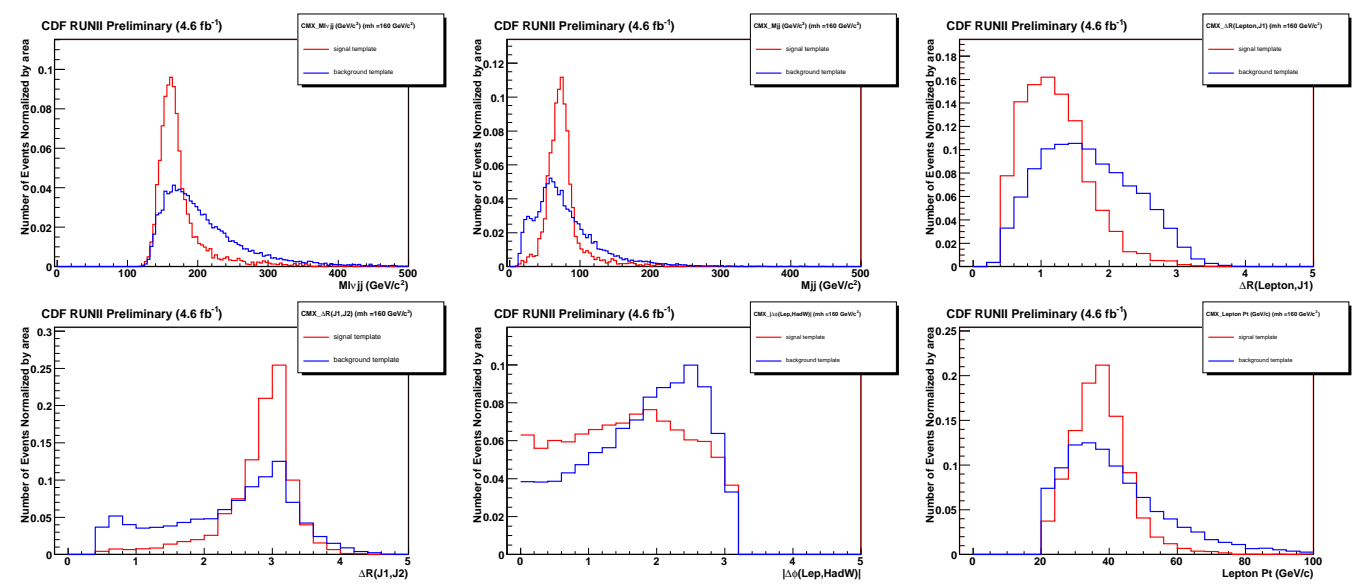

Figure A.6: Likelihood templates for the CMX. These histograms are (Red) $H \rightarrow W W$, $m_{H}=160 \mathrm{GeV} / \mathrm{c}^{2}$, and (Blue)background. Top column from left: reconstructed higgs mass, dijet mass, $\Delta R$ (lepton, Down-type jet). Bottom from left: $\Delta R$ (up-type jet, down-type jet), $\mid \Delta \phi\left(\right.$ lepton,$\left.W_{\text {hadronic }}\right) \mid, P_{T}^{\text {lepton }}$. 

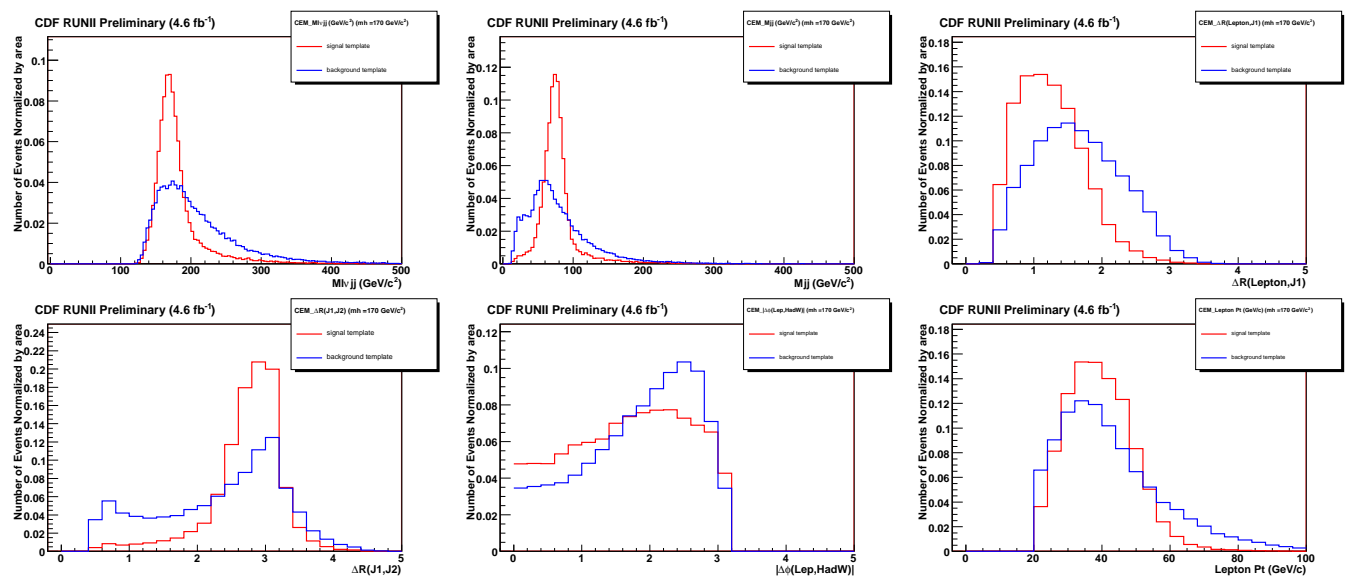

Figure A.7: Likelihood templates for the CEM. These histograms are (Red) $H \rightarrow W W$, $m_{H}=170 \mathrm{GeV} / \mathrm{c}^{2}$, and (Blue)background. Top column from left: reconstructed higgs mass, dijet mass, $\Delta R$ (lepton, Down-type jet). Bottom from left: $\Delta R$ (up-type jet, down-type jet), $\mid \Delta \phi\left(\right.$ lepton,$\left.W_{\text {hadronic }}\right) \mid, P_{T}^{\text {lepton }}$. 

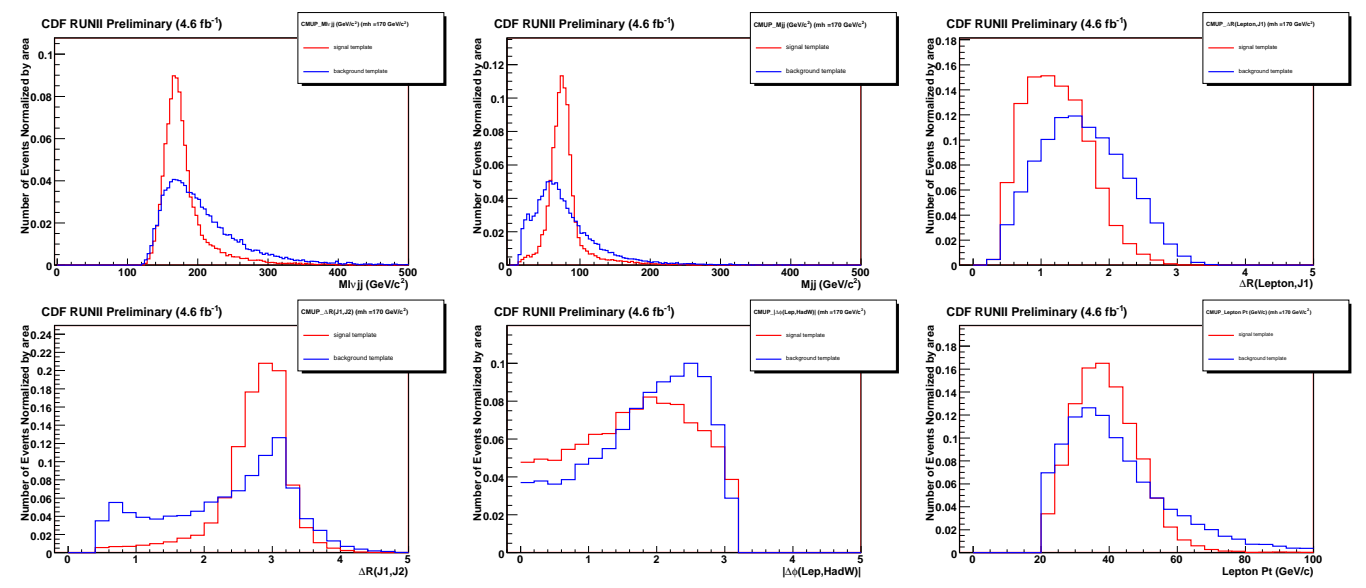

Figure A.8: Likelihood templates for the CMUP. These histograms are (Red) $H \rightarrow W W$, $m_{H}=170 \mathrm{GeV} / \mathrm{c}^{2}$, and (Blue)background. Top column from left: reconstructed higgs mass, dijet mass, $\Delta R$ (lepton, Down-type jet). Bottom from left: $\Delta R$ (up-type jet, down-type jet), $\mid \Delta \phi\left(\right.$ lepton, $\left.W_{\text {hadronic }}\right) \mid, P_{T}^{\text {lepton }}$.
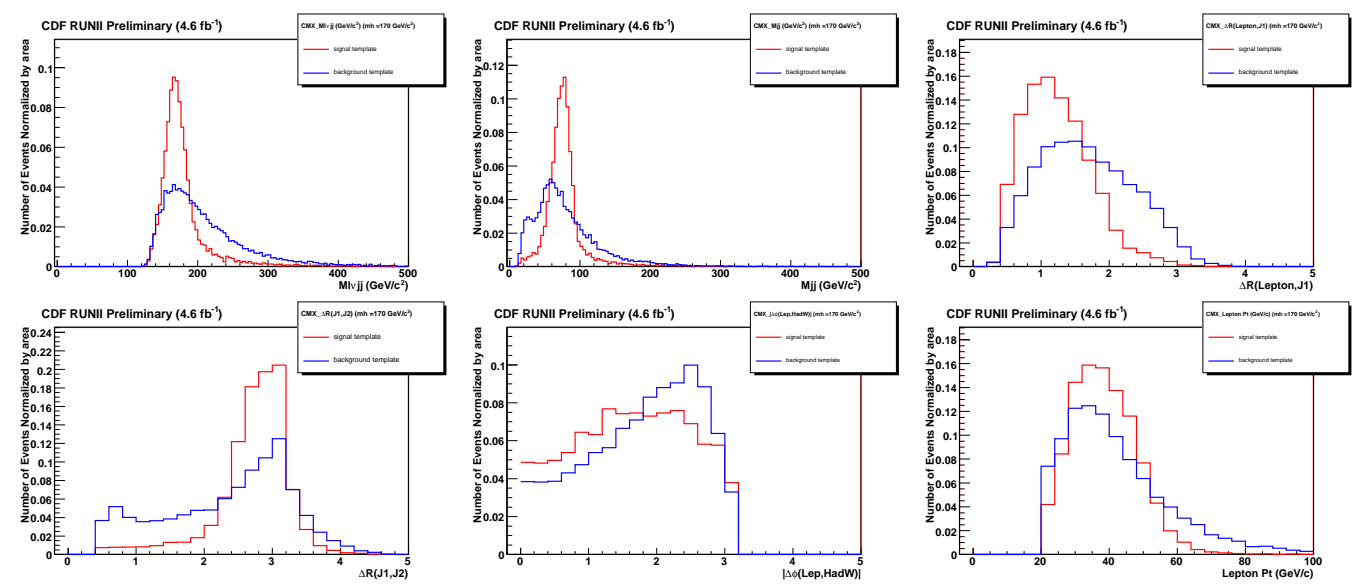

Figure A.9: Likelihood templates for the CMX. These histograms are (Red) $H \rightarrow W W$, $m_{H}=170 \mathrm{GeV} / \mathrm{c}^{2}$, and (Blue)background. Top column from left: reconstructed higgs mass, dijet mass, $\Delta R$ (lepton, Down-type jet). Bottom from left: $\Delta R$ (up-type jet, down-type jet), $\mid \Delta \phi\left(\right.$ lepton,$\left.W_{\text {hadronic }}\right) \mid, P_{T}^{\text {lepton }}$. 

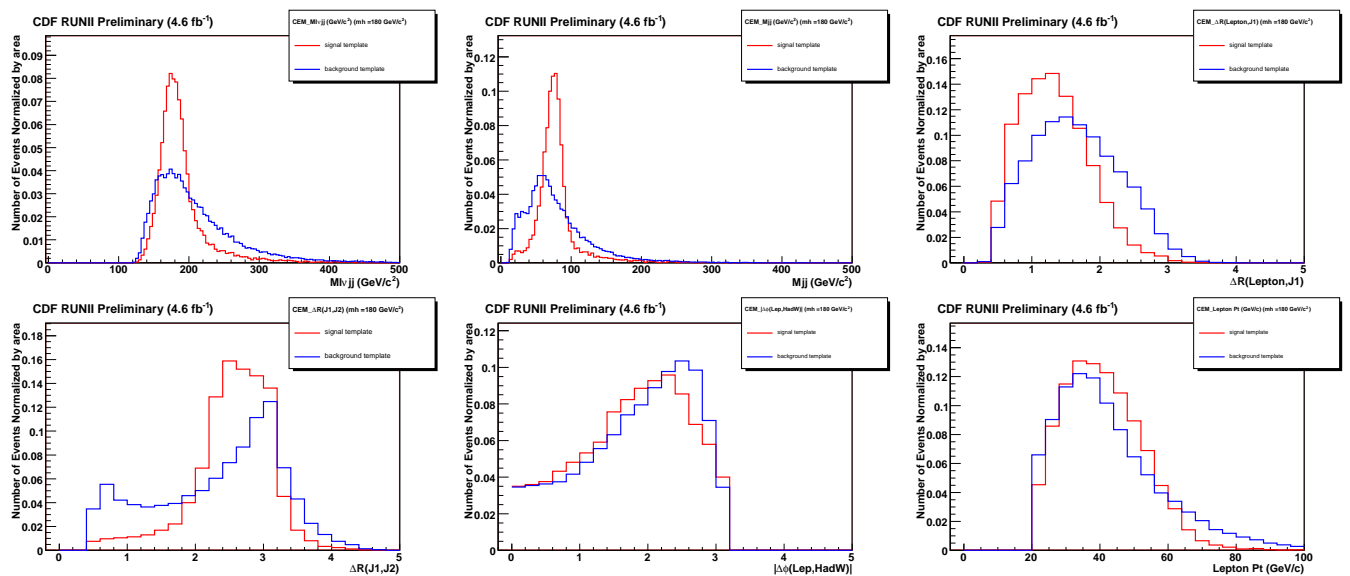

Figure A.10: Likelihood templates for the CEM. These histograms are (Red) $H \rightarrow W W$, $m_{H}=180 \mathrm{GeV} / \mathrm{c}^{2}$, and (Blue)background. Top column from left: reconstructed higgs mass, dijet mass, $\Delta R$ (lepton, Down-type jet). Bottom from left: $\Delta R$ (up-type jet, down-type jet), $\mid \Delta \phi\left(\right.$ lepton,$\left.W_{\text {hadronic }}\right) \mid, P_{T}^{\text {lepton }}$. 

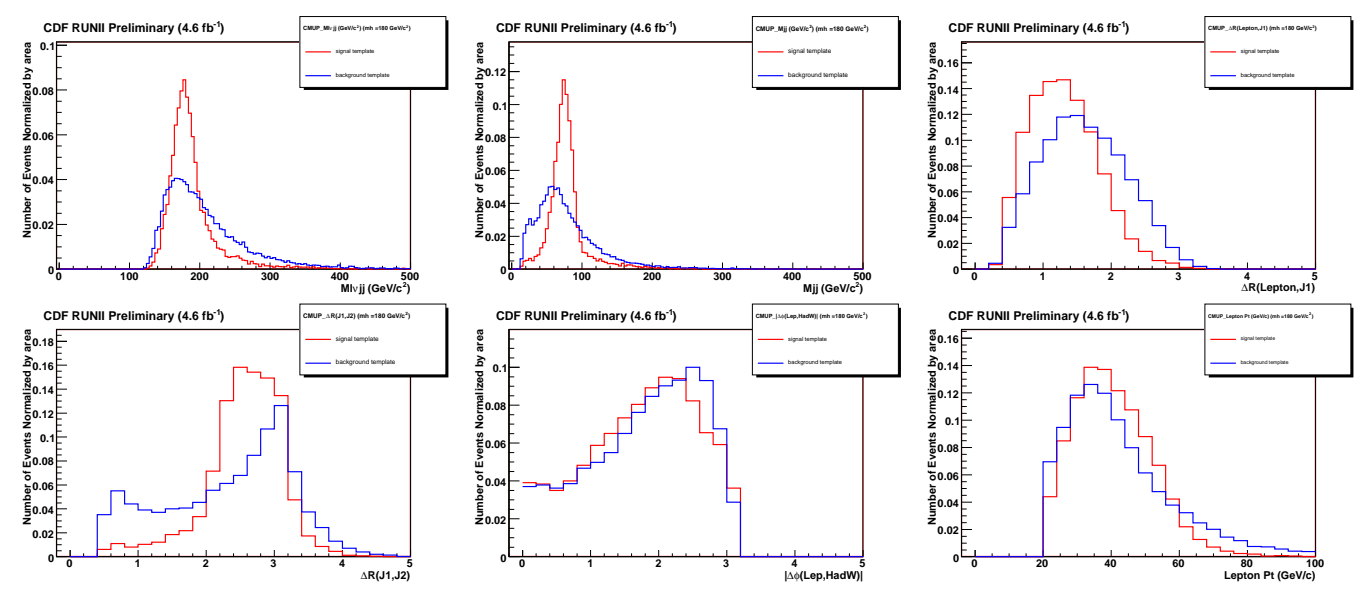

Figure A.11: Likelihood templates for the CMUP. These histograms are (Red) $H \rightarrow W W$, $m_{H}=180 \mathrm{GeV} / \mathrm{c}^{2}$, and (Blue)background. Top column from left: reconstructed higgs mass, dijet mass, $\Delta R$ (lepton, Down-type jet). Bottom from left: $\Delta R$ (up-type jet, down-type jet), $\mid \Delta \phi\left(\right.$ lepton, $\left.W_{\text {hadronic }}\right) \mid, P_{T}^{\text {lepton }}$.
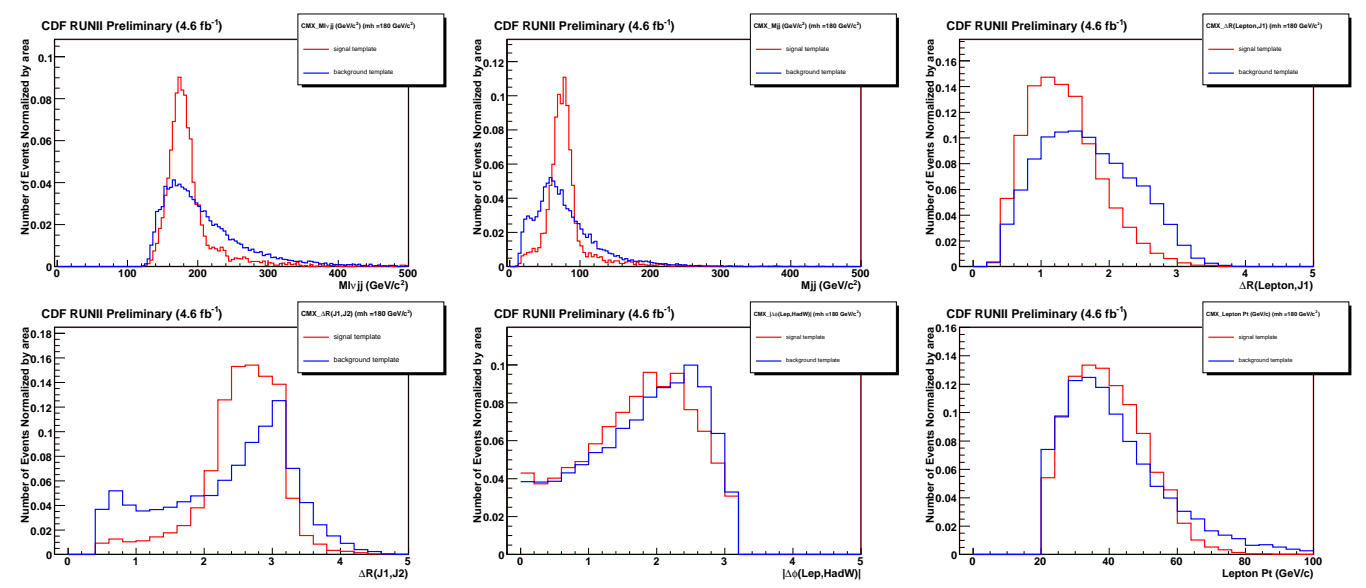

Figure A.12: Likelihood templates for the CMX. These histograms are (Red) $H \rightarrow W W$, $m_{H}=180 \mathrm{GeV} / \mathrm{c}^{2}$, and (Blue)background. Top column from left: reconstructed higgs mass, dijet mass, $\Delta R$ (lepton, Down-type jet). Bottom from left: $\Delta R$ (up-type jet, down-type jet), $\mid \Delta \phi\left(\right.$ lepton,$\left.W_{\text {hadronic }}\right) \mid, P_{T}^{\text {lepton }}$. 

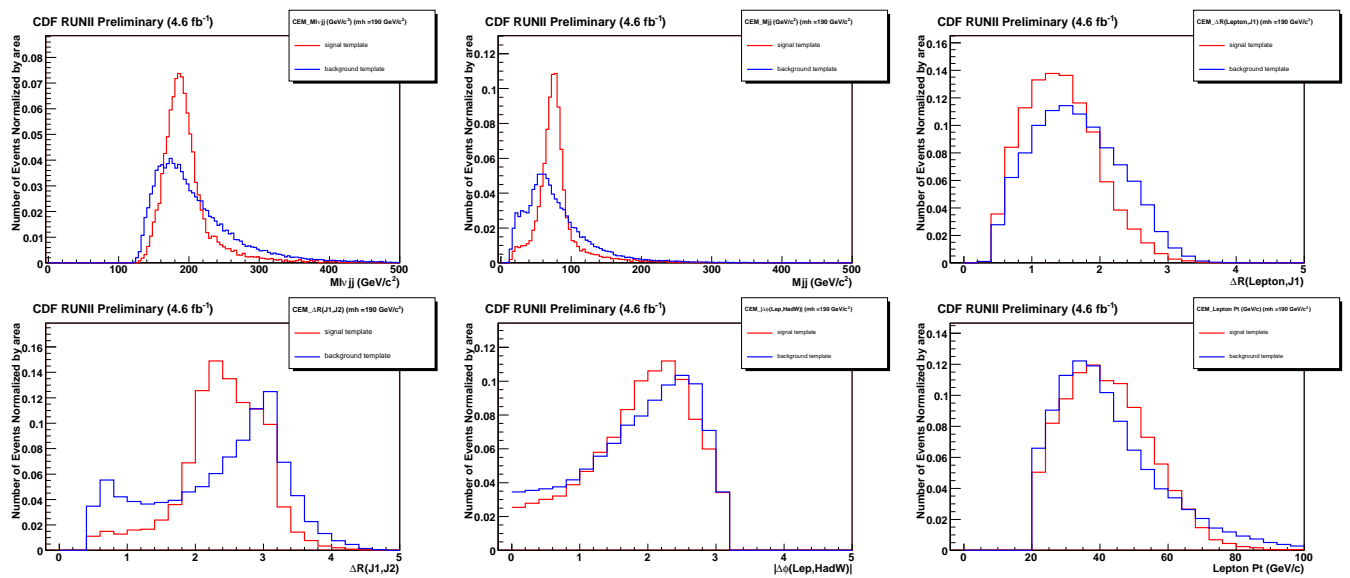

Figure A.13: Likelihood templates for the CEM. These histograms are (Red) $H \rightarrow W W$, $m_{H}=190 \mathrm{GeV} / \mathrm{c}^{2}$, and (Blue)background. Top column from left: reconstructed higgs mass, dijet mass, $\Delta R$ (lepton, Down-type jet). Bottom from left: $\Delta R$ (up-type jet, down-type jet), $\mid \Delta \phi\left(\right.$ lepton,$\left.W_{\text {hadronic }}\right) \mid, P_{T}^{\text {lepton }}$. 

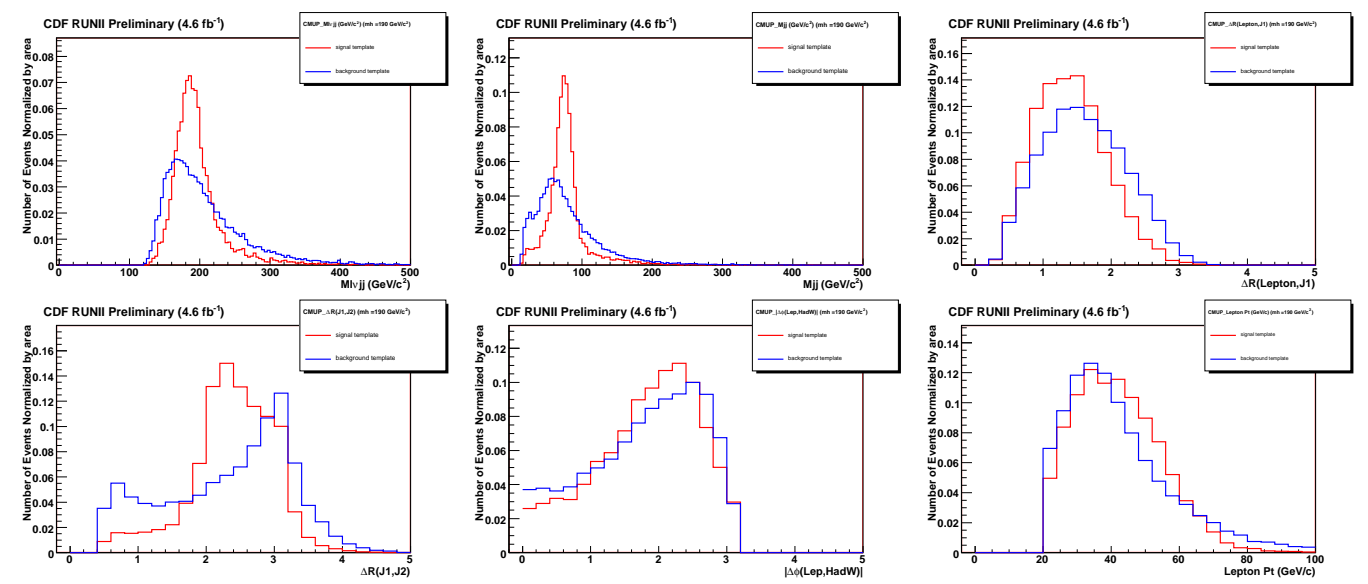

Figure A.14: Likelihood templates for the CMUP. These histograms are (Red) $H \rightarrow W W$, $m_{H}=190 \mathrm{GeV} / \mathrm{c}^{2}$, and (Blue)background. Top column from left: reconstructed higgs mass, dijet mass, $\Delta R$ (lepton, Down-type jet). Bottom from left: $\Delta R$ (up-type jet, down-type jet), $\mid \Delta \phi\left(\right.$ lepton, $\left.W_{\text {hadronic }}\right) \mid, P_{T}^{\text {lepton }}$.
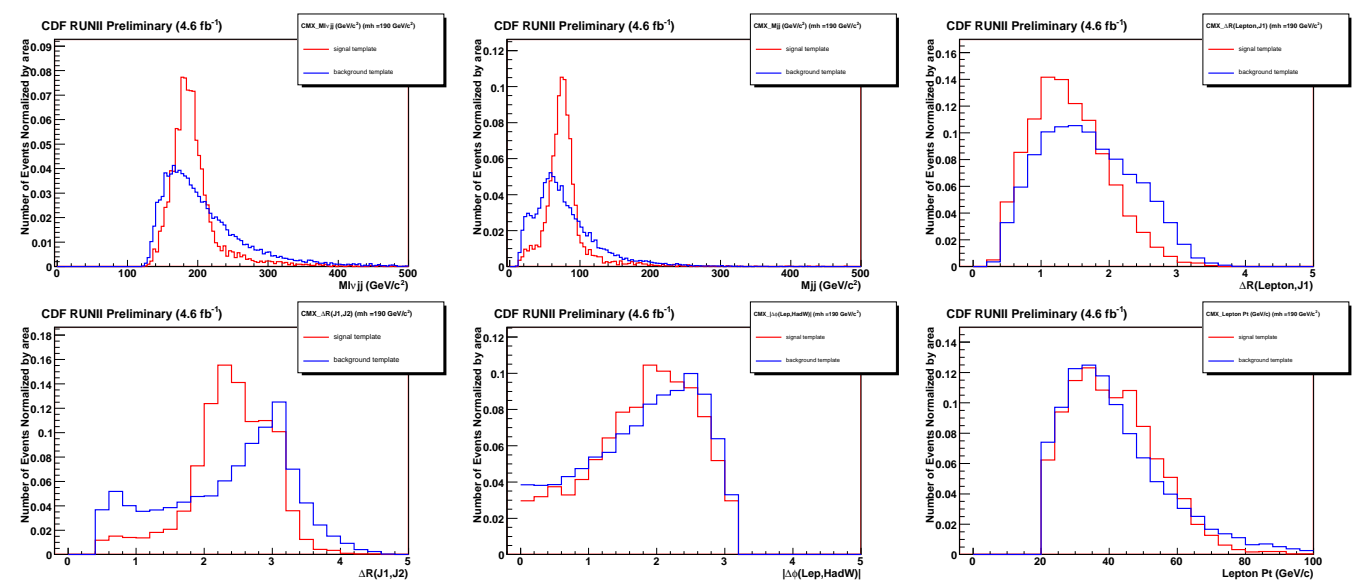

Figure A.15: Likelihood templates for the CMX. These histograms are (Red) $H \rightarrow W W$, $m_{H}=190 \mathrm{GeV} / \mathrm{c}^{2}$, and (Blue)background. Top column from left: reconstructed higgs mass, dijet mass, $\Delta R$ (lepton, Down-type jet). Bottom from left: $\Delta R$ (up-type jet, down-type jet), $\mid \Delta \phi\left(\right.$ lepton,$\left.W_{\text {hadronic }}\right) \mid, P_{T}^{\text {lepton }}$. 

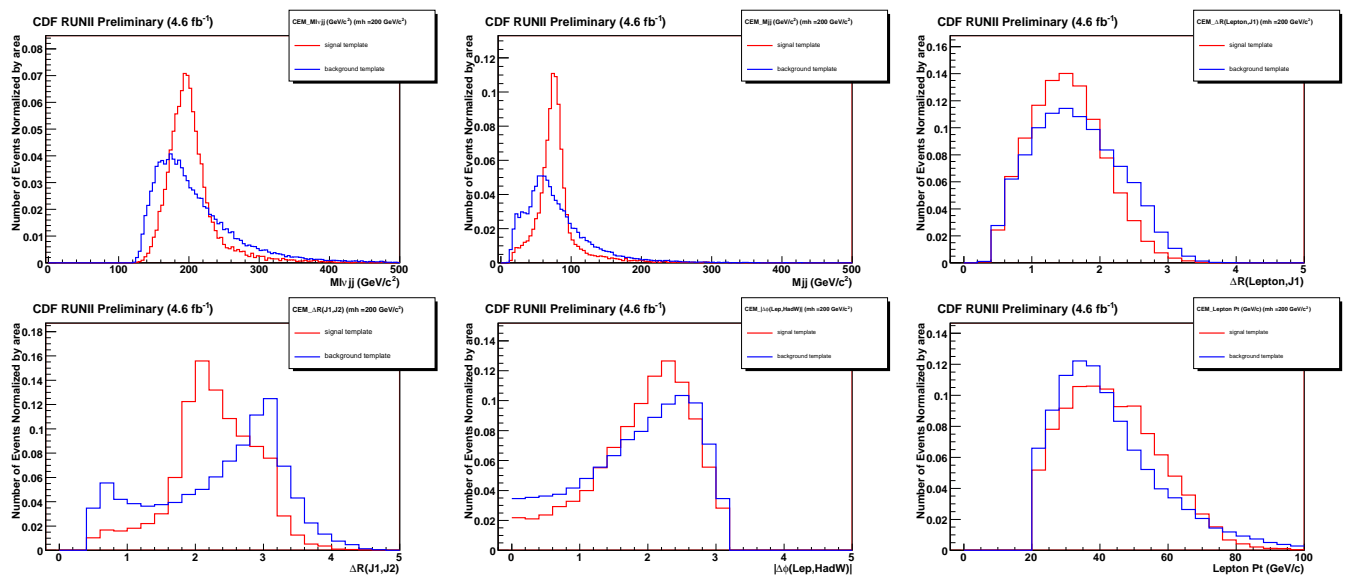

Figure A.16: Likelihood templates for the CEM. These histograms are (Red) $H \rightarrow W W$, $m_{H}=200 \mathrm{GeV} / \mathrm{c}^{2}$, and (Blue)background. Top column from left: reconstructed higgs mass, dijet mass, $\Delta R$ (lepton, Down-type jet). Bottom from left: $\Delta R$ (up-type jet, down-type jet), $\mid \Delta \phi\left(\right.$ lepton,$\left.W_{\text {hadronic }}\right) \mid, P_{T}^{\text {lepton }}$. 

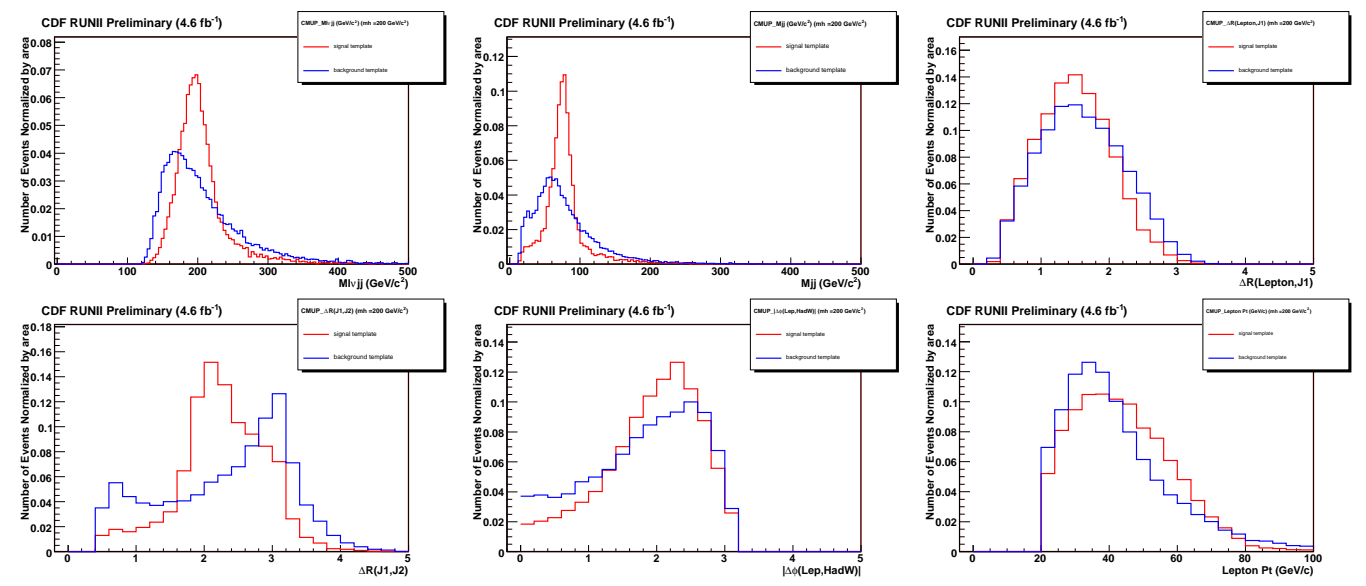

Figure A.17: Likelihood templates for the CMUP. These histograms are (Red) $H \rightarrow W W$, $m_{H}=200 \mathrm{GeV} / \mathrm{c}^{2}$, and (Blue)background. Top column from left: reconstructed higgs mass, dijet mass, $\Delta R$ (lepton, Down-type jet). Bottom from left: $\Delta R$ (up-type jet, down-type jet), $\mid \Delta \phi\left(\right.$ lepton, $\left.W_{\text {hadronic }}\right) \mid, P_{T}^{\text {lepton }}$.
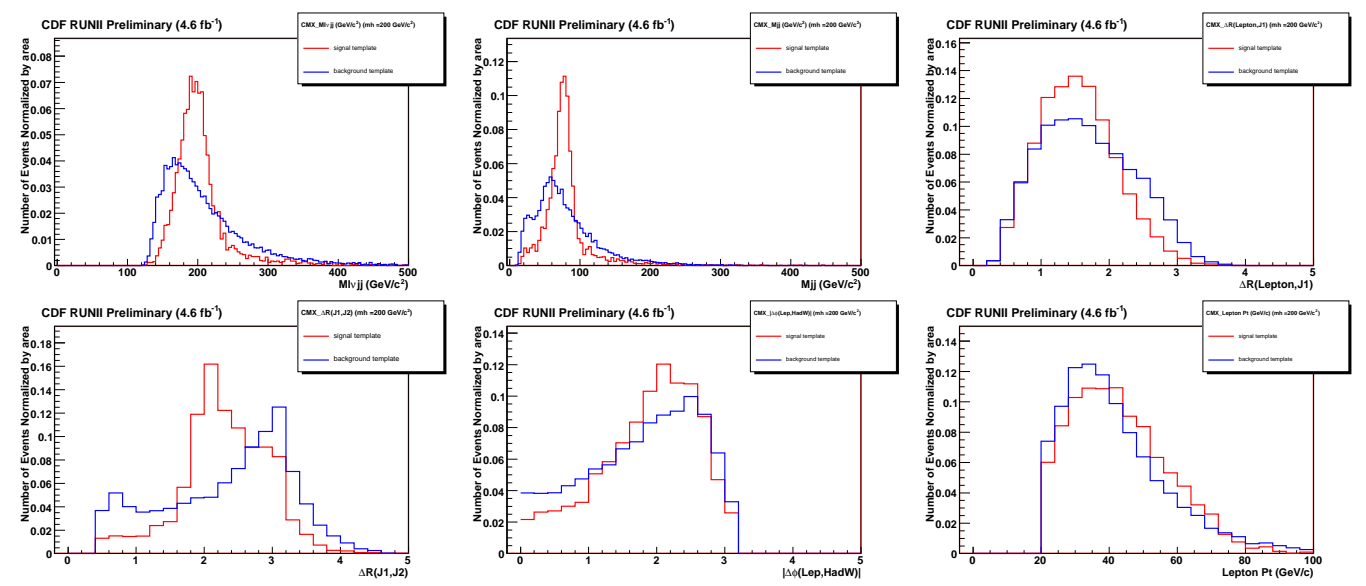

Figure A.18: Likelihood templates for the CMX. These histograms are (Red) $H \rightarrow W W$, $m_{H}=200 \mathrm{GeV} / \mathrm{c}^{2}$, and (Blue)background. Top column from left: reconstructed higgs mass, dijet mass, $\Delta R$ (lepton, Down-type jet). Bottom from left: $\Delta R$ (up-type jet, down-type jet), $\mid \Delta \phi\left(\right.$ lepton,$\left.W_{\text {hadronic }}\right) \mid, P_{T}^{\text {lepton }}$. 


\section{Appendix B}

\section{Input Variables and Output of the Likelihood}

Figure B.1, B.3, B.5, B.7, B.9 and B.11 show distributions of the input variables to the likelihood for $\mathrm{m}_{H}=150$ to $200 \mathrm{GeV} / \mathrm{c}^{2}$ in our final iteration. The background estimation is plotted along with data. Distribution of signal is also shown. On figures of likelihood input, Top column from left: reconstructed higgs mass, dijet mass, $\Delta R$ (lepton, Down-type jet). Bottom from left: $\Delta R$ (up-type jet, down-type jet), $\mid \Delta \phi\left(\right.$ lepton, $\left.W_{\text {hadronic }}\right) \mid, P_{T}^{\text {lepton }}$. The crosses shows the data, filled histograms are for backgrounds.

Figure B.2, B.4, B.6, B.8, B.10, B.12 shows the output of the likelihood for $\mathrm{m}_{H}=150$ to 200 $\mathrm{GeV} / \mathrm{c}^{2}$ in our final iteration.
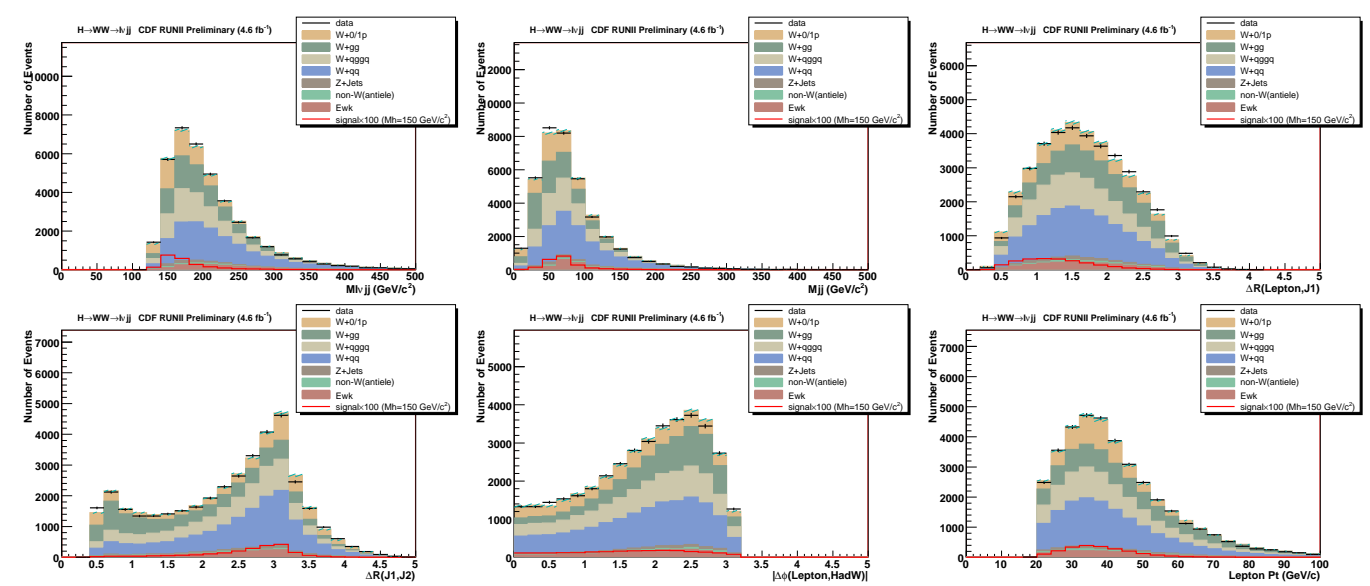

Figure B.1: Likelihood input distributions for $m_{H}=150 \mathrm{GeV} / \mathrm{c}^{2}$. The open red histogram shows the distribution for signal with $m_{H}=150 \mathrm{GeV} / \mathrm{c}^{2}$, scaled by a factor of 100 . 


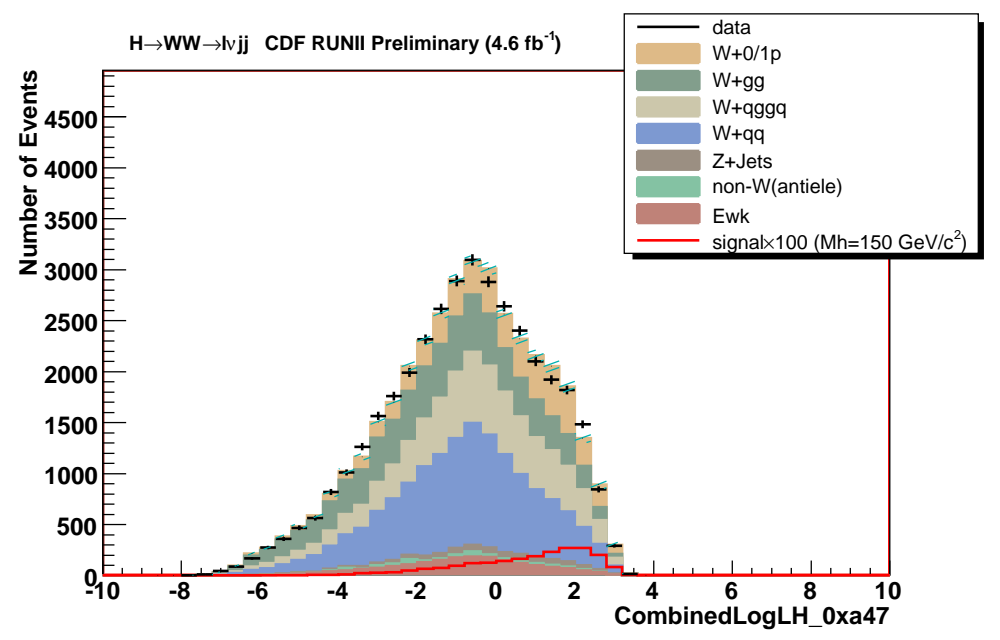

Figure B.2: Likelihood output distribution for $m_{H}=150 \mathrm{GeV} / \mathrm{c}^{2}$. The open red histogram shows the distribution for signal with $m_{H}=150 \mathrm{GeV} / \mathrm{c}^{2}$, scaled by a factor of 100 . 


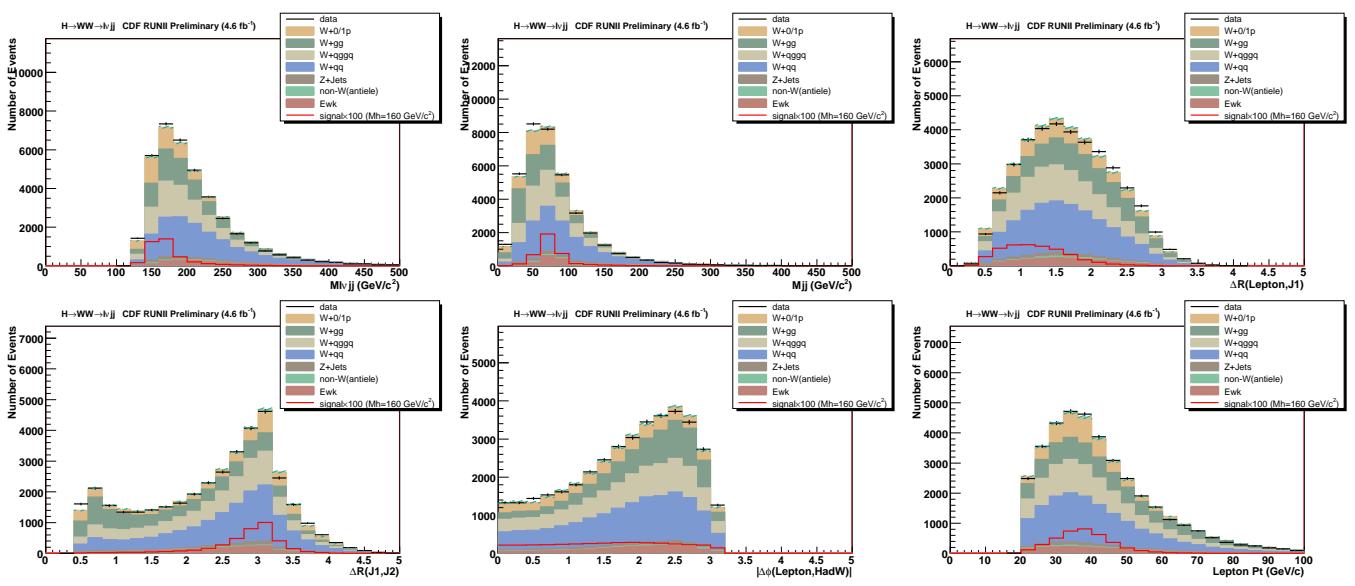

Figure B.3: Likelihood input distributions for $m_{H}=160 \mathrm{GeV} / \mathrm{c}^{2}$. The open red histogram shows the distribution for signal with $m_{H}=160 \mathrm{GeV} / \mathrm{c}^{2}$, scaled by a factor of 100 .

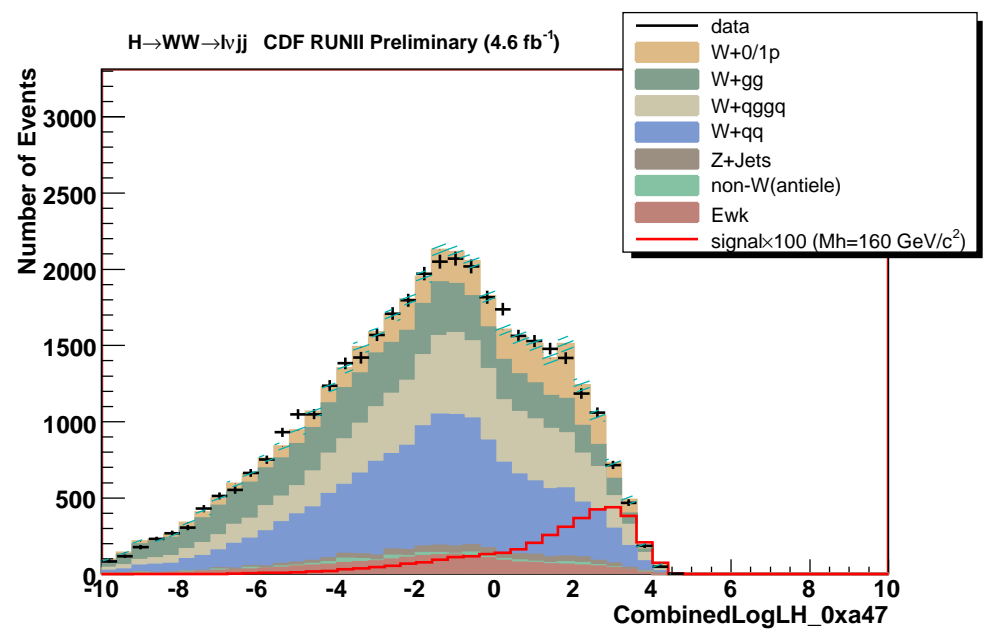

Figure B.4: Likelihood output distribution for $m_{H}=160 \mathrm{GeV} / \mathrm{c}^{2}$. The open red histogram shows the distribution for signal with $m_{H}=160 \mathrm{GeV} / \mathrm{c}^{2}$, scaled by a factor of 100 . 

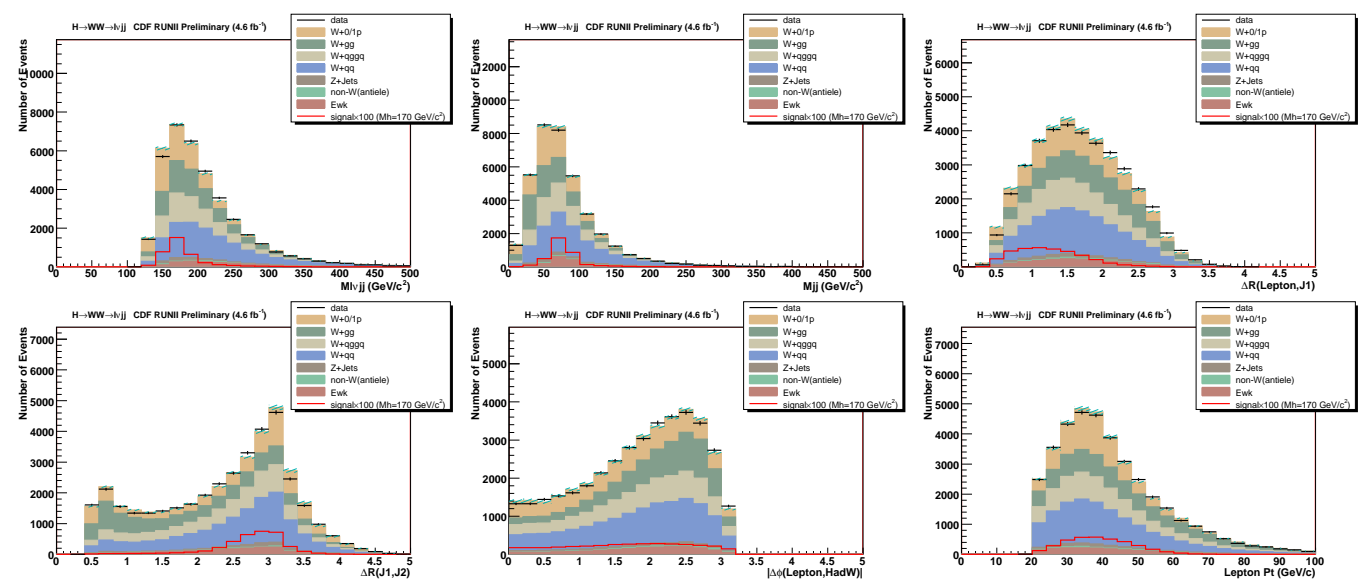

Figure B.5: Likelihood input distributions for $m_{H}=170 \mathrm{GeV} / \mathrm{c}^{2}$. The open red histogram shows the distribution for signal with $m_{H}=170 \mathrm{GeV} / \mathrm{c}^{2}$, scaled by a factor of 100 .

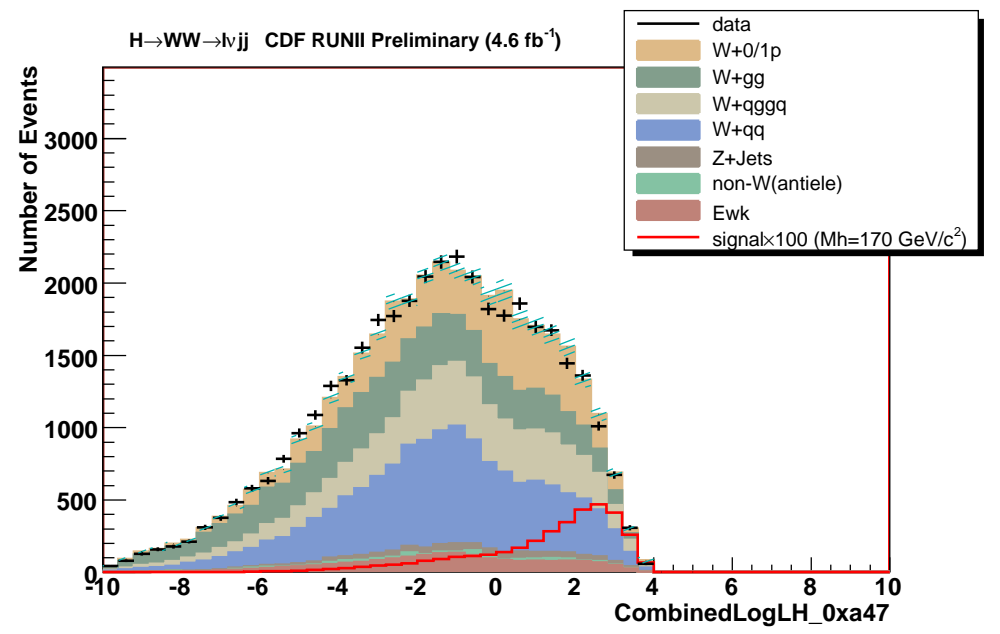

Figure B.6: Likelihood output for the analysis at $m_{H}=170 \mathrm{GeV} / \mathrm{c}^{2}$. The open red histogram shows the distribution for signal with $m_{H}=170 \mathrm{GeV} / \mathrm{c}^{2}$, scaled by a factor of 100 . 


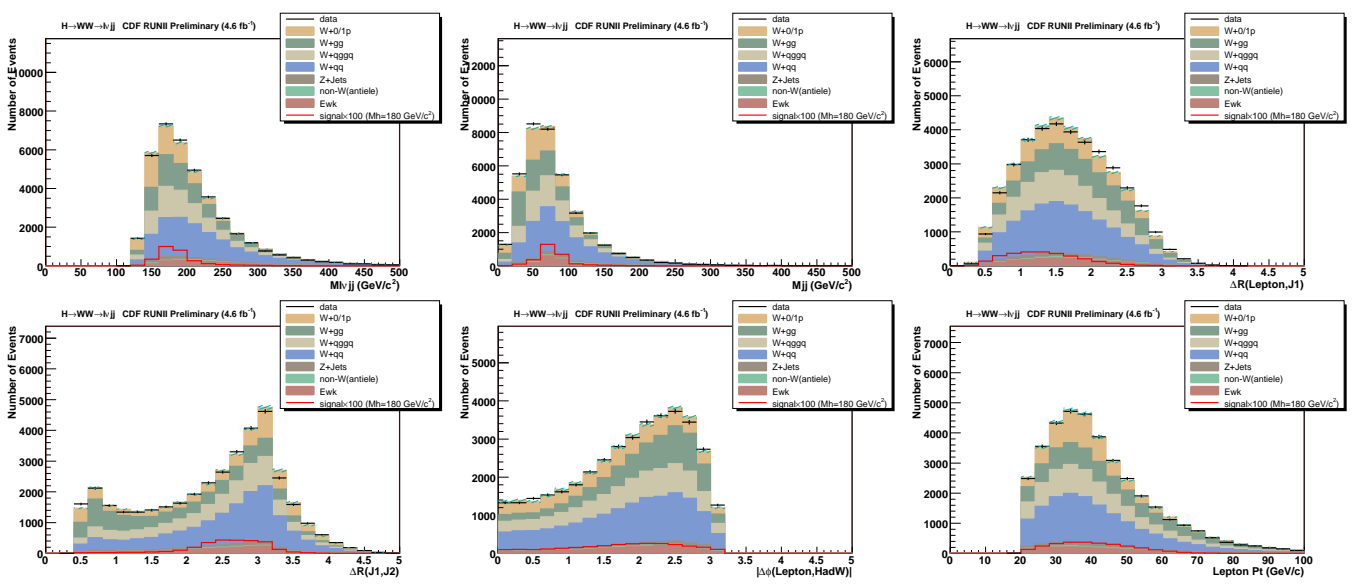

Figure B.7: Likelihood input distributions for $m_{H}=180 \mathrm{GeV} / \mathrm{c}^{2}$. The open red histogram shows the distribution for signal with $m_{H}=180 \mathrm{GeV} / \mathrm{c}^{2}$, scaled by a factor of 100 .

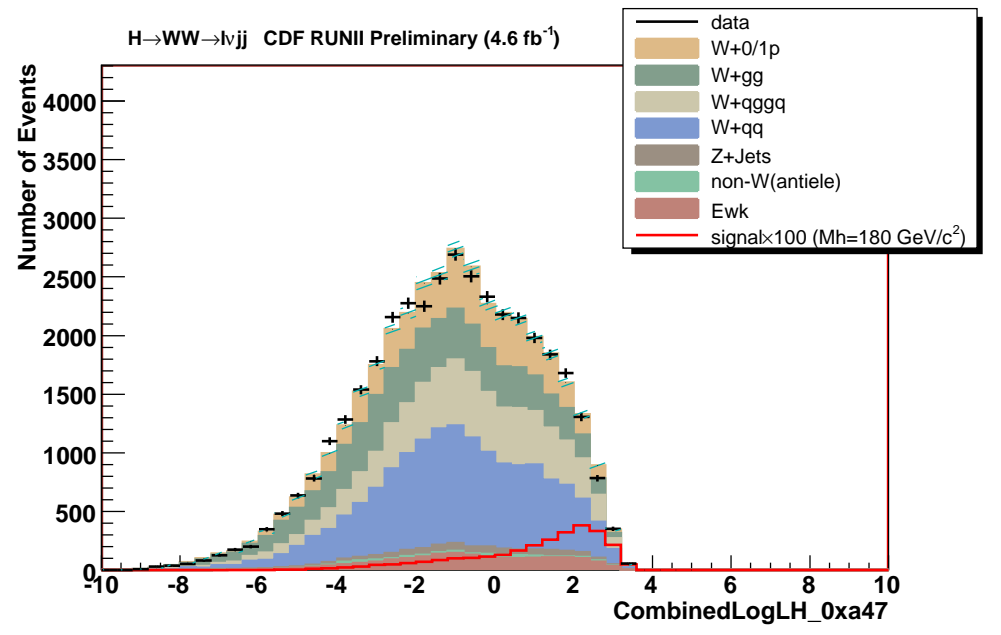

Figure B.8: Likelihood output distribution for $m_{H}=180 \mathrm{GeV} / \mathrm{c}^{2}$. The open red histogram shows the distribution for signal with $m_{H}=180 \mathrm{GeV} / \mathrm{c}^{2}$, scaled by a factor of 100 . 

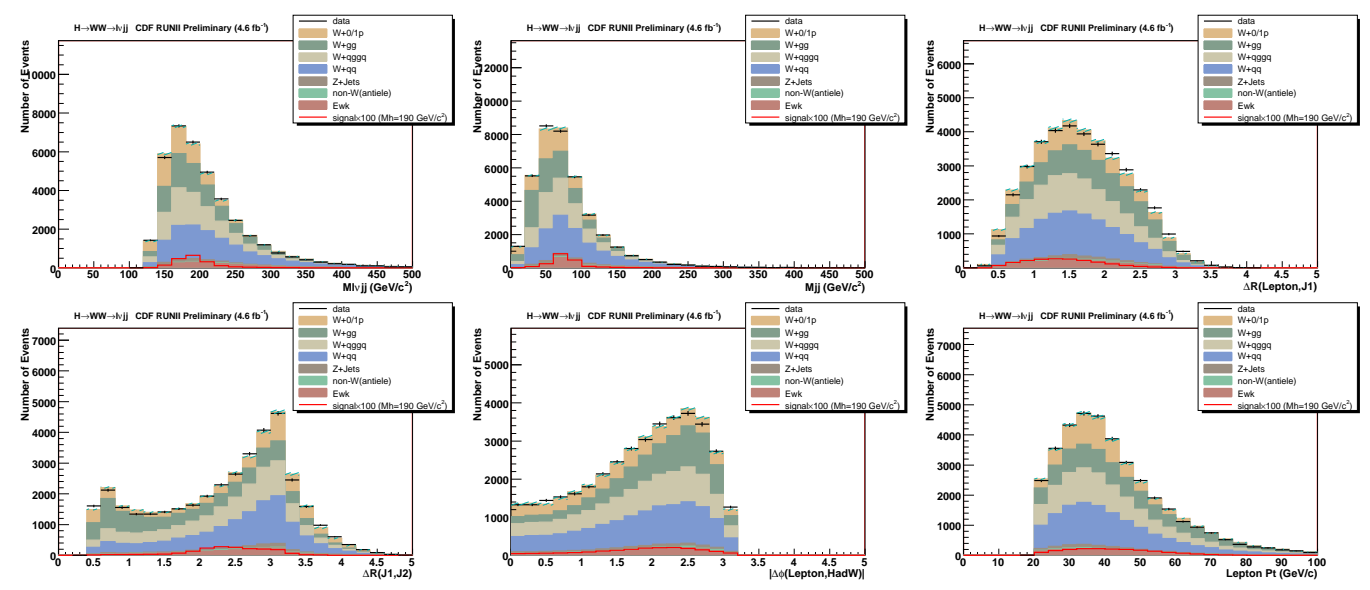

Figure B.9: Likelihood input distributions for $m_{H}=190 \mathrm{GeV} / \mathrm{c}^{2}$. The crosses shows the data, filled histograms are for backgrounds.

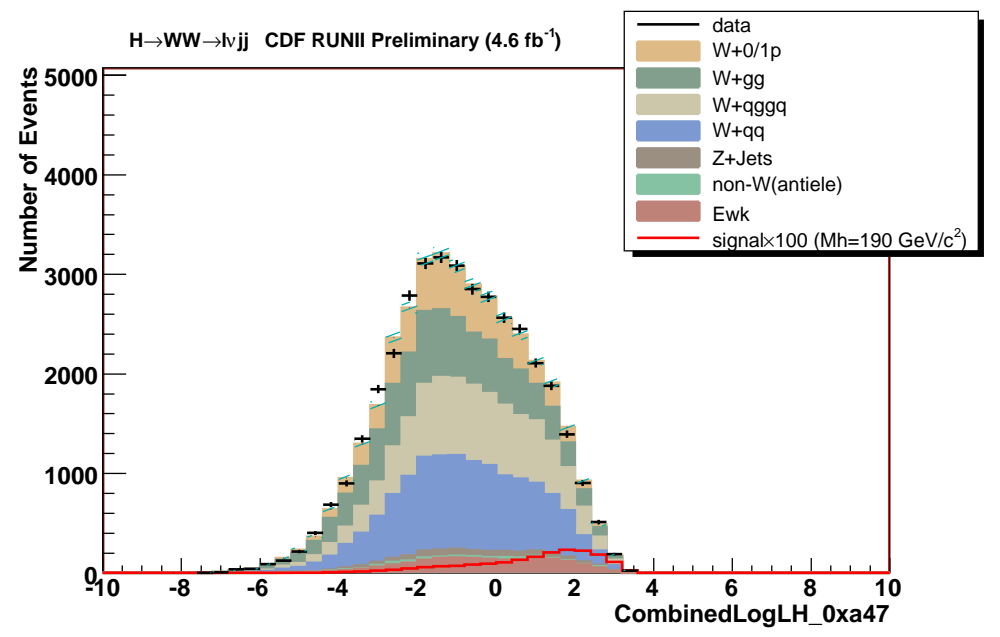

Figure B.10: Likelihood output distribution for $m_{H}=190 \mathrm{GeV} / \mathrm{c}^{2}$. The open red histogram shows the distribution for signal with $m_{H}=190 \mathrm{GeV} / \mathrm{c}^{2}$, scaled by a factor of 100 . 


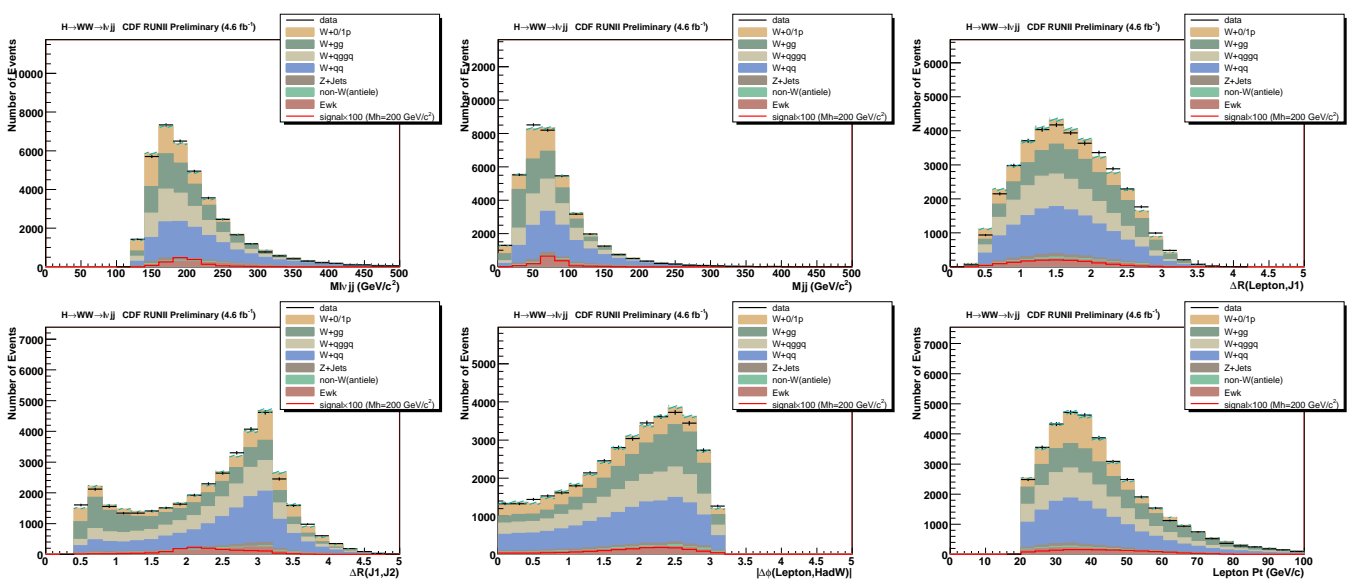

Figure B.11: Likelihood input distributions for $m_{H}=200 \mathrm{GeV} / \mathrm{c}^{2}$. The open red histogram shows the distribution for signal with $m_{H}=200 \mathrm{GeV} / \mathrm{c}^{2}$, scaled by a factor of 100 .

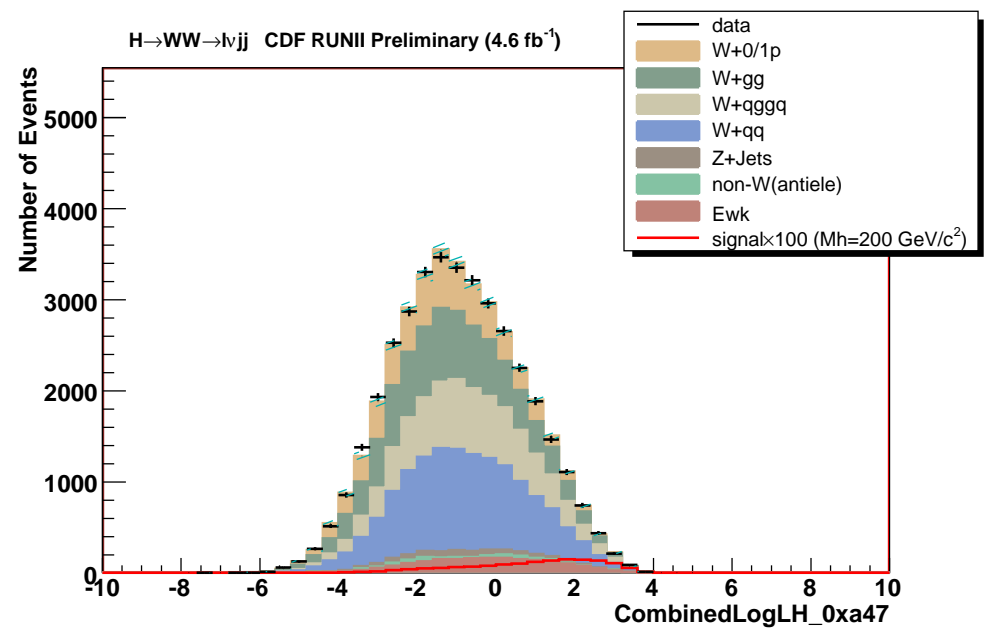

Figure B.12: Likelihood output distribution for $m_{H}=200 \mathrm{GeV} / \mathrm{c}^{2}$. The open red histogram shows the distribution for signal with $m_{H}=200 \mathrm{GeV} / \mathrm{c}^{2}$, scaled by a factor of 100 . 



\section{Appendix C}

\section{$\mathbf{Q}^{2}$ and JES Shape Systematics}

We estimate shape systematical uncertainties of the $W+$ jets using different $\mathrm{Q}^{2}$ Monte Carlo samples. Figure C. 1 - C.12 show distributions of the likelihood output for $W+$ jets samples. In these figures, the black, red and blue lines show the distribution of the center value, $+1 \sigma\left(2.0 \times \mathrm{Q}^{2}\right)$ and $-1 \sigma\left(0.5 \times \mathrm{Q}^{2}\right)$.
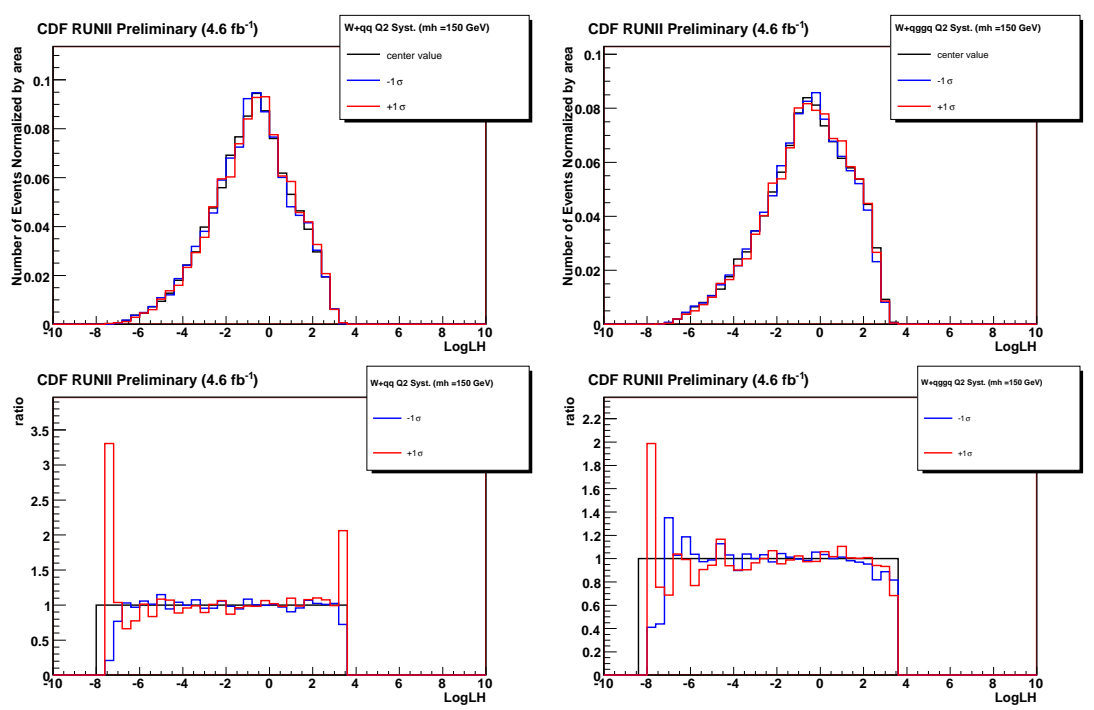

Figure C.1: Likelihood output distribution using the $W+$ jets $\mathrm{Q}^{2}$ systematic samples for $m_{H}=$ $150 \mathrm{GeV} / \mathrm{c}^{2}$. The left top plot shows log likelihood output for $W+\mathrm{qq}$ background. The left bottom plot shows ratios which are $\pm 1 \sigma /$ (center value). The right plots show log likelihood output for $W+$ qggq background. 

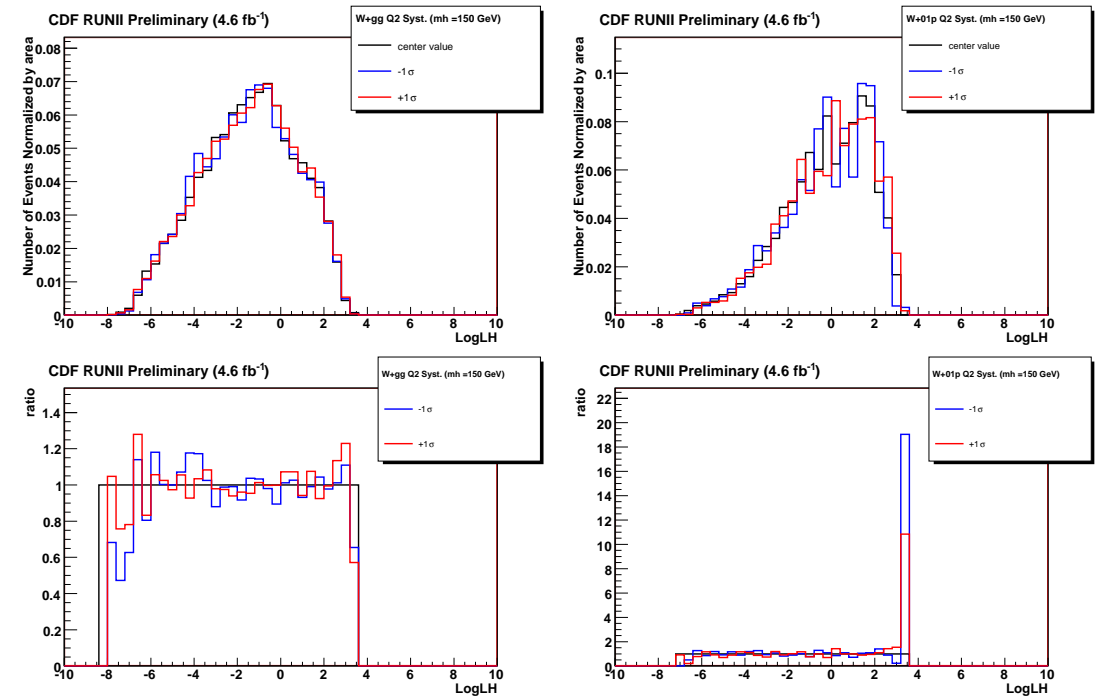

Figure C.2: Likelihood output distribution using the $W+$ jets $\mathrm{Q}^{2}$ systematic samples for $m_{H}=$ $150 \mathrm{GeV} / \mathrm{c}^{2}$. The left top plot shows log likelihood output for $W+\mathrm{gg}$ background. The left bottom plot shows ratios which are $\pm 1 \sigma /$ (center value). The right plots show log likelihood output for $W+0$ or 1 parton background. 

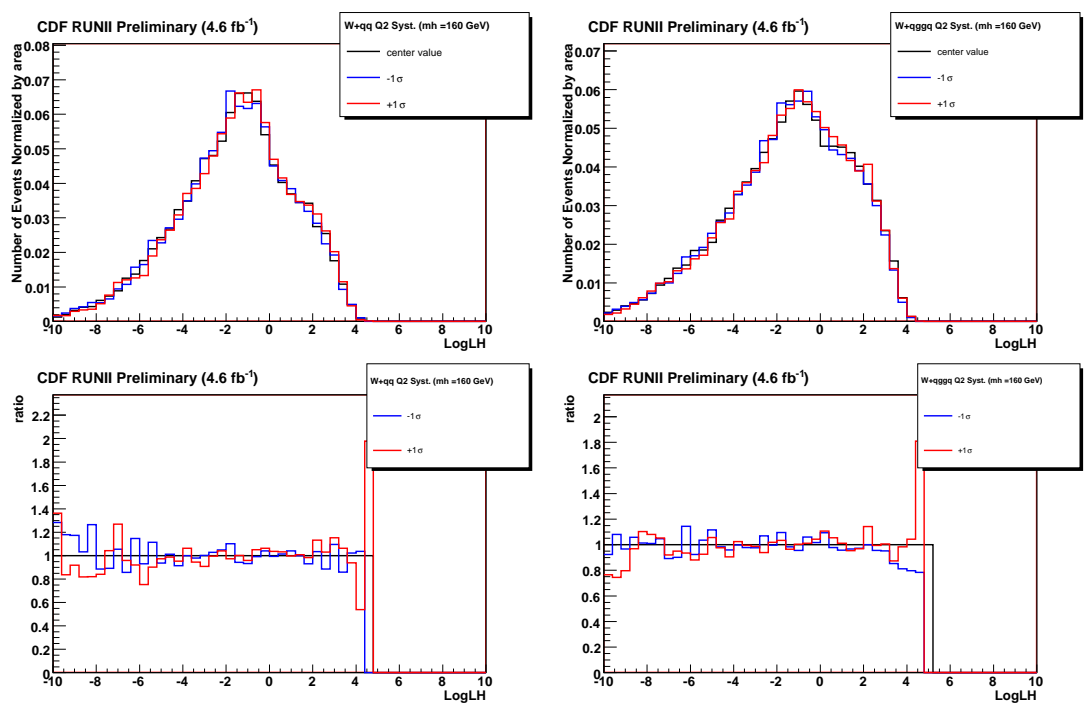

Figure C.3: Likelihood output distribution using the $W+$ jets $\mathrm{Q}^{2}$ systematic samples for $m_{H}=$ $160 \mathrm{GeV} / \mathrm{c}^{2}$. The left top plot shows log likelihood output for $W+\mathrm{qq}$ background. The left bottom plot shows ratios which are $\pm 1 \sigma /$ (center value). The right plots show log likelihood output for $W+$ qggq background. 

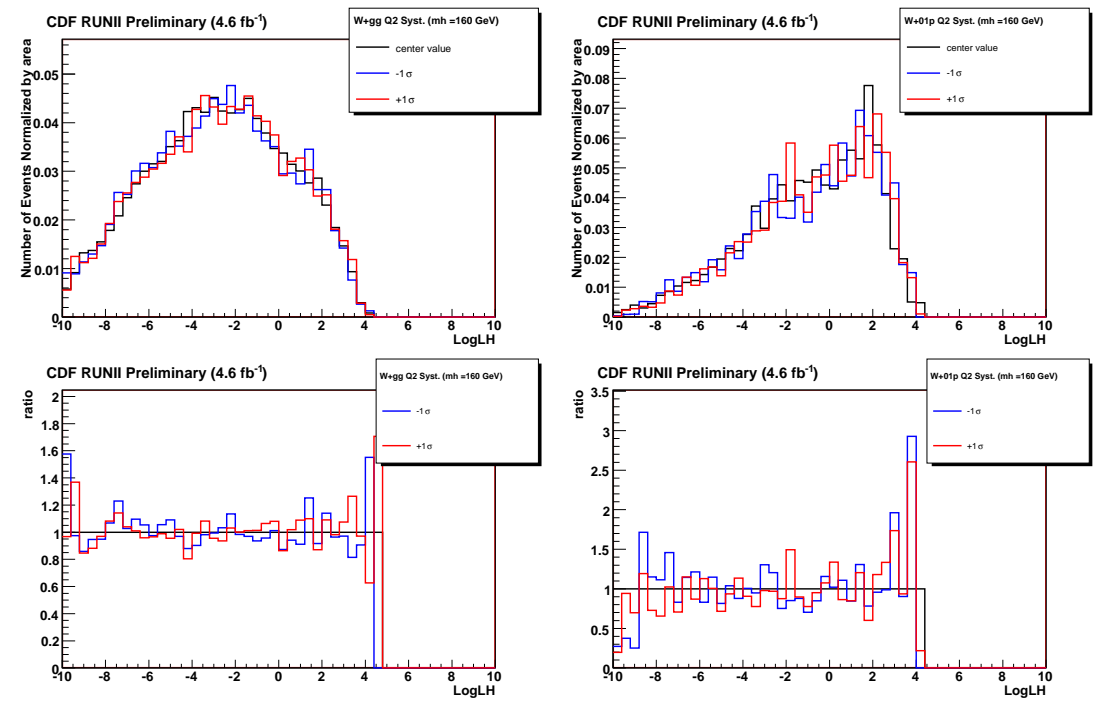

Figure C.4: Likelihood output distribution using the $W+$ jets $\mathrm{Q}^{2}$ systematic samples for $m_{H}=$ $160 \mathrm{GeV} / \mathrm{c}^{2}$. The left top plot shows log likelihood output for $W+\mathrm{gg}$ background. The left bottom plot shows ratios which are $\pm 1 \sigma /$ (center value). The right plots show log likelihood output for $W+0$ or 1 parton background. 

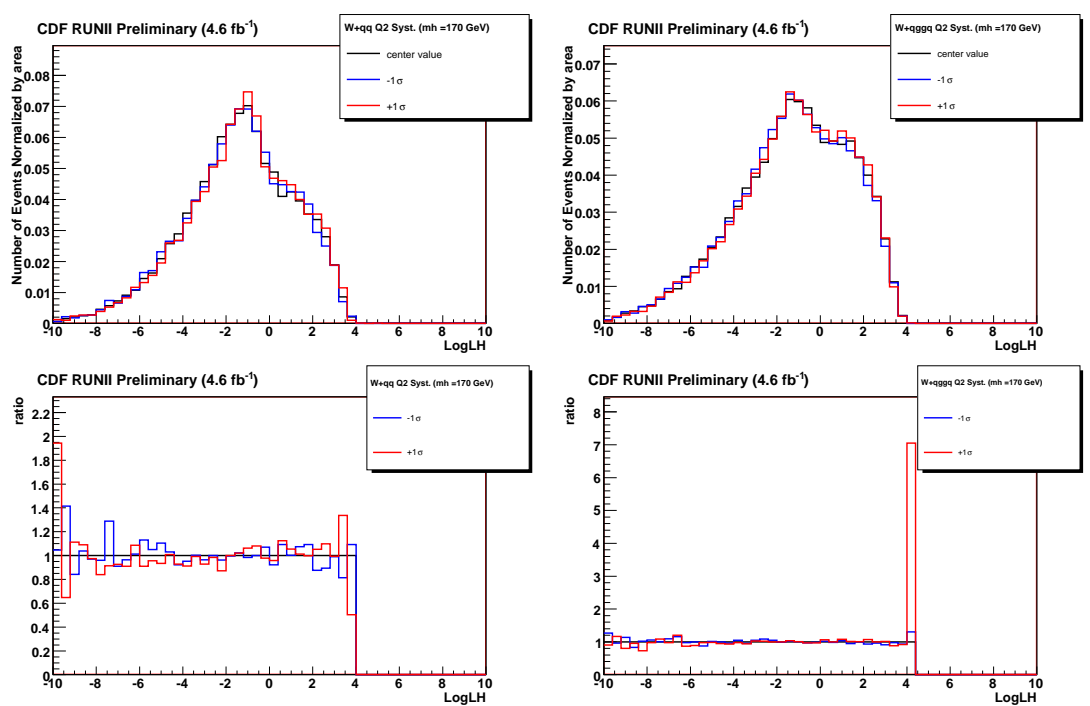

Figure C.5: Likelihood output distribution using the $W+$ jets $\mathrm{Q}^{2}$ systematic samples for $m_{H}=$ $170 \mathrm{GeV} / \mathrm{c}^{2}$. The left top plot shows log likelihood output for $W+\mathrm{qq}$ background. The left bottom plot shows ratios which are $\pm 1 \sigma /$ (center value). The right plots show log likelihood output for $W$ +qggq background. 

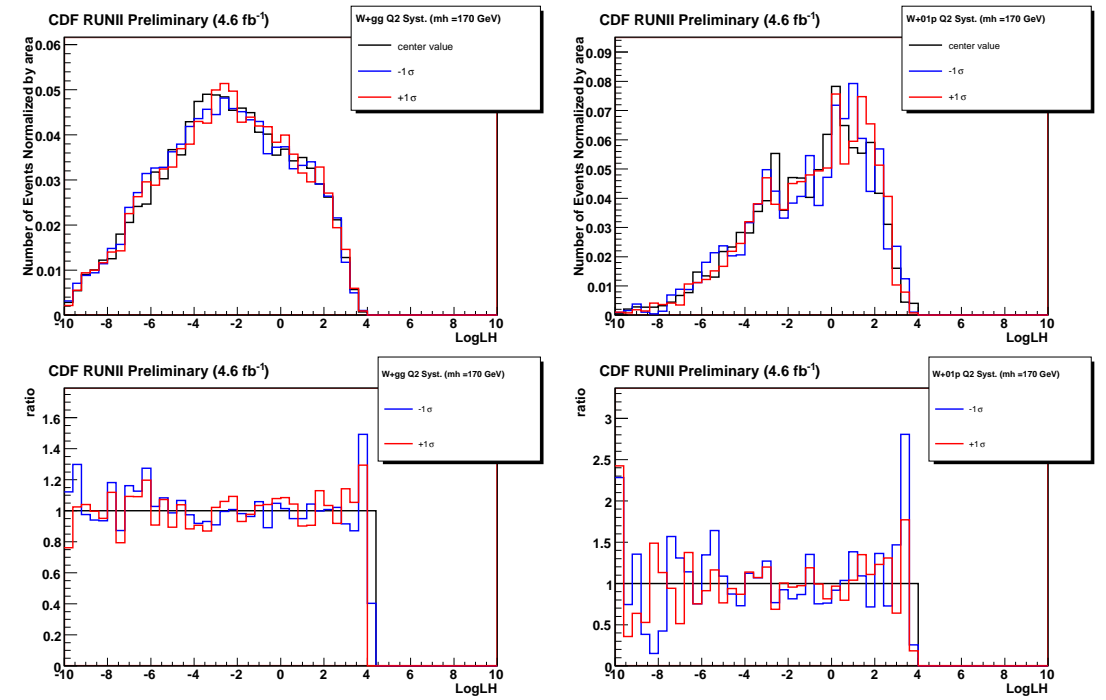

Figure C.6: Likelihood output distribution using the $W+$ jets $\mathrm{Q}^{2}$ systematic samples. The left top plot shows log likelihood output for $W+\mathrm{gg}$ background. The left bottom plot shows ratios which are $\pm 1 \sigma /$ (center value). The right plots show log likelihood output for $W+0$ or 1 parton background. 

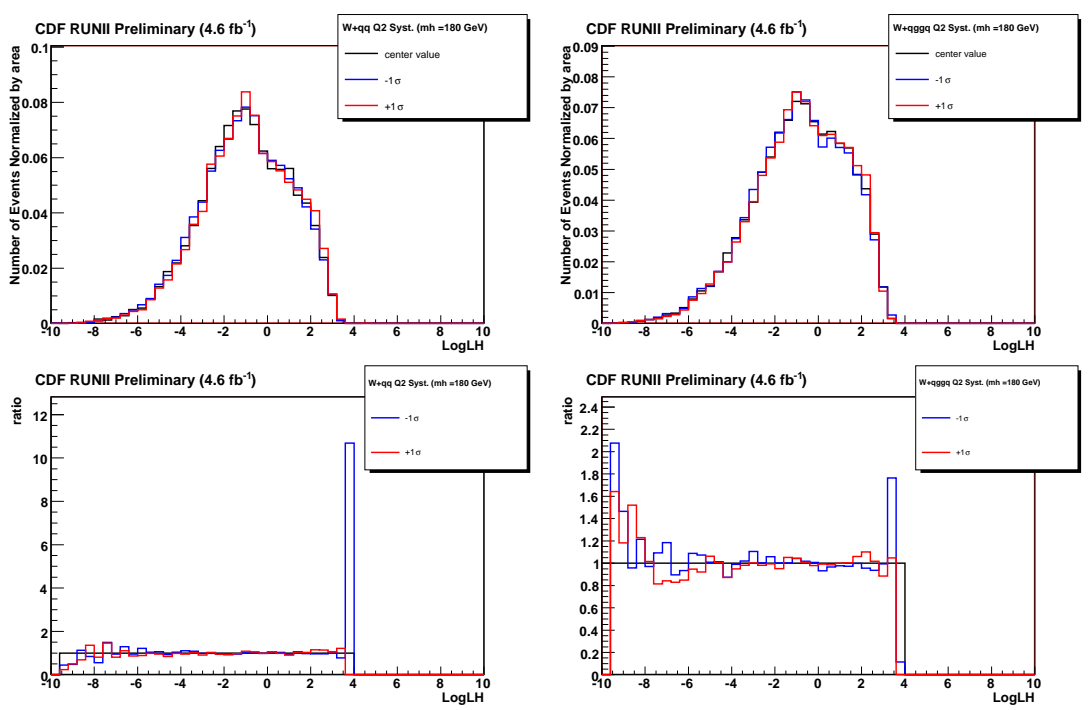

Figure C.7: Likelihood output distribution using the $W+$ jets $\mathrm{Q}^{2}$ systematic samples for $m_{H}=$ $180 \mathrm{GeV} / \mathrm{c}^{2}$. The left top plot shows log likelihood output for $W+\mathrm{qq}$ background. The left bottom plot shows ratios which are $\pm 1 \sigma /$ (center value). The right plots show log likelihood output for $W+$ qggq background. 

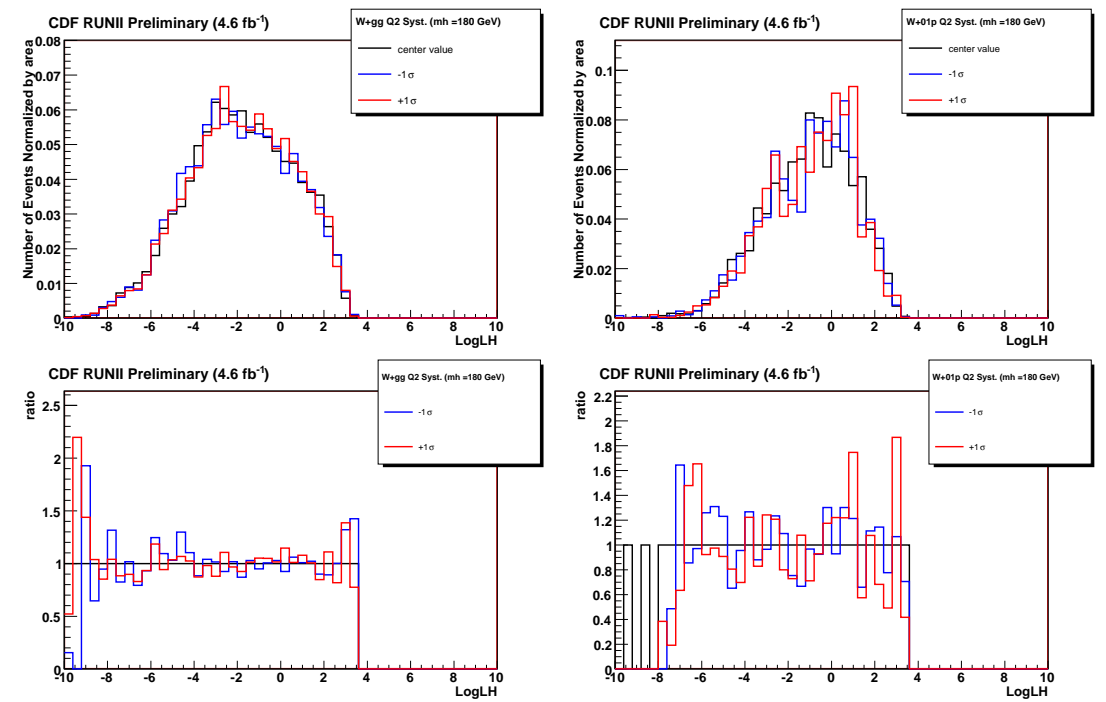

Figure C.8: Likelihood output distribution using the $W+$ jets $\mathrm{Q}^{2}$ systematic samples. The left top plot shows log likelihood output for $W+\mathrm{gg}$ background. The left bottom plot shows ratios which are $\pm 1 \sigma /$ (center value). The right plots show log likelihood output for $W+0$ or 1 parton background. 

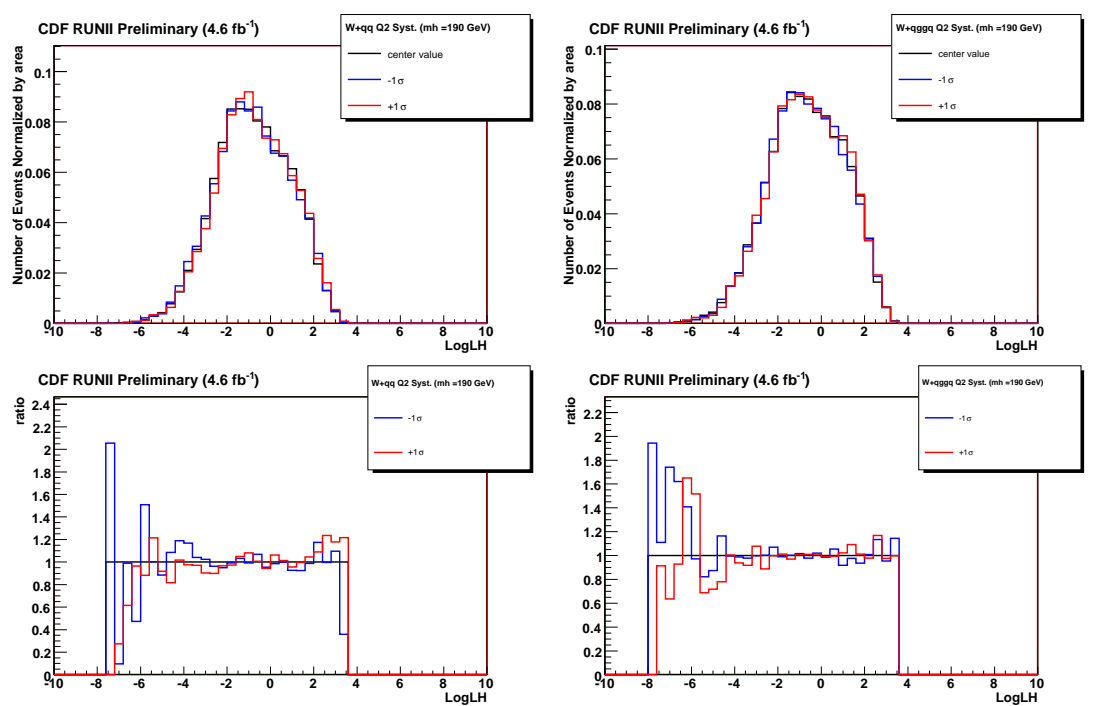

Figure C.9: Likelihood output distribution using the $W+$ jets $\mathrm{Q}^{2}$ systematic samples for $m_{H}=$ $190 \mathrm{GeV} / \mathrm{c}^{2}$. The left top plot shows log likelihood output for $W+\mathrm{qq}$ background. The left bottom plot shows ratios which are $\pm 1 \sigma /$ (center value). The right plots show log likelihood output for $W+$ qggq background. 

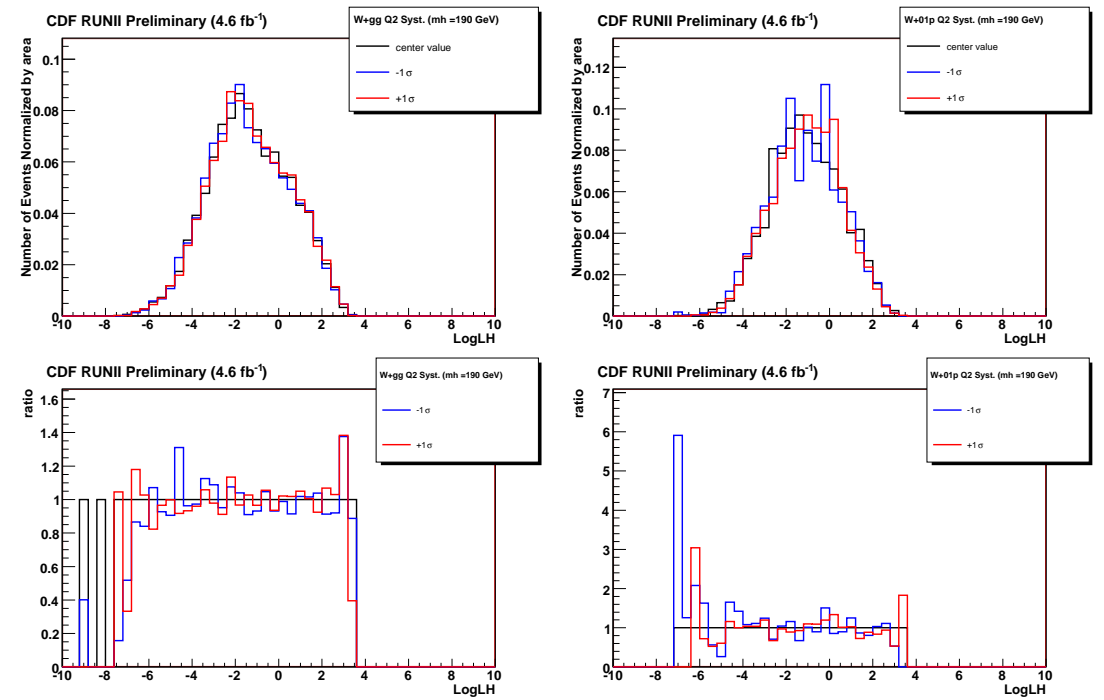

Figure C.10: Likelihood output distribution using the $W+$ jets $\mathrm{Q}^{2}$ systematic samples. The left top plot shows log likelihood output for $W+\mathrm{gg}$ background. The left bottom plot shows ratios which are $\pm 1 \sigma /$ (center value). The right plots show log likelihood output for $W+0$ or 1 parton background. 

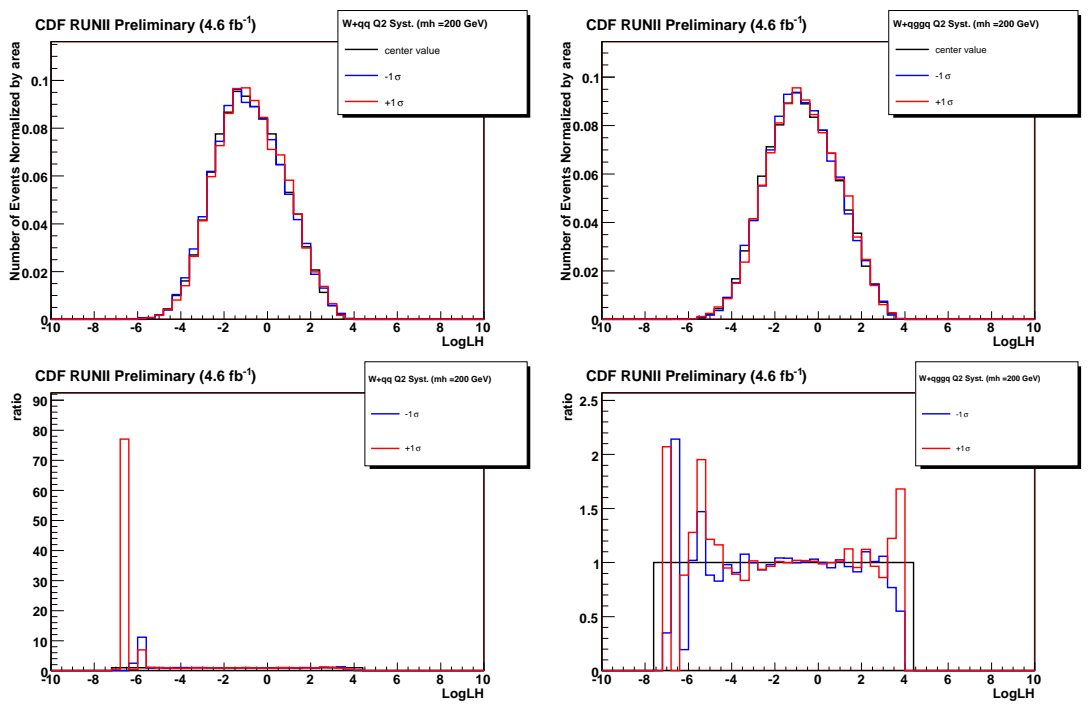

Figure C.11: Likelihood output distribution using the $W+$ jets $\mathrm{Q}^{2}$ systematic samples for $m_{H}=$ $200 \mathrm{GeV} / \mathrm{c}^{2}$. The left top plot shows log likelihood output for $W+\mathrm{qq}$ background. The left bottom plot shows ratios which are $\pm 1 \sigma /$ (center value). The right plots show log likelihood output for $W+$ qggq background. 

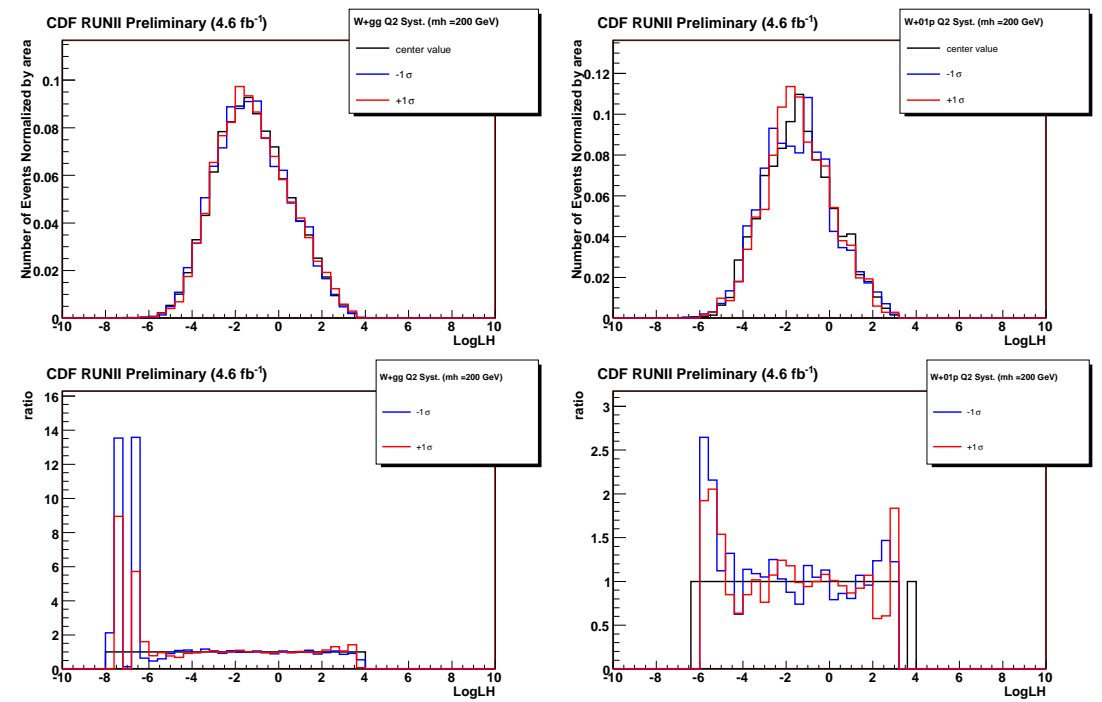

Figure C.12: Likelihood output distribution using the $W+$ jets $\mathrm{Q}^{2}$ systematic samples. The left top plot shows log likelihood output for $W+\mathrm{gg}$ background. The left bottom plot shows ratios which are $\pm 1 \sigma /$ (center value). The right plots show log likelihood output for $W+0$ or 1 parton background. 
We also estimate shape systematical uncertainty related to JES for $W+$ jets background. We have 4 kind fo JES systematics which are $(q+1 \sigma, g \pm 0 \sigma),(q-1 \sigma, g \pm 0 \sigma),(q \pm 0 \sigma, g+1 \sigma)$ and $(q \pm 0 \sigma, g-1 \sigma)$. Figure C.13-C.36 show distributions of the likelihood output for $W+$ jets samples. In these figures, the black, red and blue lines show the distribution of the center value, $+1 \sigma$ and $-1 \sigma$.
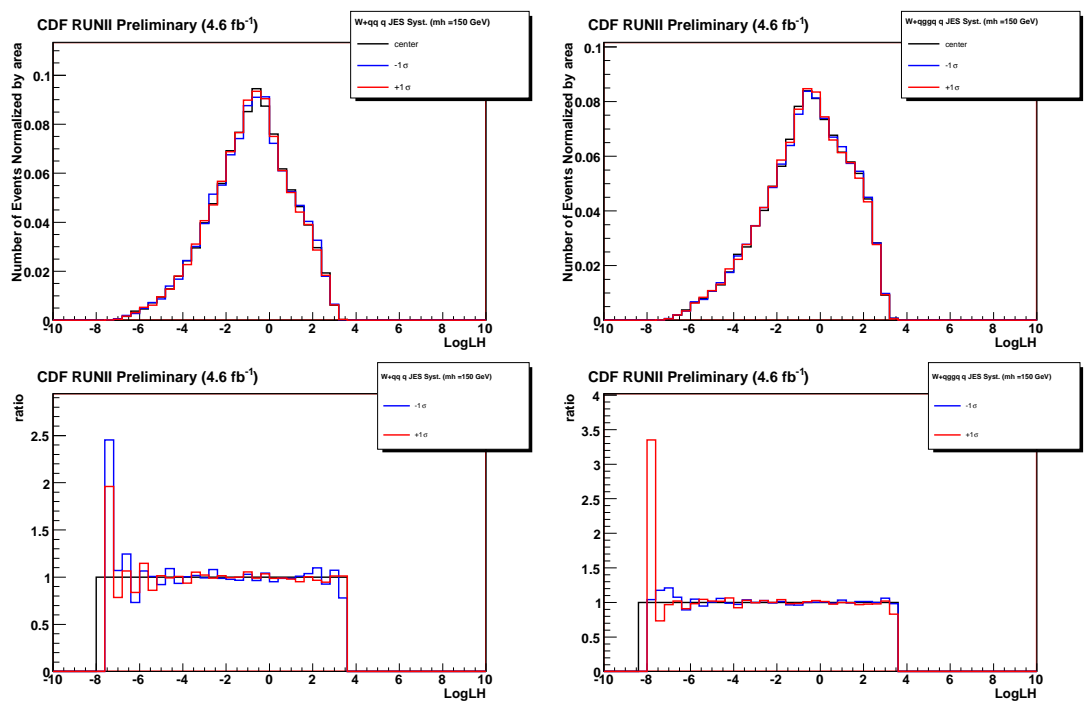

Figure C.13: Likelihood output distribution using the $W+$ jets quark JES $+1 \sigma$ systematic samples for $m_{H}=150 \mathrm{GeV} / \mathrm{c}^{2}$. The left top plot shows $\log$ likelihood output for $W+\mathrm{qq}$ background. The left bottom plot shows ratios which are $\pm 1 \sigma$ /(center value). The right plots show log likelihood output for $W+$ qggq background. 

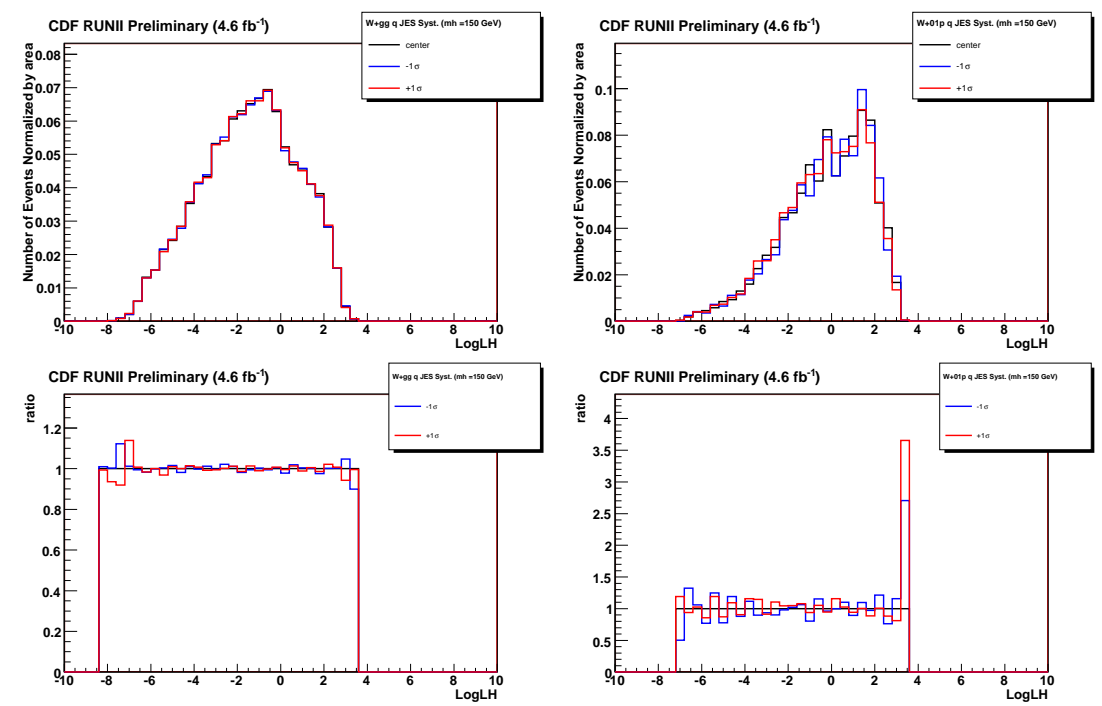

Figure C.14: Likelihood output distribution using the $W+$ jets quark JES $\pm 1 \sigma$ systematic samples for $m_{H}=150 \mathrm{GeV} / \mathrm{c}^{2}$. The left top plot shows log likelihood output for $W+\mathrm{gg}$ background. The left bottom plot shows ratios which are $\pm 1 \sigma$ /(center value). The right plots show log likelihood output for $W+0$ or 1 parton background.
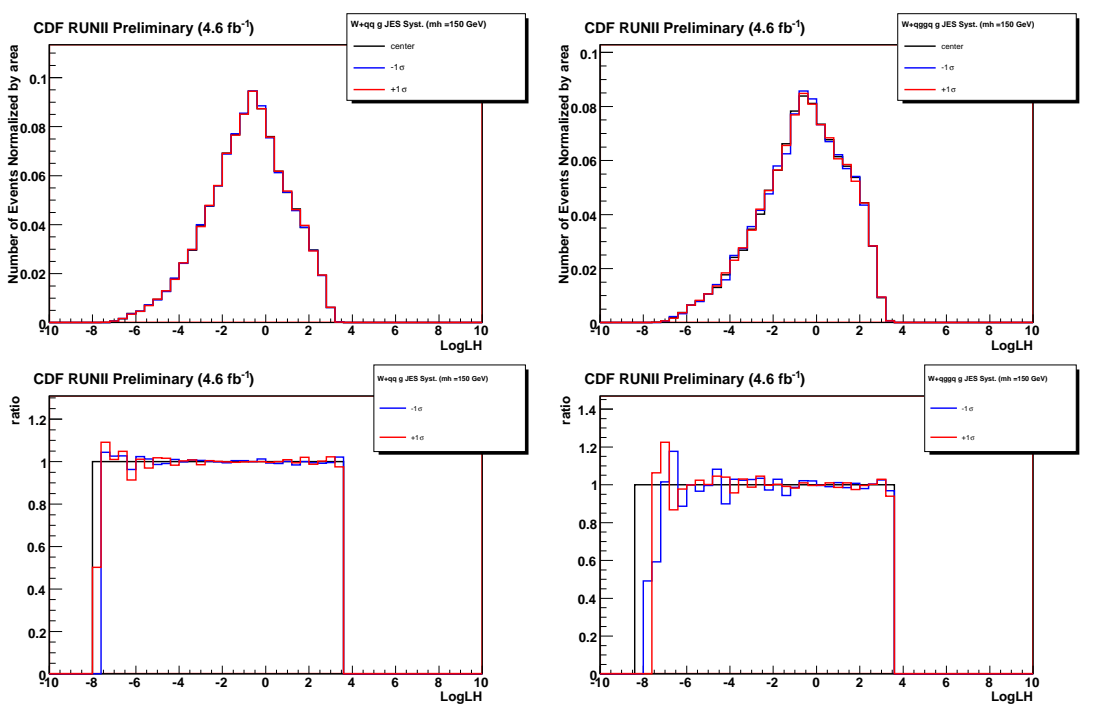

Figure C.15: Likelihood output distribution using the $W+$ jets gluon JES $+1 \sigma$ systematic samples for $m_{H}=150 \mathrm{GeV} / \mathrm{c}^{2}$. The left top plot shows log likelihood output for $W+\mathrm{qq}$ background. The left bottom plot shows ratios which are $\pm 1 \sigma$ /(center value). The right plots show log likelihood output for $W+$ qggq background. 

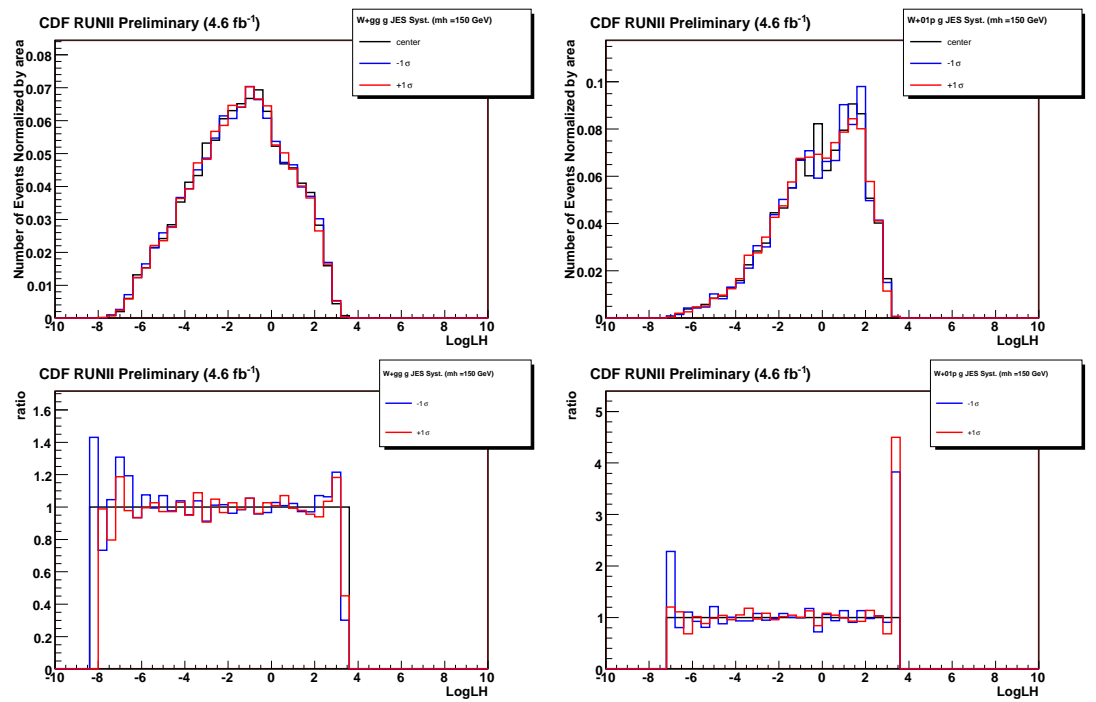

Figure C.16: Likelihood output distribution using the $W+$ jets gluon JES $\pm 1 \sigma$ systematic samples for $m_{H}=150 \mathrm{GeV} / \mathrm{c}^{2}$. The left top plot shows $\log$ likelihood output for $W+\mathrm{gg}$ background. The left bottom plot shows ratios which are $\pm 1 \sigma /$ (center value). The right plot shows log likelihood output for $W+0$ or 1 parton background. 

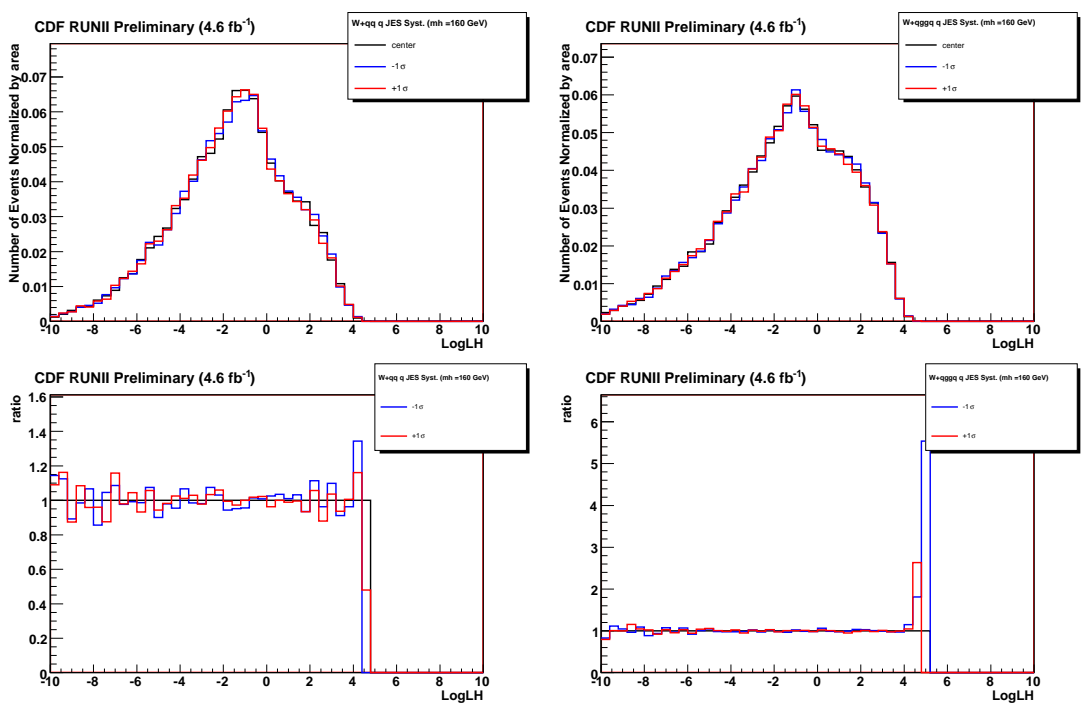

Figure C.17: Likelihood output distribution using the $W+$ jets quark JES $+1 \sigma$ systematic samples for $m_{H}=160 \mathrm{GeV} / \mathrm{c}^{2}$. The left top plot shows log likelihood output for $W+q q$ background. The left bottom plot shows ratios which are $\pm 1 \sigma /$ (center value). The right plots show log likelihood output for $W+$ qggq background. 

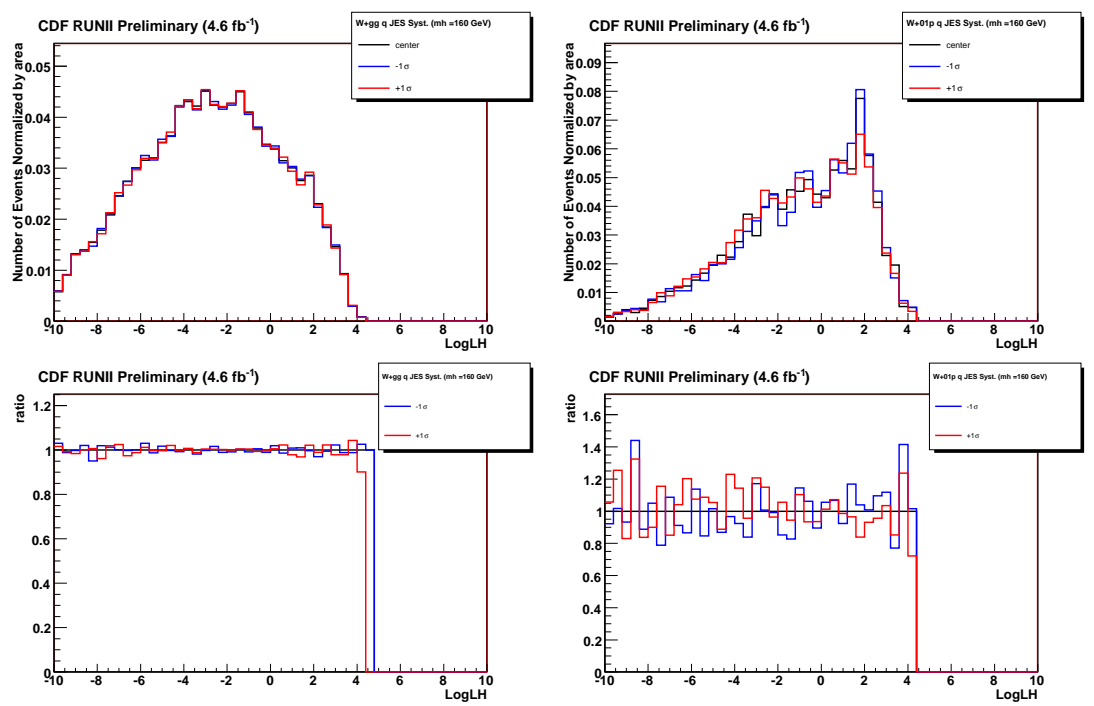

Figure C.18: Likelihood output distribution using the $W+$ jets quark JES $\pm 1 \sigma$ systematic samples for $m_{H}=160 \mathrm{GeV} / \mathrm{c}^{2}$. The left top plot shows log likelihood output for $W+$ gg background. The left bottom plot shows ratios which are $\pm 1 \sigma /$ (center value). The right plots show log likelihood output for $W+0$ or 1 parton background.
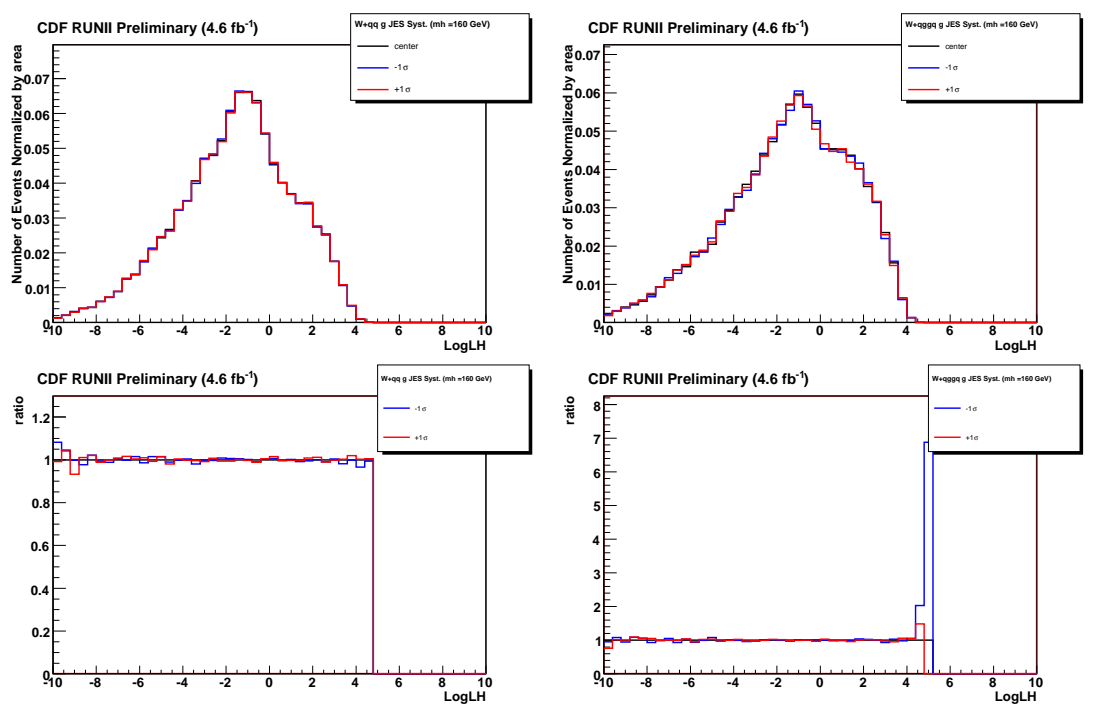

Figure C.19: Likelihood output distribution using the $W+$ jets gluon JES $+1 \sigma$ systematic samples for $m_{H}=160 \mathrm{GeV} / \mathrm{c}^{2}$. The left top plot shows $\log$ likelihood output for $W+\mathrm{qq}$ background. The left bottom plot shows ratios which are $\pm 1 \sigma /($ center value). The right plots show log likelihood output for $W+$ qggq background. 

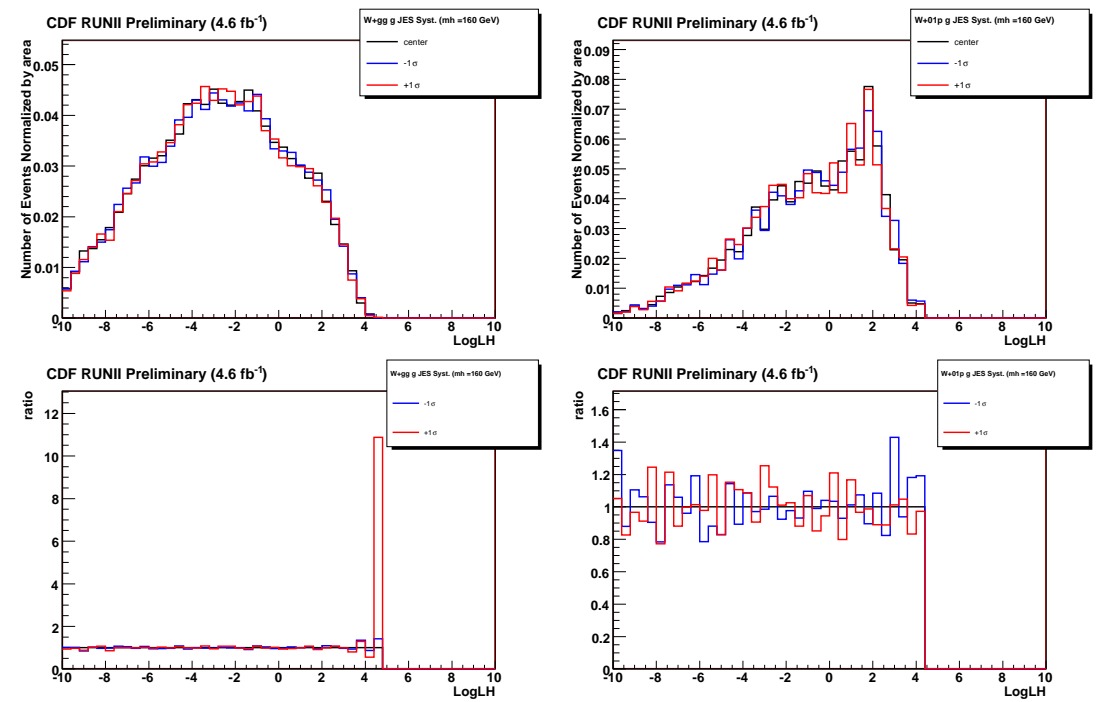

Figure C.20: Likelihood output distribution using the $W+$ jets gluon JES $\pm 1 \sigma$ systematic samples for $m_{H}=160 \mathrm{GeV} / \mathrm{c}^{2}$. The left top plot shows log likelihood output for $W+\mathrm{gg}$ background. The left bottom plot shows ratios which are $\pm 1 \sigma$ /(center value). The right plot shows log likelihood output for $W+0$ or 1 parton background. 

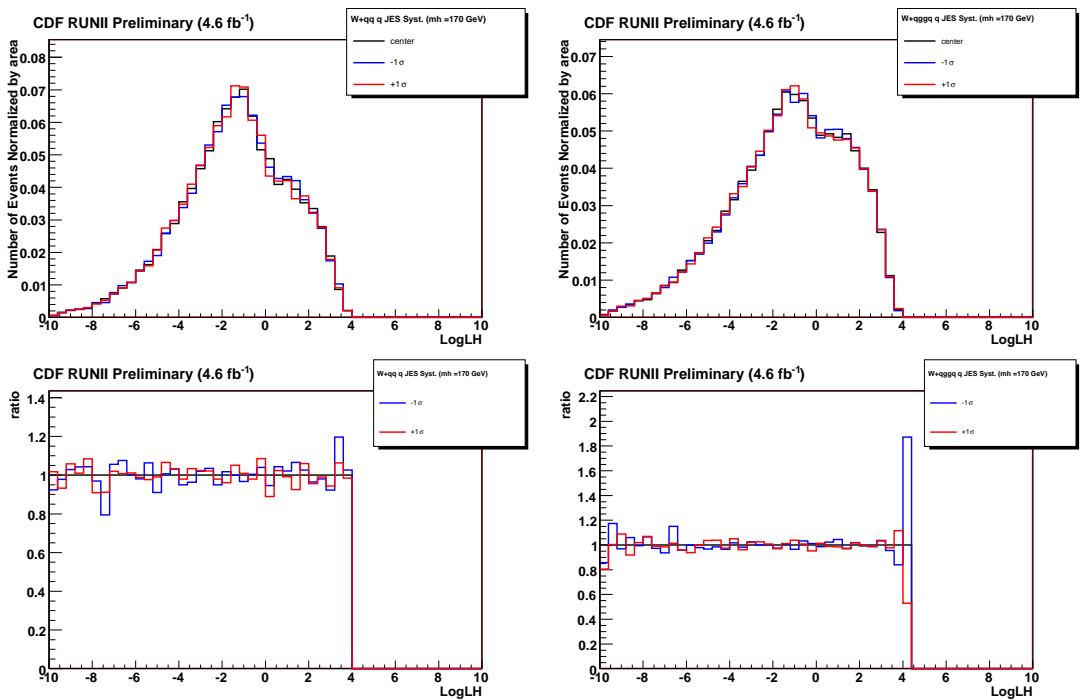

Figure C.21: Likelihood output distribution using the $W+$ jets quark JES $+1 \sigma$ systematic samples for $m_{H}=170 \mathrm{GeV} / \mathrm{c}^{2}$. The left top plot shows $\log$ likelihood output for $W+\mathrm{qq}$ background. The left bottom plot shows ratios which are $\pm 1 \sigma /$ (center value). The right plots show log likelihood output for $W+$ qggq background. 

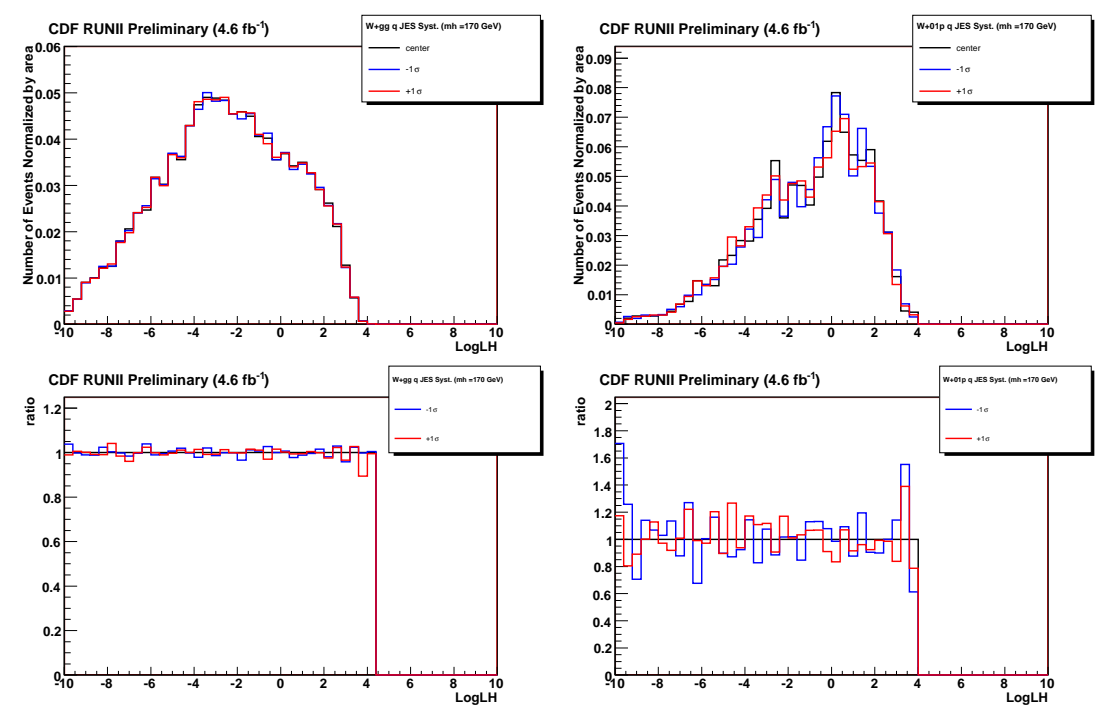

Figure C.22: Likelihood output distribution using the $W+$ jets quark JES $\pm 1 \sigma$ systematic samples for $m_{H}=170 \mathrm{GeV} / \mathrm{c}^{2}$. The left top plot shows log likelihood output for $W+\mathrm{gg}$ background. The left bottom plot shows ratios which are $\pm 1 \sigma$ /(center value). The right plots show log likelihood output for $W+0$ or 1 parton background.
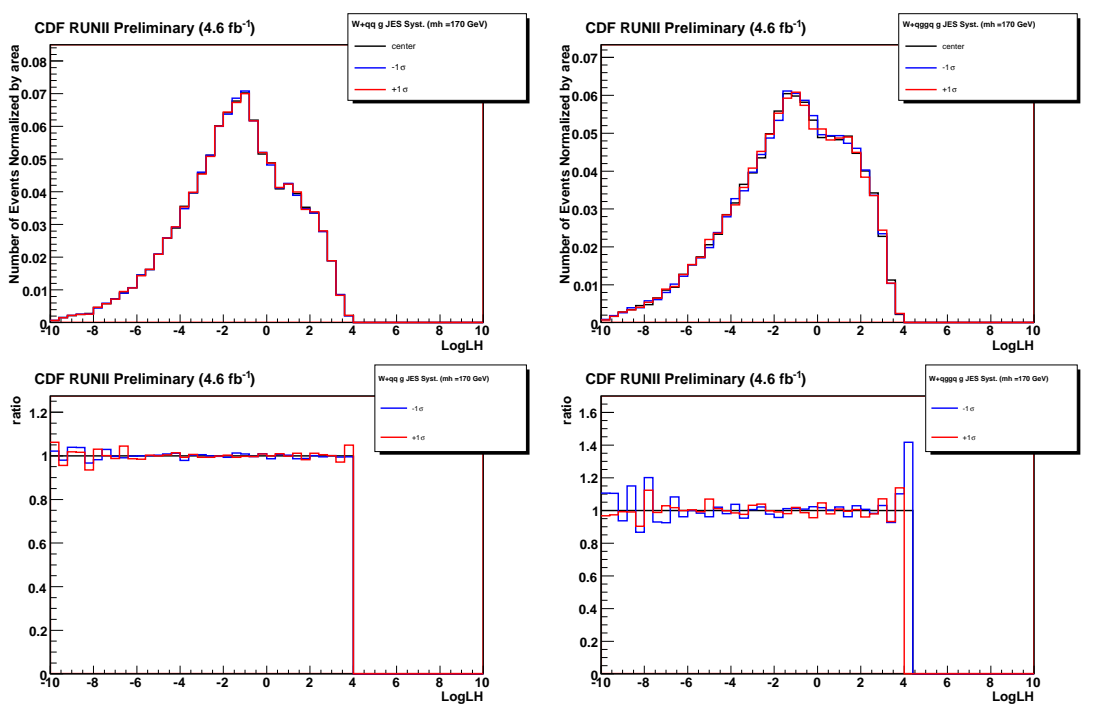

Figure C.23: Likelihood output distribution using the $W+$ jets gluon JES $+1 \sigma$ systematic samples for $m_{H}=170 \mathrm{GeV} / \mathrm{c}^{2}$. The left top plot shows log likelihood output for $W+\mathrm{qq}$ background. The left bottom plot shows ratios which are $\pm 1 \sigma$ /(center value). The right plots show log likelihood output for $W+$ qggq background. 

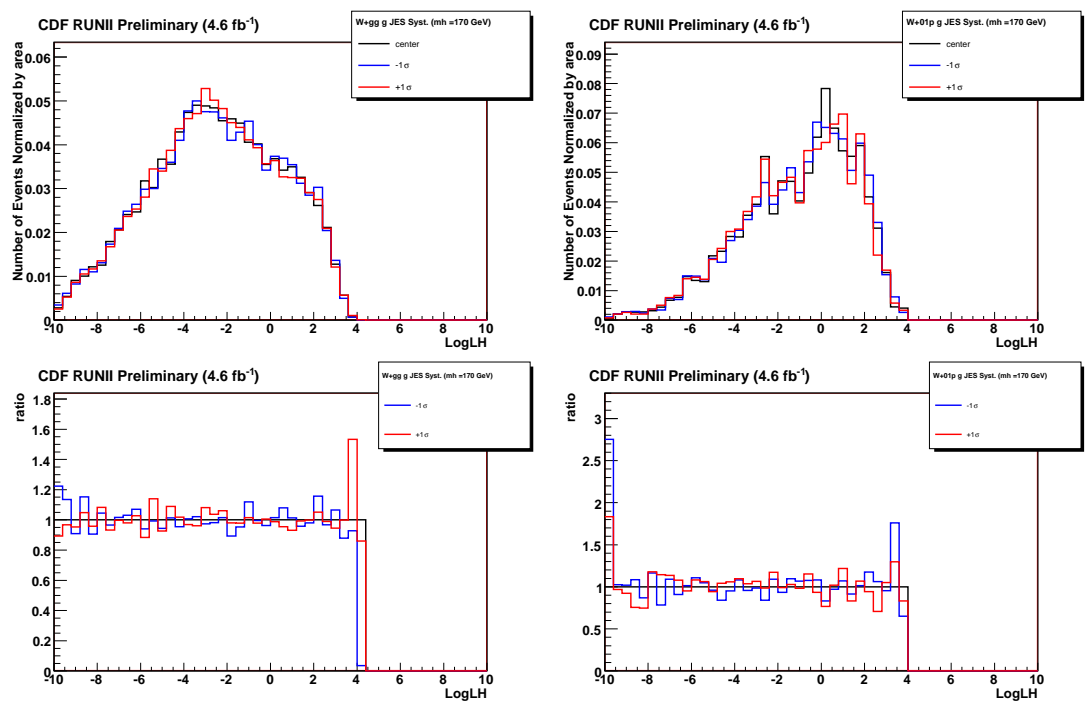

Figure C.24: Likelihood output distribution using the $W+$ jets gluon JES $\pm 1 \sigma$ systematic samples for $m_{H}=170 \mathrm{GeV} / \mathrm{c}^{2}$. The left top plot shows $\log$ likelihood output for $W+\mathrm{gg}$ background. The left bottom plot shows ratios which are $\pm 1 \sigma /$ (center value). The right plot shows log likelihood output for $W+0$ or 1 parton background. 

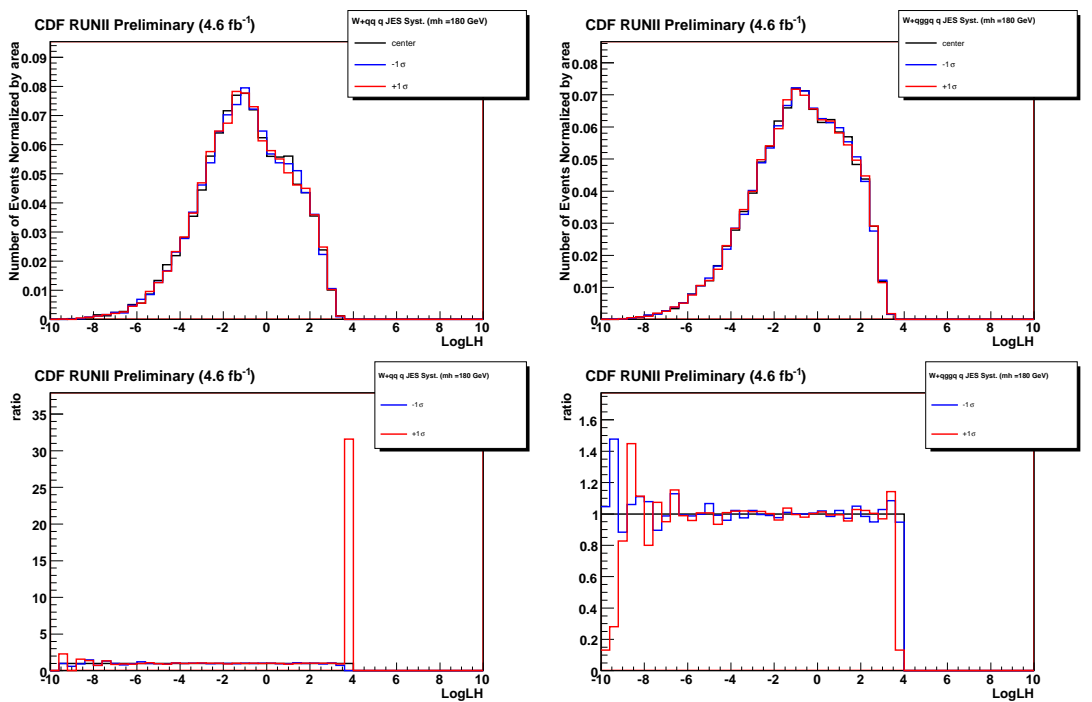

Figure C.25: Likelihood output distribution using the $W+$ jets quark JES $+1 \sigma$ systematic samples for $m_{H}=180 \mathrm{GeV} / \mathrm{c}^{2}$. The left top plot shows log likelihood output for $W+q q$ background. The left bottom plot shows ratios which are $\pm 1 \sigma /$ (center value). The right plots show log likelihood output for $W+q g g q$ background. 

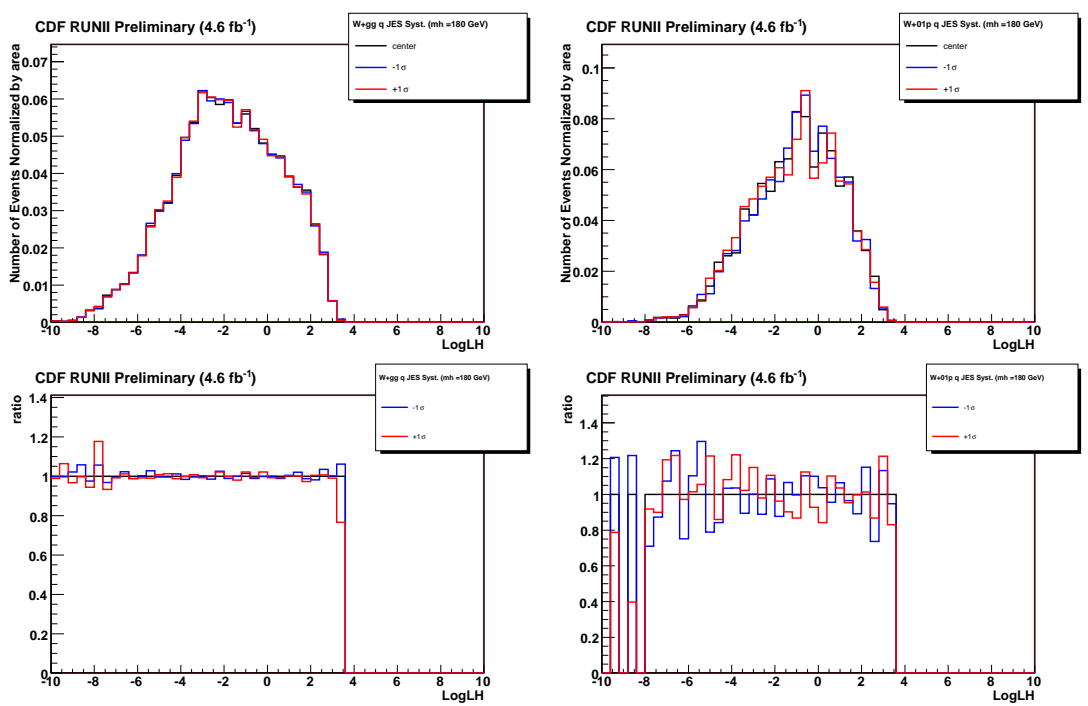

Figure C.26: Likelihood output distribution using the $W+$ jets quark JES $\pm 1 \sigma$ systematic samples for $m_{H}=180 \mathrm{GeV} / \mathrm{c}^{2}$. The left top plot shows log likelihood output for $W+$ gg background. The left bottom plot shows ratios which are $\pm 1 \sigma /$ (center value). The right plots show log likelihood output for $W+0$ or 1 parton background.
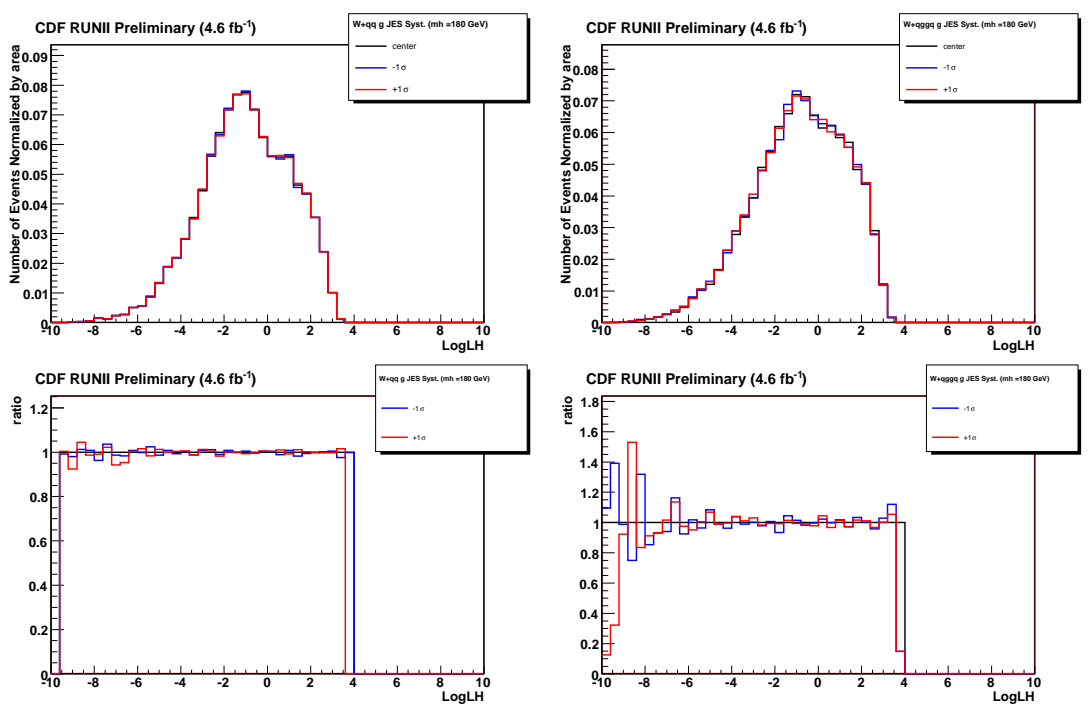

Figure C.27: Likelihood output distribution using the $W+$ jets gluon JES $+1 \sigma$ systematic samples for $m_{H}=180 \mathrm{GeV} / \mathrm{c}^{2}$. The left top plot shows $\log$ likelihood output for $W+\mathrm{qq}$ background. The left bottom plot shows ratios which are $\pm 1 \sigma /($ center value). The right plots show log likelihood output for $W+$ qggq background. 

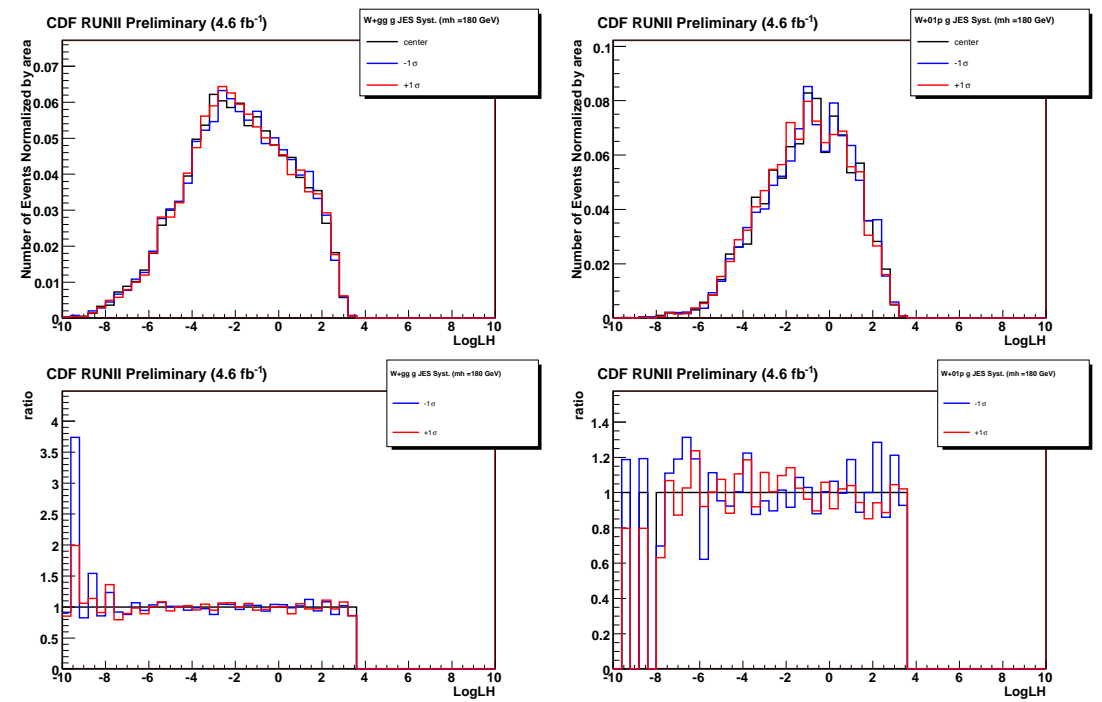

Figure C.28: Likelihood output distribution using the $W+$ jets gluon JES $\pm 1 \sigma$ systematic samples for $m_{H}=180 \mathrm{GeV} / \mathrm{c}^{2}$. The left top plot shows log likelihood output for $W+\mathrm{gg}$ background. The left bottom plot shows ratios which are $\pm 1 \sigma$ /(center value). The right plot shows log likelihood output for $W+0$ or 1 parton background. 

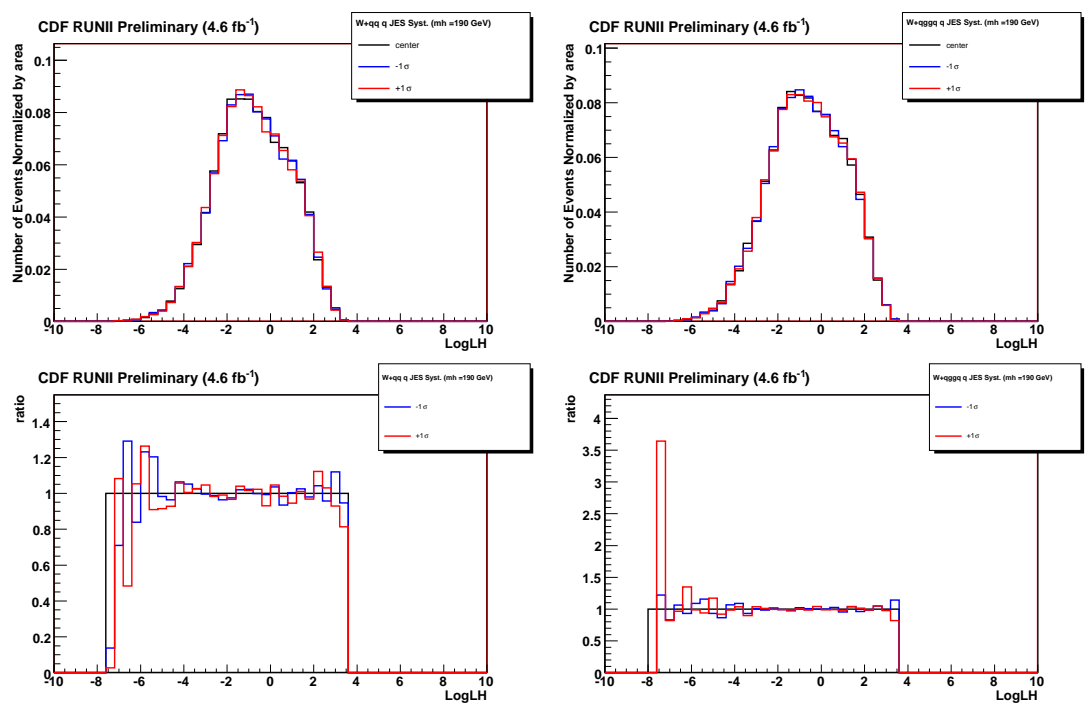

Figure C.29: Likelihood output distribution using the $W+$ jets quark JES $+1 \sigma$ systematic samples for $m_{H}=190 \mathrm{GeV} / \mathrm{c}^{2}$. The left top plot shows log likelihood output for $W+\mathrm{qq}$ background. The left bottom plot shows ratios which are $\pm 1 \sigma /$ (center value). The right plots show log likelihood output for $W+$ qggq background. 

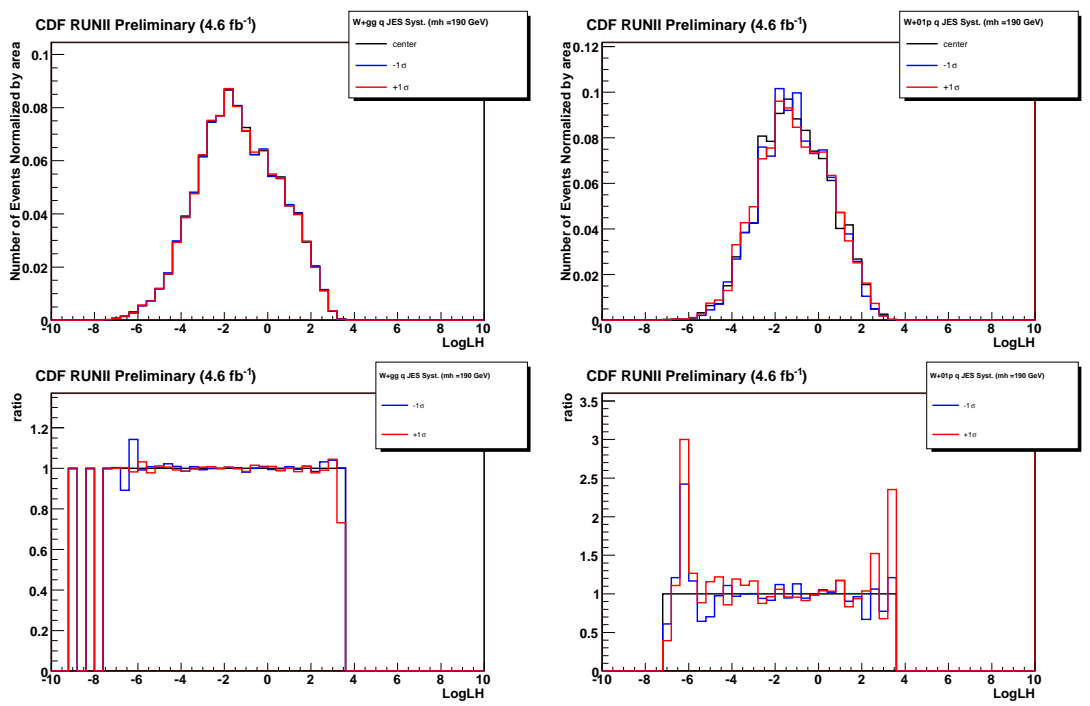

Figure C.30: Likelihood output distribution using the $W+$ jets quark JES $\pm 1 \sigma$ systematic samples for $m_{H}=190 \mathrm{GeV} / \mathrm{c}^{2}$. The left top plot shows log likelihood output for $W+\mathrm{gg}$ background. The left bottom plot shows ratios which are $\pm 1 \sigma$ /(center value). The right plots show log likelihood output for $W+0$ or 1 parton background.
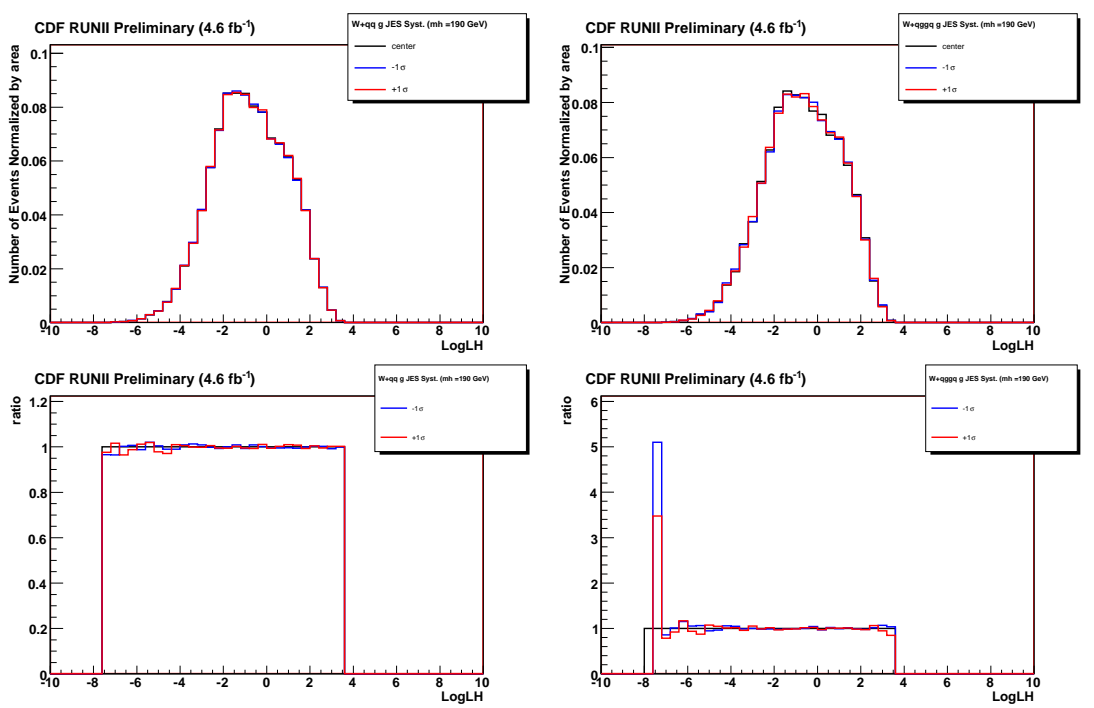

Figure C.31: Likelihood output distribution using the $W+$ jets gluon JES $+1 \sigma$ systematic samples for $m_{H}=190 \mathrm{GeV} / \mathrm{c}^{2}$. The left top plot shows log likelihood output for $W+\mathrm{qq}$ background. The left bottom plot shows ratios which are $\pm 1 \sigma$ /(center value). The right plots show log likelihood output for $W+$ qggq background. 

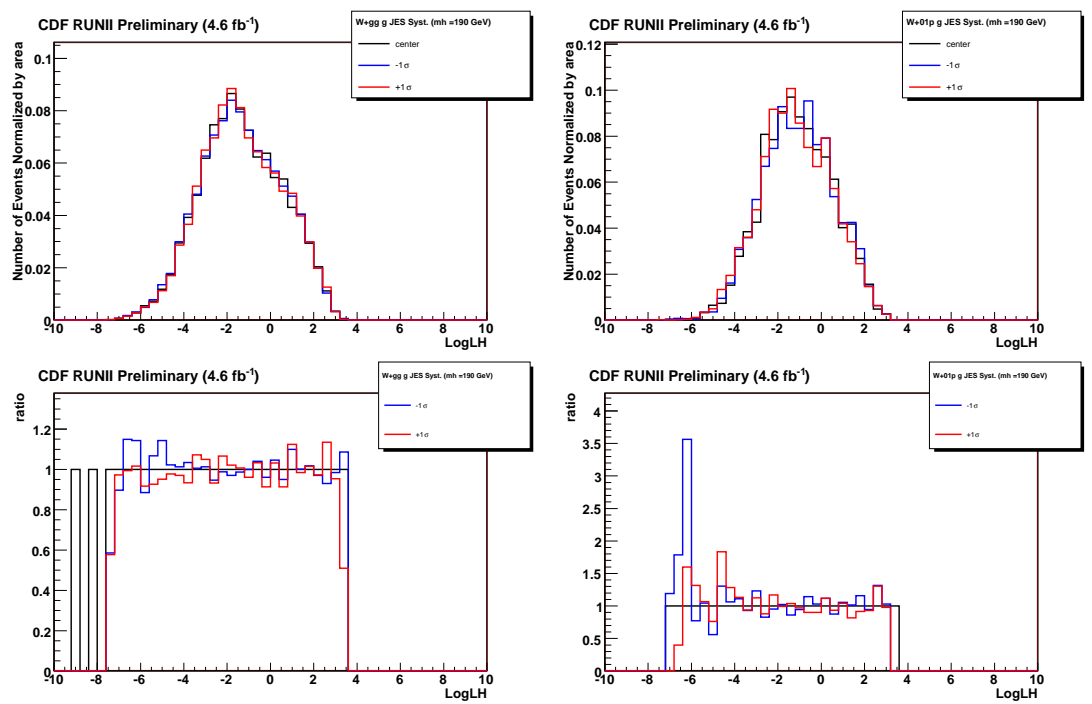

Figure C.32: Likelihood output distribution using the $W+$ jets gluon JES $\pm 1 \sigma$ systematic samples for $m_{H}=190 \mathrm{GeV} / \mathrm{c}^{2}$. The left top plot shows $\log$ likelihood output for $W+\mathrm{gg}$ background. The left bottom plot shows ratios which are $\pm 1 \sigma /$ (center value). The right plot shows log likelihood output for $W+0$ or 1 parton background. 

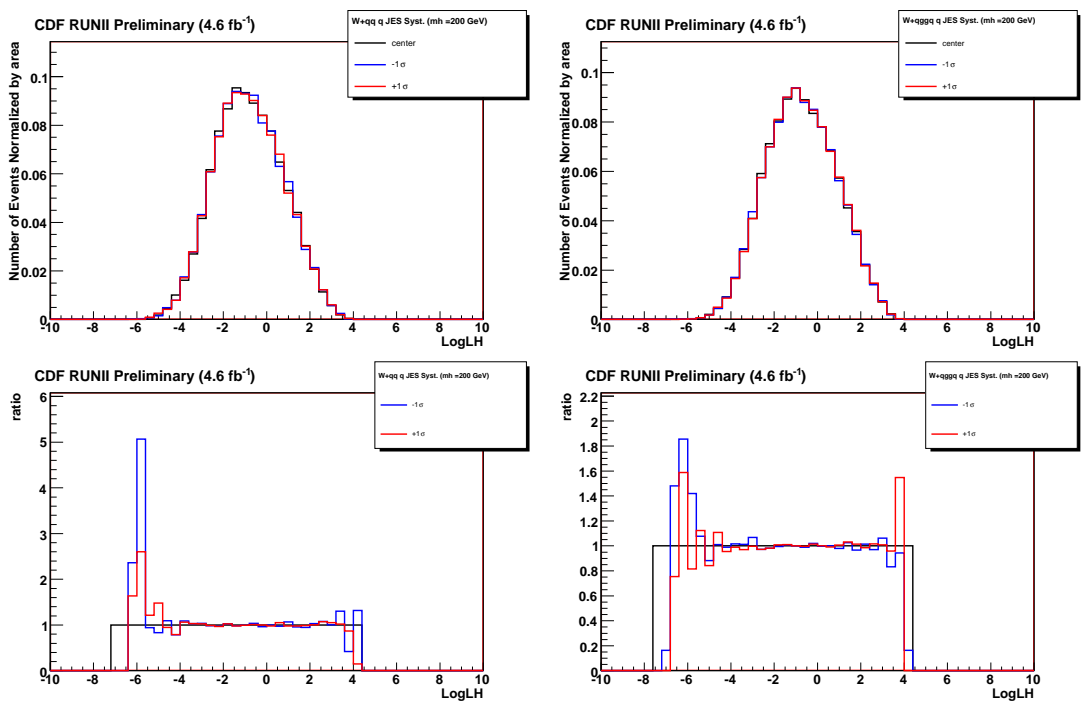

Figure C.33: Likelihood output distribution using the $W+$ jets quark JES $+1 \sigma$ systematic samples for $m_{H}=200 \mathrm{GeV} / \mathrm{c}^{2}$. The left top plot shows log likelihood output for $W+q q$ background. The left bottom plot shows ratios which are $\pm 1 \sigma /$ (center value). The right plots show log likelihood output for $W+$ qggq background. 

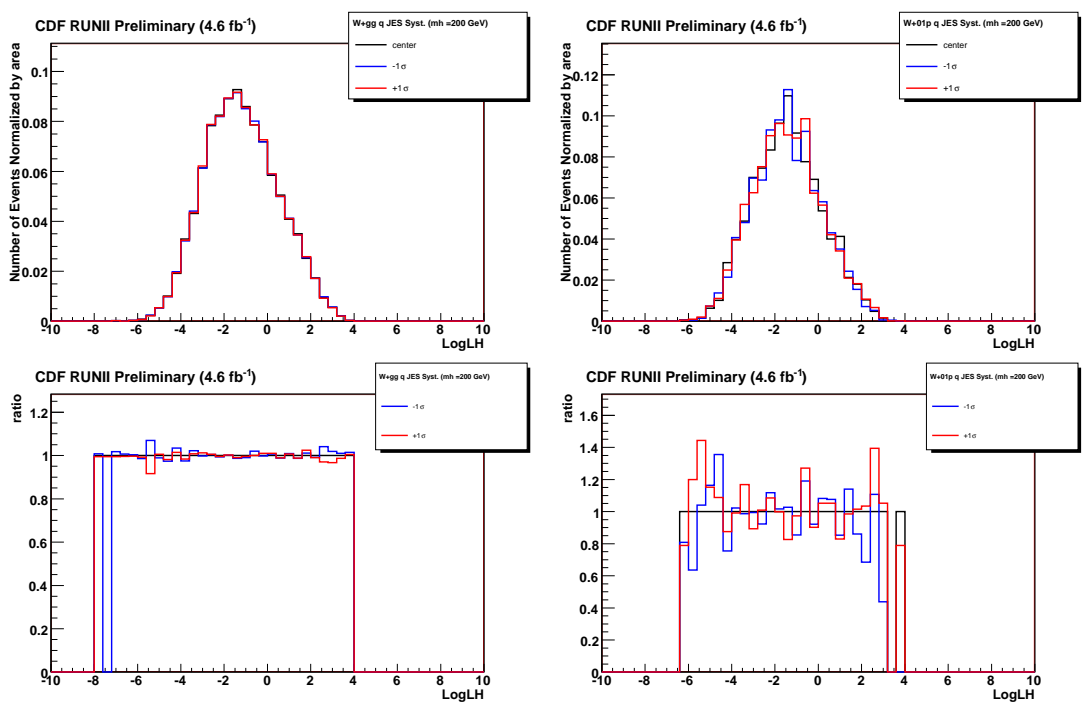

Figure C.34: Likelihood output distribution using the $W+$ jets quark JES $\pm 1 \sigma$ systematic samples for $m_{H}=200 \mathrm{GeV} / \mathrm{c}^{2}$. The left top plot shows log likelihood output for $W+$ gg background. The left bottom plot shows ratios which are $\pm 1 \sigma /$ (center value). The right plots show log likelihood output for $W+0$ or 1 parton background.
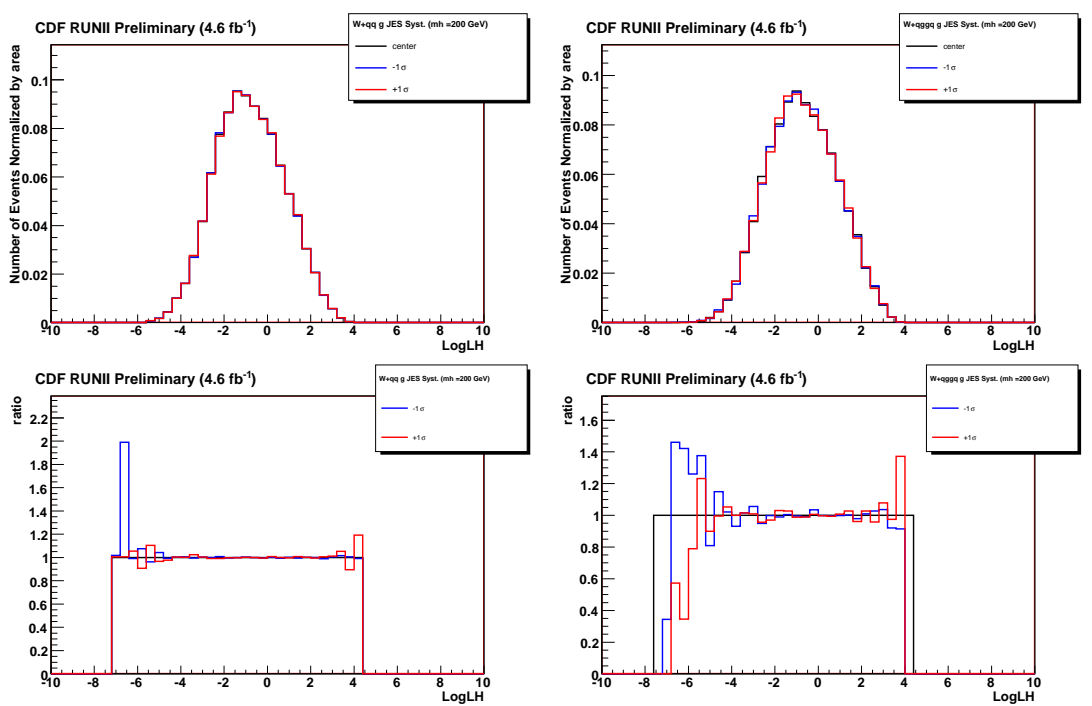

Figure C.35: Likelihood output distribution using the $W+$ jets gluon JES $+1 \sigma$ systematic samples for $m_{H}=200 \mathrm{GeV} / \mathrm{c}^{2}$. The left top plot shows $\log$ likelihood output for $W+\mathrm{qq}$ background. The left bottom plot shows ratios which are $\pm 1 \sigma /($ center value). The right plots show log likelihood output for $W+$ qggq background. 

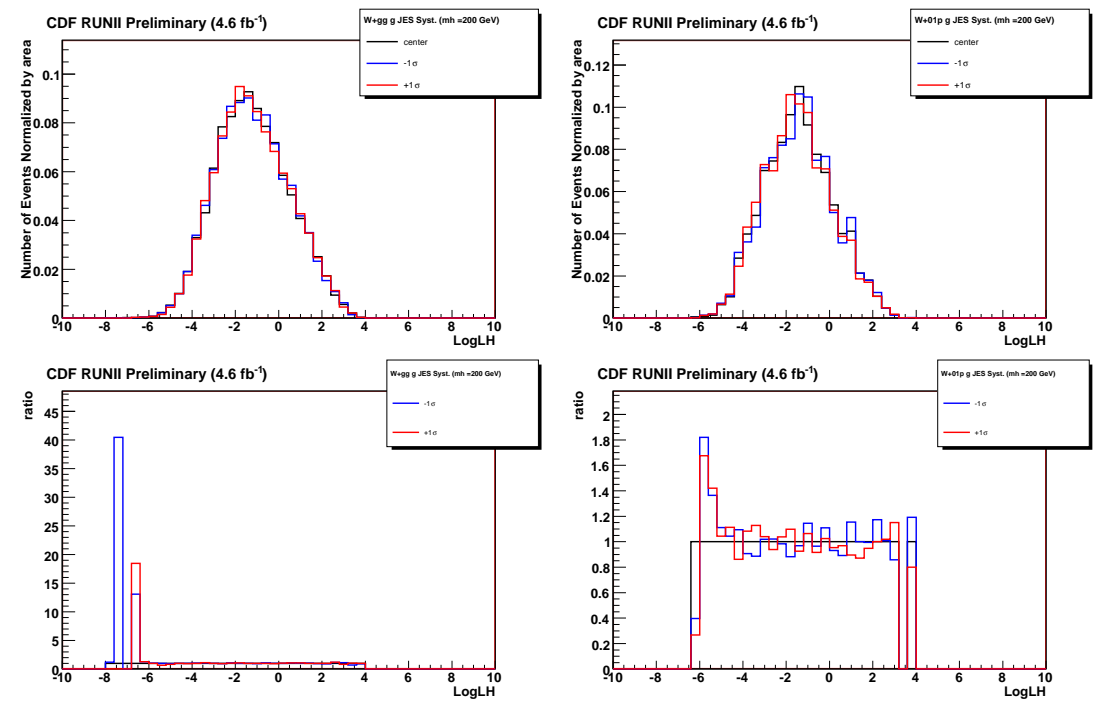

Figure C.36: Likelihood output distribution using the $W+$ jets gluon JES $\pm 1 \sigma$ systematic samples for $m_{H}=200 \mathrm{GeV} / \mathrm{c}^{2}$. The left top plot shows log likelihood output for $W+\mathrm{gg}$ background. The left bottom plot shows ratios which are $\pm 1 \sigma$ /(center value). The right plot shows log likelihood output for $W+0$ or 1 parton background. 


\section{Bibliography}

[1] CDF, T. Aaltonen et al., Search for $H \rightarrow W W^{*}$ Production at CDF Using $8.2 \mathrm{fb}^{-1}$ of Data, CDF Public Note 10599 (2011).

[2] M. Gell-Mann, A Schematic Model of Baryons and Mesons, Phys. Lett. 8, 214 (1964).

[3] G. Altarelli, Partons in Quantum Chromodynamics, Phys. Rept. 81, 1 (1982).

[4] P. W. Higgs, Broken symmetries, massless particles and gauge fields, Phys. Lett. 12, 132 (1964).

[5] P. W. Higgs, BROKEN SYMMETRIES AND THE MASSES OF GAUGE BOSONS, Phys. Rev. Lett. 13, 508 (1964).

[6] S. L. Glashow, Partial Symmetries of Weak Interactions, Nucl. Phys. 22, 579 (1961).

[7] S. Weinberg, A Model of Leptons, Phys. Rev. Lett. 19, 1264 (1967).

[8] A. Salam and J. C. Ward, Electromagnetic and weak interactions, Phys. Lett. 13, 168 (1964).

[9] A. Djouadi, J. Kalinowski, and M. Spira, HDECAY: A program for Higgs boson decays in the standard model and its supersymmetric extension, Comput. Phys. Commun. 108, 56 (1998), arXiv:hep-ph/9704448.

[10] LEP Working Group for Higgs boson searches, R. Barate et al., Search for the standard model Higgs boson at LEP, Phys. Lett. B565, 61 (2003), arXiv:hep-ex/0306033.

[11] CDF Higgs Group, CDF Higgs Page, http://www-cdf.fnal.gov/physics/new/hdg/hdg.html.

[12] Higgs Physics Group at DO, D0 Higgs, http://www-d0.fnal.gov/Run2Physics/higgs/.

[13] The LEP Electroweak Working Group, http://lepewwg.web.cern.ch/LEPEWWG/, .

[14] E. W. J. Cockroft, Experiments with High Velocity Positive Ions. II. The Disintegration of Elements by High Velocity Protons., Proc. of the Royal Soc. of London A137, 229 (1932).

[15] J. Marriner, Stochastic cooling overview, Nucl. Instrum. Meth. A532, 11 (2004), arXiv:physics/0308044.

[16] S. Nagaitsev et al., Experimental demonstration of relativistic electron cooling, Phys. Rev. Lett. 96, 044801 (2006).

[17] On behalf of the CDF, C. S. Hill, Operational experience and performance of the CDFII silicon detector, Nucl. Instrum. Meth. A530, 1 (2004). 
[18] CDF, A. Sill, CDF Run II silicon tracking projects, Nucl. Instrum. Meth. A447, 1 (2000).

[19] CDF, A. A. Affolder et al., Intermediate silicon layers detector for the CDF experiment, Nucl. Instrum. Meth. A453, 84 (2000).

[20] CDF, A. A. Affolder et al., CDF central outer tracker, Nucl. Instrum. Meth. A526, 249 (2004).

[21] CDF-II, D. Acosta et al., A time-of-flight detector in CDF-II, Nucl. Instrum. Meth. A518, 605 (2004).

[22] CDF, L. Balka et al., The CDF Central Electromagnetic Calorimeter, Nucl. Instrum. Meth. A267, 272 (1988).

[23] CDF, M. G. Albrow et al., The CDF plug upgrade electromagnetic calorimeter: Test beam results, Nucl. Instrum. Meth. A480, 524 (2002).

[24] G. Apollinari, K. Goulianos, P. Melese, and M. Lindgren, Shower maximum detector for the CDF plug upgrade calorimeter, Nucl. Instrum. Meth. A412, 515 (1998).

[25] CDF, S. Bertolucci et al., The CDF Central and Endwall Hadron Calorimeter, Nucl. Instrum. Meth. A267, 301 (1988).

[26] G. Ascoli et al., CDF Central Muon Detector, Nucl. Instrum. Meth. A268, 33 (1988).

[27] D. Acosta et al., The performance of the CDF luminosity monitor, Nucl. Instrum. Meth. A494, 57 (2002).

[28] E. J. Thomson et al., Online track processor for the CDF upgrade, IEEE Trans. Nucl. Sci. 49, 1063 (2002).

[29] CDF-II, B. Ashmanskas et al., The CDF silicon vertex trigger, Nucl. Instrum. Meth. A518, 532 (2004), arXiv:physics/0306169.

[30] CDF, D. E. Acosta et al., Measurement of the $t \bar{t}$ production cross section in $p \bar{p}$ collisions at $\sqrt{s}=1.96 \mathrm{TeV}$ using lepton + jets events with secondary vertex $b$-tagging, Phys. Rev. D71, 052003 (2005), arXiv:hep-ex/0410041.

[31] CDF, A. Abulencia et al., Measurements of Inclusive W and Z Cross Sections in p-pbar Collisions at sqrts =1.96 TeV, J. Phys. G34, 2457 (2007), arXiv:hep-ex/0508029.

[32] CDF, F. Abe et al., The Topology of three jet events in $\bar{p} p$ collisions at $\sqrt{s}=1.8 \mathrm{TeV}$, Phys. Rev. D45, 1448 (1992).

[33] A. Bhatti et al., Determination of the jet energy scale at the collider detector at Fermilab, Nucl. Instrum. Meth. A566, 375 (2006), arXiv:hep-ex/0510047.

[34] D. G. et al. (Particle Data Group), Particle Data Group, Eur. Phys. J. C15, 1 (2000).

[35] T. T. W. G. for the CDF and D. Collaborations, Combined CDF and D0 Upper Limits on Standard Model Higgs Boson Production with up to $8.6 \mathrm{fb}^{-1}$ of Data, (2011), arXiv:1107.5518v2.

[36] H. M. Grazzini, , http://theory.fi.infn.it/grazzini/codes.html. 
[37] S. Catani and M. Grazzini, Phys. Rev. Lett. 98 (2007).

[38] M. Grazzini, JHEP 0802, 043 (2008).

[39] A. D. Martin, W. J. Stirling, R. S. Thorne, and G. Watt, , Eur. Phys. J. C63, 189 (2009).

[40] CDF, T. Aaltonen et al., Measurement of Inclusive Jet Cross Sections in $Z / \gamma^{*}+$ jets Production in $p \bar{p}$ Collisions at $\sqrt{s}=1.96 \mathrm{TeV}$, arXiv:0711.3717v1. 